2002

\title{
Mode-I fatigue fracture of interface for fiber -reinforced polymer composite bonded to wood
}

Junhui Jia

West Virginia University

Follow this and additional works at: https://researchrepository.wvu.edu/etd

\section{Recommended Citation}

Jia, Junhui, "Mode-I fatigue fracture of interface for fiber -reinforced polymer composite bonded to wood" (2002). Graduate Theses, Dissertations, and Problem Reports. 2464.

https://researchrepository.wvu.edu/etd/2464 


\title{
Mode-I Fatigue Fracture of Interface for Fiber-Reinforced Polymer Composite Bonded to Wood
}

\author{
Junhui Jia \\ Dissertation submitted to the \\ at West Virginia University \\ in partial fulfillment of the requirements \\ for the Degree of \\ Doctor of Philosophy \\ in \\ Civil Engineering \\ Julio Davalos, Ph.D., Chair \\ Ever Barbero, Ph.D. \\ Elemer Lang, Ph.D. \\ Pizhong Qiao, Ph.D. \\ Indrajit Ray, Ph.D.
} College of Engineering and Mineral Resources

Department of Civil and Environmental Engineering

Morgantown, West Virginia 2002

Keywords: Fatigue, Fracture, Interface, Fiber-Reinforced Polymer (FRP), Wood Copyright 2002 Junhui Jia 


\title{
ABSTRACT \\ Mode-I Fatigue Fracture of Interface for Fiber-Reinforced Polymer Composite Bonded to Wood
}

\author{
Junhui Jia
}

For applications in civil infrastructure rehabilitation and new construction, wood is being reinforced with externally bonded laminates and fabrics of fiber-reinforced polymer (FRP) composites. The potential benefits of external reinforcement with bonded FRP composites have been demonstrated through several studies, and the field implementation of this technology has been successfully proven in numerous projects. However, there is a concern with the long-term performance of the interface bond, since an inadequate bond strength and integrity can render the reinforcement ineffective and lead to premature failure of the structure. The performance of FRP-wood bonded interfaces under static and environmental loads have been explored extensively at WVU, and as an extension of this effort, present study will examine, for the first time, the fatigue behavior of FRP-wood bonded interfaces.

The objective of this study is to develop a combined analytical and experimental fracture mechanics method to evaluate the fatigue behavior of phenolic FRP-red maple wood bonded interfaces under constant cyclic load conditions. The possible effect of loading variables (load ratio, waveform and frequency) on crack propagation rate is studied. The fatigue threshold is determined at the conclusion of this project, and the possible load ratio effect on fatigue threshold is investigated. The study of constant amplitude fatigue on bonded interfaces provides valuable insights into the mechanical processes by which fatigue failure can occur for actual structures or member under varying amplitude cyclic loading.

Fatigue failure of materials and bonded interfaces is a very complex topic, and yet a quite important issue in engineering practices. The application of linear elastic fracture mechanics concepts has resulted in some reasonable and useful explanations of fatigue failure. By modifying the original Paris Law equation, we can efficiently evaluate the fatigue behavior of FRP-wood interface bond-line, and this method can further be used for other similar studies of interface fracture of dissimilar materials. Thus, the findings of this study can eventually lead to the development of guidelines for hybrid material bonded interface design under constant cyclic loading conditions. 


\section{ACKNOWLEDGMENTS}

I would like to express my sincere gratitude and appreciation to Dr. Julio F. Davalos for his guidance and inspiration towards the completion of this research work. His constant support, trust, knowledge, patience and unselfishness have been invaluable to the progress of this work. Thanks are also due to the committee members, Dr. Ever Barbero, Dr. Elemer M. Lang, Dr. Pizhong Qiao and Dr. Indrajit Ray for their time, effort, constructive comments and review of this work.

I am grateful to Dr. Pizhong Qiao, Brent Trimble and David Boyajian for their assistance at the beginning of this research work. Valuable help was provided by laboratory technicians: Dave Turner, Dana Humberson, Paul Frum, and William J. Comstock. Generous help was also offered by wood industry graduate students: Laszlo Bejo and Jody Gray whose collaboration was vital to the completion of my experimental work.

We would like to thank the partial financial support provided by NSF grant CMS9970008, under the program director Dr. Ken P. Chong and the WVU Research Corp. Incentive Grant Program. The adhesive and composite materials were generously donated by INDSPEC Chemical Corp.

Special thanks are due to my parents and my wife for all of their support, advice, and love. My appreciation is also extended to all of my friends and colleagues who are working and used to work in the office of B11, ESB. 


\section{TABLE OF CONTENTS}

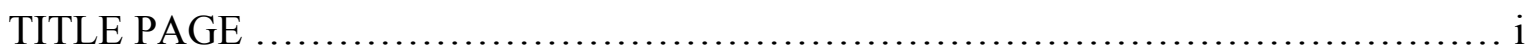

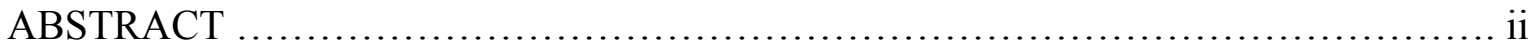

ACKNOWLEDGMENTS ....................................................... iii

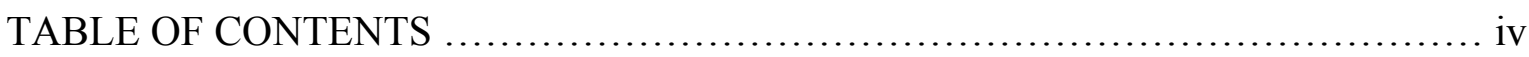

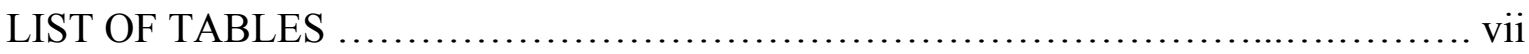

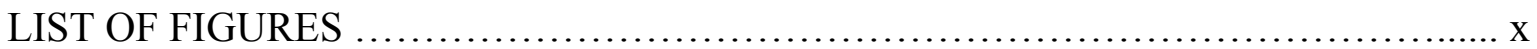

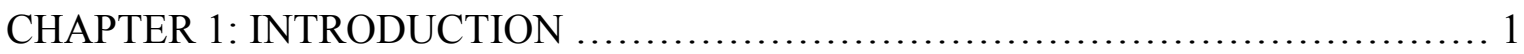

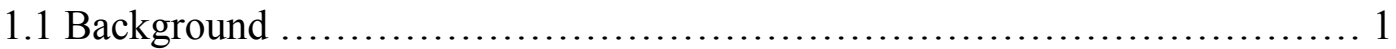

1.2 Objective and Scope ............................................ 3

1.3 Problem Significance ............................................... 5

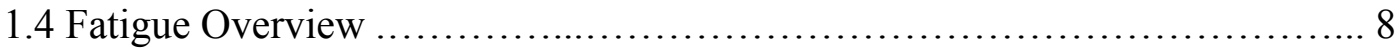

1.4.1 Three stages of fatigue process $\ldots \ldots \ldots \ldots \ldots \ldots \ldots \ldots \ldots \ldots \ldots$

1.4.2 The analysis methods for fatigue problems .................... 13

1.5 The Contoured Double Cantilever Beam (CDCB) Specimen ................ 23

1.5.1 Applications of the CDCB specimens ...................... 27

1.5.2 Previous research at West Virginia University .................. 34

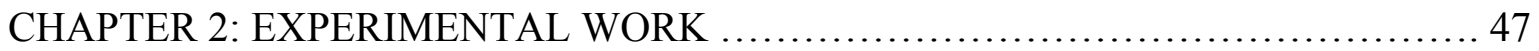

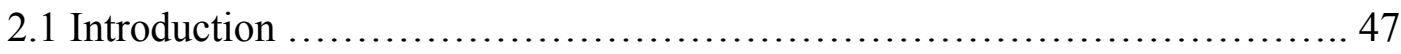

2.2 Materials ........................................................ 50

2.2.1 Red Maple ................................................ 52

2.2.2 Laminated veneer lumber (LVL) ............................. 52

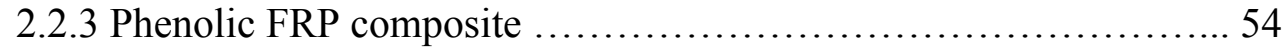

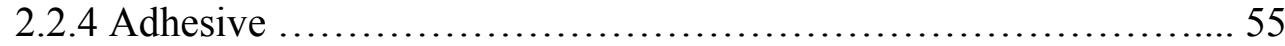

2.3 Mechanical Properties of Materials ...................................... 56

2.4 Shape of the CDCB Specimen .......................................... 57

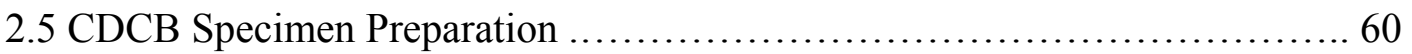


2.6 Fatigue Test

CHAPTER 3: LOAD RATIO EFFECT …........................................ 70

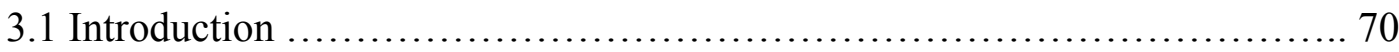

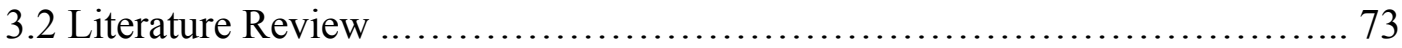

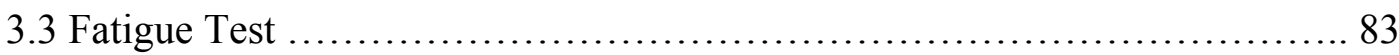

3.3.1 Materials and specimen ....................................... 83

3.3.2 Test procedure .................................................. 84

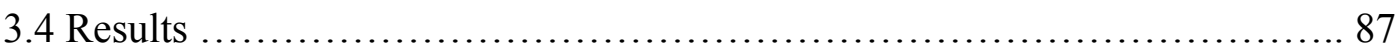

3.4.1 Experimental data processing ............................... 87

3.4.2 Crack growth rate determination by compliance method ........... 114

3.4.3 Constant strain energy release rate range calculation ................ 116

3.4.4 A modified Paris Law model .................................... 117

3.4.5 The crack closure effect ......................................133

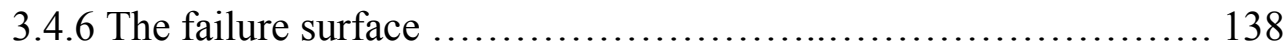

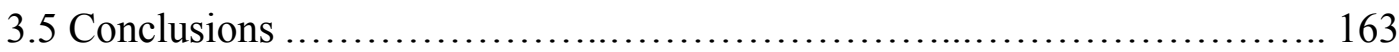

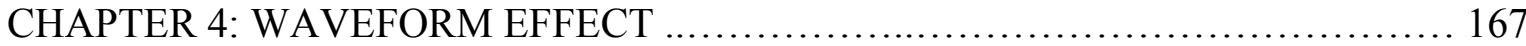

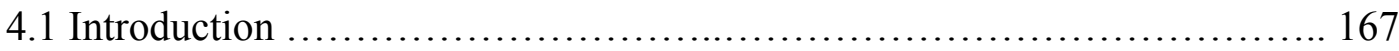

4.2 Literature Review ................................................. 168

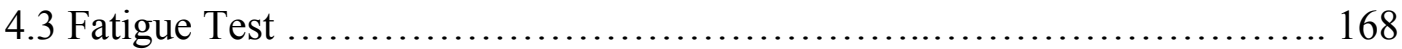

4.3.1 Materials and specimen ......................................... 168

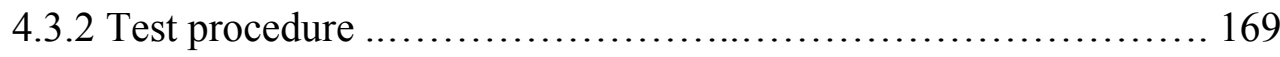

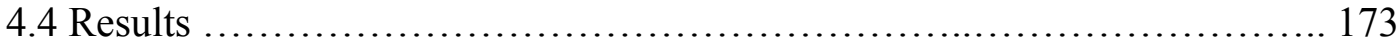

4.4.1 Experimental data processing .................................... 173

4.4.2 Crack growth rate determination by compliance method ............ 195

4.4.3 Constant strain energy release rate range calculation ............... 196

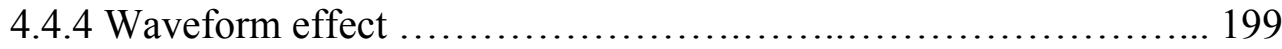

4.4.5 The failure surface …........................................... 205

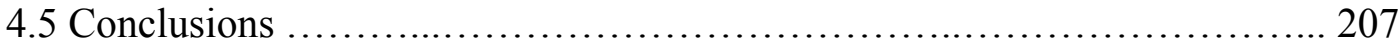




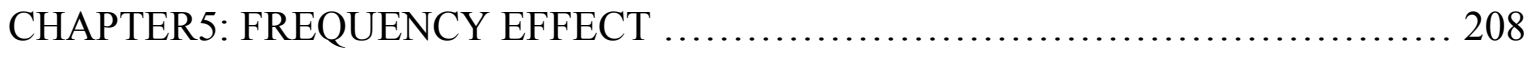

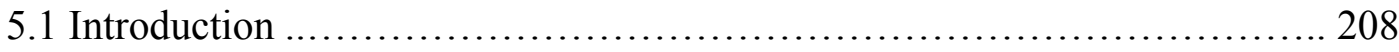

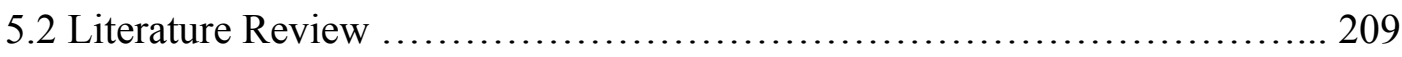

5.3 Fatigue Test ......................................................... 215

5.3.1 Materials and specimen......................................... 215

5.3.2 Test procedure ................................................ 215

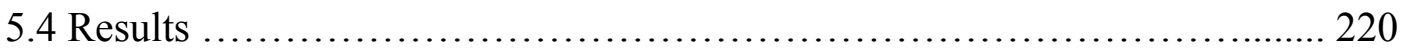

5.4.1 Experimental data processing ............................... 220

5.4.2 Crack growth rate determination by compliance method ............ 234

5.4.3 Constant strain energy release rate range calculation ............... 236

5.4.4 A modified Paris Law model ..................................... 237

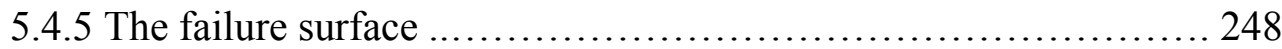

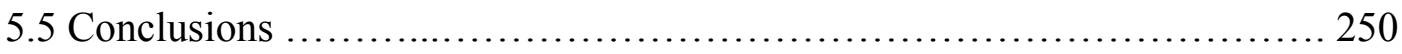

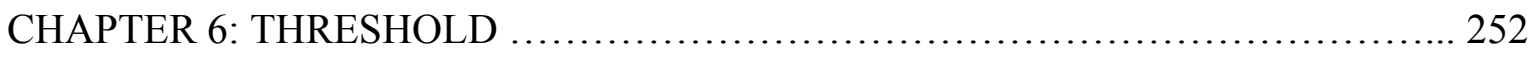

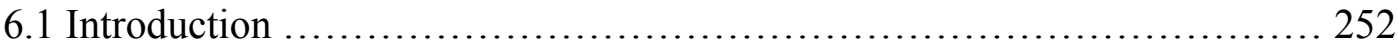

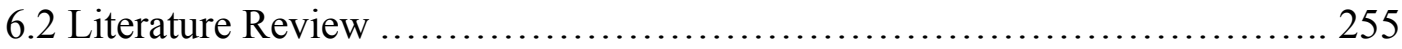

6.3 The Modified Load Shedding Procedure ….............................. 261

6.4 Fatigue Test ....................................................... 264

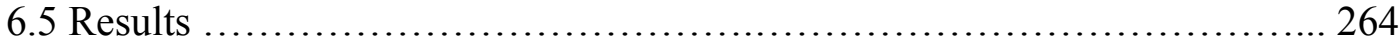

6.5.1 Determination of the fatigue threshold .......................... 264

6.5.2 Load ratio effect on the fatigue threshold ......................... 266

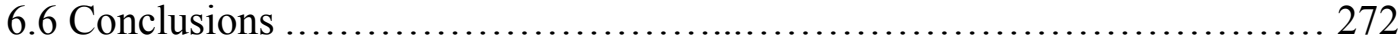

CHAPTER 7: CONCLUSIONS AND RECOMMENDATIONS …................... 275

7.1 Summary and Conclusions .......................................... 276

7.2 Recommendations for Future Work .................................. 282

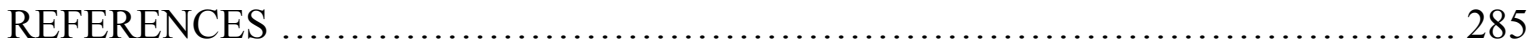




\section{LIST OF TABLES}

Table 1.1 The modified Paris Law equations for fatigue crack propagation 23

Table 2.1 Experimental materials and their sources 51

Table 2.2 Mechanical properties of phenolic FRP composite, laminated veneer lumber (LVL) and red maple wood

Table 3.1 Proposed modified Paris Law equations including load ratio effect 82

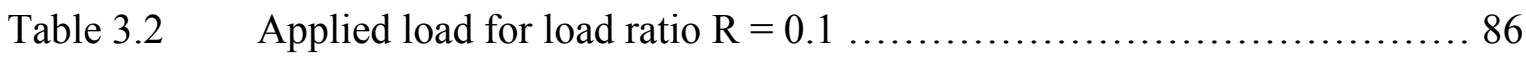

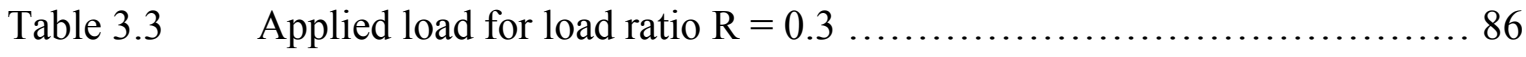

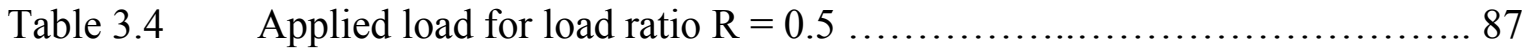

Table 3.5 Experimental results of the COD propagation rate for the load ratio $R=0.1$ under a constant frequency $f=1 \mathrm{~Hz}$ and sinusoidal waveform .......... 113

Table 3.6 Experimental results of the COD propagation rate for the load ratio $R=0.3$ under a constant frequency $f=1 \mathrm{~Hz}$ and sinusoidal waveform ......... 113

Table 3.7 Experimental results of the COD propagation rate for the load ratio $R=0.5$ under a constant frequency $f=1 \mathrm{~Hz}$ and sinusoidal waveform ...........114

Table $3.8 \quad$ The crack propagation rate $d a / d N$ for load ratio $R=0.1 \ldots \ldots \ldots \ldots \ldots . .115$

Table 3.9 The crack propagation rate $d a / d N$ for load ratio $R=0.3 \ldots \ldots \ldots \ldots \ldots . \ldots 115$

Table 3.10 The crack propagation rate $d a / d N$ for load ratio $R=0.5 \ldots \ldots \ldots \ldots \ldots . \ldots 116$

Table 3.11 Material constants $B$ and $m$ in Eq. (3.24) for three load ratios $R \ldots \ldots \ldots 118$

Table 3.12 Material constants $B, m$ and $\lambda$ and coefficient of determination $r^{2}$ for three modified Paris Law equations .................................. 126

Table 3.13 Comparison of the effective or equivalent strain energy release rate range

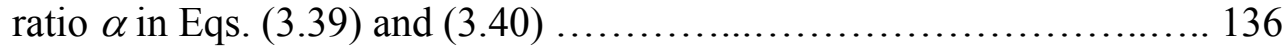




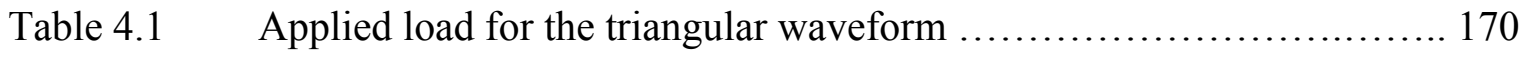

Table 4.2 Applied load for the sinusoidal waveform ......................... 171

Table 4.3 Applied load for the square waveform ........................... 171

Table 4.4 Experimental results of the COD propagation rate for the triangular waveform under the constant load ratio $R=0.5$ and frequency $f=1 \mathrm{~Hz}$

Table 4.5 Experimental results of the COD propagation rate for the sinusoidal waveform under the constant load ratio $R=0.5$ and frequency $f=1 \mathrm{~Hz}$

Table 4.6 Experimental results of the COD propagation rate for the square waveform under the constant load ratio $R=0.5$ and frequency $f=1 \mathrm{~Hz}$ 195

Table 4.7 The crack propagation rate $d a / d N$ for triangular waveform .............. 197

Table 4.8 The crack propagation rate $d a / d N$ for sinusoidal waveform .............. 198

Table 4.9 The crack propagation rate $d a / d N$ for square waveform ................ 198

Table 4.10 Material constants $B$ and $m$ in Eq. (4.6) for three different waveforms under a constant load ratio $R=0.5$ and a frequency $f=1 \mathrm{~Hz}$............... 199

Table 4.11 The crack propagation rate $d a / d N$ for triangular waveform ............. 200

Table 4.12 The crack propagation rate $d a / d N$ for square waveform ................ 200

Table 5.1 Applied load for frequency of $f=0.1 \mathrm{~Hz}$ under constant load ratio $R=0.5$ and sinusoidal waveform ............................................ 217

Table 5.2 Applied load for frequency of $f=1 \mathrm{~Hz}$ under constant load ratio $R=0.5$ and sinusoidal waveform ................................................... 217

Table 5.3 Applied load for frequency of $f=10 \mathrm{~Hz}$ under constant load ratio $R=0.5$

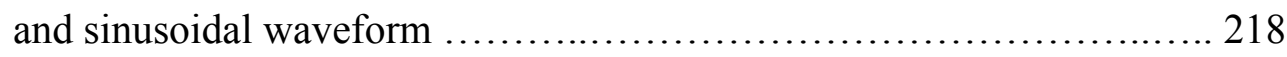

Table 5.4 Experimental results of COD propagation rate for frequency $f=0.1 \mathrm{~Hz}$ under constant load ratio $R=0.5$ and sinusoidal waveform ............. 233

Table 5.5 Experimental results of COD propagation rate for frequency $f=1 \mathrm{~Hz}$ under constant load ratio $R=0.5$ and sinusoidal waveform $\ldots \ldots \ldots \ldots \ldots \ldots . \ldots 233$ 
Table 5.6 Experimental results of COD propagation rate for frequency $f=10 \mathrm{~Hz}$ under constant load ratio $R=0.5$ and sinusoidal waveform .............. 234

Table 5.7 The crack propagation rate $d a / d N$ for frequency $f=0.1 \mathrm{~Hz} \ldots \ldots \ldots \ldots \ldots . .235$

Table 5.8 The crack propagation rate $d a / d N$ for frequency $f=1 \mathrm{~Hz} \ldots \ldots \ldots \ldots \ldots . . .235$

Table 5.9 The crack propagation rate $d a / d N$ for frequency $f=10 \mathrm{~Hz} \ldots \ldots \ldots \ldots \ldots . .236$

Table 5.10 Material constants $B$ and $m$ in Eq. (5.14) for three different frequencies $f$ 238

Table 5.11 The crack propagation rate $d a / d N$ for $f=0.1 \mathrm{~Hz} \ldots \ldots \ldots \ldots \ldots \ldots \ldots \ldots \ldots . \ldots \ldots \ldots \ldots$

Table 5.12 The crack propagation rate $d a / d N$ for $f=10 \mathrm{~Hz} \ldots \ldots \ldots \ldots \ldots \ldots \ldots \ldots \ldots . \ldots \ldots . \ldots \ldots$

Table 6.1 Experimental results of strain energy rate parameters at the fatigue threshold 265 


\section{LIST OF FIGURES}

Figure 1.1 A schematic illustration of three stages of a fatigue process (Suresh 1998)

Figure 1.2 An approximate representation of the fatigue process by Dowling et al. (2000) 11

Figure 1.3 A demonstration of the envelope area for the area method 29

Figure 1.4 The geometry of one half of a CDCB specimen from Madabhusi-Raman (1995) 35

Figure 1.5 The transformed cross section of a CDCB specimen 41

Figure 2.1 Modes of loading that can be applied to a crack (From Anderson 1994) .. 48

Figure 2.2 Lay-up of the yellow poplar laminated veneer lumber (LVL) ............. 53

Figure 2.3 Material architecture of the phenolic FRP strip ........................ 54

Figure 2.4 Geometry of the CDCB specimen for fatigue tests ..................... 58

Figure 2.5 Side view of the CDCB specimen assembly under pressure for curing the

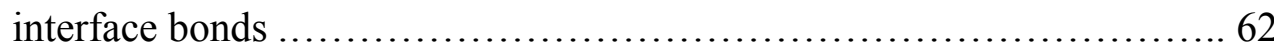

Figure 2.6 Front view of the CDCB specimen assembly under pressure for curing the

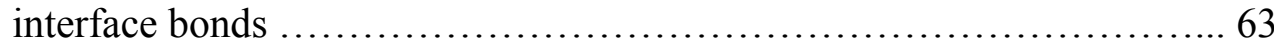

Figure 2.7 A schematic illustration of constant amplitude cyclic loading ............ 66

Figure 2.8 The MTS servo hydraulic testing machine for Mode-I fatigue tests ........ 67

Figure 2.9 Experimental setup for mode-I fatigue tests ......................... 68

Figure 2.10 Photograph of a specimen under fatigue tests .............................. 69

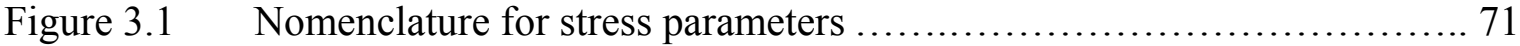

Figure 3.2 A schematic illustration of cyclic loading at different load ratios ......... 85

Figure 3.3 A typical diagram of COD versus number of cycles for a CDCB specimen 
Figure 3.4 COD versus number of cycles for a CDCB specimen under 8-80 lb. cyclic loading with a frequency of $1 \mathrm{~Hz}$, load ratio $R=0.1$ and sinusoidal waveform 90

Figure 3.5 COD versus number of cycles for a CDCB specimen under 8.5-85 lb. cyclic loading with a frequency of $1 \mathrm{~Hz}$, load ratio $R=0.1$ and sinusoidal waveform 91

Figure 3.6 COD versus number of cycles for a CDCB specimen under 9-90 lb. cyclic loading with a frequency of $1 \mathrm{~Hz}$, load ratio $R=0.1$ and sinusoidal waveform

Figure 3.7 COD versus number of cycles for a CDCB specimen under 10-100 lb. cyclic loading with a frequency of $1 \mathrm{~Hz}$, load ratio $R=0.1$ and sinusoidal waveform 93

Figure 3.8 COD versus number of cycles for a CDCB specimen under 11-110 lb. cyclic loading with a frequency of $1 \mathrm{~Hz}$, load ratio $R=0.1$ and sinusoidal waveform

Figure 3.9 COD versus number of cycles for a CDCB specimen under 12-120 lb. cyclic loading with a frequency of $1 \mathrm{~Hz}$, load ratio $R=0.1$ and sinusoidal waveform 95

Figure 3.10 COD versus number of cycles for a CDCB specimen under 13-130 lb. cyclic loading with a frequency of $1 \mathrm{~Hz}$, load ratio $R=0.1$ and sinusoidal waveform 96

Figure 3.11 COD versus number of cycles for a CDCB specimen under 24-80 lb. cyclic loading with a frequency of $1 \mathrm{~Hz}$, load ratio $R=0.3$ and sinusoidal waveform 97

Figure 3.12 COD versus number of cycles for a CDCB specimen under 24.5-85 lb. cyclic loading with a frequency of $1 \mathrm{~Hz}$, load ratio $R=0.3$ and sinusoidal waveform 98

Figure 3.13 COD versus number of cycles for a CDCB specimen under 27-90 lb. cyclic loading with a frequency of $1 \mathrm{~Hz}$, load ratio $R=0.3$ and sinusoidal waveform 99 
Figure 3.14 COD versus number of cycles for a CDCB specimen under 30-100 lb. cyclic loading with a frequency of $1 \mathrm{~Hz}$, load ratio $R=0.3$ and sinusoidal waveform

Figure 3.15 COD versus number of cycles for a CDCB specimen under 33-110 lb. cyclic loading with a frequency of $1 \mathrm{~Hz}$, load ratio $R=0.3$ and sinusoidal waveform 101

Figure 3.16 COD versus number of cycles for a CDCB specimen under 36-120 lb. cyclic loading with a frequency of $1 \mathrm{~Hz}$, load ratio $R=0.3$ and sinusoidal waveform

Figure 3.17 COD versus number of cycles for a CDCB specimen under 37.5-125 lb. cyclic loading with a frequency of $1 \mathrm{~Hz}$, load ratio $\mathrm{R}=0.3$ and sinusoidal waveform 103

Figure 3.18 COD versus number of cycles for a CDCB specimen under 39-130 lb. cyclic loading with a frequency of $1 \mathrm{~Hz}$, load ratio $R=0.3$ and sinusoidal waveform 104

Figure 3.19 COD versus number of cycles for a CDCB specimen under 40-80 lb. cyclic loading with a frequency of $1 \mathrm{~Hz}$, load ratio $R=0.5$ and sinusoidal waveform 105

Figure 3.20 COD versus number of cycles for a CDCB specimen under 42.5-85 lb. cyclic loading with a frequency of $1 \mathrm{~Hz}$, load ratio $R=0.5$ and sinusoidal waveform 106

Figure 3.21 COD versus number of cycles for a CDCB specimen under 45-90 lb. cyclic loading with a frequency of $1 \mathrm{~Hz}$, load ratio $R=0.5$ and sinusoidal waveform 107

Figure 3.22 COD versus number of cycles for a CDCB specimen under 47.5-95 lb. cyclic loading with a frequency of $1 \mathrm{~Hz}$, load ratio $R=0.5$ and sinusoidal waveform 108

Figure 3.23 COD versus number of cycles for a CDCB specimen under 50-100 lb. cyclic loading with a frequency of $1 \mathrm{~Hz}$, load ratio $R=0.5$ and sinusoidal waveform 109 
Figure 3.24 COD versus number of cycles for a CDCB specimen under 55-110 lb. cyclic loading with a frequency of $1 \mathrm{~Hz}$, load ratio $R=0.5$ and sinusoidal waveform

Figure 3.25 COD versus number of cycles for a CDCB specimen under 60-120 lb. cyclic loading with a frequency of $1 \mathrm{~Hz}$, load ratio $R=0.5$ and sinusoidal waveform

Figure 3.26 COD versus number of cycles for a CDCB specimen under 65-130 lb. cyclic loading with a frequency of $1 \mathrm{~Hz}$, load ratio $R=0.5$ and sinusoidal waveform

Figure 3.27 The log-log plot of crack propagation rate $d a / d N$ versus the strain energy release rate range $\Delta G$ for load ratio $\mathrm{R}=0.1$

Figure 3.28 The log-log plot of crack propagation rate $d a / d N$ versus the strain energy release rate range $\Delta G$ for load ratio $\mathrm{R}=0.3$

Figure 3.29 The log-log plot of crack propagation rate $d a / d N$ versus the strain energy release rate range $\Delta G$ for load ratio $\mathrm{R}=0.5$

Figure 3.30 The log-log plot of crack propagation rate $d a / d N$ versus the strain energy release rate range $\Delta G$ for different load ratios 122

Figure 3.31 The log-log plot of crack propagation rate $d a / d N$ versus the strain energy release rate range $2 \Delta G G_{\text {mean }}$ for different load ratios

Figure 3.32 The log-log plot of crack propagation rate $d a / d N$ versus the strain energy release rate range $G_{\min }$ for different load ratios 128

Figure 3.33 The log-log plot of crack propagation rate $d a / d N$ versus the strain energy release rate range $G_{\text {mean }}$ for different load ratios 129

Figure 3.34 The log-log plot of crack propagation rate $d a / d N$ versus the strain energy release rate range $\Delta G /(1-R)^{1-\lambda}$ for different load ratios 130

Figure 3.35 The log-log plot of crack propagation rate $d a / d N$ versus the strain energy release rate range $\Delta G^{(1-\lambda)} G^{\lambda}{ }_{\min }$ for different load ratios

Figure 3.36 The $\log -\log$ plot of crack propagation rate $d a / d N$ versus the strain energy release rate range $\Delta G^{(1-\lambda)} G_{\text {mean }}^{\lambda}$ for different load ratios

Figure 3.37 Fractured faces for a CDCB specimen under 8-80 lb. cyclic loading with a frequency $f=1 \mathrm{~Hz}$, load ratio $R=0.1$ and sinusoidal waveform 139 
Figure 3.38 Fractured faces for a CDCB specimen under 8.5-85 lb. cyclic loading with a frequency $f=1 \mathrm{~Hz}$, load ratio $R=0.1$ and sinusoidal waveform ........ 140

Figure 3.39 Fractured faces for a CDCB specimen under 9-90 lb. cyclic loading with a frequency $f=1 \mathrm{~Hz}$, load ratio $R=0.1$ and sinusoidal waveform ........ 141

Figure 3.40 Fractured faces for a CDCB specimen under 10-100 lb. cyclic loading with a frequency $f=1 \mathrm{~Hz}$, load ratio $R=0.1$ and sinusoidal waveform ....... 142

Figure 3.41 Fractured faces for a CDCB specimen under 11-110 lb. cyclic loading with a frequency $f=1 \mathrm{~Hz}$, load ratio $R=0.1$ and sinusoidal waveform ....... 143

Figure 3.42 Fractured faces for a CDCB specimen under 12-120 lb. cyclic loading with a frequency $f=1 \mathrm{~Hz}$, load ratio $R=0.1$ and sinusoidal waveform ....... 144

Figure 3.43 Fractured faces for a CDCB specimen under 13-130 lb. cyclic loading with a frequency $f=1 \mathrm{~Hz}$, load ratio $R=0.1$ and sinusoidal waveform ....... 145

Figure 3.44 Fractured faces for a CDCB specimen under 24-80 lb. cyclic loading with a frequency $f=1 \mathrm{~Hz}$, load ratio $R=0.3$ and sinusoidal waveform ........ 146

Figure 3.45 Fractured faces for a CDCB specimen under 25.5-85 lb. cyclic loading with a frequency $f=1 \mathrm{~Hz}$, load ratio $R=0.3$ and sinusoidal waveform ...... 147

Figure 3.46 Fractured faces for a CDCB specimen under 27-90 lb. cyclic loading with a frequency $f=1 \mathrm{~Hz}$, load ratio $R=0.3$ and sinusoidal waveform ........ 148

Figure 3.47 Fractured faces for a CDCB specimen under 30-100 lb. cyclic loading with a frequency $f=1 \mathrm{~Hz}$, load ratio $R=0.3$ and sinusoidal waveform ....... 149

Figure 3.48 Fractured faces for a CDCB specimen under 33-110 lb. cyclic loading with a frequency $f=1 \mathrm{~Hz}$, load ratio $R=0.3$ and sinusoidal waveform ....... 150

Figure 3.49 Fractured faces for a CDCB specimen under 36-120 lb. cyclic loading with a frequency $f=1 \mathrm{~Hz}$, load ratio $R=0.3$ and sinusoidal waveform ....... 151

Figure 3.50 Fractured faces for a CDCB specimen under 37.5-125 lb. cyclic loading with a frequency $f=1 \mathrm{~Hz}$, load ratio $R=0.3$ and sinusoidal waveform 152

Figure 3.51 Fractured faces for a CDCB specimen under 39-130 lb. cyclic loading with a frequency $f=1 \mathrm{~Hz}$, load ratio $R=0.3$ and sinusoidal waveform ....... 153

Figure 3.52 Fractured faces for a CDCB specimen under 40-80 lb. cyclic loading with a frequency $f=1 \mathrm{~Hz}$, load ratio $R=0.5$ and sinusoidal waveform 154 
Figure 3.53 Fractured faces for a CDCB specimen under 42.5-85 lb. cyclic loading with a frequency $f=1 \mathrm{~Hz}$, load ratio $R=0.5$ and sinusoidal waveform ...... 155

Figure 3.54 Fractured faces for a CDCB specimen under 45-90 lb. cyclic loading with a frequency $f=1 \mathrm{~Hz}$, load ratio $R=0.5$ and sinusoidal waveform ........ 156

Figure 3.55 Fractured faces for a CDCB specimen under 47.5-95 lb. cyclic loading with a frequency $f=1 \mathrm{~Hz}$, load ratio $R=0.5$ and sinusoidal waveform $\ldots \ldots . .157$

Figure 3.56 Fractured faces for a CDCB specimen under 50-100 lb. cyclic loading with a frequency $f=1 \mathrm{~Hz}$, load ratio $R=0.5$ and sinusoidal waveform ....... 158

Figure 3.57 Fractured faces for a CDCB specimen under 55-110 lb. cyclic loading with a frequency $f=1 \mathrm{~Hz}$, load ratio $R=0.5$ and sinusoidal waveform ...... 159

Figure 3.58 Fractured faces for a CDCB specimen under 60-120 lb. cyclic loading with a frequency $f=1 \mathrm{~Hz}$, load ratio $R=0.5$ and sinusoidal waveform ...... 160

Figure 3.59 Fractured faces for a CDCB specimen under 65-130 lb. cyclic loading with a frequency $f=1 \mathrm{~Hz}$, load ratio $R=0.5$ and sinusoidal waveform ....... 161

Figure 3.60 Fractured faces having fiber bridging for a CDCB specimen under 65-130 lb. cyclic loading with a frequency $f=1 \mathrm{~Hz}$, load ratio $R=0.5$ and sinusoidal waveform 162

Figure 4.1 A schematic illustration of cyclic loading for different waveform ........ 172 Figure 4.2 COD versus number of cycles for a CDCB specimen under 40-80 lb. cyclic loading with a frequency $f=1 \mathrm{~Hz}$, load ratio $R=0.5$ and triangular waveform 174

Figure 4.3 COD versus number of cycles for a CDCB specimen under 45-90 lb. cyclic loading with a frequency $f=1 \mathrm{~Hz}$, load ratio $R=0.5$ and triangular waveform 175

Figure 4.4 COD versus number of cycles for a CDCB specimen under 50-100 lb. cyclic loading with a frequency $f=1 \mathrm{~Hz}$, load ratio $R=0.5$ and triangular waveform 176

Figure 4.5 COD versus number of cycles for a CDCB specimen under 55-110 lb. cyclic loading with a frequency $f=1 \mathrm{~Hz}$, load ratio $R=0.5$ and triangular waveform 
Figure 4.6 COD versus number of cycles for a CDCB specimen under 60-120 lb. cyclic loading with a frequency $f=1 \mathrm{~Hz}$, load ratio $R=0.5$ and triangular waveform

Figure 4.7 COD versus number of cycles for a CDCB specimen under 65-130 lb. cyclic loading with a frequency $f=1 \mathrm{~Hz}$, load ratio $R=0.5$ and triangular waveform

Figure 4.8 COD versus number of cycles for a CDCB specimen under 40-80 lb. cyclic loading with a frequency $f=1 \mathrm{~Hz}$, load ratio $R=0.5$ and sinusoidal waveform

Figure 4.9 COD versus number of cycles for a CDCB specimen under 42.5-85 lb. cyclic loading with a frequency $f=1 \mathrm{~Hz}$, load ratio $R=0.5$ and sinusoidal waveform

Figure 4.10 COD versus number of cycles for a CDCB specimen under 45-90 lb. cyclic loading with a frequency $f=1 \mathrm{~Hz}$, load ratio $R=0.5$ and sinusoidal waveform 182

Figure 4.11 COD versus number of cycles for a CDCB specimen under 47.5-95 lb. cyclic loading with a frequency $f=1 \mathrm{~Hz}$, load ratio $R=0.5$ and sinusoidal waveform 183

Figure 4.12 COD versus number of cycles for a CDCB specimen under 50-100 lb. cyclic loading with a frequency $f=1 \mathrm{~Hz}$, load ratio $R=0.5$ and sinusoidal waveform 184

Figure 4.13 COD versus number of cycles for a CDCB specimen under 55-110 lb. cyclic loading with a frequency $f=1 \mathrm{~Hz}$, load ratio $R=0.5$ and sinusoidal waveform 185

Figure 4.14 COD versus number of cycles for a CDCB specimen under 60-120 lb. cyclic loading with a frequency $f=1 \mathrm{~Hz}$, load ratio $R=0.5$ and sinusoidal waveform 186

Figure 4.15 COD versus number of cycles for a CDCB specimen under 65-130 lb. cyclic loading with a frequency $f=1 \mathrm{~Hz}$, load ratio $R=0.5$ and sinusoidal waveform 187 
Figure 4.16 COD versus number of cycles for a CDCB specimen under 40-80 lb. cyclic loading with a frequency $f=1 \mathrm{~Hz}$, load ratio $R=0.5$ and square waveform

Figure 4.17 COD versus number of cycles for a CDCB specimen under 45-90 lb. cyclic loading with a frequency $f=1 \mathrm{~Hz}$, load ratio $R=0.5$ and square waveform

Figure 4.18 COD versus number of cycles for a CDCB specimen under 50-100 lb. cyclic loading with a frequency $f=1 \mathrm{~Hz}$, load ratio $R=0.5$ and square waveform

Figure 4.19 COD versus number of cycles for a CDCB specimen under 55-110 lb. cyclic loading with a frequency $f=1 \mathrm{~Hz}$, load ratio $R=0.5$ and square waveform

Figure 4.20 COD versus number of cycles for a CDCB specimen under 60-120 lb. cyclic loading with a frequency $f=1 \mathrm{~Hz}$, load ratio $R=0.5$ and square waveform

Figure 4.21 COD versus number of cycles for a CDCB specimen under 65-130 lb. cyclic loading with a frequency $f=1 \mathrm{~Hz}$, load ratio $R=0.5$ and square waveform

Figure 4.22 The log-log plot of the crack propagation rate $d a / d N$ versus the strain energy release rate range $\Delta G$ for the triangular waveform under a constant load ratio $R=0.5$ and a frequency $f=1 \mathrm{~Hz}$ 201

Figure 4.23 The log-log plot of the crack propagation rate $d a / d N$ versus the strain energy release rate range $\Delta G$ for the sinusoidal waveform under a constant load ratio $R=0.5$ and a frequency $f=1 \mathrm{~Hz}$ 202

Figure 4.24 The log-log plot of the crack propagation rate $d a / d N$ versus the strain energy release rate range $\Delta G$ for the square waveform under a constant load ratio $R=0.5$ and a frequency $f=1 \mathrm{~Hz}$ 203

Figure 4.25 The log-log plot of the crack propagation rate $d a / d N$ versus the strain energy release rate range $\Delta G$ for different waveforms under a constant load

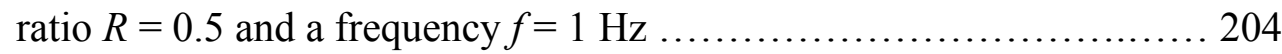


Figure 4.26 A typical fracture surface for the phenolic FRP-red maple wood CDCB specimen under different waveforms .................................. 206

Figure 5.1 A schematic illustration of cyclic loading at different frequencies ....... 219 Figure 5.2 COD versus number of cycles for a CDCB specimen under 40-80 lb. cyclic loading with a frequency $f=0.1 \mathrm{~Hz}$, load ratio $R=0.5$ and sinusoidal

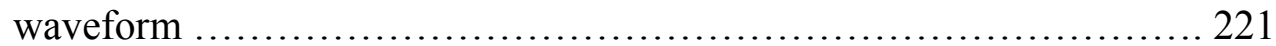

Figure 5.3 COD versus number of cycles for a CDCB specimen under 45-90 lb. cyclic loading with a frequency $f=0.1 \mathrm{~Hz}$, load ratio $R=0.5$ and sinusoidal

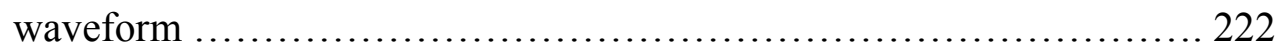

Figure 5.4 COD versus number of cycles for a CDCB specimen under 50-100 lb. cyclic loading with a frequency $f=0.1 \mathrm{~Hz}$, load ratio $R=0.5$ and sinusoidal

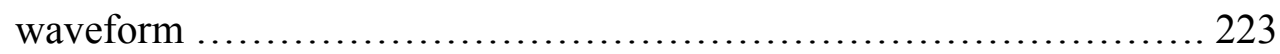

Figure 5.5 COD versus number of cycles for a CDCB specimen under 55-110 lb. cyclic loading with a frequency $f=0.1 \mathrm{~Hz}$, load ratio $R=0.5$ and sinusoidal

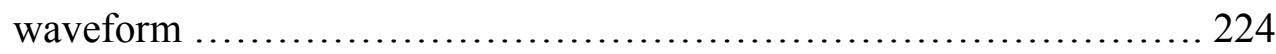

Figure 5.6 COD versus number of cycles for a CDCB specimen under 60-120 lb. cyclic loading with a frequency $f=0.1 \mathrm{~Hz}$, load ratio $R=0.5$ and sinusoidal

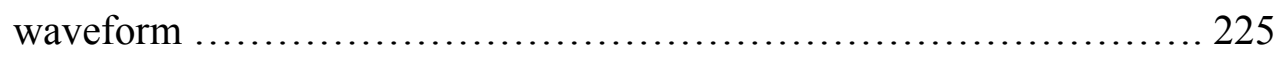

Figure 5.7 COD versus number of cycles for a CDCB specimen under 65-130 lb. cyclic loading with a frequency $f=0.1 \mathrm{~Hz}$, load ratio $R=0.5$ and sinusoidal waveform ....................................................... 226

Figure 5.8 COD versus number of cycles for a CDCB specimen under 40-80 lb. cyclic loading with a frequency $f=10 \mathrm{~Hz}$, load ratio $R=0.5$ and sinusoidal

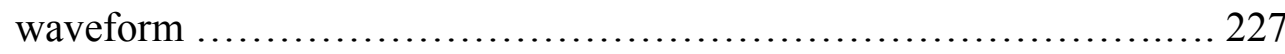

Figure 5.9 COD versus number of cycles for a CDCB specimen under 45-90 lb. cyclic loading with a frequency $\mathrm{f}=10 \mathrm{~Hz}$, load ratio $\mathrm{R}=0.5$ and sinusoidal

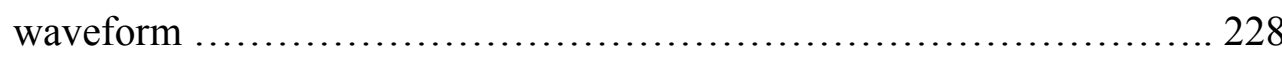


Figure 5.10 COD versus number of cycles for a CDCB specimen under 50-100 lb. cyclic loading with a frequency $f=10 \mathrm{~Hz}$, load ratio $R=0.5$ and sinusoidal

Figure 5.11 COD versus number of cycles for a CDCB specimen under 55-110 lb. cyclic loading with a frequency $f=10 \mathrm{~Hz}$, load ratio $R=0.5$ and sinusoidal

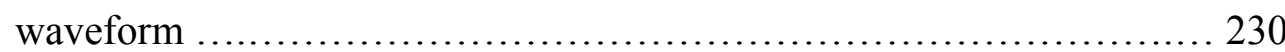

Figure 5.12 COD versus number of cycles for a CDCB specimen under 60-120 lb. cyclic loading with a frequency $f=10 \mathrm{~Hz}$, load ratio $R=0.5$ and sinusoidal 231

Figure 5.13 COD versus number of cycles for a CDCB specimen under 65-130 lb. cyclic loading with a frequency $f=10 \mathrm{~Hz}$, load ratio $R=0.5$ and sinusoidal 232

Figure 5.14 The log-log plot of crack propagation rate $d a / d N$ versus the strain energy release rate range $\Delta G$ for frequency $f=0.1 \mathrm{~Hz}$ 239

Figure 5.15 The log-log plot of crack propagation rate $d a / d N$ versus the strain energy release rate range $\Delta G$ for frequency $f=1.0 \mathrm{~Hz}$

Figure 5.16 The log-log plot of crack propagation rate $d a / d N$ versus the strain energy release rate range $\Delta G$ for frequency $f=10 \mathrm{~Hz} \ldots \ldots \ldots \ldots \ldots \ldots \ldots \ldots . \ldots 24$

Figure 5.17 The log-log plot of crack propagation rate $d a / d N$ versus the strain energy release rate range $\Delta G$ for the frequencies of $0.1,1.0$ and $10 \mathrm{~Hz} \ldots \ldots \ldots .244$

Figure 5.18 The log-log plot of crack propagation rate $d t / d N$ versus the strain energy release rate range $\Delta G$ for the frequencies of $0.1,1.0$ and $10 \mathrm{~Hz} \ldots \ldots \ldots .245$

Figure 5.19 The log-log plot of the crack propagation rate $d a / d N$ versus $\Delta G^{0.07} G_{\text {mean }} 0.93$

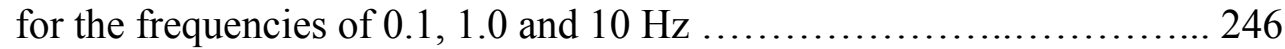

Figure 5.20 The log-log plot of the crack propagation rate $d a / d N$ versus $f$ ${ }^{0.05} \Delta G^{0.07} G_{\text {mean }}{ }^{0.93}$ for the frequencies of $0.1,1.0$ and $10 \mathrm{~Hz}$

Figure 5.21 A typical fracture surface for the phenolic FRP-red maple wood CDCB specimen under different frequencies

Figure 6.1 A typical $G$ decreasing test by stepped load shedding 263 
Figure 6.2 A schematic illustration of Schmidt and Paris's model (1973), where $\mathrm{K}_{\mathrm{cl}}$ and $\mathrm{K}_{\mathrm{c}}$ are the stress intensities at closure and at final failure, respectively, and $\mathrm{R}_{\mathrm{cl}}$ is the critical load ratio above which crack closure effects are minimal

Figure 6.3 Effect of load ratio $R$ on the fatigue threshold $\Delta G_{t h} \ldots \ldots \ldots \ldots \ldots \ldots \ldots 271$

Figure 6.4 Effect of load ratio $R$ on the maximum strain energy release rate $G_{\max }$ at fatigue threshold ........................................... 271

Figure 6.5 The prediction from Klesnil and Lukas's model for the relationship between

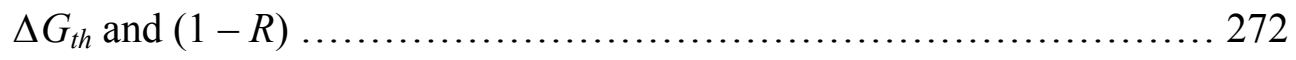




\section{CHAPTER 1}

\section{INTRODUCTION}

\subsection{Background}

For applications in civil infrastructure rehabilitation and new construction, wood is being reinforced with externally bonded laminates and fabrics of fiber-reinforced polymer (FRP) composites. Two types of FRP reinforcements for wood are being used: face bonding of FRP laminates to wood members, such as for commercial production of reinforced glued-laminated timber beams, and either bonding of FRP fabrics or wet lay-up by filament winding of wood cores, such as for applications as railroad ties and utility posts. The potential benefits of external reinforcement with bonded FRP composites have been demonstrated through several studies, and the field implementation of this technology has been successfully proven in numerous projects. However, there is a concern with the long-term performance of the interface bond, since an inadequate bond strength and integrity can render the reinforcement ineffective and lead to premature failure of the structure (Davalos and Qiao 2001). The performance of FRP-wood bonded interfaces under static and environmental loads has been explored extensively by Davalos et al. (1997, 1998a, 1998b, 2000a, 200b) and Qiao and Davalos (2000), and as an extension of this effort, the present study will examine, for the first time, the fatigue behavior of FRPwood bonded interfaces. 
Madabhusi-Raman (1995) and Trimble (1999), previous graduate students advised by Professor Davalos, conducted extensive work on interface fracture of bonded woodwood and FRP composite-wood materials. Their work is described briefly next.

Madabhusi-Raman (1995) studied the performance of red maple wood and pultruded phenolic laminate bonded interfaces under static loading, for wood-wood and wood-FRP combinations. He presented a numerical method based on the Rayleigh-Ritz solution to design the complex shapes of CDCB specimens for dissimilar materials. The CDCB specimen was modeled as a cantilever beam to obtain its compliance for a given crack length, and first-order shear deformation theory was used to account for shear deformation, which is important for anisotropic materials such as wood and composites. Each half of the CDCB specimen consisted of a constant cross-section adherend and a bonded contoured section manufactured from wood composites. The resulting shape of the exact contour depends on the geometric and the material properties of the contoured portion and the adherends. The exact contour has a convex shape. The effort and cost involved in accurately manufacturing such a complex shape can be significant. To facilitate manufacturing of the test specimen, a linear function was used to approximate the contour obtained by the Rayleigh-Ritz method. The constant compliance rate-change with respect to certain crack length was verified by an experimental and numerical (Finite element and Rayleigh-Ritz methods) study. The mode-I strain energy release rates for wood-wood and wood-FRP bonded interfaces were determined using the linear slope CDCB specimen. Madabhusi-Raman (1995) concluded that the CDCB specimen with a linear slope is suited for hybrid materials bonded interface testing. 
Trimble (1999) studied the effects of several parameters: surface texture of the adherends, types of adhesives, open/close assembly time, applied clamping pressure, and the usefulness of primers to improve the FRP-wood interface bond strength. The modified ASTM D 2559 method was used to evaluate the interface performance of both phenolic FRP and epoxy FRP bonded to red maple wood. The ASTM D 2559 standard consists of three wet-dry cycles (The details are given by Trimble, 1999). The best combinations of parameters suggested for phenolic FRP-red maple wood interface bond were RF adhesive without primer, 210 psi clamping pressure, and 5/30 minutes open/closed assembly time.

\subsection{Objective and Scope}

Structural components used in practical applications are subjected to variable amplitude fatigue loads. The scientific knowledge gathered on the constant amplitude cyclic loading could be applied to the more realistic situations involving varying amplitudes of cyclic load. The objective of this study is to develop a combined analytical and experimental fracture mechanics method to evaluate the fatigue behavior of phenolic FRP and red maple wood bonded interfaces under constant cyclic load conditions. The possible effect of loading variables (load ratio, waveform and frequency) on crack propagation rate is studied. The fatigue threshold is determined towards the end of this study and the possible load ratio effect on fatigue threshold is investigated. The study of constant amplitude fatigue on phenolic FRP-red maple wood bonded interfaces provides valuable insights into the mechanical processes by which fatigue failure occurs for the real structures or member under varying amplitude cyclic loading. The proposed eight major tasks are: 
1. To develop test methods for determination of the crack growth rate for phenolic FRP-red maple wood bonded interfaces under constant cyclic loading conditions.

2. To conduct constant cyclic loading fatigue tests and determine the possible effect of the loading variable, such as waveform, load ratio and frequency on phenolic FRPred maple wood bonded interfaces.

3. To develop an analytical model to be able to explain the possible effect of waveform, load ratio and frequency on phenolic FRP-red maple wood bonded interfaces.

4. To characterize and provide new knowledge of the fatigue process of phenolic FRP-red maple wood bonded interfaces.

5. To develop test methods for determination of the fatigue threshold value of the strain energy release rate for phenolic FRP-red maple wood bonded interfaces under constant cyclic loading conditions.

6. To determine the threshold value of the strain energy release rate of phenolic FRPred maple wood bonded interfaces.

7. To investigate the possible load ratio effect on the threshold value of the strain energy release rate of the phenolic FRP- red maple wood bonded interfaces. 
8. Finally, to recommend practical guidelines for fatigue tests of hybrid material bonded interfaces.

In this study, new and promising techniques to characterize the fatigue behavior of phenolic FRP-red maple wood bonded interfaces are proposed, and fundamental aspects for proper analytical predictions and experimental verifications are investigated. Based on this study of the performance of phenolic FRP-red maple wood bonded interfaces, practical guidelines are suggested for further consideration as standardized methods.

\subsection{Problem Significance}

There is a worldwide need to rehabilitate and improve civil infrastructures. To this end, new construction materials and methods are being intensely investigated to alleviate current problems and provide better and more reliable future services. Recent studies (Davalos and Barbero, 1993) estimate total needs in the U.S. of three trillion dollars in the next decade to bring industrial and public works to safe operational levels. This national need presents a unique opportunity for research and development of wood/fiber-reinforced polymer (FRP) materials that can provide longer service life and lower life cycle costs than

conventional materials, such as concrete and steel. New FRP-wood hybrid materials for high volume construction applications are being developed from low cost constituents, such as wood composites and synthetic fibers and resins. 
Similar to wood products, the favorable attributes of FRP-wood materials are lightweight, corrosion resistance, nonmagnetic, and nonconductive properties. In addition, FRP-wood materials exhibit excellent energy absorption properties, suitable for seismic response, high strength, fatigue resistance, durability, competitive costs based on load capacity per unit weight, ease of handling, transportation, and installation. The potential applications of FRP-wood materials include bridges, railroad ties, poles, long span roof structures, buildings, highway noise barriers, fire barriers in coal mines, storage structures exposed to salts and chemicals, and many others.

The commercial development and implementation of FRP-wood materials can contribute to alleviate major problems adversely affecting infrastructure deterioration worldwide, such as corrosion of steel, high labor costs, energy consumption, environmental pollution, and devastating effects of natural hazards. Two immediate potential applications being explored in the U.S. for FRP-wood products are highway bridges and railroad ties. With nearly $40 \%$ of the approximately 600,000 highway bridges in need of repair or replacement, FRP-wood materials can efficiently used in bridge construction, particularly for improving rural transportation and revitalizing rural economies. Similarly, considering that over 12 million railroad timber ties are replaced annually at a cost of about 500 million dollars, the combination of wood ties with FRP can offer improved and longer performance.

Several studies in the U.S. and abroad have demonstrated the significance increases in strength and stiffness that can be achieved by FRP reinforcement of wood. Recently, an 
FRP reinforcement technique for Glulam has been patented, and the process has been implemented commercially. Similarly, several parallel efforts are being invested in qualifying polymer materials for reinforcement of wood. Current research on wood reinforcement has focused on the use of FRP strips or fabrics bonded to wood with several adhesives, with particular emphasis on phenolic FRP composites and resorcinol formaldehyde wood adhesives. Since the delamination of the interface bond can lead to premature failure of FRP-wood structural members, there is a concern with the long-term reliable performance of the interface bond, which is crucial in design and durability of FRP-wood products. Unfortunately, there are no investigations reported for bonded interfaces of hybrid FRP-wood composites under cyclic loading. There is a need to develop combined theoretical and experimental methods to characterize the performance of FRP-wood interface bonds under service fatigue loading conditions.

Fatigue failure of materials and bonded interfaces is a very complex topic, and yet a quite important issue in engineering practices, since most structures are subjected to both applied and environmental cyclic loading during their service life. The fatigue failure process has not been completely understood and there is no single accepted criterion or law to explain fatigue phenomena. However, the application of linear elastic fracture mechanics concepts has resulted in reasonable and useful explanations of fatigue failure, especially for crack propagation rate measurement and fatigue life prediction. By modifying the original Paris Law equation, we can efficiently evaluate the fatigue behavior of phenolic FRP-wood interface bond-line, and this method can further be used for other similar studies of interface fracture of dissimilar materials. Thus, the findings of this study 
can eventually lead to the development of guidelines for hybrid material bonded interface design under constant cyclic loading conditions.

\section{$1.4 \quad$ Fatigue Overview}

A concise review of the fatigue process and methods to characterize fatigue phenomena are presented.

\subsubsection{Three stages of fatigue process}

It is generally accepted that there are three stages that characterize the fatigue process: (a) Region I or near threshold region, (b) Region II or intermediate region, and (c) Region III or high growth rate region, as illustrated in Fig. 1.1 (Suresh 1998).

The first stage (Region I) of fatigue process is called near-threshold region. The fatigue crack growth rate in this stage is very slow, and the increment of average crack extension is less than $10^{-6} \mathrm{~mm} / \mathrm{cycle}$. For all practical purposes, the crack growth rate in this region sometimes is simplified to be zero. In Region I, the stress intensity factor range $\Delta K$ approaches the fatigue crack growth threshold $\Delta K_{t h}$. Below the fatigue threshold, a crack grows at undetectable rates; above the fatigue threshold, the crack growth is classified into Region II, in which the Power Law equation is applicable. An operational definition for $\Delta K_{t h}$ is commonly used in terms of a maximum crack growth rate (typically $10^{-8} \mathrm{~mm} /$ cycle), based on the accuracy of the crack monitoring system and the number of elapsed cycles (Suresh 1998). The first stage has some features. For example: fracture 


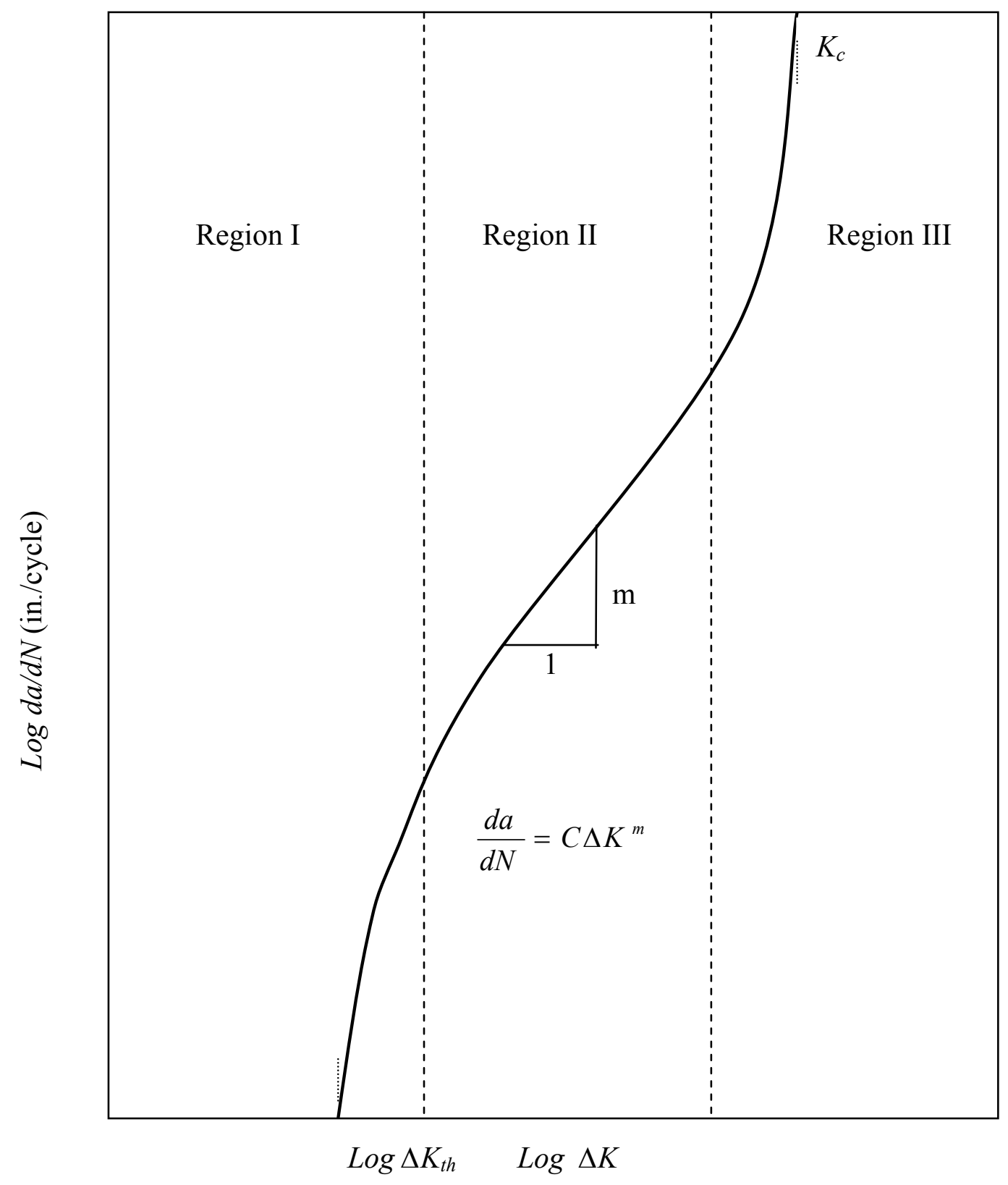

Figure 1.1 A schematic illustration of three stages of a fatigue process (Suresh 1998) 
surface consists of faceted and serrated cracks, load ratio and environmental effects are large, and crack closure levels are high. There have been many research studies on the near threshold fatigue of engineering materials. In this study, the fatigue threshold of phenolic FRP-red maple wood bonded interface is determined, and the influence of load ratio on the fatigue threshold is investigated as well.

The intermediate stage (Region II), also called Paris or linear region, occupies the major part of the fatigue life. The fatigue crack growth of this region in engineering materials has been the subject of most extensive research. Paris, Gomez and Anderson (1961) and Paris and Erdogan (1963) were apparently the first to discover the Power Law relationship for fatigue crack growth in Region II. They proposed an exponent of $m=4$, which was in line with their experimental data. However, subsequent studies over the past four decades have shown that $m$ is not necessarily four, but ranges from 2 to 5 for metals, and it is much higher for composite materials. However, there are no values of $m$ reported for the bond-line or interface of wood-FRP and concrete-FRP.

The high growth rate stage (Region III) has a very high $\Delta K$ value. The maximum stress intensity factor value $K_{\max }$ approaches the critical value of the stress intensity factor $K_{c}$. The fatigue crack growth rate in this region is much higher than in Region II, and a catastrophic failure occurs at the end of this stage.

Dowling and Thangjithan (2000) replaced the asymptotes in Fig. 1.1 with vertical lines (see Fig. 1.2) in an attempt to simplify the established methodology of fatigue 


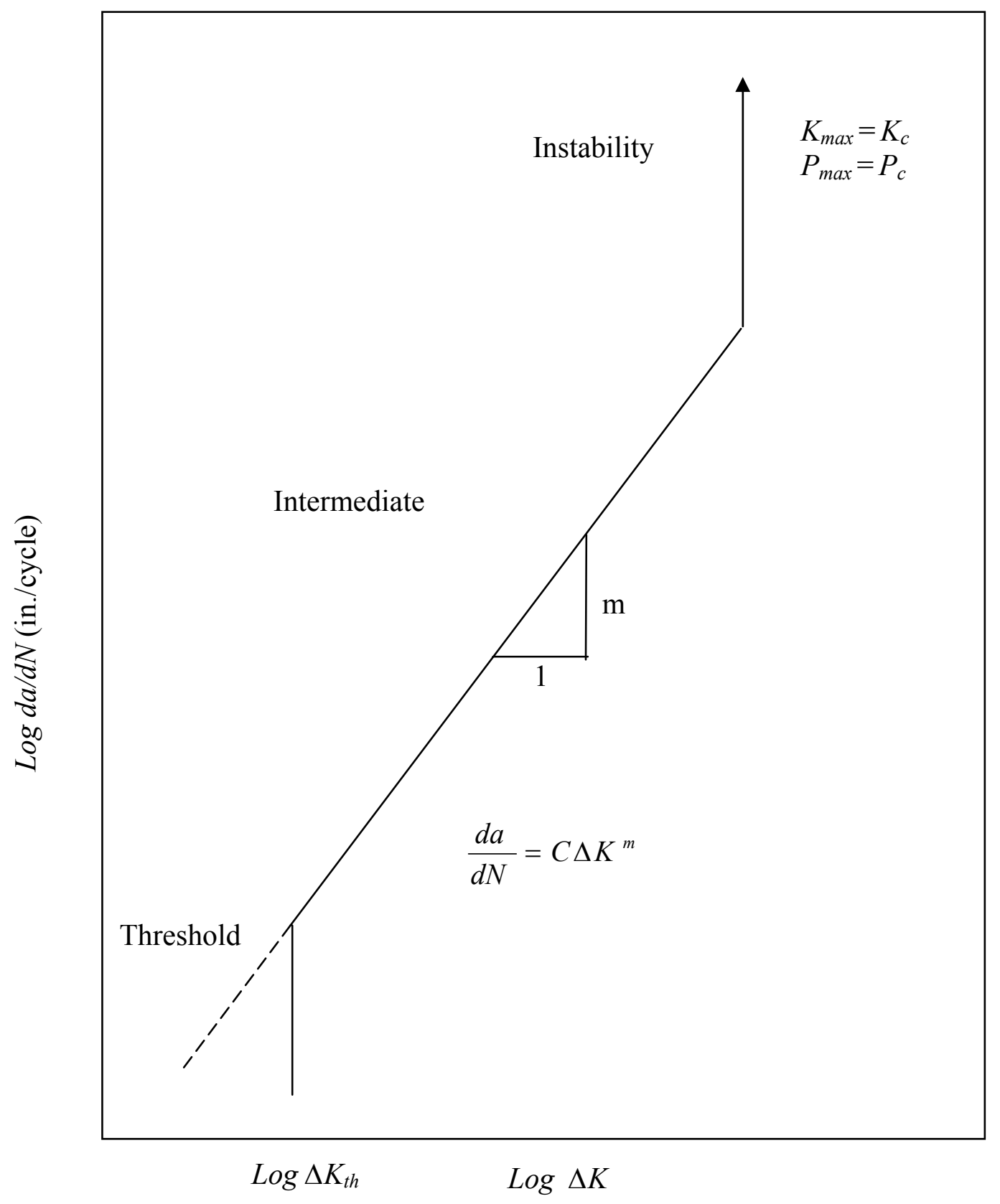

Figure 1.2 An approximate representation of the fatigue process by Dowling et al. (2000) 
analysis for straightforward engineering applications. The crack growth rate is taken to be zero when $\Delta K$ is less than or equal to $\Delta K_{t h}$, and the catastrophic failure is assumed to occur when the maximum stress intensity factor or maximum load is equal to the critical stress intensity factor or critical load. Thus, they suggested a conservative option that ignores the threshold region denoted by the dashed line (see Fig. 1.2). However, as indicated by several authors, caution is needed in assuming that an absolute fatigue threshold exists, since overload interaction effects may decrease the value of $\Delta K_{t h}$, and for small cracks $\Delta K_{t h}$ may be lower than that for long cracks. 


\subsubsection{The analysis methods for fatigue problems}

Ever since people notice the importance of failure caused by cyclic loading, researchers have been trying to find a way to explain the fatigue phenomenon. There are several main methods accepted by most researchers: (1) the artificial neural network method, (2) the statistical based method, (3) the damage accumulation method, and (4) the fracture mechanics based method.

Artificial neural network (ANN) has become a new branch of computing, which tries to mimic the structure and operations of biological neural systems. An ANN is able to learn by examples and does not have to know the theory behind a phenomenon. This quality is useful to describe problems where the relationships of inputs and outputs are not clear enough or the solutions are hardly formulated in a short time. An ANN has been applied to a wide range of fields, beginning with pattern recognition, in particular for applications to fracture studies and extending to failure analysis, non-destructive testing, and static and fatigue tests. An artificial neural network based strategy can effectively manage fatigue parameter information, "learn" relationships between factors and the results through sample data, and predict outputs based on input sets for cases beyond experimental results. A detailed literature review on application of the artificial neural network (ANN) approach to fatigue problems is given in the author's doctoral qualifying examination (Jia 2002). The characteristics of problems that ANN could be used for are: (1) the data set is complex and noisy and not complete; (2) there is no accurate solution to the problems by known analytical or theoretical methods; (3) experimental tests are time consuming and costly, and (4) there is a big data set available. Fatigue tests have all of the 
above characteristics; it is feasible to apply the artificial neural network approach to modeI fatigue tests. Therefore, there is a possibility of future work on exploring the ANN approach to fatigue problems, especially for wood-FRP bonded interface.

There are a number of uncertain factors in the analysis of fatigue problems. Such uncertainties are analyzed using known statistical methods to obtain the reliability or probability level of failure. There are many references to review these statistical methods to fatigue, such as ASTM STP 744. One example commonly adopted to statistically characterize the fatigue is the Weibull distribution. The objective of incorporating probabilistic analysis into fatigue design is to ensure a low probability to cause failure. However, the statistical method is generally applied to the stress-life analysis. An endurance limit is needed to be established first based on experiments. We did not use the statistical method in the present investigation, because of limited time and resources, and also because we are concerned only with loading variable effects on crack propagation rate and fatigue threshold.

In the damage accumulation method, fatigue failure is believed to be a process of gradual accumulation of damage. The strength of materials or structures subjected to unfavorable mechanical or environmental conditions decreases due to the accumulation of micro-structural damage. In recent years, the concept of damage tolerant design has developed for fatigue failure and gained increasing acceptance. Such design requires that the structure should retain adequate strength and stiffness in the presence of damage, until the damage exceeds a certain level. To properly evaluate the damage level when designing 
reliable structures it is necessary to formulate the damage phenomenon in terms of mechanics. During the last two decades the basic principles of continuum damage mechanics were formulated and some special problems were solved. Since Kachanov (1985) published the first book about the damage mechanics, continuum damage mechanics is rapidly developing a branch of fracture mechanics.

Recently, a number of workers have independently attempted to develop models, which relate the micro-structural change to damage growth, residual strength decrease and fatigue life. Poursartip et al. (1986) developed a damage model, which assumes a relationship between fatigue damage and changes in the moduli of laminates. Experimental data are used to determine a damage function, $f(D, R, \Delta \sigma)$, which describes how the rate of accumulation of damage, $d D / d N$, depends on the current level of damage, $D$, the load ratio, $R$, and the stress amplitude, $\Delta \sigma$. This approach can be integrated for stress history, $\Delta \sigma(N)$, to give, after $N$ cycles, the current level of damage, $D$. This approach also can be applied to fatigue life prediction. The basic idea of this approach is demonstrated in the following. The damage function is:

$$
d D / d N=f(\Delta \sigma, R, D)
$$

It can be integrated to give the fatigue life, $N_{f}$ (the number of cycles to failure) as

$$
N_{f}=\int_{D_{i}}^{D_{f}} \frac{d D}{f(\Delta \sigma, R, D)}
$$


where $D_{i}$ and $D_{f}$ stand for the initial and final damage. If a relation exists between the axial Young's modulus, $E$, and the damage, $D$,

$$
E=E_{0} g(D)
$$

where $E_{0}$ is the undamaged modulus, then we have that

$$
\frac{1}{E_{0}} \frac{d E}{d D}=g^{\prime}(D)
$$

where $g^{\prime}$ means the derivative of $g$ with respect to $D$. Using the chain rule for differentiation, we find:

$$
\frac{1}{E_{0}} \frac{d E}{d N}=g^{\prime}\left(g^{-1}\left(\frac{E}{E_{0}}\right)\right) f\left(\Delta \sigma, R, g^{-1}\left(\frac{E}{E_{0}}\right)\right)
$$

where $g^{-1}$ is the inverse of $g$ :

$$
D=g^{-1}\left(\frac{E}{E_{0}}\right)
$$

If data can be gathered for $E / E_{0}$ as a function of number of cycles, then $g(D)$ is known. To do so, a parametric study is conducted: (1) for a range of values of $\Delta \sigma$ at constant $E / E_{0}$ and 
$R$, (2) for a range of $R$ at constant $\Delta \sigma$ and $E / E_{0}$, (3) for a range of $E / E_{0}$ at constant $\Delta \sigma$ and $R$. Thus, the function $f(D, R, \Delta \sigma)$ can be determined experimentally

$$
f(\Delta \sigma, R, D)=\frac{1}{g^{\prime}\left(g^{-1}\left(E / E_{0}\right)\right)} \frac{1}{E_{0}} \frac{d E}{d N}
$$

For a carbon fiber composite laminate, CRC (45/90/-45/0), Poursartip et al. (1986) gave the empirical equation:

$$
\frac{d D}{d N}=9.189 \times 10^{-5}\left(\frac{\Delta \sigma}{\bar{\sigma}_{T S}}\right)^{6.393}
$$

This method was adopted in the beginning of this project, but it was not successful since the damage mechanics method is more suitable for solids than interface bonds. Therefore, the fracture mechanics based method is adopted in this study, and the detailed literature review follows.

During the past four decades, the mechanical treatment of fatigue crack propagation has been advanced by the developments in the field of fracture mechanics, by the introduction of servo-hydraulic testing machines, and by the availability of scanning electron microscopy and other novel techniques for microscopic observations. The relationship between crack growth rate and fracture toughness was first established by Paris et al. in the 1960's, who introduced the well-known Power Law (or Paris Law) equation, which states that the crack growth rate $d a / d N$, where $a$ is the crack length and $N$ 
is the number of cycles, depends mainly on the amplitude of the stress intensity factor $\Delta K$ (Paris, Gomez and Anderson 1961; and Paris and Erdogan 1963):

$$
\frac{d a}{d N}=C \Delta K^{m}
$$

where $C$ and $m$ are empirical constants dependent on materials, loading variables, environments and other factors, which need to be determined experimentally. Paris proposed an exponent $m=4$ in agreement with his experimental data for aluminum alloys 2024-T3 and 7075-T6. Subsequent researchers over decades showed that the exponent $m$ in equation (1.9) is not necessarily equal to 4 , and the value may vary depending on the type of materials. It was found that the exponent $m$ ranges from 2 to 5 for metals, is higher than 4 for many polymers and plastics and even much higher for composite materials.

The Power Law equation (1.9) only represents the relation of $d a / d N$ and $\Delta K$ in the intermediate rate region (region II) shown in Figs. 1.1 and 1.2. It does not represent the relationship between $d a / d N$ and $\Delta K$ in region I and region III. In region I, there is a threshold intensity range $\Delta K_{t h}$ below which the crack growth rate is practically zero. In region III, a huge acceleration of crack growth takes place, and unstable fracture starts at the maximum stress intensity factor approaching to the critical fracture toughness. The characteristics of each stage are described in section 1.4.1. Many researchers have developed modified Paris Law equations that model all or part of sigmoidal variation in the relationship of $d a / d N$ and $\Delta K$. Although some of these equations are based on some physical considerations, a number of them are empirical. 
A semi-empirical modified Paris Law equation for Regions II and III were provided by Weertman (1966):

$$
\frac{d a}{d N}=\frac{C \Delta K^{4}}{K_{c}^{2}-K_{\max }^{2}}
$$

This equation can be made more general with a variable exponent, $m$, on $\Delta K$. In 1967 , Forman et al. proposed the following alternative relationship for Regions II and III:

$$
\frac{d a}{d N}=\frac{C \Delta K^{m}}{(1-R) K_{c}-\Delta K}
$$

Eq. (1.9) assumes that $d a / d N$ depends only on $\Delta K$ and ignores the importance of mean level of stress intensity factor $K_{\text {mean }}$ or the stress ratio $R=K_{\min } / K_{\max }$, while the relationship in Eq. (1.11) accounts for the stress ratio $R$ effect; this expression has found some applications in metals with reasonable success. Interestingly, if we rewrote equation (1.11) in the following form:

$$
\frac{d a}{d N}=\frac{C \Delta K^{m-1}}{\frac{K_{c}}{K_{\max }}-1}
$$


the crack growth rate $d a / d N$ would become infinite as the maximum stress intensity factor $K_{\max }$ approached the critical stress intensity factor $K_{c}$. That is, the crack growth rate $d a / d N$ is asymptotic to $K_{\max }=K_{c}$. This same idea is implicit in Eq. (1.10).

However, it should be noted that both the equations (1.10) and (1.11) did not take into account the value of the fatigue threshold $\Delta K_{t h}$. A simple and effective way to account for the fatigue threshold was suggested by Klesnil and Lukas (1972a):

$$
\frac{d a}{d N}=C\left(\Delta K^{m}-\Delta K_{t h}^{m}\right)
$$

In this case, the threshold is a fitting parameter to be determined experimentally. A similar type of equation proposed by Liu (1964) is expressed as

$$
\frac{d a}{d N}=C\left(\Delta K-\Delta K_{t h}\right)^{m}
$$

It must be pointed out, however, that the fatigue threshold $\Delta K_{t h}$ is strongly dependent on the load ratio $R$ for many materials; this may cause some problems in Eq. (1.13) and Eq. (1.14) when dealing with the load ratio effect.

Some researchers attempted to describe not only the stress ratio effect but also the entire range of loading spectrum, and take into account both the threshold $\Delta K_{t h}$ and the 
critical stress intensity factor $K_{c}$. For example, Chu (1974) proposed the following empirical equation in a very elaborate way

$$
\frac{d a}{d N}=\frac{C\left(\Delta K^{m_{1}}-\Delta K_{t h}^{m_{2}}\right)}{\left[(1-R) K_{c}-\Delta K\right]^{m}}
$$

More recently, Arad et al. (1971) developed a simplified crack propagation model for a range of polymers

$$
\frac{d a}{d N}=C\left(K_{\max }^{2}-K_{\min }^{2}\right)^{m}
$$

This model has been applied to some metallic materials, such as aluminum alloys (2024T3 and 7075-T6) and steel (medium carbon steel and cold rolled mild steel), with considerable success reported by Branco et al. (1975a).

Ever since Paris first applied fracture mechanics to the problem of fatigue crack propagation, the stress intensity factor has been widely used as a loading parameter in correlating the fatigue crack growth rate. About ten years later after Paris's study, researchers had proposed some other parameters such as crack tip opening displacement (CTOD), plastic zone size (PZS), strain energy release rate $G$, and $J$-integral in their Power Law equations. Two examples from Tanaka (1989) are:

$$
d a / d N=C(\triangle C T O D)^{m}
$$




$$
d a / d N=C(P Z S)^{m}
$$

Similarly, Mostovoy (1975) related the range of the strain energy release rate to crack propagation rate, as

$$
d a / d N=C(\Delta G)^{m}
$$

Under Mode I loading, $\Delta G$ is proportional to $K_{\max }^{2}-K_{\min }^{2}$, with appropriate coefficients.

The prediction of the crack growth rate from stress intensity factor range $\Delta K$ is no longer valid for the elastic-plastic and gross plasticity conditions. Dowling and Begley (1976) first applied the $J$-integral to fatigue crack growth rate and formulated a Power Law equation in $\Delta J$, as

$$
d a / d N=C(\Delta J)^{m}
$$

All the above modified Power Law equations are listed in Table 1.1 for convenient reference. The fitting parameters, $C$ and $m$, do not necessarily have the same values or units in the various crack growth equations of Table 1.1. 
Table 1.1. The modified Paris Law equations for fatigue crack propagation

\begin{tabular}{ccc}
\hline Modified Paris Law equation & Authors & Year \\
\hline$\frac{d a}{d N}=\frac{C \Delta K^{4}}{K_{c}^{2}-K_{\max }^{2}}$ & Weertman & 1966 \\
$\frac{d a}{d N}=\frac{C \Delta K^{m}}{(1-R) K_{c}-\Delta K}$ & Forman et al. & 1967 \\
$\frac{d a}{d N}=C\left(\Delta K^{m}-\Delta K_{t h}^{m}\right)$ & Klesnil et al. & 1972 \\
$\frac{d a}{d N}=C\left(\Delta K-\Delta K_{t h}\right)^{m}$ & Liu & 1964 \\
$\frac{d a}{d N}=\frac{C\left(\Delta K^{m_{1}}-\Delta K_{t h}^{m_{2}}\right)}{\left[(1-R) K_{c}-\Delta K\right]^{m}}$ & & 1971 \\
$\frac{d a}{d N}=C\left(K_{\max }^{2}-K_{\min }^{2}\right)^{m}$ & Chu & 1975 \\
$d a / d N=C(\Delta G)^{m}$ & Arad et al. & 1976 \\
$d a / d N=C(\Delta J)^{m}$ & Mostovoy et al. &
\end{tabular}

\subsection{The Contoured Double Cantilever Beam (CDCB) Specimen}

A fracture toughness test measures the resistance of a material to crack extension. A variety of organization throughout the world published standardized procedures for fracture toughness measurements, including the American Society for Testing and Materials (ASTM), the British Standards Institution (BSI), the International Institute of 
Standards (ISO) and Japan Society of Mechanical Engineers (JSME). ASTM published the first standards for stress intensity factor $K$ and $J$ contour integral testing in 1970 and 1981, respectively, while BSI published the first CTOD test method in 1979.

There are many types of specimens that characterize the fracture initiation and crack growth, although no single standard allows all these configurations, and the design of a particular specimen type may vary between standards and researchers. The configurations that are currently standardized include the compact specimen, the single edge notched bend geometry, the arc-shaped specimen, the disk specimen, the middle tension panel, and the double cantilever beam specimen. On the other hand, there are a number of specimen configurations that are used in research, but have not been standardized yet. However, some of these specimen configurations have the potential that could be superior to the standardized specimen configurations.

A method of crack-line loading was originally developed to evaluate the fracture toughness of structural adhesives. The specimen commonly used in this work is referred to as a double cantilever beam (DCB) specimen. A DCB specimen consists of a pair of long rectangular boxes bonded together with the target adhesive that needs to be tested. This specimen is also being used to test monolithic materials by cutting side grooves in the same direction of pre-crack. The strain energy release rate $G_{I}$, which is a measure of the fracture toughness of an interface bond under Mode-I loading, is given as

$$
G_{I}=\frac{P^{2}}{2 b} \frac{d C}{d a}
$$


where $P$ is the applied load, $b$ is the thickness of the specimen, $d C / d a$ is the rate of compliance change with respect to crack length. Fracture occurs at a critical load, $P_{c}$, corresponding to critical strain energy release rate, $G_{I c}$. As indicated by Anderson (1994), the use of $G_{I c}$ as a measure of fracture toughness is equivalent to the use of a critical stress intensity factor $K_{I c}$. The accuracy of measuring strain energy release rate $G$ by the use of Eq. (1.21) is greatly restricted by the accuracy of determining the value of $d C / d a$. First, with the DCB specimen, which consists of a pair of beams rather than tensile elements, the crack opening displacements are large while the critical load is small. Hence, reliable compliance measurements are often restricted by the accuracy of the crack opening displacement measurements. Second, the value of $d C / d a$ in Eq. (1.21) depends on the accuracy of the crack length measurement. As a practical matter, it is generally a difficult task to measure simultaneously the critical load and crack length for each load step.

The difficulty of measuring the crack length can be avoided if a specimen is designed to provide a constant compliance rate-change with respect to crack length. In this case, the specimen is known as the Contoured Double Cantilever Beam (CDCB) specimen. The $d C / d a$ value of a CDCB specimen is a constant. The relationship between load $P$ and strain energy release rate $G$ is independent of crack length $a$. In general, to obtain a CDCB specimen with constant $d C / d a$, it is convenient to vary the specimen height with crack length. 
Mostovoy et al. first proposed the concept of the contoured double cantilever beam (CDCB) specimen in 1967, in order to simplify the experimental procedure. By equating the compliance rate change to a predetermined constant value, they obtained the height of the cross section for different crack lengths of a CDCB specimen. From the relationship of the crack length and the height of the cross section, the contour of the CDCB specimen was defined. These authors also indicated that a linear shape could approximate the contoured shape of a CDCB specimen.

For the evaluation of mode-I fracture toughness under cleavage loading, the CDCB specimen offers a great advantage. By contouring the DCB specimen, one can obtain the constant $d C / d a$ value ( $C=$ compliance, $a=$ crack length), and the strain energy release rate, $G$, is dependent only on applied load over a significant crack length range. Moreover, the $\mathrm{CDCB}$ specimen has the ability to allow measurements of fracture toughness for crack initiation as well as arrest. These qualities can reduced the experimental difficulty of measuring fracture toughness and simplified the study of sub-critical crack growth typified by fatigue and stress corrosion cracking. A detailed literature review on the applications and design of the CDCB specimen is given in section 1.5.1.

Rcently, Davalos and his coworkers at West Virginia University have developed a CDCB specimen designed by the Rayleigh-Ritz method. The CDCB specimen is contoured to achieve a constant rate of compliance change with respect to crack length, $d C / d a$. The details of the specimen design and applications to wood-FRP interface fracture studies at West Virginia University are given in section 1.5.2. 


\subsubsection{Applications of the CDCB specimen}

Liu-Nash et al. (1997) conducted the near-threshold fatigue crack propagation tests with the contoured double cantilever beam (CDCB) specimen using the standard beam formula for compliance. In her CDCB specimen design, the stress intensity factor $K$ is given by:

$$
K=P \sqrt{\frac{E}{2 B} \frac{\partial C}{\partial a}}
$$

From the standard beam formula for compliance, she obtain:

$$
C=\frac{\delta}{P}=\frac{2 a}{2 E I}=\frac{8 a^{3}}{E B h^{3}}
$$

Where $C=$ compliance $(\mathrm{mm} / \mathrm{N}) ; \delta=$ displacement $(\mathrm{mm}) ; P=\operatorname{applied}$ load $(\mathrm{Pa}) ; E=$ elastic modulus (MPa); $I=$ moment of inertia; $B=$ beam width $(\mathrm{mm})$; and $h=$ beam height $(\mathrm{mm})$. The compliance change with crack length was obtained by differentiating Eq. (1.23):

$$
\frac{d C}{d a}=\frac{24 a^{2}}{E B h^{3}}=\frac{3 a^{2}}{h^{3}}\left(\frac{8}{E B}\right)
$$

Consequently, a geometry term, $m$, is defined as: 


$$
m=\frac{3 a^{2}}{h^{3}}+\frac{1}{h}
$$

where $m=$ specimen geometry factor $(1 / \mathrm{mm}), 1 / h=$ shear correction term $(1 / \mathrm{mm})$. She calculated the geometry parameter $m=2.559 \mathrm{~mm}^{-1}$ for her initial experiments, and the final geometry parameter that she used for the ASTM A710 HSLA steel CDCB specimen was $m$ $=0.0524 \mathrm{~mm}^{-1}$.

Marcus and Sih (1971) used a tapered double cantilever beam (TDCB) specimen to study the Ti alloy stress corrosion in methanol- $\mathrm{NaCl}-\mathrm{H}_{2} \mathrm{O}$ solution. The linear dependence of the compliance with crack length was experimentally determined. The advantages of the TDCB specimen in stress corrosion is the ease of locating the region of the running crack formed when there was explicitly defined load, temperature, and the applied electrochemical potential values. Also it was reported that the TDCB specimen supplied more consistent data than was generally observed in stress corrosion studies.

El-Senussi and Webber (1989) studied delamination of carbon fiber-reinforced plastic laminates under both static and cyclic loading with TDCB specimens. The following three different methods: (1) area method, (2) compliance method, and (3) theoretical method, were used to evaluate the critical strain energy release rate. First, the area method gives the equation:

$$
G_{c}=1 / b \times(A / \delta a)
$$


where $b$ is the thickness of the specimen, $a$ the crack length, $A$ is an "envelope area" which refers to the area enclosed by the load line and axis X segment from the point of initial load to the point of arrest load, as shown by the shaded region in Fig. 1.3.

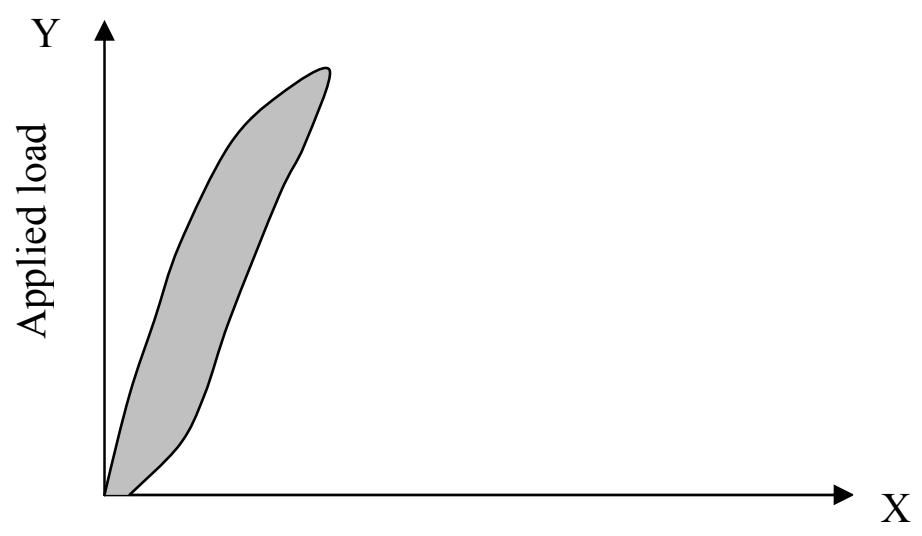

Crack opening displacement

Fig. 1.3 A schematic of the envelope area for the area method

Secondly, the compliance method gives:

$$
G_{c}=\left(P_{c}^{2} / 2 b\right) d C / d a
$$


where $b$ is the thickness of the specimen, $d C / d a$ is the compliance change with crack length, and $P_{c}$ is the critical load. Third, the theoretical method gives:

$$
G_{c}=P_{c}^{2} / E b^{2} h_{a}^{2}\left[12 a^{2} / h_{a}+51.6 e \lambda / \pi\left(a / h_{a}\right)+3.75 e \lambda\right]
$$

This equation is derived from simple bending theory considering the effect of direct bending and shear strains along the specimen's cross section through the crack tip. Very good agreement for the critical strain energy release rate was achieved between experiment and theory with the above three methods. The fatigue tests were carried out in load control mode on a servo hydraulic Instron machine with a frequency of $6 \mathrm{~Hz}$. The plots of crack length versus number of cycles showed that there existed an approximate linear behavior after about a hundred thousand cycles. This could be the result of the strain energy release rate independent on the crack length. The $\Delta P$ was held constant, and therefore, the $\Delta G$ would also be kept constant. The number of samples tested was not enough to obtain fatigue crack growth equations. However, their experiments implied the possibility that a simple Power Law type equation could be used to predict the fatigue delamination growth rate. Several papers that followed showed that we could use T/CDCB specimens for cyclic loading tests.

Tsuji, Nakajima and Kondo (1992) carried out fatigue tests on a pressure vessel steel SA533B-1. The applied load was of triangular waveform at a frequency of $0.5 \mathrm{~Hz}$ and load ratio of 0.1. The compact tension (CT) and contoured double cantilever beam (CDCB) specimens, corresponding respectively to $\Delta K$ increasing and $\Delta K$ constant, were used. The 
linear relationship between crack length and number of cycles for the CDCB specimen was confirmed experimentally. The variability of the measured values between the CT and CDCB specimens was examined statistically. The correlation coefficients of $d a / d N$ versus $\Delta K$ for the data obtained from $\Delta K$ constant type tests were always larger than those for the data from $\Delta K$ increasing type tests. The standard deviations about the regression line of Power Law equation and about the mean $d a / d N$ values were evaluated, and the results showed that the standard deviations for the CDCB specimen were much smaller than those for the CT specimen. The possible sources for variability of the data by $\Delta K$ increasing type tests were pointed out as heterogeneity in the microstructure of the material as well as precision of crack length measurement in the low $\Delta K$ region and change of the fracture mode in the high $\Delta K$ region. These authors concluded that $\Delta K$ constant type tests were superior to $\Delta K$ increasing type tests from the viewpoint of variability of the data.

Russell and Street (1988) used a double cantilever beam (DCB) specimen together with an attached elastic spring in order to achieve constant strain energy release rate condition. The reason they did not consider tapered or contoured DCB specimen was the difficulty of specimen fabrication for their purposes. Since the condition of strain energy release rate independent of crack length was achieved for this specimen, it can be considered to be the same as a CDCB specimen. During Mode I constant load cycling, the bridging effect leads to an increase of fracture resistance, and for most conventional specimens, the strain energy release rate $G$ changes as the crack extends, and in this case any decrease in $d a / d N$ due to fiber bridging is obscured by the change of $\Delta G$. In contrast, the CDCB specimen that exhibits constant $\Delta G$ can successfully overcome this problem. 
The fatigue tests by Russell and Street (1988) were carried out at frequencies of 2.5 and 1 $\mathrm{Hz}$ with the load ratio of 0.05 . The crack growth rate was reported to decrease as the crack extended for all AS1/3501 and AS4/PEEK specimens. In addition to decline in $d a / d N$, a zone of bridged fibers was observed to form behind the advancing crack, and an increase in the Mode I fracture resistance was reported. The effect of raising temperature $\left(20^{\circ} \mathrm{C}\right.$ to $100^{\circ} \mathrm{C}$ ) in $\mathrm{AS} 1 / 3501-6$ was two fold. First, it resulted in an increase in the $d a / d N$ values, and second, it lowered the exponent of the $\Delta G$. A single relationship between crack growth rate and fracture resistance was given as

$$
\frac{d a}{d N}=\left[\frac{\Delta G}{G_{I R}}\right]^{n}
$$

where $G_{I R}$ is the instantaneous resistance to fracture.

Branco et al. (1975b) studied the effect of the mean stress intensity factor $K_{m}$ and the range of the stress intensity factor $\Delta K$ on fatigue crack growth rate in an aluminum alloy RR58. They used a CDCB specimen, which provided a constant stress intensity factor for all crack lengths. In order to comply with the condition of the constant $d C / d a$ value, the contoured shape was chosen having the form

$$
\frac{3 a^{2}}{h^{3}}+\frac{1}{h}=38
$$


where $h$ is the specimen height at the crack length $a$. The crack starter was a saw cut, and sharpened by a razor blade. A linear relationship between the crack growth and the number of cycles was obtained.

Mizutani and Iwatsu (1983) used a TDCB specimen to study the influence of external environments on fatigue crack growth in epoxy resin. The specimen was tapered as

$$
\frac{3 a^{2}}{h^{3}}+\frac{1}{h}=0.5 \mathrm{~mm}^{-1}
$$

to get the stress intensity factor independent on the crack length. A pre-crack that had a very small crack tip radius was formed by applying a cyclic load. A constant load test was carried out at a frequency of about $17 \mathrm{~Hz}$. Several kinds of organic solvents (n-Hexane, Ethyl acetate, Acetone, Ethanol, Methanol and n-Butanol) were used to simulate environmental effects on fatigue crack growth in epoxy. The original Paris Law was valid, and the material constant in air was given as

$$
\frac{d a}{d N}=1.2 \times 10^{-4} \times\left(\Delta K_{I}\right)^{6.5}
$$

They found that the crack growth rate in the presence of solvents decreased 1/4 to1/10 of that in air. The decrease of the crack growth rate in the solvents was considered to be due to large plastic deformation by penetration of solvent into the resin at the crack tip, and therefore, more fracture energy was needed to extend the crack. 


\subsubsection{Previous research at West Virginia University}

The information and writing style presented in this section follows closely those presented earlier by Professor Davalos and his graduate students (Madabhusi-Raman and Trimble) and his collaborators (Dr. Qiao). This overview is given in this section for the reader's benefit.

To test the strength of structural adhesives between dissimilar materials, it is convenient to use a CDCB specimen with constant-thickness adherends bonded to contoured portions made of a material that is easy to shape, such as wood-based materials. Due to the relative complexity of defining the shape of a CDCB specimen, MadabhusiRaman (1995) and Davalos, Madabhusi-Raman and Qiao (1997) proposed a numerical method based on the Rayleigh-Ritz solution to design the shape of the CDCB specimen. The basic idea of their work is to model the CDCB specimen as a cantilever beam to obtain its compliance for a given crack length. A method based on Rayleigh-Ritz solution is used to derive the expression of the compliance for a CDCB specimen, which is defined as a function of the crack length and the slope of the contour portions. For each discrete crack length, a linear shape of the contour is assumed. The expression for compliance rate-change $d C / d a$ is obtained by differentiating the expression for the compliance $C$ with respect to the crack length $a$.

Shear deformation is very important for FRP composites and wood materials. To account for shear effects, Madabhusi-Raman (1995) and Davalos et al. (1997) used a first- 
order shear deformation theory in their formulation. Generally, the contour portions of a CDCB specimen are made of the same material, while the adherends are made of different materials. The different materials for adherends lead to the different mechanical properties for each half of a CDCB specimen, particularly stiffness and compliance. In order to achieve the same compliance rate-change for each half of the specimen, the contour shape on each side of the CDCB specimen has to be designed separately, in order to account for dissimilar materials at the interface.

The methodology used previously at WVU for the design of the contour of a CDCB specimen is described next. For convenience, the geometric parameters used in defining one half of a CDCB specimen are represented graphically in Fig. 1.4.

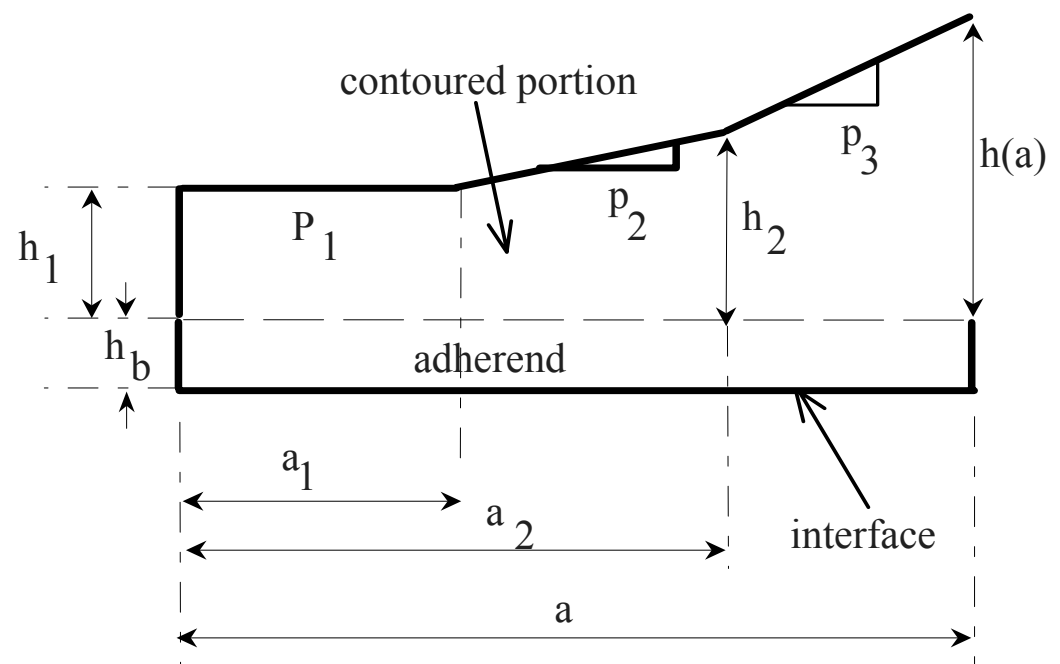

Fig. 1.4 Geometry of one half of a CDCB specimen from Madabhusi-Raman (1995) 
The following quantities are defined as, $h_{b}=$ constant height of the base adherend, $h_{l}=$ initial constant height of the bonded contour portion for an initial crack length $a_{1}, P_{l}=$ slope of the contoured portion in the range of $a_{1}$ (zero in this case). The rest of parameters, $h_{2}, a_{2}, P_{2}$, and so on have similar meanings as previous parameters. The importance of an initial constant height for the contoured part of a CDCB specimen is to provide sufficient material to accommodate loading fixtures, such as an attached hinge or a pin through the specimen. A step-by-step procedure for the design of the contoured shape of a CDCB specimen is summarized as:

1) Compute the compliance of the CDCB specimen by the Rayleigh Ritz method (Davalos, Madabhusi-Raman and Qiao 1997) as a function of crack length $a$ and slope $p$ : $C=C(a, p)$.

2) Differentiate the compliance function to obtain $d C / d a$.

3) Evaluate $d C / d a$ for a discrete value of $a$, say $a=a_{2}$ for $a_{2}>a_{1}$ (see Fig. 1.4).

4) Equate $d C / d a$, evaluated at a discrete crack-length, to a predefined constant value $K$ to solve for the slope, say $p=p_{2}$. That is, let $\frac{d C}{d a}\left(a_{2}, p_{2}\right)=K$ and solve for $p_{2}$.

5) Then, $h(a)=h_{1}+p_{2}\left(a-a_{1}\right)$ for $a>a_{1}$, say $h_{2}=h_{1}+p_{2}\left(a_{2}-a_{1}\right)$.

6) Repeat steps 1 through 5 to solve for $p_{3}$. 
This procedure is repeated for each incremental crack-length until the contour for the entire length of the CDCB specimen is obtained. The contour of the specimen is fitted by a continuous polynomial function through the discrete heights. The polynomial contour, called "exact" contour, is obtained from the above procedure has a convex shape. Accurately manufacturing such a complex geometry can be time consuming and costly, since it is necessary to test several specimens to obtain a good estimate of the fracture toughness of the interface. In order to reduce the effort and cost involved in manufacturing contoured parts of a CDCB specimen, the exact contour was simplified by a linear shape obtained through a regression analysis of the discrete heights at one-inch increments. The WVU investigators presented follow up studies of the simplified linear contour through analytical investigations of the compliance rate-change (Rayleigh Ritz method and finite element analysis), including an experimental program to verify the linear relationship of compliance versus crack length for linear-slope CDCB specimens.

The formulations involved in the Rayleigh-Ritz solution to derive the expression for the compliance of the CDCB specimen are described in detail by Madabhusi-Raman (1995), Davalos, Madabhusi-Raman and Qiao (1997) and Trimble (1999). However, there are a few minor errors in their original work. Since all the specimens for fatigue tests reported in the present study are designed based on these formulas, the significance of this information was the motivation for presenting again the corrected formulation for the CDCB specimen. 
The total strain energy of a beam element is given by

$$
U=\frac{1}{2} \int_{V} \sigma_{i j} \varepsilon_{i j} d V
$$

where, $\sigma_{i j}, \varepsilon_{i j}$ are the stresses and strains, respectively, and $V$ is the volume of the element. The kinematics of the beam including shear deformation is given by

$$
\begin{gathered}
u=-y \phi_{x}(x) \\
v=v(x)
\end{gathered}
$$

where, $\phi_{x}$ is the rotation of the normal to the mid-plane about the $z$ axis, and $v$ is the displacement in the $y$ coordinate. The strain-displacement relations resulting from the kinematics assumed above are

$$
\begin{aligned}
& \varepsilon_{x}=-y \phi_{x, x} \\
& \gamma_{x y}=v,_{x}-\phi_{x}
\end{aligned}
$$

where, a comma denotes differentiation with respect to the variable following it. The stress-strain relations are defined by

$$
\begin{gathered}
\sigma_{x}=E \varepsilon_{x} \\
\sigma_{x y}=G \gamma_{x y}
\end{gathered}
$$


Substituting equations (1.35) and (1.36) into equation (1.33), the following expression is obtained:

$$
\begin{gathered}
U=\frac{1}{2} \int_{0}^{a} \int_{A} E y^{2} \phi_{x, x}{ }^{2}+G\left(v, x_{x}-\phi_{x}\right)^{2} d x d A \\
=\frac{1}{2} \int_{0}^{a}\left[\left(\int_{A} E y^{2} d A\right) \phi_{x, x}{ }^{2}+\left(\int_{A} G d A\right)\left(v,_{x}-\phi_{x}\right)^{2}\right] d x \\
=\frac{1}{2} \int_{0}^{a}\left[E I \phi_{x, x}{ }^{2}+\kappa G A\left(v_{,_{x}}-\phi_{x}\right)^{2}\right] d x \\
=\frac{1}{2} \int_{0}^{a}\left[D(x) \phi_{x, x}{ }^{2}+F(x)\left(v,_{x}-\phi_{x}\right)^{2}\right] d x
\end{gathered}
$$

where, $I$ is the moment of inertia of the cross section, $A$ is the area of the cross section, $\kappa$ is the transverse shear correction factor, and $a$ represents the total cracked length of the CDCB specimen (length of the cantilever portion). $D(x)=E I$ and $F(x)=\kappa G A$, are the bending and shear stiffness of the CDCB specimen, respectively.

To use the CDCB specimen for interface bonds of dissimilar adherends, it is convenient to maintain a constant thickness of the adherends and to attach them to contoured portions made of a material that is easy to shape, such as wood. This approach can simplify the fabrication of the CDCB specimen. The following describes the procedure to determine the effective beam bending $(D)$ and shear stiffness $(F)$ for one-half of the CDCB specimen, where an adherend of constant cross section is attached to a contoured portion made of a different material. The concept of a transformed cross section is used to 
obtain the effective bending and shear stiffness of the CDCB specimen consisting of a twolayer laminate (Figure 1.5a), where the adherend is converted to an equivalent contour material (Figure 1.5b). The terms $D(x)=E I$ and $F(x)=\kappa G A$ that respectively represent the beam bending and shear stiffness in equation (1.37) are expressed as

$$
D(x)=b \sum E_{i}\left(t_{i} \bar{y}_{i}^{2}+\frac{t_{i}^{3}}{12}\right) \quad ; \quad F(x)=b \kappa \sum G_{i} t_{i} i=1,2
$$

where, $E_{i}$ and $G_{i}$ are the elastic and shear moduli of the $i$ th layer, $t_{i}$ is the thickness of the $i$ th layer of the cross section $\left(t_{l}=h_{c}\right.$ and $t_{2}=h_{b}$, Figure 1.5), and $\bar{y}_{i}$ is the distance of the centroidal axis of the $i$ th layer from the neutral axis of the cross section $\left(\bar{y}_{1}=\bar{y}_{c}\right.$ and $\bar{y}_{2}=$ $\bar{y}_{b}$, Figure 1.5). The neutral axis of bending is defined by eliminating the bendingextension coupling coefficient $(B=0)$. For convenience in this study, the neutral axis is defined in terms of the transformed section (Fig. 1.5b) as

$$
y_{n a}=\frac{m h_{b}{ }^{2}+h_{c}{ }^{2}+2 h_{b} h_{c}}{2\left(h_{c}+m h_{b}\right)}
$$

where, $m=E_{b} / E_{c}$, and the subscripts $c$ and $b$ refer respectively to the contour and base adherend of the CDCB specimen. The expressions for $\bar{y}_{i}$ of the layers are given by

$$
\bar{y}_{1}=\bar{y}_{c}=\frac{m\left(h_{b}{ }^{2}+h_{b} h_{c}\right)}{2\left(h_{c}+m h_{b}\right)} ; \bar{y}_{2}=\bar{y}_{b}=\frac{h_{c}{ }^{2}+h_{b} h_{c}}{2\left(h_{c}+m h_{b}\right)}
$$




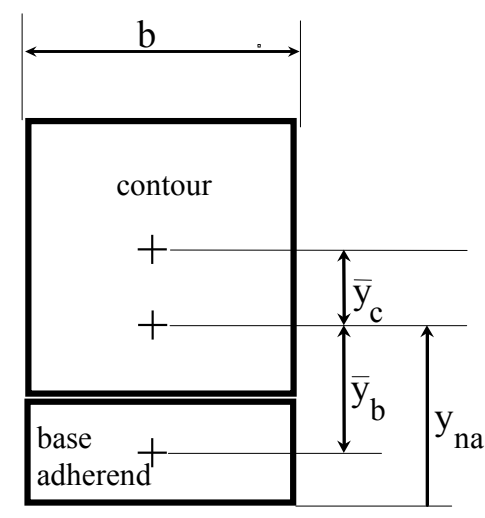

Fig. 1.5a Actual cross section

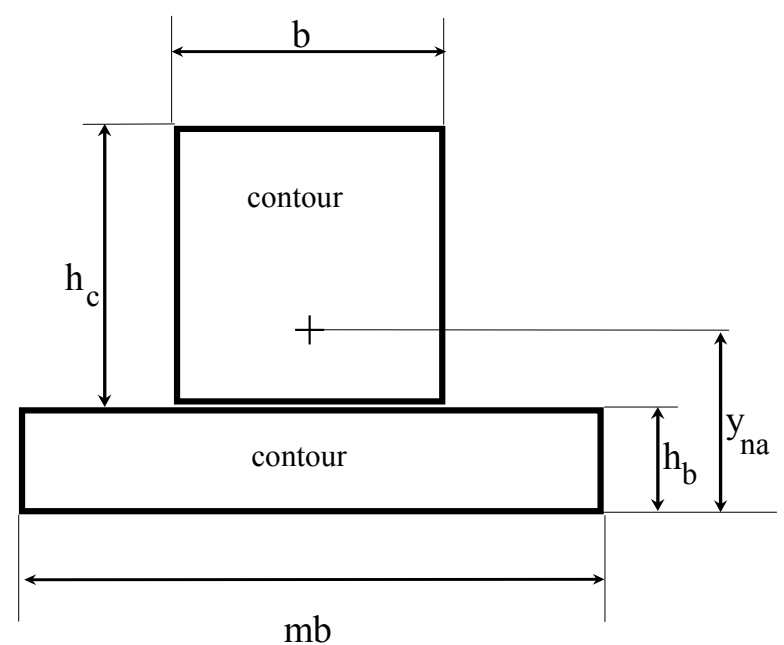

Fig. $1.5 \mathrm{~b}$ Transformed cross section

Fig. 1.5 Transformed cross section of a CDCB specimen

Substituting equation (1.40) into equation (1.38) and simplifying, the stiffness is defined as

$$
\begin{aligned}
& D(x)=E I=\frac{b E_{c}}{12}\left[\frac{3 m h_{c} h_{b}\left(h_{c}+h_{b}\right)^{2}}{h_{c}+m h_{b}}+h_{c}{ }^{3}+m h_{b}{ }^{3}\right] \\
& F(x)=\kappa G A=b \kappa\left(G_{b} h_{b}+G_{c} h_{c}\right)
\end{aligned}
$$

Assuming a linear contour shape,

$$
\begin{aligned}
& h_{c}=\left(h_{o}+k x\right)=h_{c}(x) \\
& h_{b}=\text { constant }
\end{aligned}
$$


where $h_{0}$ is the initial constant height and $k$ is the slope of the linear tapered specimen. From equation (1.41), the beam bending and shear stiffness is expressed as functions of $x$ and $k$ :

$$
\begin{aligned}
& D(x, k)=\frac{b E c}{12}\left[\frac{3 m\left(h_{o}+k x\right) h_{b}\left(h_{o}+k x+h_{b}\right)^{2}}{h_{o}+k x+m h_{b}}+\left(h_{o}+k x\right)^{3}+m h_{b}{ }^{3}\right] \\
& F(x, k)=b K\left[G_{b} h_{b}+G_{c}\left(h_{o}+k x\right)\right]
\end{aligned}
$$

The Rayleigh Ritz solution requires a representation of the variation of the displacement and rotational degrees of freedom by interpolation functions over the entire domain of the system. Hence, the displacement and rotational degrees of freedom are approximated as

$$
\begin{gathered}
v(x)=v_{i} N_{i}(x) \\
\phi_{x}(x)=\phi_{i} \quad M_{i}(x) \quad i=1,2, . . n
\end{gathered}
$$

where, $n$ is chosen to achieve a desired degree of accuracy, $v_{\mathrm{i}}$ and $\phi_{i}$ are the unknown coefficients of the variables, and $N_{\mathrm{i}}(x)$ and $M_{\mathrm{i}}(x)$ are the interpolation functions satisfying the essential boundary conditions, namely $v(a)=\phi_{x}(a)=0$. Algebraic functions satisfying these boundary conditions are given by

$$
N_{i}(x)=M_{i}(x)=(a-x)^{i}
$$


Substituting equations (1.43) and (1.44) in equation (1.37), the strain energy can be written as

$$
\begin{gathered}
U=\frac{1}{2} \int_{0}^{a} D(x, k)\left(\phi_{i} M_{i, x} \phi_{j} M_{j, x}\right) d x \\
+\frac{1}{2} \int_{0}^{a} F(x, k)\left(v_{i} N_{i, x}-\phi_{i} M_{i, x}\right)\left(v_{j} N_{j, x}-\phi_{j} M_{j, x}\right) d x \\
i, j=1,2, . . n
\end{gathered}
$$

Equation (1.46) is rearranged in matrix form and is expressed as

$$
\begin{gathered}
U=\frac{1}{2} \int_{0}^{a} D(x, k)\left[\phi_{i}\right]\left[M_{i, x} M_{j, x}\right]\left\{\phi_{j}\right\} d x \\
+\frac{1}{2} \int_{0}^{a} F(x, k)\left[v_{i}\right]\left[N_{i, x} N_{j, x}\right]\left\{v_{j}\right\} d x \\
-\int_{0}^{a} F(x, k)\left[v_{i}\right]\left[N_{i, x} M_{j}\right]\left\{\phi_{j}\right\} d x \\
+\frac{1}{2} \int_{0}^{a} F(x, k)\left[\phi_{i}\right]\left[M_{i} M_{j}\right]\left\{\phi_{j}\right\} d x \\
i, j=1,2, . . n
\end{gathered}
$$

After grouping similar terms, equation (1.47) is written in a concise form as

$$
\begin{gathered}
U=\frac{1}{2}\left[\phi_{i}\right]\left[\left[A_{1}\right]+\left[A_{3}\right]\right]\left\{\phi_{j}\right\}+\frac{1}{2}\left[v_{i}\right]\left[A_{4}\right]\left\{v_{j}\right\}-\left[v_{i}\right]\left[A_{2}\right]\left\{\phi_{j}\right\} \\
i, j=1,2, . . n
\end{gathered}
$$

where 


$$
\begin{aligned}
& A_{l i j}=\int_{0}^{a} D(x, k) M_{i, x} M_{j, x} d x \\
& A_{2 i j}=\int_{0}^{a} F(x, k) N_{i, x} M_{j} d x \\
& A_{3 i j}=\int_{0}^{a} F(x, k) M_{i} M_{j} d x \\
& A_{4 i j}=\int_{0}^{a} F(x, k) N_{i, x} N_{j, x} d x \\
& i, j=1,2, . . n
\end{aligned}
$$

The work done by the applied concentrated tip-load at $x=0$ is given by

$$
\begin{gathered}
W=P v(0) \\
=\left[v_{i}\right]\{R\}, \text { where }\{R\}=P\left\{N_{i}(0)\right\} \quad i=1,2, . . n
\end{gathered}
$$

The total potential energy can be expressed using equations (1.48) and (1.49) as

$$
\begin{gathered}
\Pi=\frac{1}{2}\left[\phi_{i}\right]\left[\left[A_{1}\right]+\left[A_{3}\right]\right]\left\{\phi_{j}\right\}+\frac{1}{2}\left[v_{i}\right]\left[A_{4}\right]\left\{v_{j}\right\}-\left[v_{i}\right]\left[A_{2}\right]\left\{\phi_{j}\right\}-\left[v_{i}\right]\{R\} \\
i, j=1,2, . . n
\end{gathered}
$$

The minimization of the total potential energy with respect to the unknown displacement vectors yields the expressions

$$
\begin{gathered}
\frac{\partial \prod}{\partial\left[v_{i}\right]}=\left[A_{4}\right]\left\{v_{j}\right\}-\left[A_{2}\right]\left\{\phi_{j}\right\}-\{R\}=0 \\
\frac{\partial \prod}{\partial\left[\phi_{i}\right]}=-\left[A_{2}\right]^{T}\left\{v_{j}\right\}+\left[\left[A_{1}\right]+\left[A_{3}\right]\right]\left\{\phi_{j}\right\}=0
\end{gathered}
$$


From equation (1.51), the variables $\left\{v_{i}\right\}$ and $\left\{\phi_{i}\right\}$ are determined by solving the following simultaneous equations:

$$
\left\{\begin{array}{l}
\left\{v_{i}\right\} \\
\left\{\phi_{i}\right\}
\end{array}\right\}=\left[\begin{array}{rr}
{\left[A_{4}\right]} & -\left[A_{2}\right] \\
-\left[A_{2}\right]^{T} & \left.\left[A_{1}\right]+\left[A_{3}\right]\right]
\end{array}\right]^{-1}\left\{\begin{array}{r}
\{R\} \\
\{0\}
\end{array}\right\}
$$

The solution of equation (1.51) yields the displacement parameter $\left\{v_{i}\right\}$, which is substituted in equation (1.44) to evaluate the displacement function $v(x)$. Finally, the compliance is obtained by computing $v(x)$ at $x=0$, for a unit tip-load.

For a linear tapered specimen loaded with a unit tip-load, the compliance is expressed as

$$
C=v(0)=f_{C}(a, k)
$$

where, $k$ is the linear slope of the specimen, and $a$ is the length of cantilever beam. Further, the compliance rate change can be derived as

$$
\frac{d C}{d a}=\frac{d f_{c}(a, k)}{d a}
$$

Equations (1.53) and (1.54) are the expressions for compliance and compliance rate change, and they are functions of the crack length and slope of the cantilever beam. 
The above computational procedure involves symbolic mathematical operations, such as matrix inversion and differentiation, and therefore, the mathematics symbolic manipulator MAPLE is used to obtain the solution. The algorithms of the MAPLE programs can be found in the theses by Madabhusi-Raman (1995) and Trimble (1999).

The design of an exact contoured specimen is a time-consuming process and involves several computational steps. Trimble (1999) introduced a modified method to simplify the above design approach as a two-step method:

1. From Eq. (1.54), for a given constant $d C / d a$, compute the slope $k$ for various crack lengths.

2. Linearize the set of slopes obtained in step 1 , and define it as the specimen global slope. Further, the $d C / d a$ used in step 1 becomes the specimen compliance rate change.

The CDCB specimen designed by the above procedure can be efficiently used to evaluate the fracture of hybrid interfaces (see Davalos, Qiao and Trimble, 2000a and 2000b). This simplified procedure was used in the present study to design the CDCB specimen for fatigue tests. 


\section{CHAPTER 2}

\section{EXPERIMENTAL WORK}

\subsection{Introduction}

A crack can experience three kinds of loading: (1) Mode-I loading, (2) Mode-II loading, and (3) mode-III loading (Anderson 1994), as shown in Fig. 2.1.

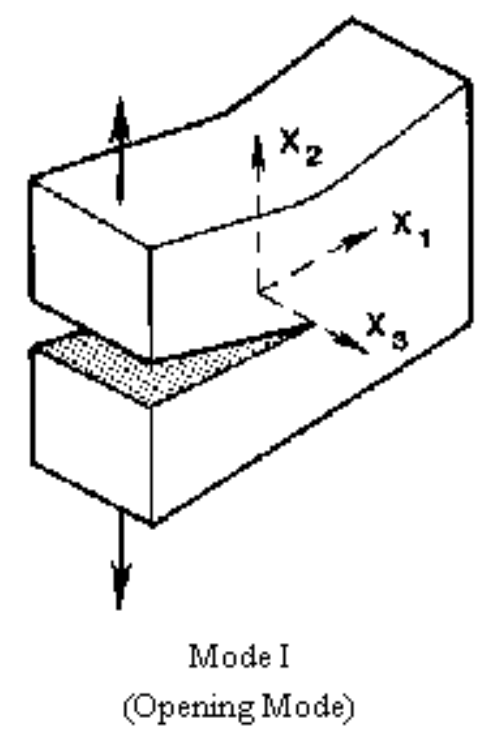

(a) Schematic illustration of Mode-I loading 


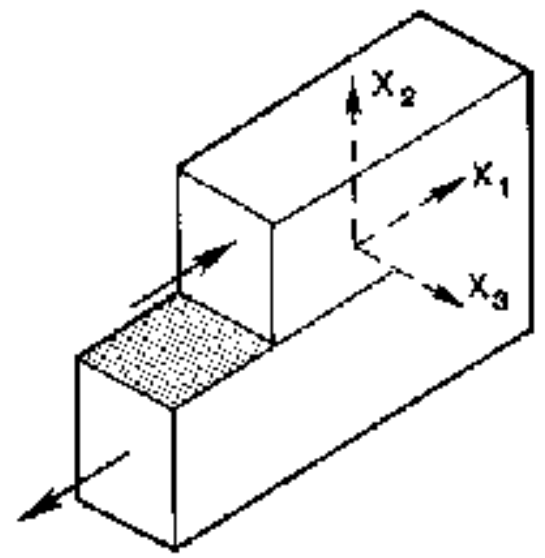

Mode II

(Slidirg Mode)

(b) Schematic illustration of Mode-II loading

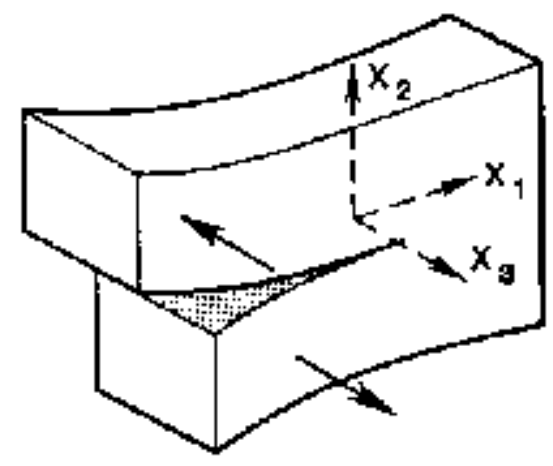

\section{Mode III}

(Tearing Mode)

(c) Schematic illustration of Mode-III loading

Fig. 2.1 Modes of loading that can be applied to a crack (From Anderson 1994) 
Mode-I loading is also called opening loading and tends to open the crack. The applied load is normal to the crack (see Fig. 2.1 a). Mode-II or sliding mode loading tends to slide one crack face with respect to the other, and it corresponds to in-plane shear loading (see Fig. 2.1 b). Mode-III loading refers to out-of-plane shear loading (see Fig. 2.1 c). It has been pointed out by Anderson (1994) that a cracked element could be subjected to any one of the above three modes of loading, or a combination of two or three of these modes.

In this study we only consider the Mode-I fatigue loading for interfaces of phenolic FRP composite bonded to red maple wood. It has been shown that the delamination of the bonded interface for these materials can be explained by linear fracture mechanics models (Davalos, Madabhusi-Raman and Qiao 1997; and Trimble 1999). The bonded interface for these materials is generally less resistant to crack extension under opening load than under in-plane shear or out-of-plane shear load. Recently, the strain energy release rate for ModeII was found to be more than double the value reported for Mode-I, for the same bonded materials (Qiao, Wang and Davalos 2002).

A linear-shape contoured double cantilever beam (CDCB) specimen was used for the fatigue tests of phenolic FRP-red maple wood bonded interface. The CDCB specimen was designed by the Rayleigh-Ritz method (Madabhusi-Raman 1995 and Trimble 1999). This specimen provided constant crack growth rate within the second stage of fatigue process when subjected to constant cyclic loading conditions. The adherends of the CDCB specimen consisted of red maple wood and pultruded phenolic FRP, and the contoured 
portions of the specimen were made of laminated veneer lumber (LVL). A significant amount of work has been accomplished at WVU on performance of FRP-wood bonded interfaces under static and environmental loading conditions, but no information was available until the present study on the fatigue behavior of bonded interfaces.

This chapter presents information on materials, test specimen and testing procedures.

\subsection{Materials}

The wood material used in the present study is the hardwood species red maple, and the reinforcing material is a phenolic FRP composite. The phenolic FRP composite material is produced by the pultrusion process, a well-controlled and relatively low cost manufacturing process that is used in the production of FRP composites. The lay-up of the FRP laminate consists of two continuous strand mat (CSM) layers and one unidirectional roving layer. The CSM layers provide a desirable surface texture for obtaining a good adhesive bond interface. The fiber weight and volume fractions of the phenolic FRP composite are $70.05 \%$ and $50.98 \%$ respectively. A CDCB specimen is used to study the fracture toughness of the interface of phenolic FRP composite bonded to red maple wood. Both contoured parts of the CDCB specimen are made of laminated veneer lumber (LVL), which is a wood composite material easy to shape. The wood adhesive Resorcinol Formaldehyde (RF, Penacolite G-1131) is used to bond the phenolic FRP composite to red 
maple wood. As concluded from the modified ASTM D 2559 testing results by Trimble (1999), there is no need to prime the wood surface for improved bond strength; therefore, no bonding coupling agent was used in this study. The suppliers of the RF adhesive and materials for the CDCB specimen are listed in Table 2.1 for reference.

Table 2.1 Experimental materials and their sources

\begin{tabular}{cc}
\hline Materials & Suppliers \\
\hline $\begin{array}{c}\text { Resorcinol-Formaldehyde } \\
\text { adhesive } \\
\text { Phenolic FRP composite } \\
\text { Laminated veneer lumber }\end{array}$ & Indspec Chemical Corp., Pittsburgh, PA, USA \\
Truss Joist MacMillen (TJM), Buckhannon, WV, USA \\
Red maple wood \\
Wood Industries, West Virginia University, \\
Morgantown, WV, USA
\end{tabular}




\subsubsection{Red maple}

Selected red maple (Acer rubrum) samples of furniture quality, with no visible defects, are used as the wood adherend to be bonded to the FRP composite. The final geometry of the red maple strip for bonding to the FRP is 1.25 " wide x 12 " long x 0.1875 " thick. The red maple surface was planed and then cleaned by using compressed air before bonding.

\subsubsection{Laminated veneer lumber (LVL)}

Yellow poplar (Liriodendron tulipifera) laminated veneer lumber (LVL) is used as the contoured portion of the CDCB specimen. The low variation of the density of LVL material makes it suitable for the contoured part of the CDCB specimen. The LVL lay-up consists of 15 layers of yellow poplar, each 1/8 inch thick (see Figure 2.2). The dimensions of the rectangular LVL samples for bonding to the wood and FRP composite strips were 2.125 " wide x 13.5 " long x 1.25 " thick. 


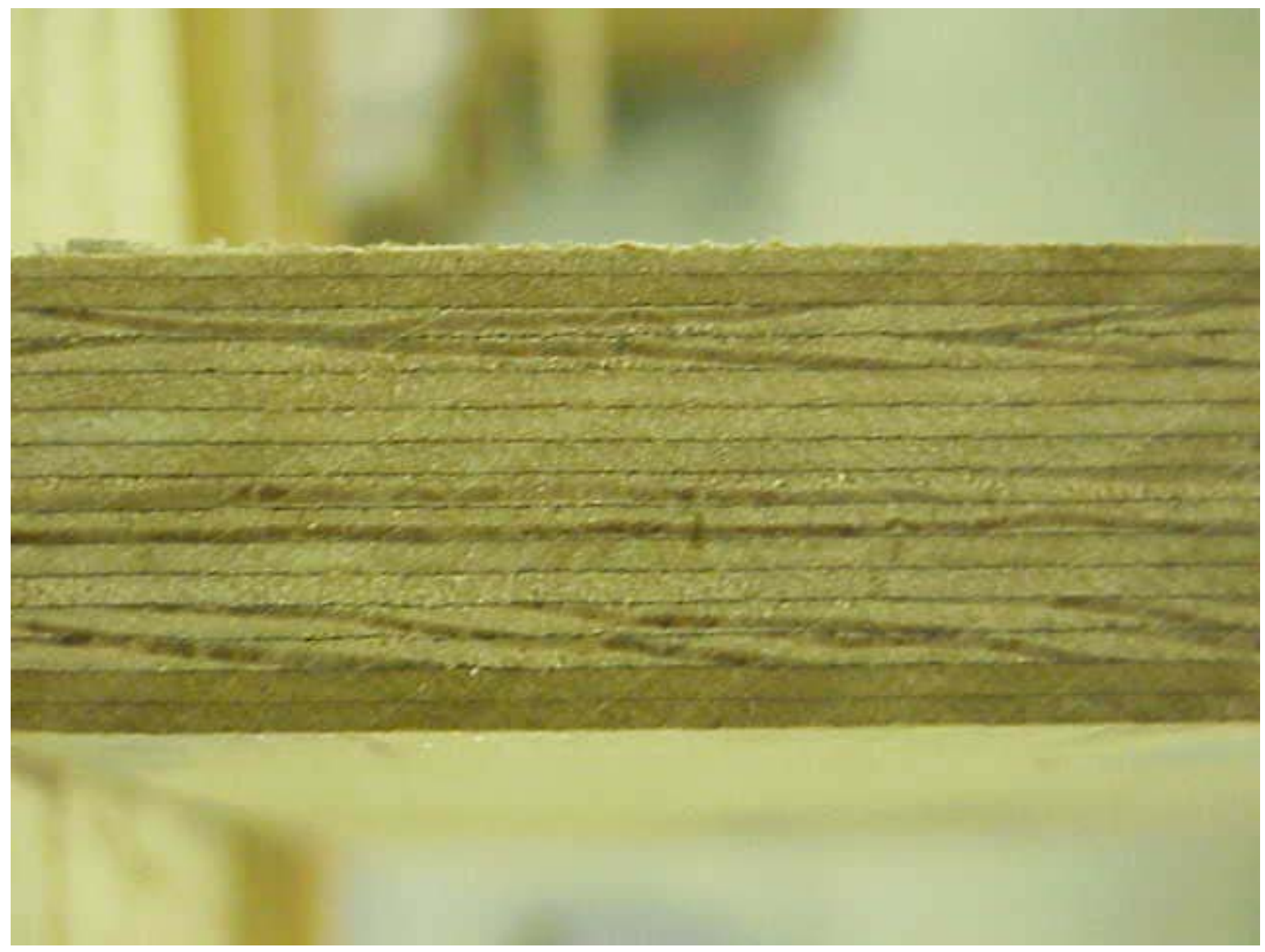

Fig. 2.2 Lay-up of the yellow poplar laminated veneer lumber (LVL) 


\subsubsection{Phenolic FRP composite}

Phenolic fiber-reinforced polymer (FRP) composite material is used to reinforce the red maple wood material. The phenolic FRP composite is produced by the pultrusion process. Pultrusion is a continuous manufacturing process used to produce constant crosssection shapes of any length. It is a low-cost process because of the achievement of direct conversion from continuous fibers and resin into a finished product. In the pultrusion process, the fibers are continuously impregnated and pulled through a heated die, where they are shaped and cured. Operational costs of pultrusion are low. The production equipment, such as chrome-plated dies and design and tune-up of the guiding system, is the major cost item of pultrusion. Therefore, pultrusion is ideally suited for high volume productions (Barbero 1998).

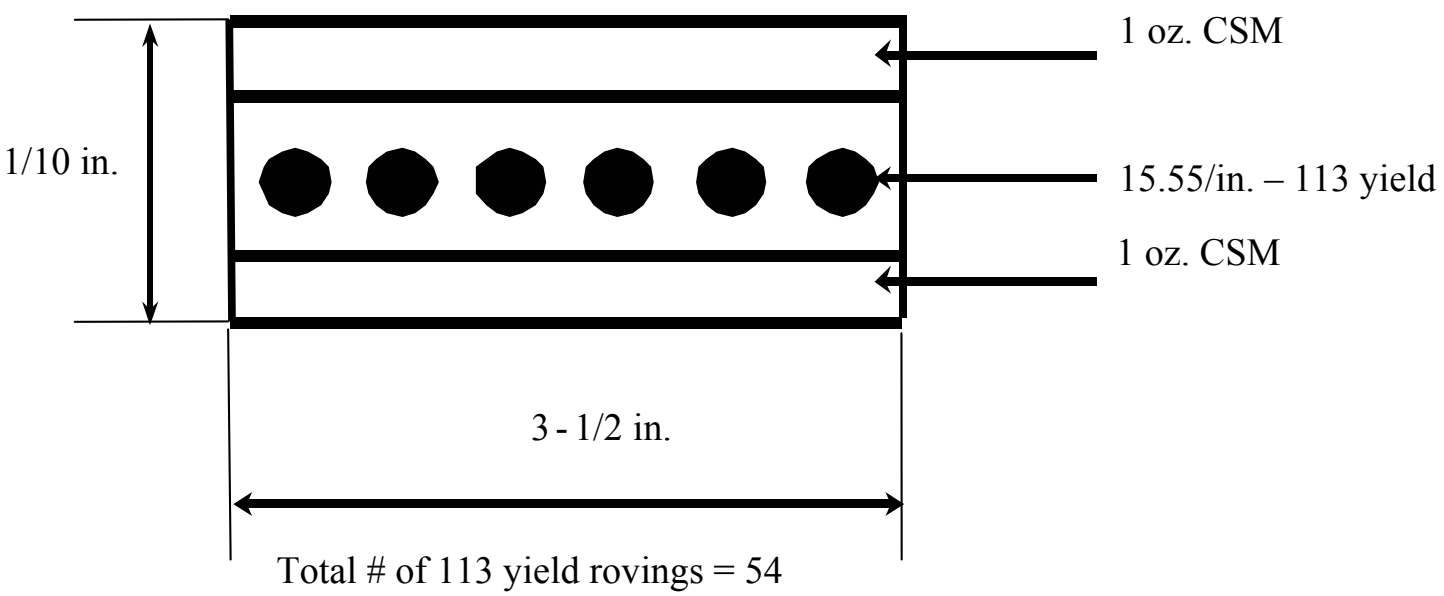

Fiber Weight Fraction $=70.05 \%$; Fiber Volume Fraction $=50.98 \%$

Fig. 2.3 Material architecture of the phenolic FRP strip 
The phenolic FRP composite selected for this study consists of E-glass fiber rovings embedded in a phenolic resin matrix. Both top and bottom surfaces of the phenolic FRP composite contained continuous strand mat (CSM) layers. The CSM layer has a desirable surface texture, which is very helpful to obtain a good adhesive bond between the phenolic FRP composite and red maple wood. The lay-up of the phenolic FRP laminate is shown in Figure 2.3. The phenolic FRP surfaces are moderately sanded with a 3M product 100 grain sandpaper, then cleaned by compressed air, and then wiped with a $91 \%$ Isopropyl alcohol, before bonding. The final geometry of the phenolic FRP composite for bonding to wood is 1.25 " wide x 12 " long x 0.10 " thick.

\subsubsection{Adhesive}

Resorcinol formaldehyde (RF: Pencolite G-1131) adhesive from Indspec Chemical Corporation is used for bonding the phenolic FRP to red maple wood. The RF adhesive consists of two components: resorcinol formaldehyde polymer (G-1131-A) and paraformaldehyde mixture (G-1131-B). Resorcinol formaldehyde polymer (G-1131-A) is a brown color liquid resin and paraformaldehyde mixture (G-1131-B) is a white powder hardener. Resorcinol formaldehyde (RF) adhesive is made from mixing the resorcinol formaldehyde polymer (G-1131-A) and paraformaldehyde (G-1131-B) by weight ratio of five to one. The RF adhesive is neutral and inert, and it provides resistance to deterioration or disintegration due to aging and wetting/drying cycles. Trimble (1999) used the same resorcinol formaldehyde (RF) and phenol-modified resorcinol formaldehyde (PRF) adhesives to study the bond strength of the phenolic FRP-red maple wood interface with 
and without coupling agents (primers). Two kinds of primers, diluted resorcinol formaldehyde (RF) and hydroxymethylated resorcinol (HMR), were tested in his study. He indicated that resorcinol formaldehyde (RF) adhesive was more suitable than phenolmodified resorcinol formaldehyde (PRF) adhesive for red maple wood bonding. His study also showed that phenolic FRP-wood bond line performed reasonably well without the need for coupling agent; therefore, only the RF adhesive without a coupling agent is used in this study to bond phenolic FRP-red maple wood interfaces.

\subsection{Mechanical Properties of Materials}

The mechanical properties of phenolic FRP composite, laminated veneer lumber (LVL) and red maple wood are needed to design the CDCB specimen. The modulus of elasticity (Young's modulus) and shear modulus of the red maple and LVL were obtained from tension and torsion tests, respectively (Trimble 1999). For tension tests, 12 specimens were conditioned to $12 \%$ moisture content in an environmental chamber. The dimensions of red maple and LVL test coupons were $1 "$ x $0.5 " \times 20 "$ and $1 " \times 0.375 " \times 20$ ", respectively. For the torsion tests, eight samples were prepared. The dimensions of red maple and LVL test coupons were $1.5 "$ x 1.5" x 17" and 1.5" x 1" x 17", respectively. Six phenolic FRP strips were tested under tension to obtain Young's modulus. The phenolic FRP strips were too flexible to be tested in torsion; therefore, the shear modulus predicted by a micro/macro-mechanics model (Davalos et al. 1996) was adopted in this study. The mechanical properties of phenolic FRP, LVL and red maple are summarized in Table 2.2. 
These material properties in Table 2.2 were used to design the phenolic FRP-red maple wood CDCB specimen.

Table 2.2 Mechanical properties of phenolic FRP composite, laminated veneer lumber (LVL) and red maple wood

\begin{tabular}{ccc}
\hline Materials & Young's modulus E, GPa & Shear modulus G, Gpa \\
\hline Phenolic FRP & $30.41^{\mathrm{a}}(\mathrm{COV}=0.55 \%)$ & $4.48^{\mathrm{b}}$ \\
Laminated veneer lumber & $11.72^{\mathrm{a}}(\mathrm{COV}=5.96 \%)$ & $0.69^{\mathrm{c}}(\mathrm{COV}=3.81 \%)$ \\
Red maple & $13.71^{\mathrm{a}}(\mathrm{COV}=4.57 \%)$ & $1.25^{\mathrm{c}}(\mathrm{COV}=5.40 \%)$ \\
\hline
\end{tabular}

${ }^{\mathrm{a}}$ Tension tests, ${ }^{\mathrm{b}}$ Micro/macro-mechanics model, ${ }^{\mathrm{c}}$ Torsion tests.

\subsection{Shape of the CDCB Specimen}

The contour shape of the CDCB specimen was designed using the Rayleigh-Ritz method. The CDCB specimen is contoured to achieve a constant rate of compliance change with respect to crack length, $d C / d a$. The CDCB specimen design and applications to woodFRP interface fracture studies are given in several papers by Davalos et al. $(1997,1998 \mathrm{a}$, 1998b, 2000a and 2000b) and Qiao et al. (2000). The compliance rate change $d C / d a$ for the linear slope CDCB specimen was predicted by the Rayleigh-Ritz method. Also a modified Rayleigh-Ritz method, in which higher order shape functions for the deflection and rotation were used to replace the quadratic shape functions used in the original Rayleigh- 
Ritz method, was further developed by Trimble in 1999 (see section 1.5.2). Based on the design procedure given in section 1.5.2 and the material properties described in section 2.3, the contour shape of the $\mathrm{CDCB}$ specimen was designed. The geometric details of the CDCB specimen are shown in Fig. 2.4, and the adherends consist of red maple wood and pultruded phenolic FRP, with the contoured portions made of laminated veneer lumber (LVL).

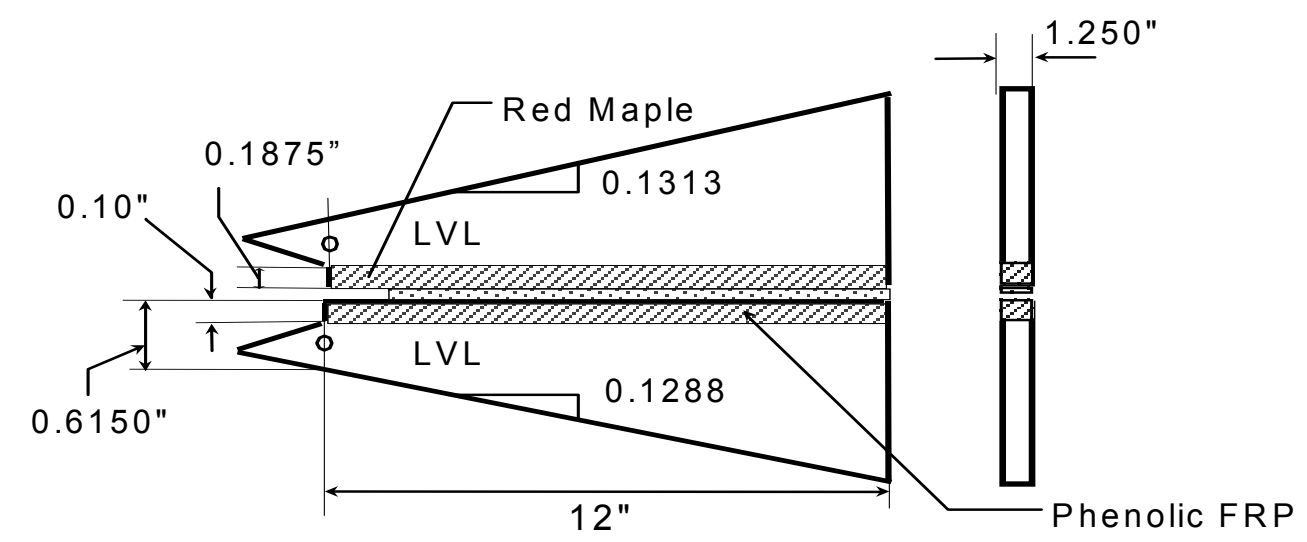

Fig. 2.4 Geometry of the CDCB specimen for fatigue tests

Compliance calibration tests were conducted for several red maple wood-phenolic FRP composite CDCB samples, and the average $d C / d a$ value obtained from experiments 
was $29.59 \times 10^{-5} \mathrm{lb}^{-1}$, and this value is used to calculate strain energy release rate $G$ in the present study.

The conventional equation for mode-I strain energy release rate is given as

$$
G=\frac{P^{2}}{2 b} \frac{d C}{d a}
$$

where $P$ is the applied load, $b$ is the thickness of the specimen, $d C / d a$ is a constant rate of compliance change with respect to crack length. For cyclic loading, the strain energy release rate range $\Delta G$ is defined as

$$
\Delta G=G_{\max }-G_{\min }
$$

where, $G_{\max }$ and $G_{\min }$ correspond to the maximum and minimum strain energy release rate in a loading cycle. They are obtained by substituting the maximum and minimum loads, respectively, into equation 2.1 and are expressed as

$$
\begin{gathered}
G_{\max }=\frac{P_{\max }^{2}}{2 b} \frac{d C}{d a} \\
G_{\min }=\frac{P_{\min }^{2}}{2 b} \frac{d C}{d a}
\end{gathered}
$$




\subsection{CDCB Specimen Preparation}

Based on the shape of the CDCB specimen designed in section 2.4, the CDCB specimens were manufactured. The combination of the interface materials is red maple wood and phenolic FRP composite. The procedure to fabricate a CDCB specimen is described in the following. Before bonding, all the bonding surfaces of the wood materials (red maple and LVL) were planed and of the phenolic FRP were sanded, and the dust removed by blowing compressed air. The surfaces of the phenolic FRP were further cleaned with 91\% Isopropyl alcohol. The cleaned phenolic FRP composite strip was marked with a permanent marker at three inches from one end, and a folded scotch tape insert was used to define a three-inch long pre-crack for a CDCB specimen. Sufficient amount of RF adhesive was mixed before bonding;; for example, 94.50 grams resorcinol formaldehyde polymer (G-1131-A) and 18.90 grams paraformaldehyde mixture (G-1131B) would be sufficient for bonding seven CDCB specimens. The two components of RF adhesive were placed into a plastic container and stirred completely with a glass stick. In order to avoid the CDCB specimens sticking together or bonding to the pressure clamps, wax paper was used to separate the specimens and the pressure clamps. To fabricate a CDCB specimen, first, the two adherends (red maple and phenolic FRP) were bonded together to form the actual interface. The RF adhesive was spread evenly onto the bonding surface of red maple and phenolic FRP composite strips with a painting brush. Since the interface bond is the key issue of concern in this study, one must be very careful when spreading the RF adhesives (either too much or too little RF adhesive can lead to bad testing results). Then, two pieces of rectangular blocks of LVL with dimensions of 2.125" 
wide x 13.5 " long x 1.25 " thick were each glued to each adherend (red maple and phenolic FRP).

The assembled CDCB specimens were clamped in a press to apply a specified pressure on the interface bonds. The side view and front view of the specimen assemblies are shown in Figs. 2.5 and 2.6. The nuts on the six alignment bolts are first tightened by hand. Then a digital torque wrench is used to continue tighten the nuts until the ideal pressure is reached. The optimum open and closed assembly time of 5 and 30 minutes and 210 psi pressure suggested by Trimble (1999) from his ASTM D 2559 test results were adopted in this study. The CDCB samples were required to cure for 24 hours before removing them from the clamps. Any excessive RF adhesive on both sides of the CDCB samples was removed by a scrapper. The contoured shape of the CDCB specimen was marked on one side of the bonded assembly, and a band saw was used to cut the LVL material in order to get the geometry of the CDCB specimen (Fig. 2.4). A hole of diameter $13 / 64$ " was drilled on each side at the tip of the contoured portion to insert the loading pin of the loading fixture. This completes the fabrication of the specimen for testing. 


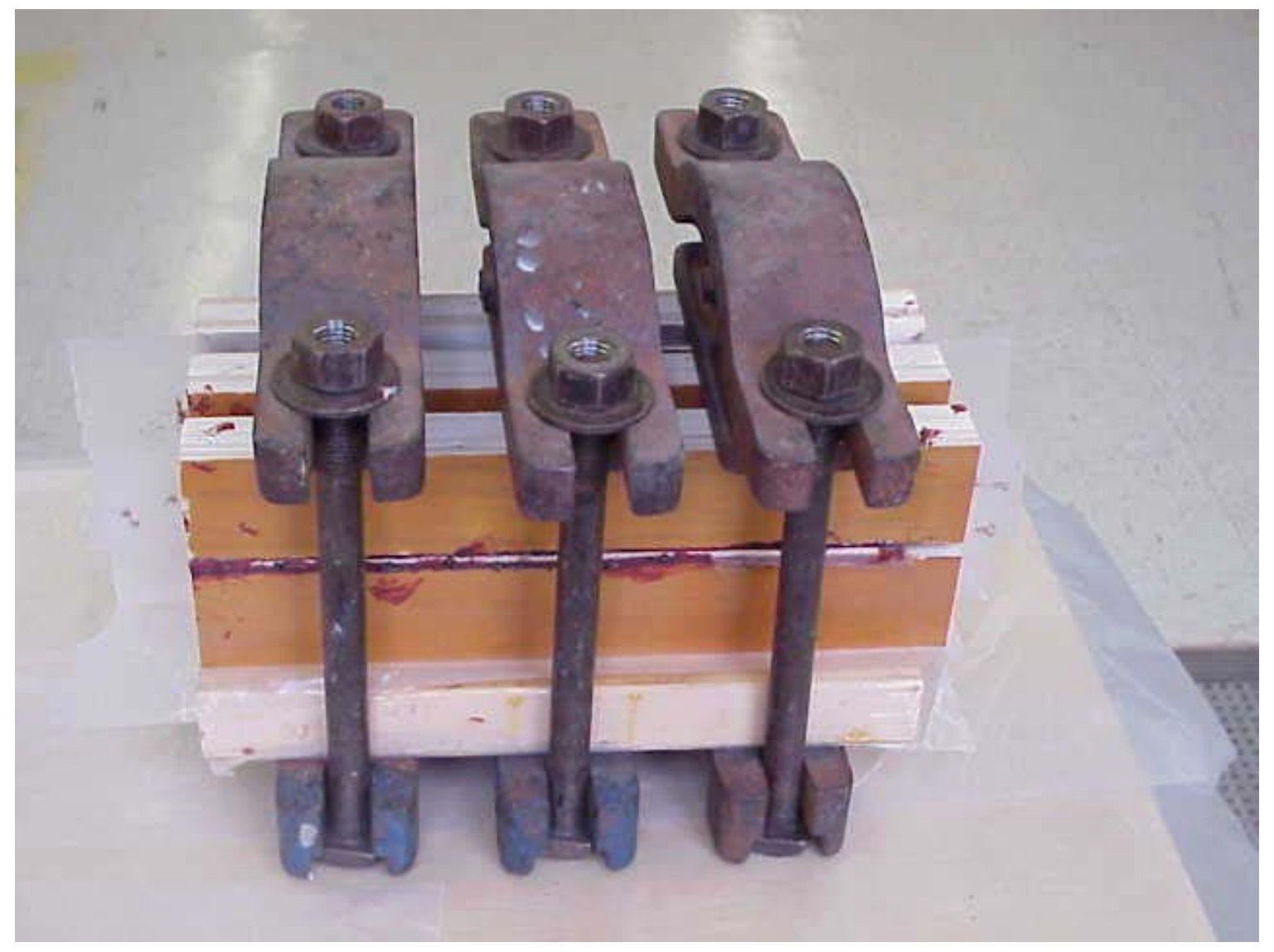

Figure 2.5 Side view of the CDCB specimen assembly under pressure for curing the interface bonds 


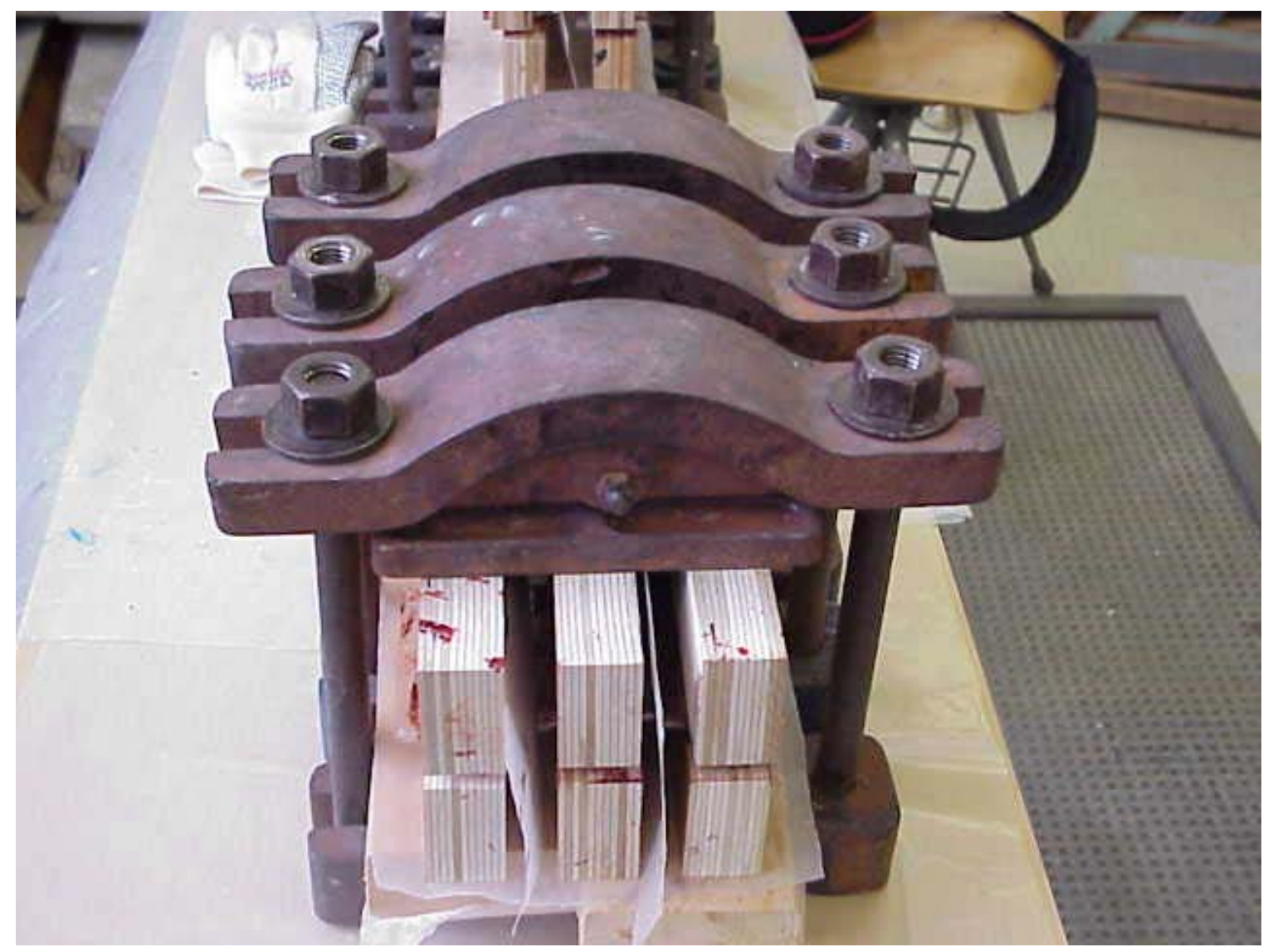

Figure 2.6 Front view of the CDCB specimen assembly under pressure for curing the interface bonds 


\subsection{Fatigue Test}

All tests are carried out at room temperature and laboratory ambient conditions. A schematic illustration of constant amplitude cyclic load with a sinusoidal waveform is given in Fig. 2.7, where load ratio (the ratio of minimum load to maximum load) is kept constant at a frequency of $1 \mathrm{~Hz}$. The experiment is performed under load control mode and the maximum value of a cyclic load is less than the critical static load. Mode-I fatigue tests are conducted in an MTS servo hydraulic testing machine (see Fig. 2.8). The tests were controlled by a computer through pre-programmed software called GPA. The cyclic load was applied by using a loading fixture made of aluminum. Fig. 2.9 (a) shows the front view and side view of the loading fixture. Before the testing, a small pin or nail is attached to the end of the specimen in order to support its weight vertically by a plastic thread connected to the upper frame of the testing machine (Fig. 2.14 b). A fixed cyclic load was applied to the CDCB specimen for each test, and the crack opening displacement (COD), as measured by the loading pin displacement, was plotted as a function of number of cycles. Fig. 10 shows one of the CDCB specimens under fatigue tests. The applied load $P_{\max }$ and $P_{\min }$ were used in Eqs. (2.3) and (2.4) to obtain the strain energy release rates $G_{\max }$ and $G_{\text {min }}$. The compliance method is used to measure crack propagation rate $d a / d N$, which is calculated as follows:

$$
\frac{d C}{d a}=k=\text { constant }
$$


Since compliance $C=\frac{\Delta}{P}$, at constant load $P$, where $\Delta$ is the displacement.

$$
\frac{d C}{d N}=\frac{1}{P} \frac{d \Delta}{d N}
$$

Applying the chain rule, we have

$$
\frac{d a}{d N}=\frac{d a}{d C} \frac{d C}{d N}
$$

From Eqs. (2.5) and (2.6), $\frac{d a}{d N}$ is obtained as

$$
\frac{d a}{d N}=\frac{1}{k P} \frac{d \Delta}{d N}
$$

As is generally done for fatigue crack propagation studies, the crack growth rate $d a / d N$ was plotted as a function of the strain energy release rate range $\Delta G$. 


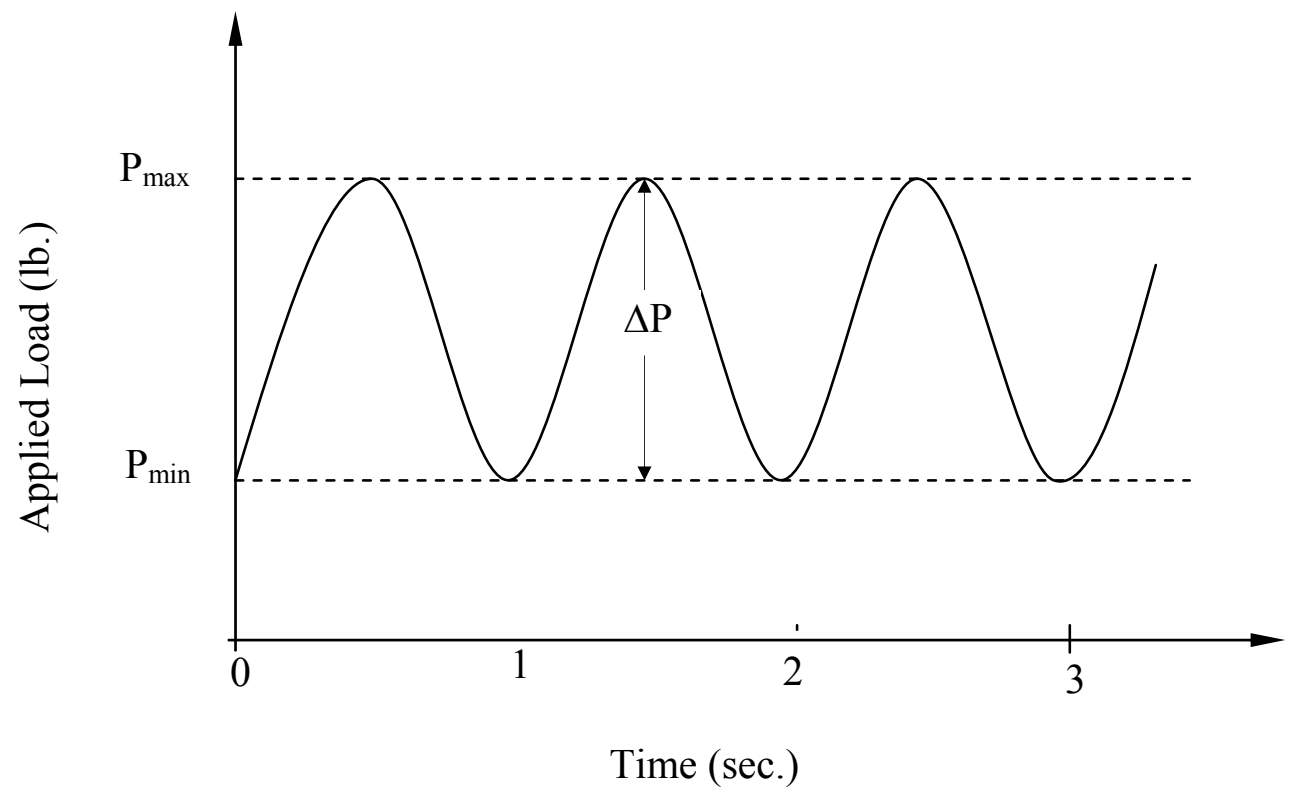

Figure 2.7 A schematic illustration of constant amplitude cyclic loading 


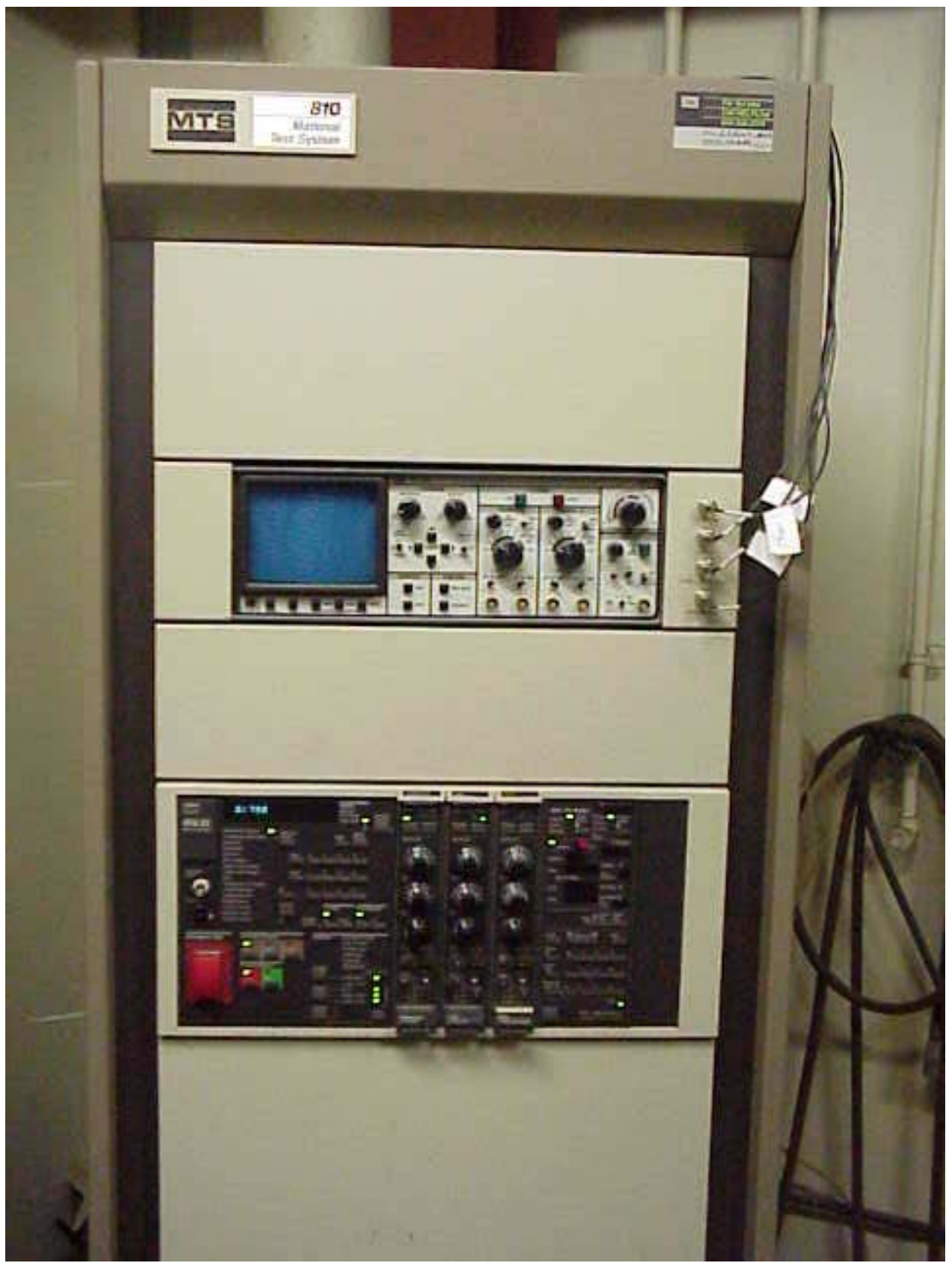

Figure 2.8 The MTS servo hydraulic testing machine for Mode-I fatigue tests 


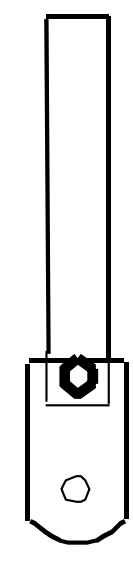

Side view

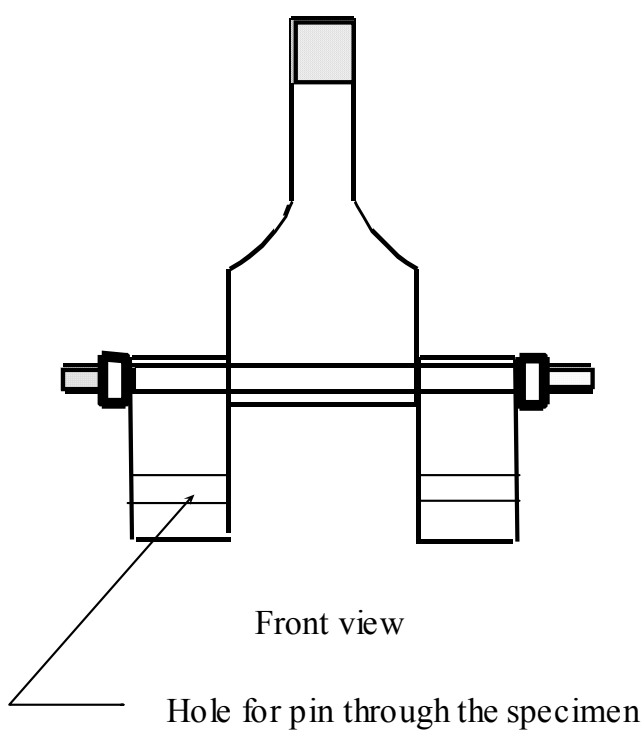

(a) Loading fixture for the CDCB specimen

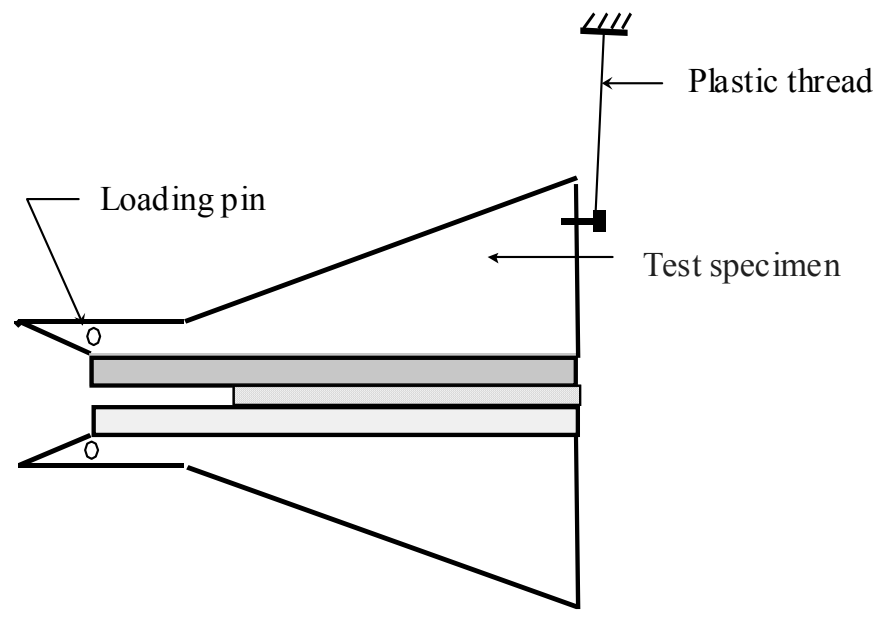

(b) Front view of CDCB specimen for loading

Figure 2.9 Experimental setup for mode-I fatigue tests 


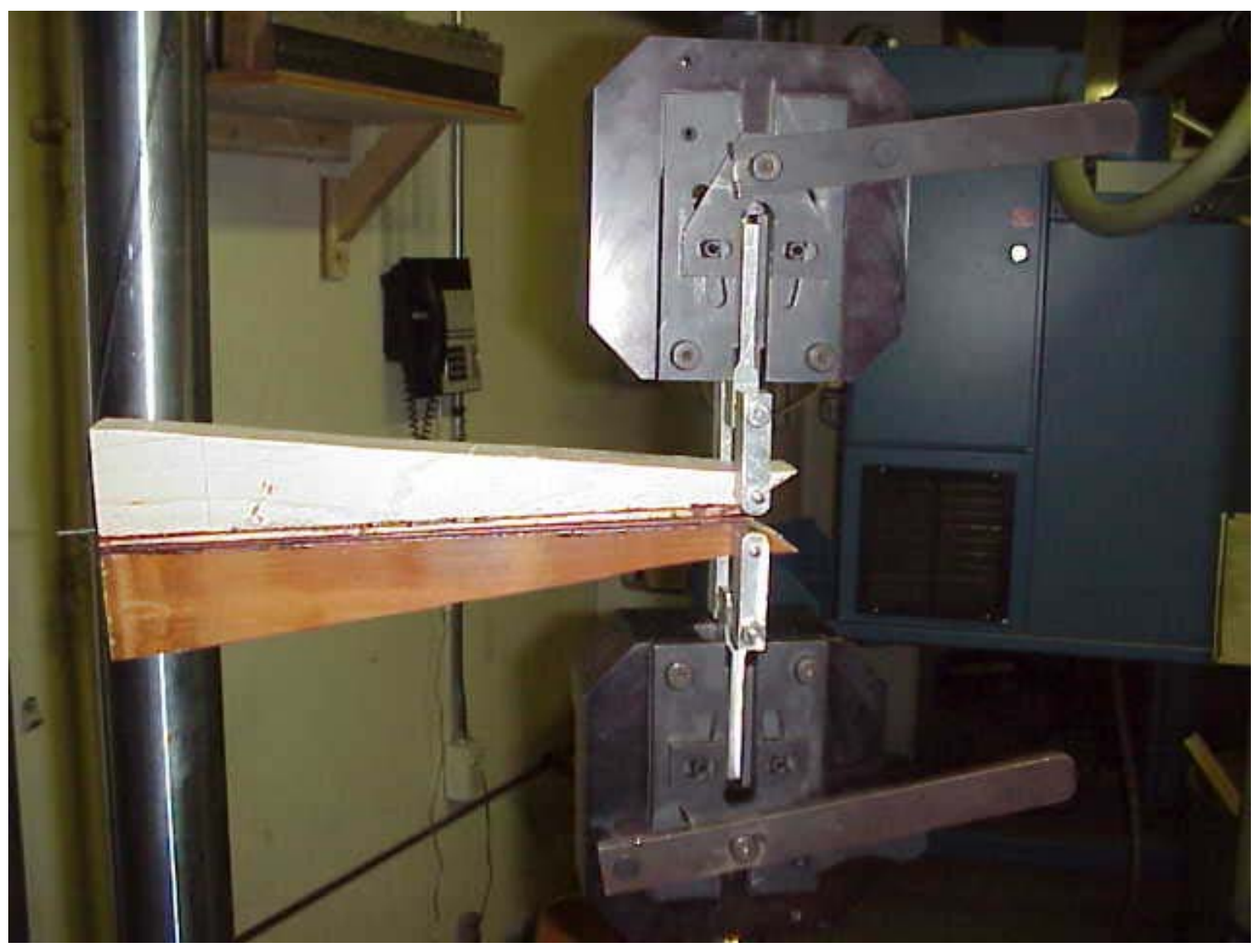

Figure 2.10 Photograph of a specimen under fatigue tests 


\section{CHAPTER 3}

\section{LOAD RATIO EFFECT}

\subsection{Introduction}

The load ratio $R$ is defined as the ratio of the minimum load $P_{\min }$ to maximum load $P_{\max }, R=P_{\min } / P_{\max }$. With this definition, $R=0$ is for zero-tension fatigue, $R=1$ is for a static load, and $R=-1$ is for fully reversed loading. The load ratio is also characterized in terms of the mean stress. A fatigue cycle of sinusoidal waveform with nonzero mean stress is shown in Fig. 3.1. The mean stress, the stress amplitude and the stress range for the case in Fig. 3.1 are defined respectively as

$$
\begin{aligned}
& \sigma_{m}=\frac{\sigma_{\min }+\sigma_{\max }}{2} \\
& \sigma_{a}=\frac{\sigma_{\max }-\sigma_{\min }}{2} \\
& \Delta \sigma=\sigma_{\max }-\sigma_{\min }
\end{aligned}
$$


where $\sigma_{\max }$ and $\sigma_{\min }$ are maximum and minimum stresses. The nomenclature for stress can also be applied in a similar manner to fracture toughness (either stress intensity factor $K$ or strain energy release rate $G$ ) and load $P$.

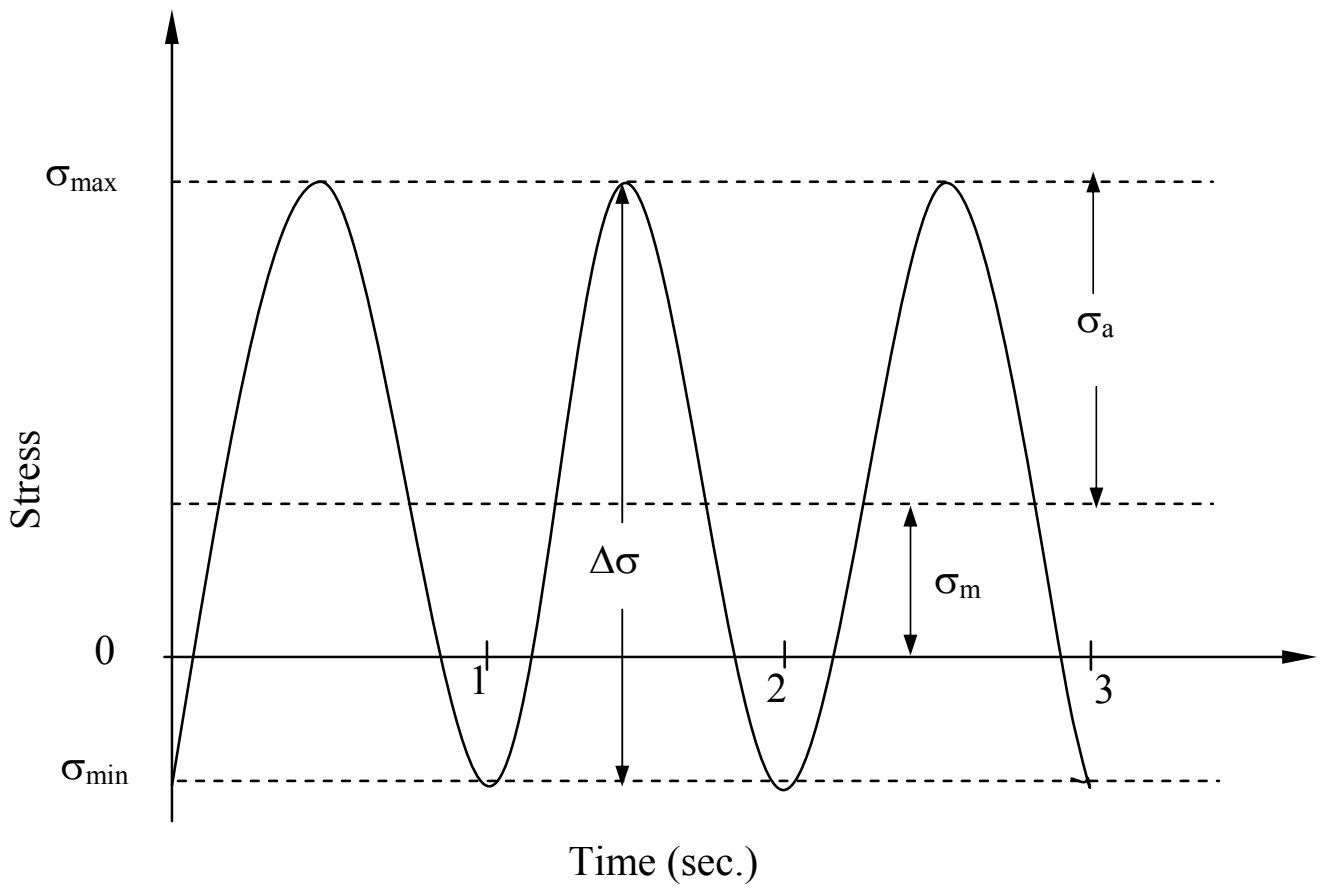

Figure 3.1 Nomenclature for stress parameters 
Load ratio $R$ is known to play an important role in influencing the fatigue behavior of engineering materials. It has been shown that the load ratio $R$ is a very significant parameter for the fatigue crack growth rate, particularly in Region II (see Fig. 1.1). For constant fracture toughness range, the crack growth rate could vary greatly with different load ratios. It is also well accepted that the threshold of the fracture toughness range is changed with different load ratios. Many researchers noticed that the original Power Law could not explain the load ratio effect, and therefore several modified Paris Law equations were proposed to include load ratio effect.

This chapter is concerned with the load ratio effect on fatigue fracture of red maple wood-phenolic FRP bonded interfaces using a contoured double cantilever beam (CDCB) specimen, and the pertinent literature review is summarized briefly next. Followed by the experimental program, data reduction techniques, proposed models to account for load ratio effect, and conclusions. 


\subsection{Literature Review}

The widely known empirical fatigue crack growth equation is the Paris Law or Power Law. It states that the crack growth rate $d a / d N$, where $a$ is a crack length and $N$ is the number of cycles, depends only on the amplitude of the stress intensity factor $\Delta K$. The Power Law equation is described as:

$$
\frac{d a}{d N}=C \Delta K^{m}
$$

where $C$ and $m$ are material constants that can be determined experimentally.

Sutton (1974) investigated fatigue behavior of an epoxy polymer with a tapered double cantilever beam (TDCB) specimen. Instead of amplitude of a stress intensity factor $\Delta K$, he used amplitude of an strain energy release rate, $\Delta G$, in his empirical equation as

$$
\frac{d a}{d N}=B(\Delta G)^{m}
$$

From linear elastic fracture mechanics concepts, the energy release rate is proportional to the square of the stress intensity factor, which leads to

$$
2 \Delta K K_{\text {mean }}=K_{\text {max }}^{2}-K_{\text {min }}^{2}=\frac{E}{\eta}(\Delta G)
$$


where $\eta=\left(1-v^{2}\right)$ for plane strain, $\eta=1$ for plane stress, $v=$ Poisson's ratio, and $K_{\text {mean }}=$ mean value of stress intensity factor. The range of the energy release rate $\Delta G$ is proportional to the product of $\Delta K$ and $K_{\text {mean }}$, and therefore, $\Delta G$ itself includes the mean stress intensity factor effect (or load ratio effect). Some researchers have noted that the $\log -\log$ plots of $d a / d N$ versus $\Delta K$ resulted in different relationships for different load ratios. However, when a graph was produced for crack growth rate $d a / d N$ versus the range of energy release rate $\Delta G$, one single straight line was able to fit the data for all different load ratios. For example, Sutton (1974) showed that the range of energy release rate is a better parameter to evaluate the fatigue resistance of epoxy polymer or other similar resins, which could be used for interface adhesives or matrix binders for composite materials. Moreover, the TDCB specimen, for which the strain energy release rate is independent of crack length, was proved suitable for fatigue test studies.

Ishiyama et al. (1987) studied the effect of stress ratio on crack extension rate of a fine-grained isotropic nuclear graphite. The tests were performed at a loading rate of 251 $\mathrm{N} / \mathrm{s}$ with a tapered double cantilever beam (TDCB) specimen. Five load ratios of 0.0, 0.3, $0.5,0.65$ and 0.80 were employed for the fatigue tests. The crack extension was measured with a comparator to detect changes in crack length of more than $10 \mu \mathrm{m}$. For a given load ratio $R$, crack extension rate $d a / d N$ was expressed in the form of the original Paris Law equation, and it was found that the crack growth rate $d a / d N$ depended strongly on $R$. The threshold stress intensity factor range $\Delta K_{t h}$ was used to explain the load ratio effect, by a formula expressed as 


$$
\frac{d a}{d N}=C\left(\Delta K-\Delta K_{t h}\right)^{n}
$$

With the exponent $n=5.7$, they reported that the experimental data were represented well by a single straight line for different load ratios. However, there were some differences between the data for $R=0.0$ and 0.8 , which may indicate that fracture mode changes with increasing $R$. The authors believed that the crack growth rate $d a / d N$ is dependent on $\Delta K$ for low values of $R$ and almost entirely on $K_{\max }$ for high values of $R$.

Hojo et al. (1987) studied the stress or load ratio effect on unidirectional laminates 914C prepregs (T300/914) and Toray P305 prepregs (T300/\#2500). The conventional double cantilever beam (DCB) specimen was used, and the compliance of the specimen was expressed as

$$
C=D a^{n}
$$

The coefficient $n=2.21$ was obtained by a least squares approximation. A power function of $\Delta K$ was plotted in the region where $d a / d N$ was larger than about $5 \times 10^{-10}$ $\mathrm{m} /$ cycle, and the exponent of the power function was greater than 14 , which is much higher than that of metallic materials. The crack growth rate was plotted versus the stress intensity factor range, $\Delta K$, the maximum energy release rate, $G_{\max }$, and the range of energy release rate, $\Delta G$. It was reported that the influence of stress, or load, ratio became smallest for the case of energy release rate range, and the effect of stress ratio was almost 
negligible for the $914 \mathrm{C}$ laminates when using the energy release rate range $\Delta G$ as the controlling parameter. Although for the $\mathrm{P} 305$ laminates the scatter of $d a / d N$ versus $\Delta G$ was much smaller within the chosen Power Law region than that for $d a / d N$ versus $\Delta K$ or $G_{\max }$, the load ratio effect still remained non-negligible and another parameter was needed to explain load ratio effect by defining the equivalent stress intensity range $\Delta K_{\text {eq }}$, as

$$
\Delta K_{e q}=\Delta K(1-R)^{-\gamma}
$$

or

$$
\Delta K_{e q}=\Delta K^{(1-\gamma)} K_{\max }^{\gamma}
$$

and the material constant $\gamma$ is obtained by fitting experimental data with

$$
\Delta K=\Delta K_{0}(1-R)^{\gamma}
$$

where $\Delta K$ and $\Delta K_{0}$ are the threshold values of stress intensity factor range at load ratios $R$ and $R=0$, respectively. The authors reported that $\gamma$ equal to 0.51 by fitting experimental data. A phenomenological fatigue growth law was proposed as

$$
d a / d N=A \Delta K_{e q}^{m}
$$


For $m=26$, the scatter of data points for the P305 laminates merged into a band of relatively straight line using this model.

Atodaria et al. (1997 and 1999) studied the load ratio effect of a randomly oriented short carbon fiber reinforced polyether ether ketone (PEEK) composite and a glass fabric reinforced epoxy laminate under tension-tension cyclic loading. Fatigue tests were conducted at a constant amplitude sinusoidal waveform and a frequency of $2 \mathrm{~Hz}$ for different load ratios. Compact tension (CT) and double cantilever beam (DCB) specimens were used. They assumed that every load level from minimum to maximum load has a certain effect on fatigue crack propagation and the damage development and accumulation occurs in the entire fatigue cycle. Lower load values may have minor contribution to progressive crack growth; higher load values would have more contribution to crack growth. Based on this assumption, they proposed a modified Power Law equation, which takes into account the progressive crack propagation process as:

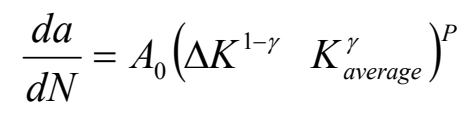

Where,

$$
\begin{aligned}
& K_{\text {average }}=\left[(1 / n) \sum_{K_{t h}}^{K_{\max }} K^{w}\right]^{1 / w} \\
& n=\text { number of divided regions from } K_{t h} \text { to } K_{\max } \\
& w=\text { weight factor } \\
& A_{0}, \gamma, P=\text { material constants }
\end{aligned}
$$


$K_{t h}=$ the threshold stress intensity factor below which no crack propagation occurs

It was determined that $A_{0}=5.4 \times 10^{-20}, \gamma=0.5$ and $P=17.4$ for the randomly oriented short carbon fiber reinforced PEEK composite. They found that the strain energy release rate is more suitable than the stress intensity factor for characterizing the Mode-I fracture of laminated composites under cyclic loading conditions. The Paris Law equation is expressed in terms of the strain energy release rate as

$$
\frac{d a}{d N}=B\left\{(\sqrt{G})_{\text {average }}^{\gamma} \cdot(\Delta \sqrt{G})^{1-\gamma}\right\}^{P}
$$

where,

$$
\begin{aligned}
& \Delta \sqrt{G}=\sqrt{G_{\max }}-\sqrt{G_{\min }} \\
& (\sqrt{G})_{\text {average }}=\left[\frac{1}{n} \sum_{(\sqrt{G})_{\text {lih }}}^{(\sqrt{G})_{\max }}(\sqrt{G})^{w}\right]^{1 / w}
\end{aligned}
$$

They obtained $A_{0}=2.32 \times 10^{-21}, \gamma=0.6$ and $P=14.612$ for the glass fabric reinforced epoxy laminate. Comparing with metals, composites have higher exponent values. The proposed model proved to be capable of unifying the fatigue crack growth rate at different load ratios into a single Power Law equation. 
Arad, Radon, and Culver (1971) studied the effect of the mean stress intensity factor on fatigue crack propagation in polymethylmethacrylate (PMMA). Repeated tension tests were carried out on sheet specimens containing a central through-thickness crack. Tests were conducted at room temperature with a frequency of $5 \mathrm{~Hz}$ and a sine waveform. The results have shown that both $\Delta K$ and $K_{m}$ were of considerable importance in influencing fatigue crack growth rates in PMMA and the mean stress intensity factor cannot be discarded as having only secondary influence on crack growth. Based on the experimental data, a relationship of the following form has been proposed

$$
\frac{d(2 a)}{d N}=\beta\left(K_{\max }^{2}-K_{\min }^{2}\right)^{n}
$$

The expression $K_{\max }^{2}-K_{\min }^{2}=2 \Delta K K_{m}$ includes the influence of the mean level of the stress intensity factor. It was found that constants $n=2.5$ and $\log \beta=10^{-12.37}$ fitted the data for PMMA. It was shown that the crack growth rate is constant if $K_{\max }^{2}-K_{\min }^{2}$ is constant over the range of values tested. Arad et al. (1972) applied this model to the analysis of fatigue failure in Nylon 6.6, and good agreement between Eq. (3.15) and their experimental data was reported by the authors. Radon et al. (1974) applied Eq. (3.15) to metal fatigue process. Fatigue tests with a frequency between 0.1 and $1 \mathrm{~Hz}$ were carried out on two aluminum alloys, B $26 \mathrm{~S}$ and RR 58. They found that the crack growth rate could be determined from a single set of data irrespective of the values of stress intensity factor range $\Delta K$ and load ratio $R$. A more generalized form of Eq. (3.15) was given by 
using strain energy release rate $G$, the contour integral $J$ and the crack opening displacement (COD):

$$
\frac{d(2 a)}{d N}=M A^{n}
$$

where $A=G_{\max }-G_{\min }=J_{\max }-J_{\text {min }}=C O D_{\max }-C O D_{\min }, M$ and $A$ are material constants.

Branco, Radon and Culver (1975b) studied an aluminum alloy RR58 for fatigue crack growth rate phenomena. Cyclic tension tests were conducted at $0.15 \mathrm{~Hz}$ and room temperature with a contoured double cantilever beam (CDCB) specimen. In order to separate the individual effects of the mean value of stress intensity factor $K_{m}$ and stress intensity factor range $\Delta K$, they conducted two series of tests. First, $\Delta K$ was constant, $K_{m}$ was varied; second, $K_{m}$ was constant; $\Delta K$ was varied. These two series of tests indicated that $K_{m}$ and $\Delta K$ exerted distinct influences on crack growth rates of the alloy RR58. Considering the influence of fatigue threshold $\Delta K_{t h}$ on crack growth rate in Region I, plus the critical parameter of stress intensity factor $K_{I C}$ at the instability point of Region III (see Fig. 1.1), and the facts that

$$
\left(2 \Delta K K_{m}-2 \Delta K_{t h} K_{m}\right) \rightarrow 0
$$

when $d a / d N$ approaches zero, and 


$$
\left(K_{1 C}-K_{\max }\right) \rightarrow 0
$$

when $d a / d N$ approaches infinity, Branco et al. (1975) proposed a Paris Law equation as

$$
\frac{d a}{d N}=A\left[\frac{2 K_{m}\left(\Delta K-\Delta K_{t h}\right)}{K_{1 C}^{2}-K_{\max }^{2}}\right]^{\alpha}
$$

with the constants $A=3.16 \times 10^{-5}$ in/cycle and $\alpha=1.36$ determined from the experimental data. Eq. (3.19) was reported to represent the crack process very well.

The modified Paris Law equations presented above as proposed by others are attempting to explain the load ratio effect, where most of them are obtained from and/or applied to conventional double cantilever beam (DCB) and contoured or tapered double cantilever beam (C/TDCB) specimens. For a comparative study and the readers' convenience, the expressions are sequentially shown in Table 3.1 with the description of specimen type, materials used, authors and year. 
Table 3.1 Proposed modified Paris Law equations including load ratio effect

\begin{tabular}{|c|c|c|c|}
\hline Modified Paris Law & $\begin{array}{l}\text { Specimen } \\
\text { Type }\end{array}$ & Materials & $\begin{array}{l}\text { Authors and } \\
\text { Year }\end{array}$ \\
\hline$\frac{d a}{d N}=B(\Delta G)^{m}$ & TDCB & An epoxy resin polymer & Sutton, 1974 \\
\hline$\frac{d a}{d N}=C\left(\Delta K-\Delta K_{t h}\right)^{n}$ & TDCB & $\begin{array}{l}\text { A fine-grained isotropic } \\
\text { nuclear graphite IG-11 }\end{array}$ & $\begin{array}{l}\text { Ishiyama et } \\
\text { al., } 1987\end{array}$ \\
\hline $\begin{array}{c}\frac{d a}{d N}=A \Delta K_{e q}^{m} \\
\Delta K_{e q}=\Delta K(1-R)^{-\gamma} \\
\Delta K_{e q}=\Delta K^{(1-\gamma)} K_{\max }^{\gamma}\end{array}$ & DCB & $\begin{array}{c}\text { A unidirectional laminates } \\
\text { 914C prepregs (T300/914) } \\
\text { and Toray P305 prepregs } \\
\text { (T300/\#2500) }\end{array}$ & $\begin{array}{c}\text { Hojo et al., } \\
1987\end{array}$ \\
\hline$\frac{d a}{d N}=A\left(\sqrt{\Delta K \cdot K_{\text {average }}}\right)^{P}$ & $\begin{array}{l}\text { Compact } \\
\text { tension } \\
\text { (CT) }\end{array}$ & $\begin{array}{l}\text { A randomly oriented short } \\
\text { carbon fiber reinforced } \\
\text { PEEK composite }\end{array}$ & $\begin{array}{c}\text { Atodaria et al., } \\
1997\end{array}$ \\
\hline$\frac{d a}{d N}=B\left((\sqrt{G})_{\text {average }}^{\gamma} \cdot(\Delta \sqrt{G})^{1-\gamma}\right\}^{P}$ & $\mathrm{DCB}$ & $\begin{array}{l}\text { A glass fabric reinforced } \\
\text { laminated composite }\end{array}$ & $\begin{array}{c}\text { Atodaria et al., } \\
1999\end{array}$ \\
\hline$\frac{d a}{d N}=A\left[\frac{2 K_{m}\left(\Delta K-\Delta K_{t h}\right)}{K_{1 C}^{2}-K_{\max }^{2}}\right]^{m}$ & $\mathrm{CDCB}$ & An Al-alloy RR58 & $\begin{array}{c}\text { Branco et al., } \\
1975\end{array}$ \\
\hline$\frac{d a}{d N}=A\left[K_{\max }^{2}-K_{\min }^{2}\right]^{m}$ & $\begin{array}{l}\text { Central } \\
\text { notched } \\
\text { plate }\end{array}$ & $\begin{array}{l}\text { A polymethylmethacrylate } \\
\text { (PMMA) }\end{array}$ & $\begin{array}{c}\text { Arad et al., } \\
1971\end{array}$ \\
\hline
\end{tabular}




\subsection{Fatigue Test}

This section describes materials and specimen used and test procedures.

\subsubsection{Material and specimen}

A contoured double cantilever beam (CDCB) specimen designed by the RayleighRitz method was used for fatigue tests of red maple wood-phenolic FRP composite bonded interfaces. The CDCB specimen is contoured to achieve a constant rate of compliance change with respect to crack length, $d C / d a$. The details of the specimen design and applications for wood-FRP interface fracture studies are given in several papers by Davalos et al. (1997, 1998a, 1998b, 2000a, 2000b) and Qiao et al. (2000). The geometric details of the CDCB specimen in this study are shown in Fig. 2.4, and the adherends consist of red maple wood and pultruded phenolic FRP composite with the contoured portions made of laminated veneer lumber (LVL). The lay-up of the pultruded phenolic FRP laminate is shown in Figure 2.3, and the mechanical properties of red maple and phenolic FRP are summarized in Table 2.2. The adhesive used for bonding red maple wood-phenolic FRP interface was Resorcinol-Formaldehyde from INDSPEC Chemical Corp., Pittsburgh, PA.

The average of the $d C / d a$ value obtained by the Rayleigh-Ritz method and finite element modeling was $27.17 \times 10^{-5} \mathrm{lb}^{-1}$. Compliance calibration tests were conducted for several wood-FRP CDCB samples, and the average $d C / d a$ value obtained from 
experiments was $29.59 \times 10^{-5} \mathrm{lb}^{-1}$. The experimental result of the $d C / d a$ value is used to calculate strain energy release rate $G$ in the present study.

\subsubsection{Test procedure}

Cyclic tension-tension fatigue tests were conducted in a computer controlled MTS servo hydraulic machine. The load ratio $R$ (the ratio of minimum load to the maximum load) was kept constant during the testing of each group of samples. Three groups of tests for load ratios $R$ of $0.1,0.3$ and 0.5 were completed under load control mode (see Fig. 3.2). The applied loads for each group of tests are listed in Tables 3.2, 3.3 and 3.4. A frequency of $1 \mathrm{~Hz}$ and a sinusoidal waveform (see Fig. 2.7) were used for all the tests. The tests in the MTS machine were controlled by pre-programmed software, which was also used as a data acquisition system. Since only the relative value of crack opening displacement (COD) is needed, a crosshead displacement instead of an extensometer displacement was used to measure the COD. The test environment was room temperature and open laboratory conditions for all the samples. 


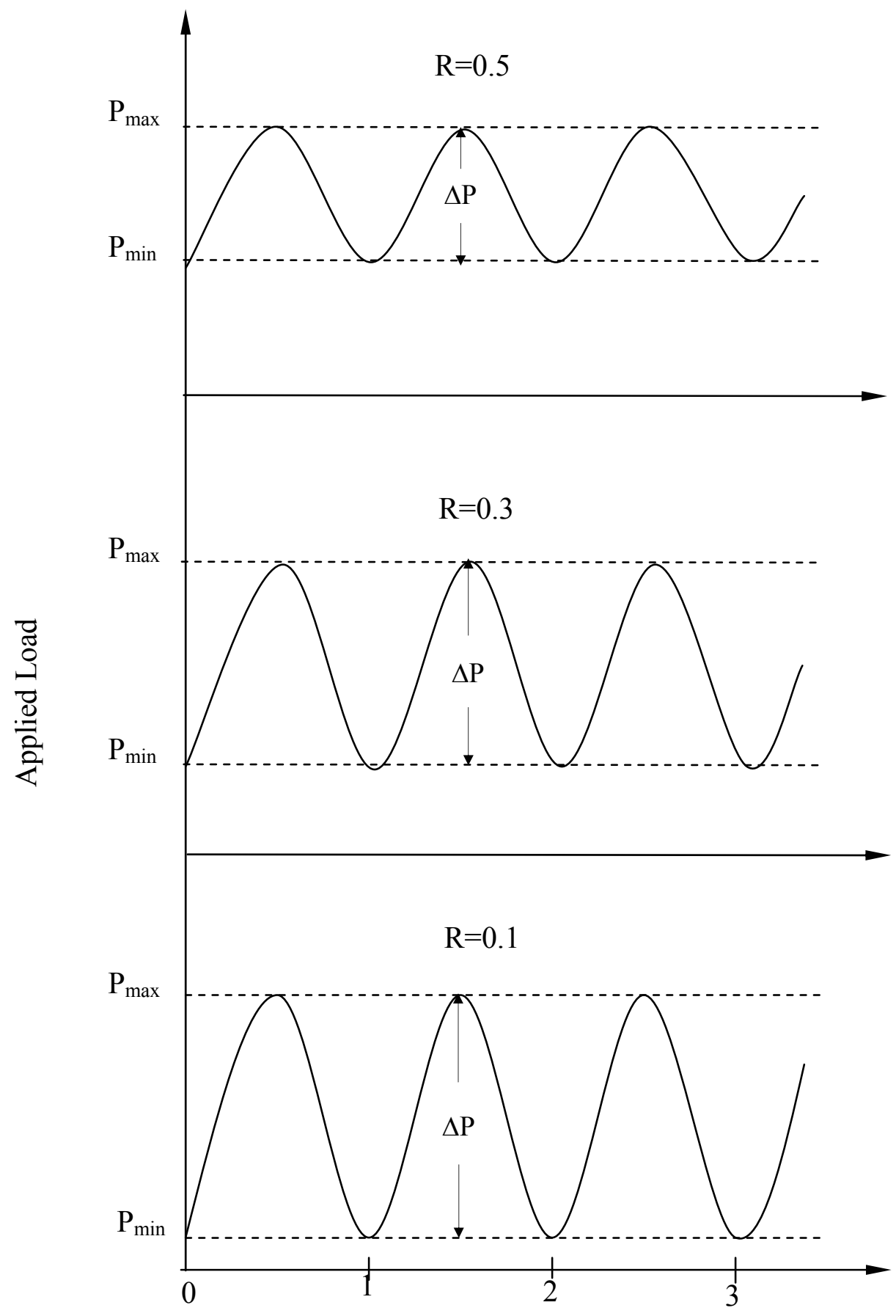

Time (sec.)

Fig. 3.2 A schematic illustration of cyclic loading at different load ratios 
Table 3.2 Applied load for load ratio $\mathrm{R}=0.1$

\begin{tabular}{|c|c|c|c|c|}
\hline $\boldsymbol{P}_{\min }$ & $\boldsymbol{P}_{\max }$ & $\Delta \boldsymbol{P}=\boldsymbol{P}_{\max }-\boldsymbol{P}_{\min }$ & $\boldsymbol{R}$ & $\boldsymbol{P}_{\text {mean }}$ \\
\hline 13 & 130 & 117 & 0.1 & 71.5 \\
\hline 12 & 120 & 108 & 0.1 & 66 \\
\hline 11 & 110 & 99 & 0.1 & 60.5 \\
\hline 10 & 100 & 90 & 0.1 & 55 \\
\hline 9 & 90 & 81 & 0.1 & 49.5 \\
\hline 8.5 & 85 & 76.5 & 0.1 & 46.75 \\
\hline 8 & 80 & 72 & 0.1 & 44 \\
\hline
\end{tabular}

Table 3.3 Applied load for load ratio $\mathrm{R}=0.3$

\begin{tabular}{|c|c|c|c|c|}
\hline $\boldsymbol{P}_{\min }$ & $\boldsymbol{P}_{\max }$ & $\Delta \boldsymbol{P}=\boldsymbol{P}_{\max }-\boldsymbol{P}_{\min }$ & $\boldsymbol{R}$ & $\boldsymbol{P}_{\text {mean }}$ \\
\hline 39 & 130 & 91 & 0.3 & 84.5 \\
\hline 37.5 & 125 & 87.5 & 0.3 & 81.25 \\
\hline 36 & 120 & 84 & 0.3 & 78 \\
\hline 33 & 110 & 77 & 0.3 & 71.5 \\
\hline 30 & 100 & 70 & 0.3 & 65 \\
\hline 27 & 90 & 63 & 0.3 & 58.5 \\
\hline 25.5 & 85 & 59.5 & 0.3 & 55.25 \\
\hline 24 & 80 & 56 & 0.3 & 52 \\
\hline
\end{tabular}


Table 3.4 Applied load for load ratio $\mathrm{R}=0.5$

\begin{tabular}{|c|c|c|c|c|}
\hline $\boldsymbol{P}_{\min }$ & $\boldsymbol{P}_{\max }$ & $\Delta \boldsymbol{P}=\boldsymbol{P}_{\max }-\boldsymbol{P}_{\min }$ & $\boldsymbol{R}$ & $\boldsymbol{P}_{\text {mean }}$ \\
\hline 65 & 130 & 65 & 0.5 & 97.5 \\
\hline 60 & 120 & 60 & 0.5 & 90 \\
\hline 55 & 110 & 55 & 0.5 & 82.5 \\
\hline 50 & 100 & 32.5 & 0.5 & 75 \\
\hline 47.5 & 95 & 47.5 & 0.5 & 71.25 \\
\hline 45 & 90 & 45 & 0.5 & 67.5 \\
\hline 42.5 & 85 & 42.5 & 0.5 & 63.75 \\
\hline 40 & 80 & 40 & 0.5 & 60 \\
\hline
\end{tabular}

\subsection{Results}

This section includes discussions of data collection and processing, measurement of crack propagation by the compliance method, several modified Paris Law models to fit the data, exploration of crack closure effect, and description of failure surfaces.

\subsubsection{Experimental data processing}

The experimental results of the crack opening displacement (COD) versus the number of cycles for phenolic FRP-red maple wood bonded interface samples are summarized and discussed in this section. The load displacement data were continuously recorded and saved into a *.txt file. The data collection rate was 10 data points per second. The displacement data point corresponding to the maximum load per cycle is needed and extracted. The data file was too large to analyze for lower load levels since the Microsoft Excel spreadsheet can only deal with the maximum of 65536 rows of data. 
One way of solving this problem was to split the data file to smaller text files (about 800 KB each) so that we could use Microsoft Excel spreadsheet to analyze it. A typical diagram of COD versus number of cycles for a CDCB specimen is given in Fig 3.3, which is obtained under $65 \mathrm{lb}$. minimum load and $130 \mathrm{lb}$. maximum load with a $1 \mathrm{~Hz}$ frequency and sinusoidal waveform.

As noticed by many researchers, fatigue ultimate failure occurs suddenly. The phenolic FRP-red maple wood bonded interface under constant cyclic loading demonstrated a catastrophic failure nature (see Fig. 3.3). In the beginning, twenty-four specimens were fabricated and then tested to obtain the COD propagation rate for different load ratios. Several problems were encountered during the testing; some specimens were broken during the set up process to achieve the desired load range, because the MTS machine had a tendency to jump and pull suddenly on the loading grips. Therefore, a try-on specimen was needed before the actual test and another twenty-four extra specimens were prepared. The loading details are given in Tables 3.2, 3.3 and 3.4. The experimental data of COD versus number of cycles of these tests were analyzed. Some of the bad results were discarded and tests were preformed again. The processed data of COD versus number of cycles are shown in Figs. 3.4 through 3.10 for load ratio of 0.1, Figs. 3.11 through 3.18 for load ratio of 0.3, and Figs. 3.19 through 3.26 for load ratio of 0.5 in an ascending loading order. Since only the intermediate region of fatigue process is of concerned, Figs. 3.4 through 3.26 show the processed data of stable COD propagation range versus number of cycles. The part of data at the beginning for opening the pre-crack region and at the end of catastrophic failure of the specimen is omitted. The 
COD propagation rates $d C O D / d N$ are obtained from adding the linear "trendline" of Microsoft Excel for these specimens. They are listed in Tables 3.5, 3.6 and 3.7 corresponding to load ratios of $0.1,0.3$ and 0.5 for transformation of the crack propagation rate $d a / d N$.

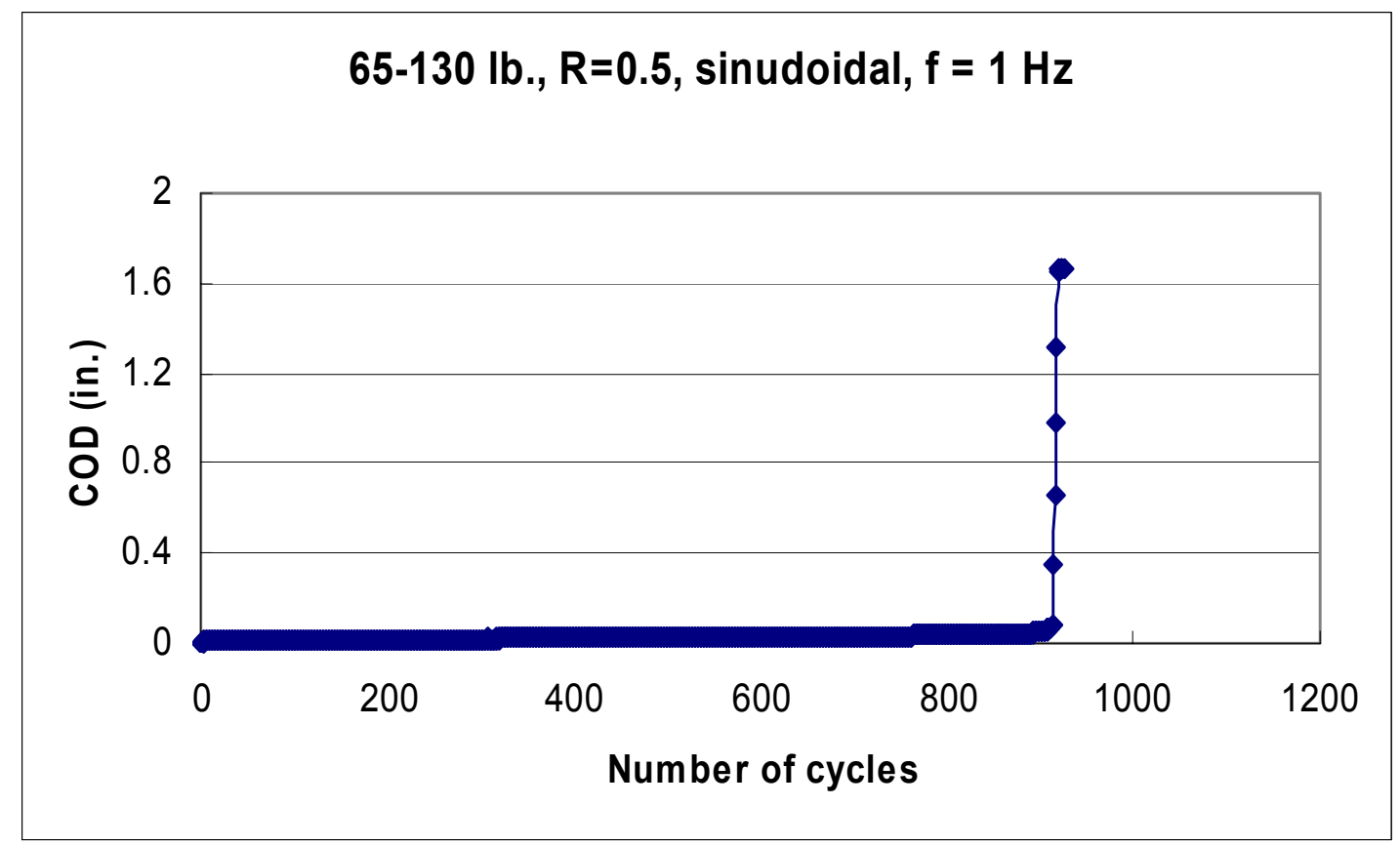

Fig. 3.3 A typical diagram of COD versus number of cycles for a CDCB specimen 


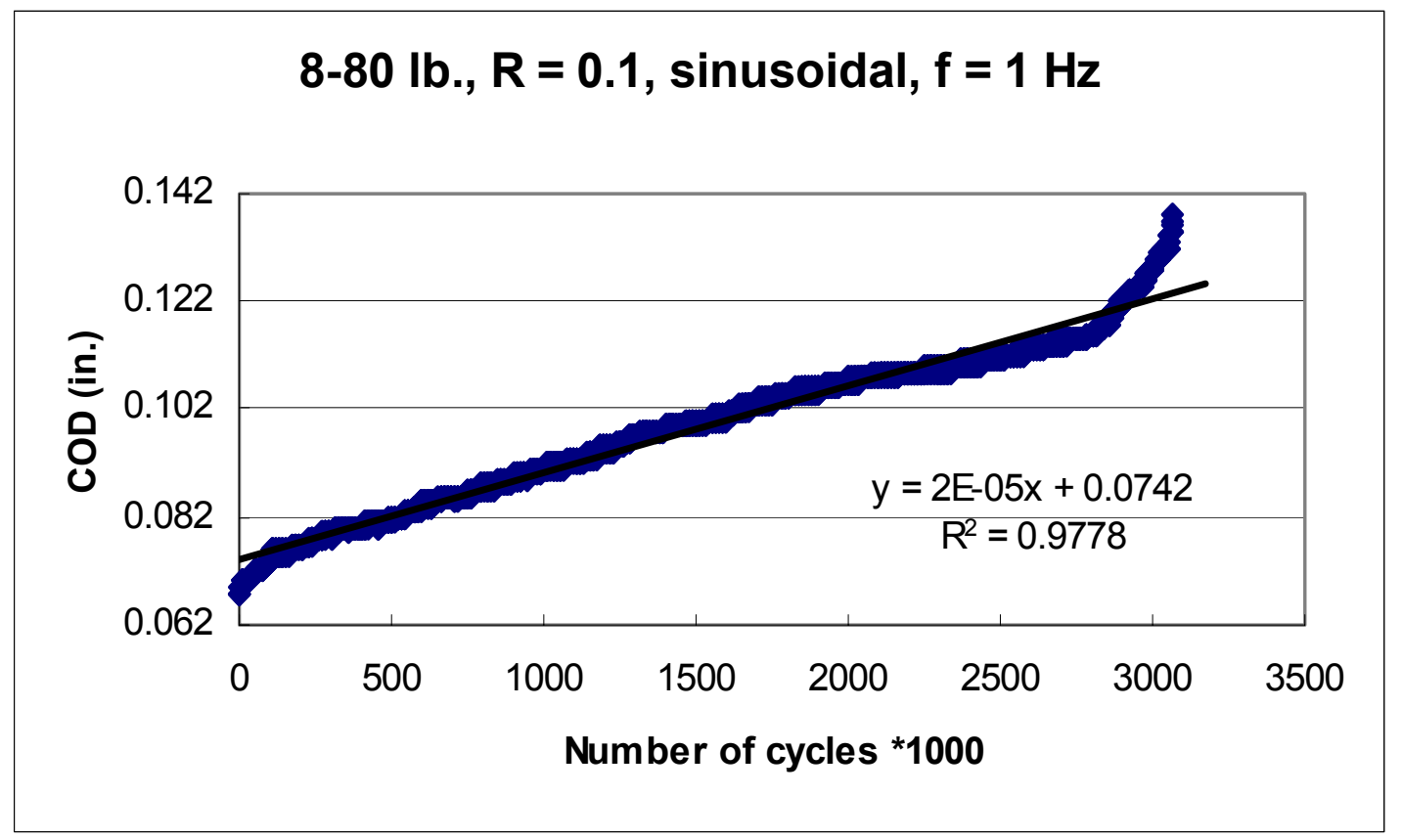

Fig. 3.4 COD versus number of cycles for a CDCB specimen under 8-80 lb. cyclic loading with a frequency of $1 \mathrm{~Hz}$, load ratio $R=0.1$ and sinusoidal waveform 


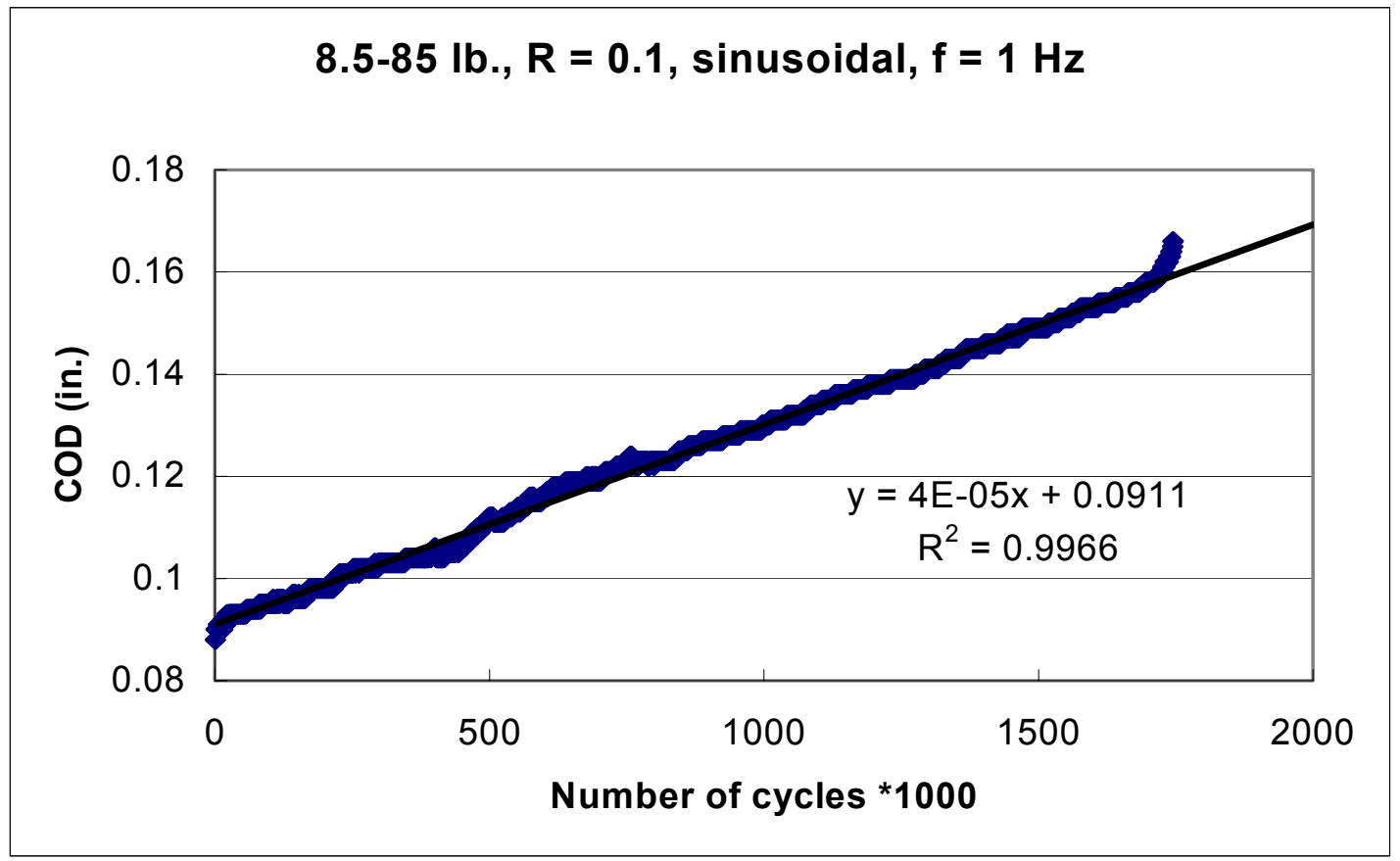

Fig. 3.5 COD versus number of cycles for a CDCB specimen under 8.5-85 lb. cyclic loading with a frequency of $1 \mathrm{~Hz}$, load ratio $R=0.1$ and sinusoidal waveform 


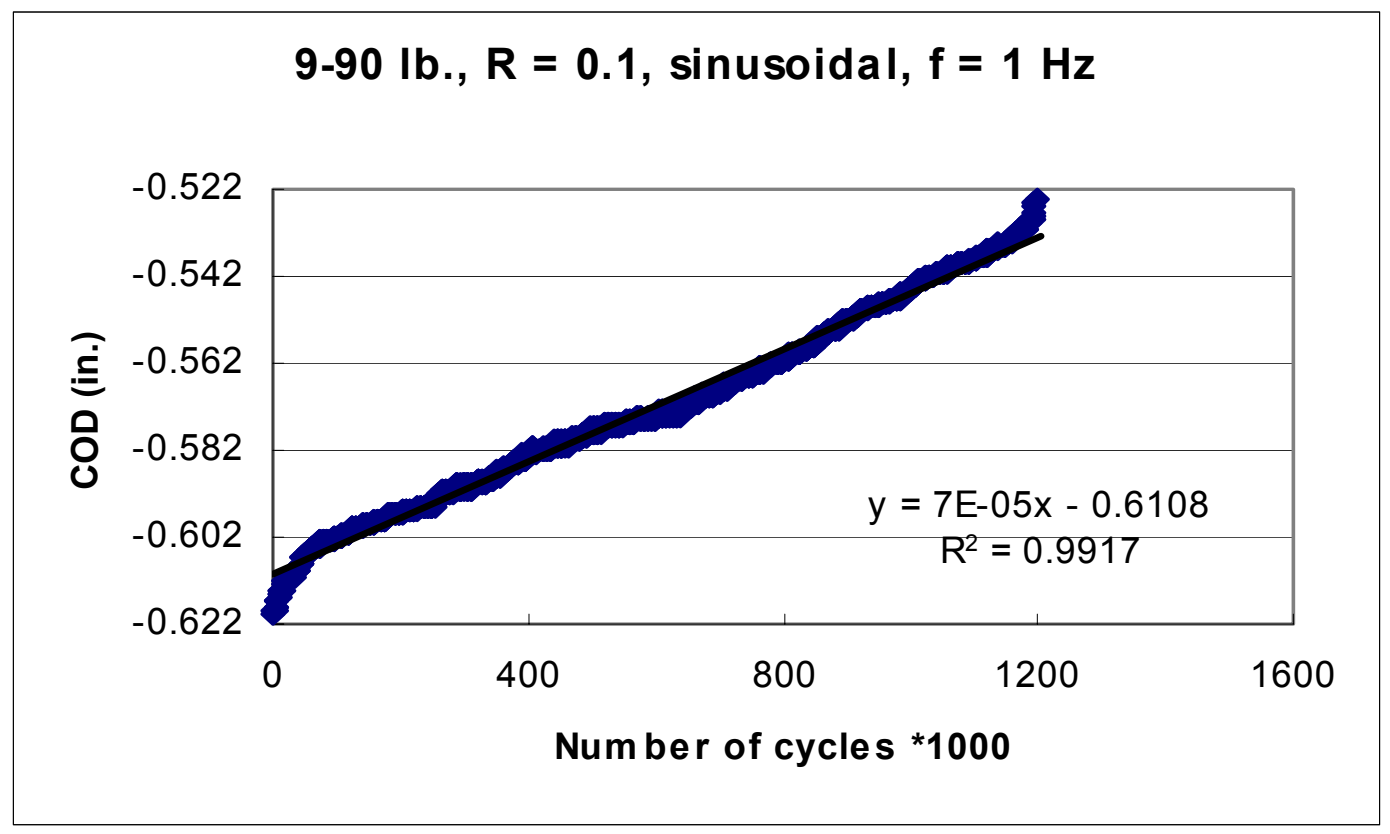

Fig. 3.6 COD versus number of cycles for a CDCB specimen under 9-90 lb. cyclic loading with a frequency of $1 \mathrm{~Hz}$, load ratio $R=0.1$ and sinusoidal waveform 


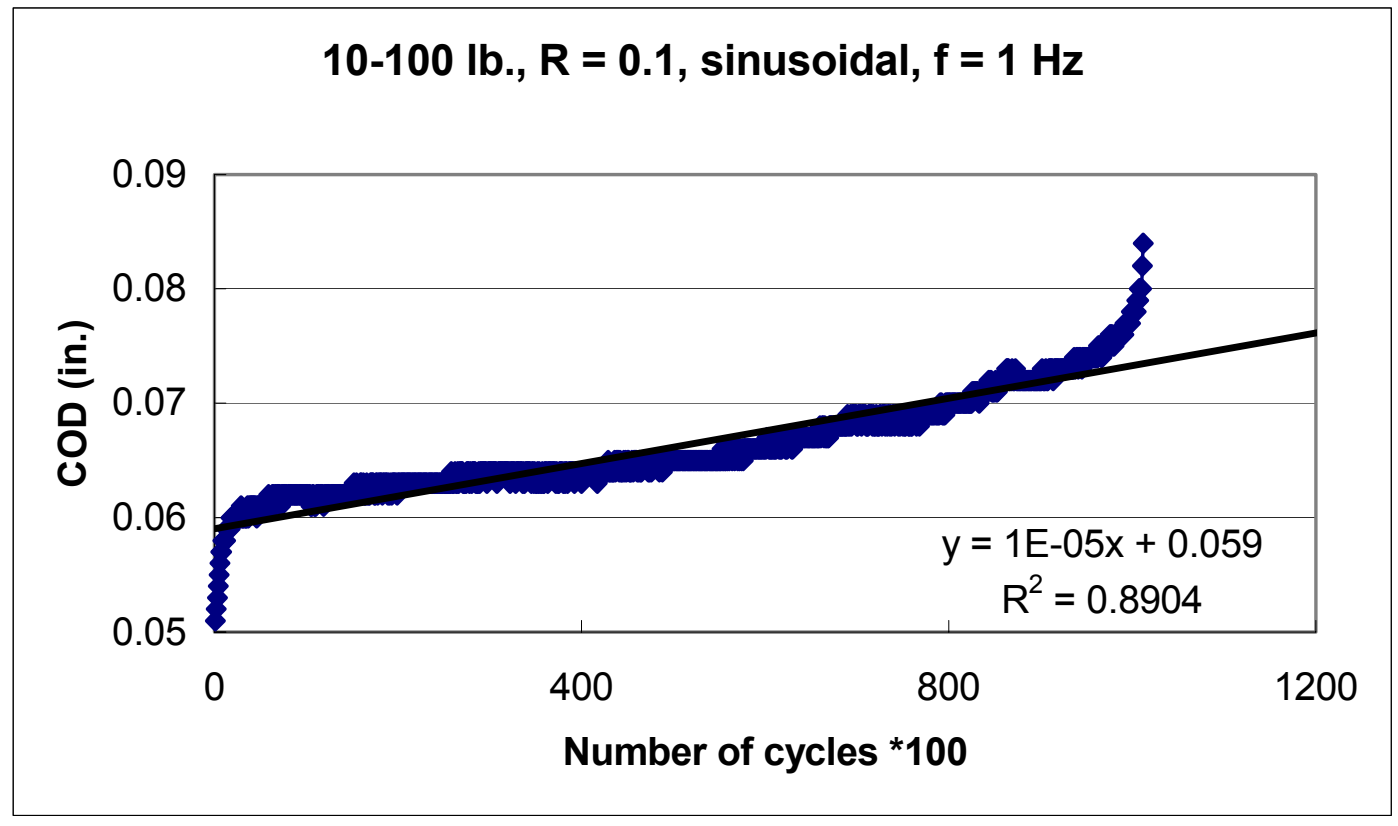

Fig. 3.7 COD versus number of cycles for a CDCB specimen under 10-100 lb. cyclic loading with a frequency of $1 \mathrm{~Hz}$, load ratio $R=0.1$ and sinusoidal waveform 


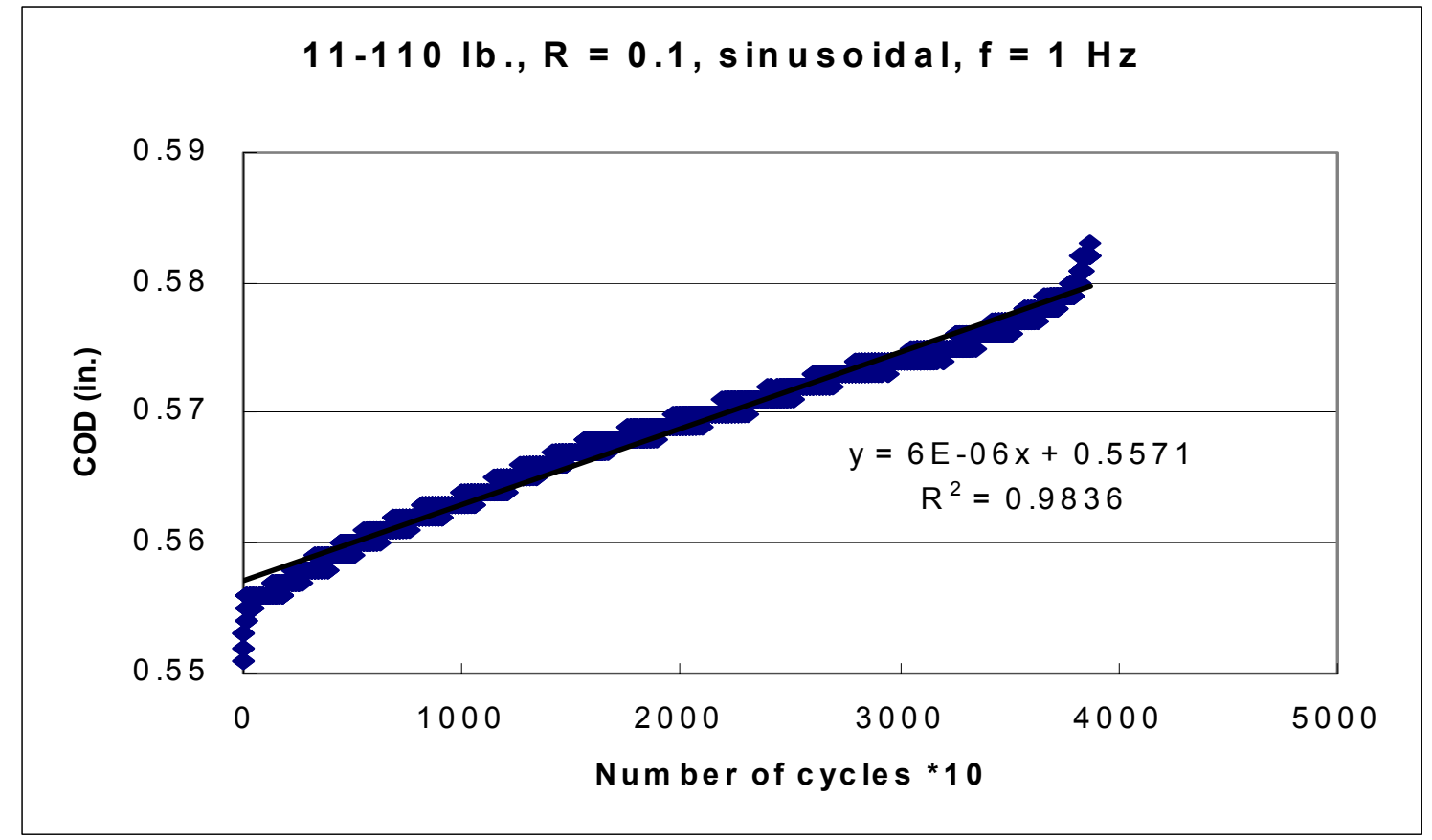

Fig. 3.8 COD versus number of cycles for a CDCB specimen under 11-110 lb. cyclic loading with a frequency of $1 \mathrm{~Hz}$, load ratio $R=0.1$ and sinusoidal waveform 


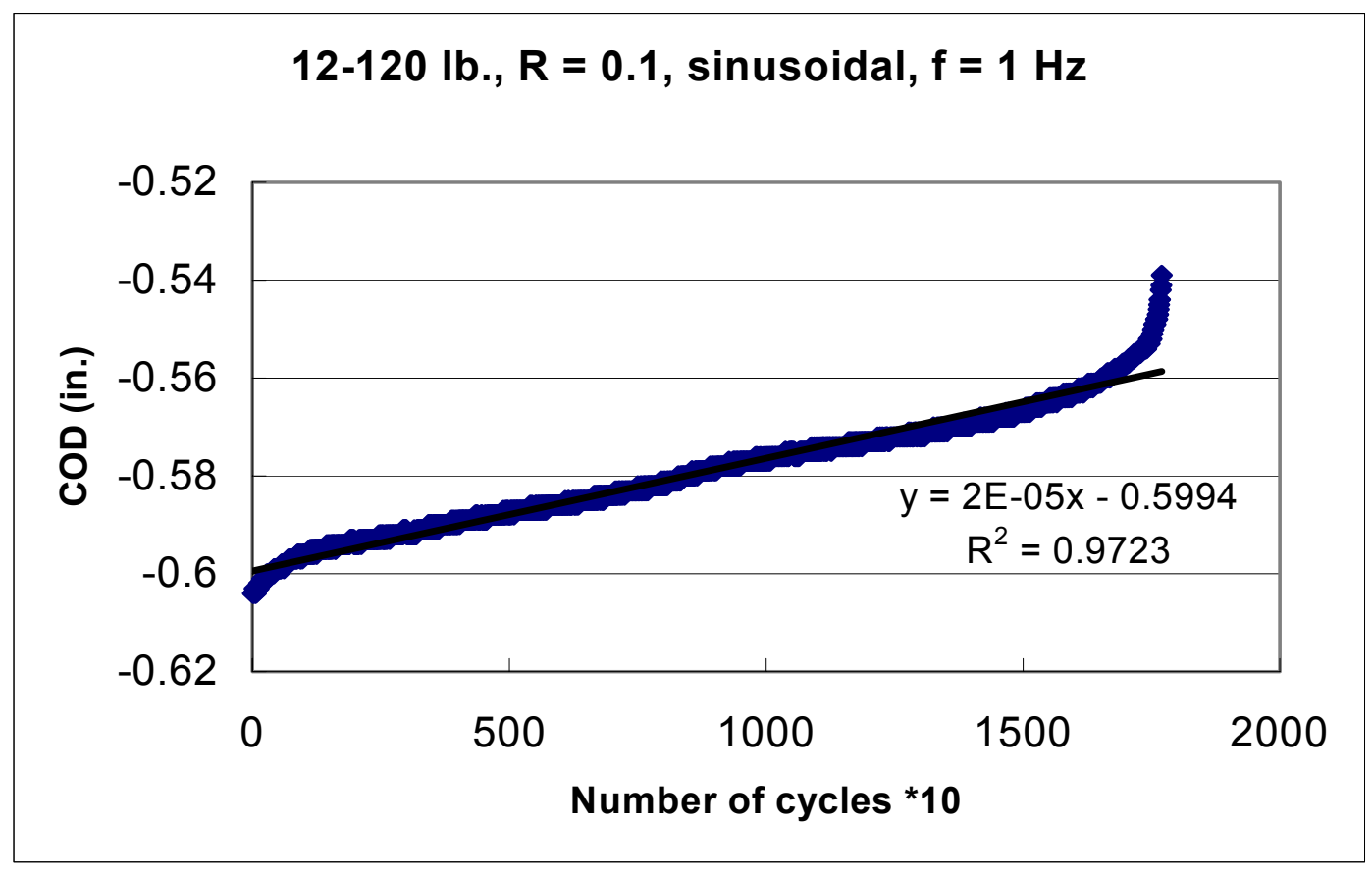

Fig. 3.9 COD versus number of cycles for a CDCB specimen under 12-120 lb. cyclic loading with a frequency of $1 \mathrm{~Hz}$, load ratio $R=0.1$ and sinusoidal waveform 


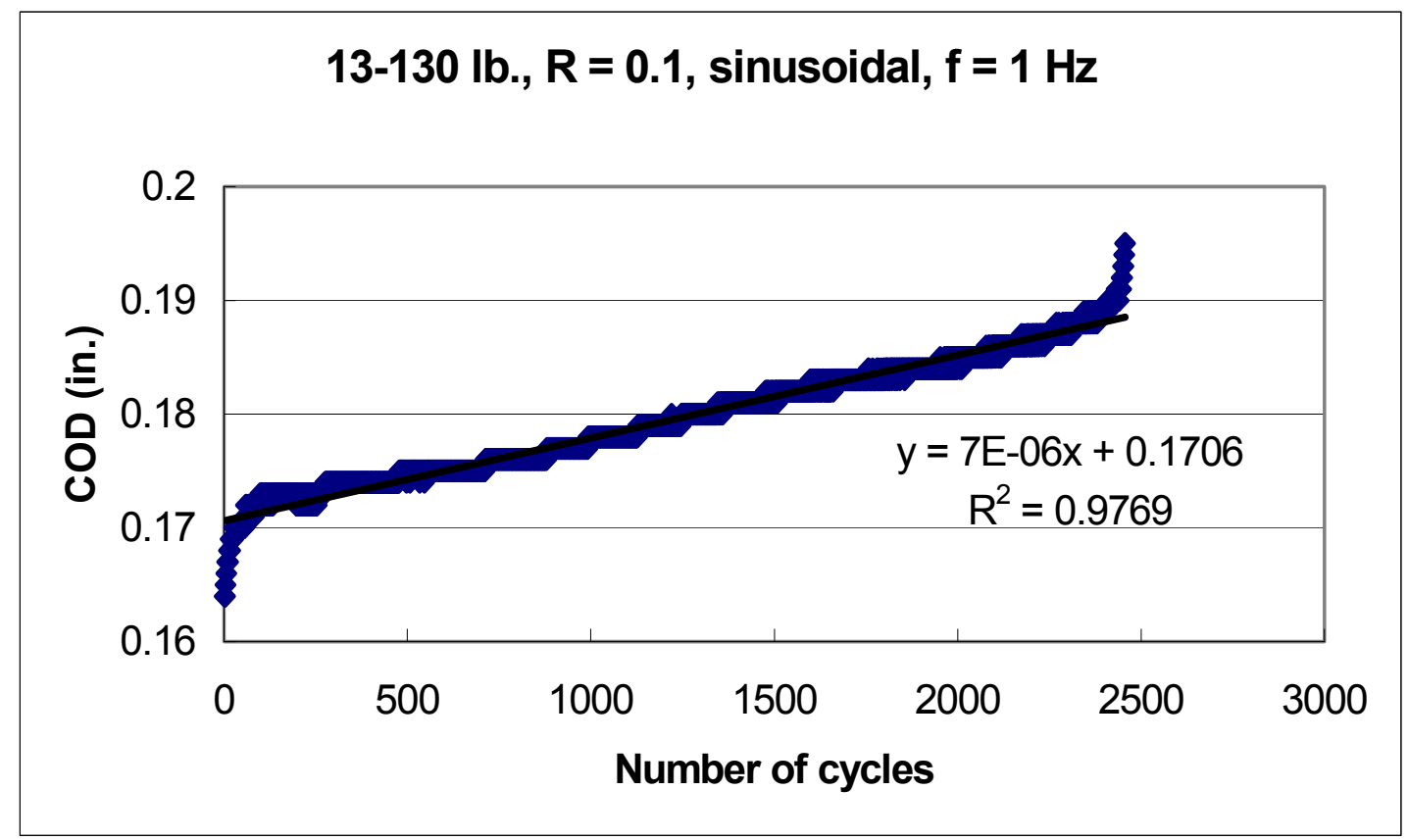

Fig. 3.10 COD versus number of cycles for a CDCB specimen under 13-130 lb. cyclic loading with a frequency of $1 \mathrm{~Hz}$, load ratio $R=0.1$ and sinusoidal waveform 


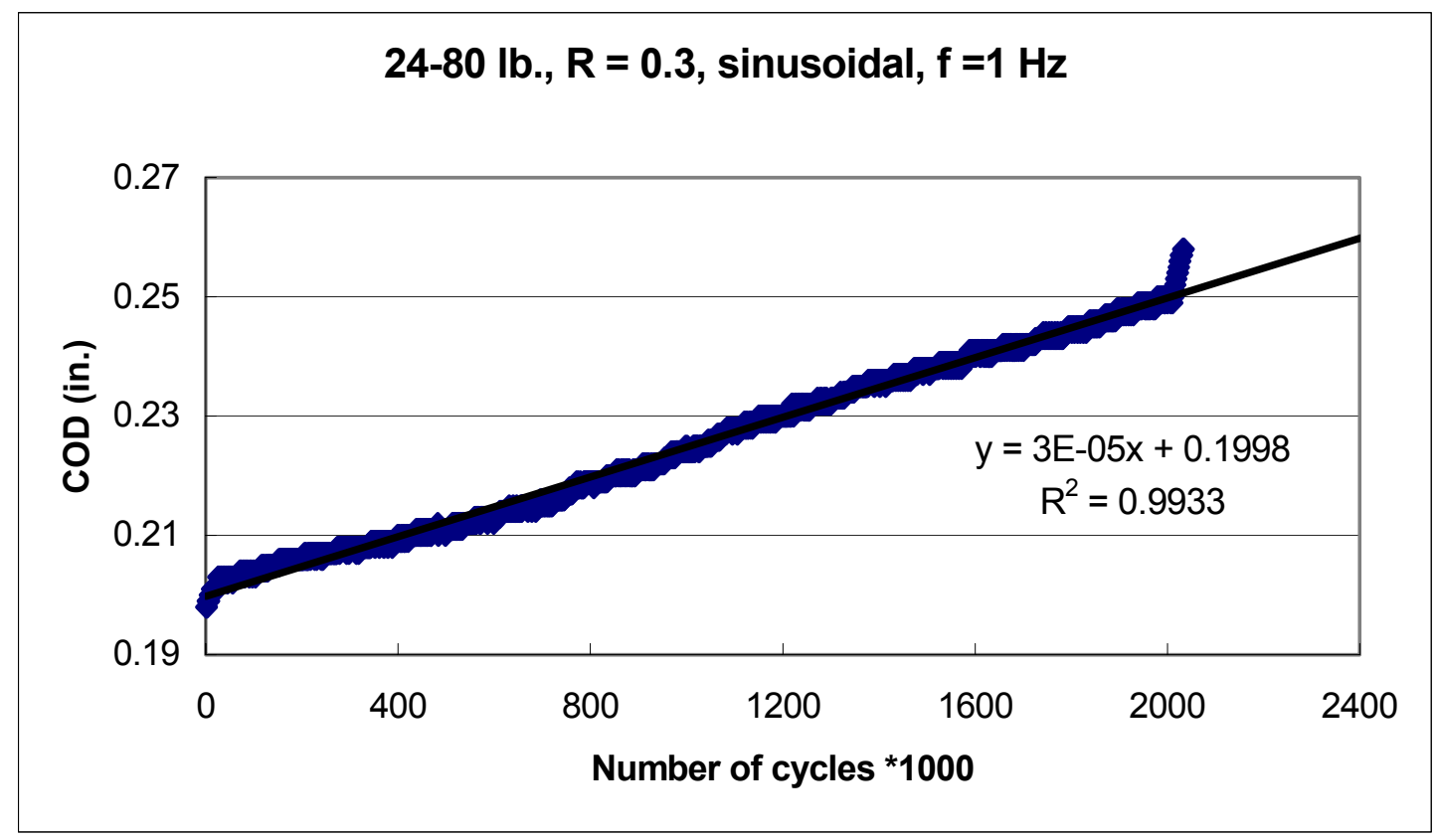

Fig. 3.11 COD versus number of cycles for a CDCB specimen under 24-80 lb. cyclic loading with a frequency of $1 \mathrm{~Hz}$, load ratio $R=0.3$ and sinusoidal waveform 


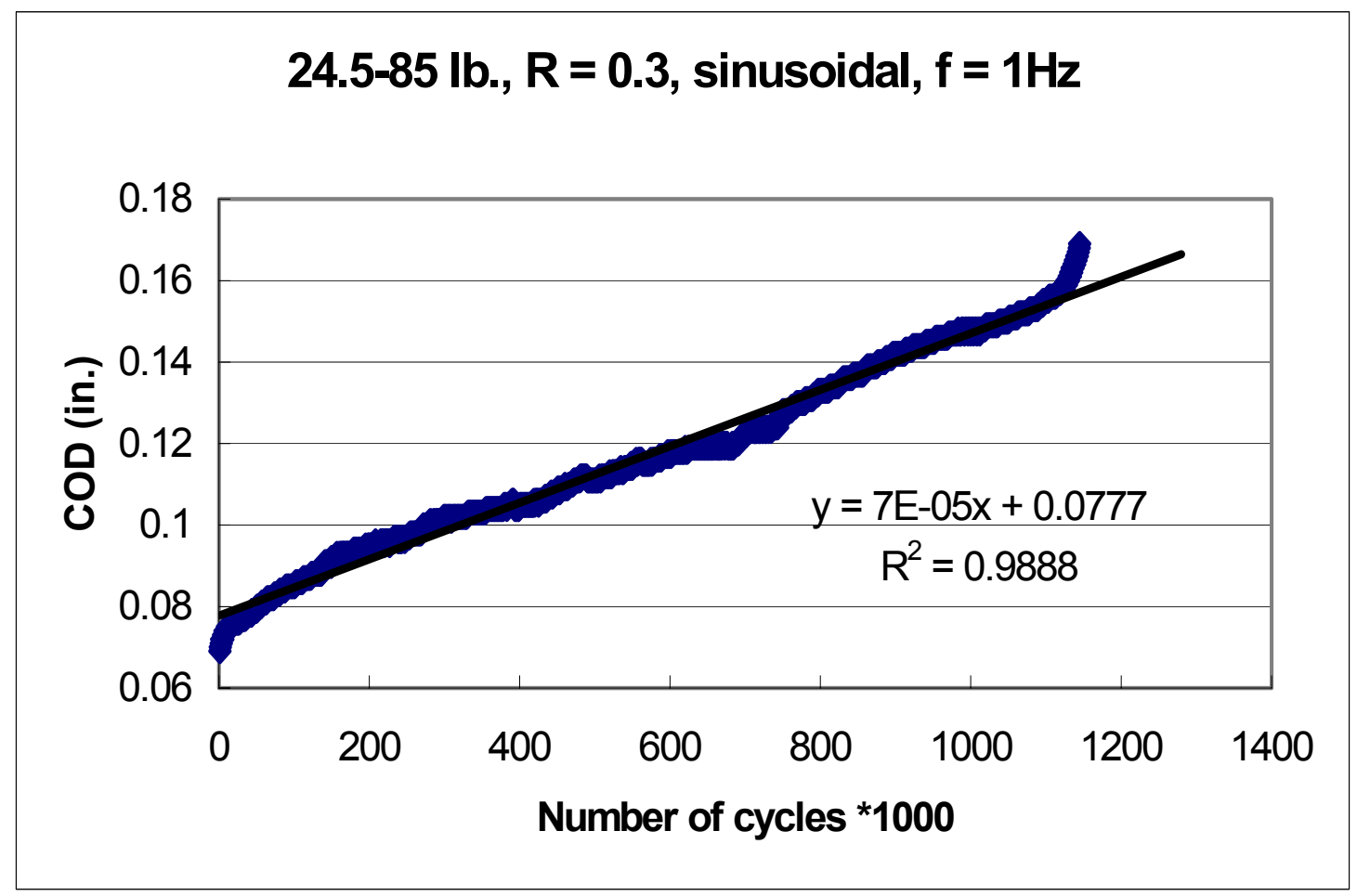

Fig. 3.12 COD versus number of cycles for a CDCB specimen under 24.5-85 lb. cyclic loading with a frequency of $1 \mathrm{~Hz}$, load ratio $R=0.3$ and sinusoidal waveform 


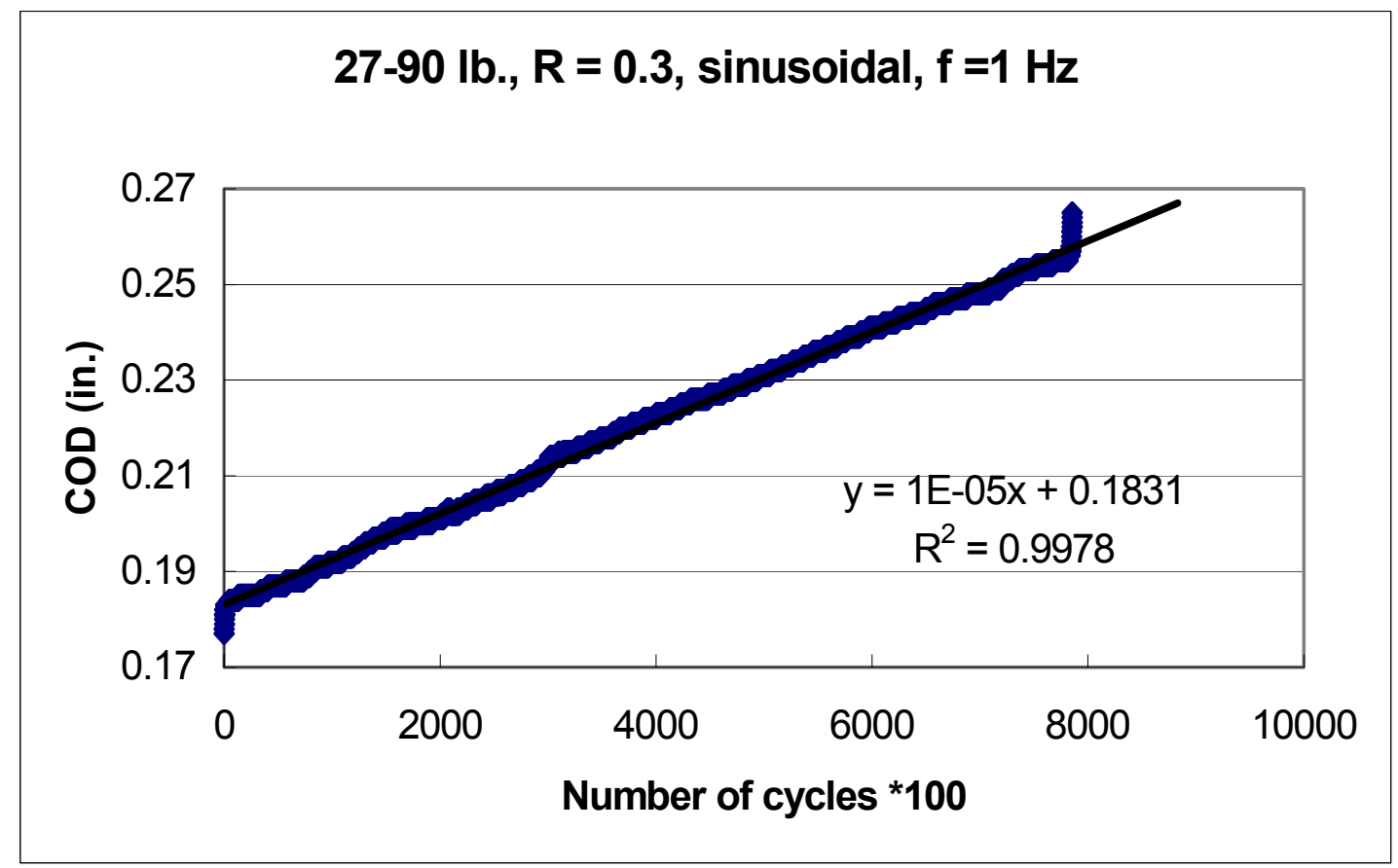

Fig. 3.13 COD versus number of cycles for a CDCB specimen under 27-90 lb. cyclic loading with a frequency of $1 \mathrm{~Hz}$, load ratio $R=0.3$ and sinusoidal waveform 


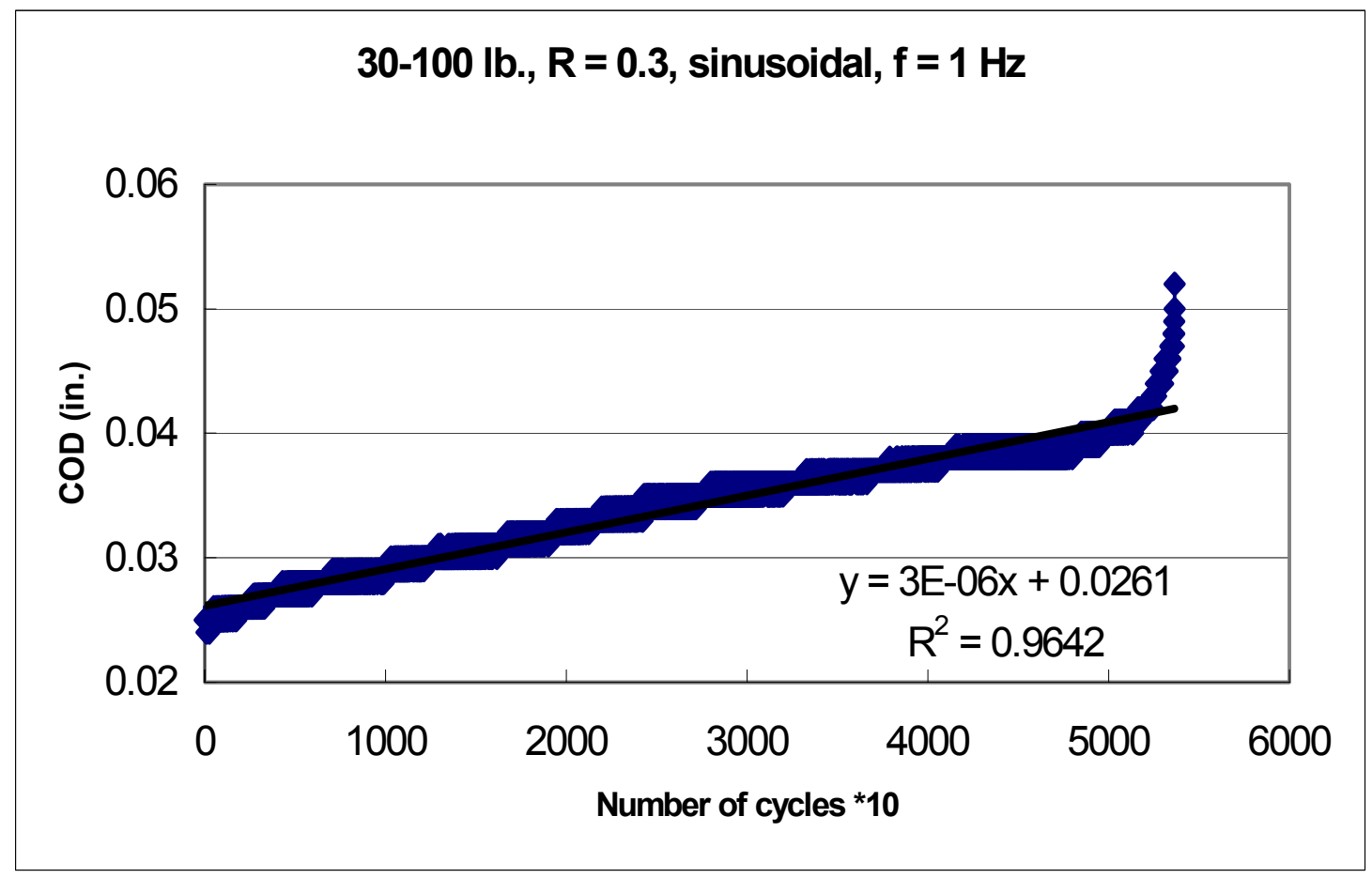

Fig. 3.14 COD versus number of cycles for a CDCB specimen under 30-100 lb. cyclic loading with a frequency of $1 \mathrm{~Hz}$, load ratio $R=0.3$ and sinusoidal waveform 


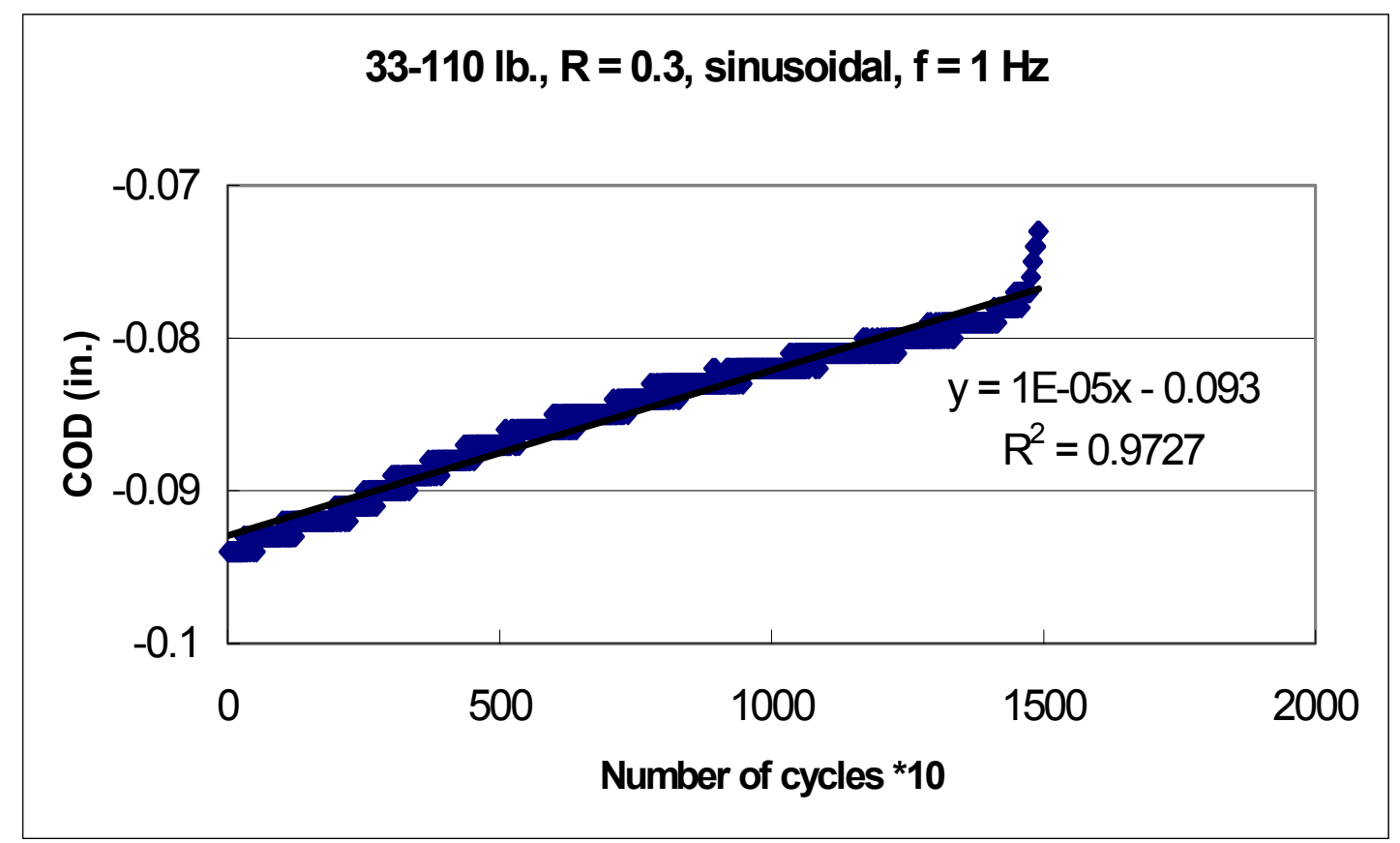

Fig. 3.15 COD versus number of cycles for a CDCB specimen under 33-110 lb. cyclic loading with a frequency of $1 \mathrm{~Hz}$, load ratio $R=0.3$ and sinusoidal waveform 


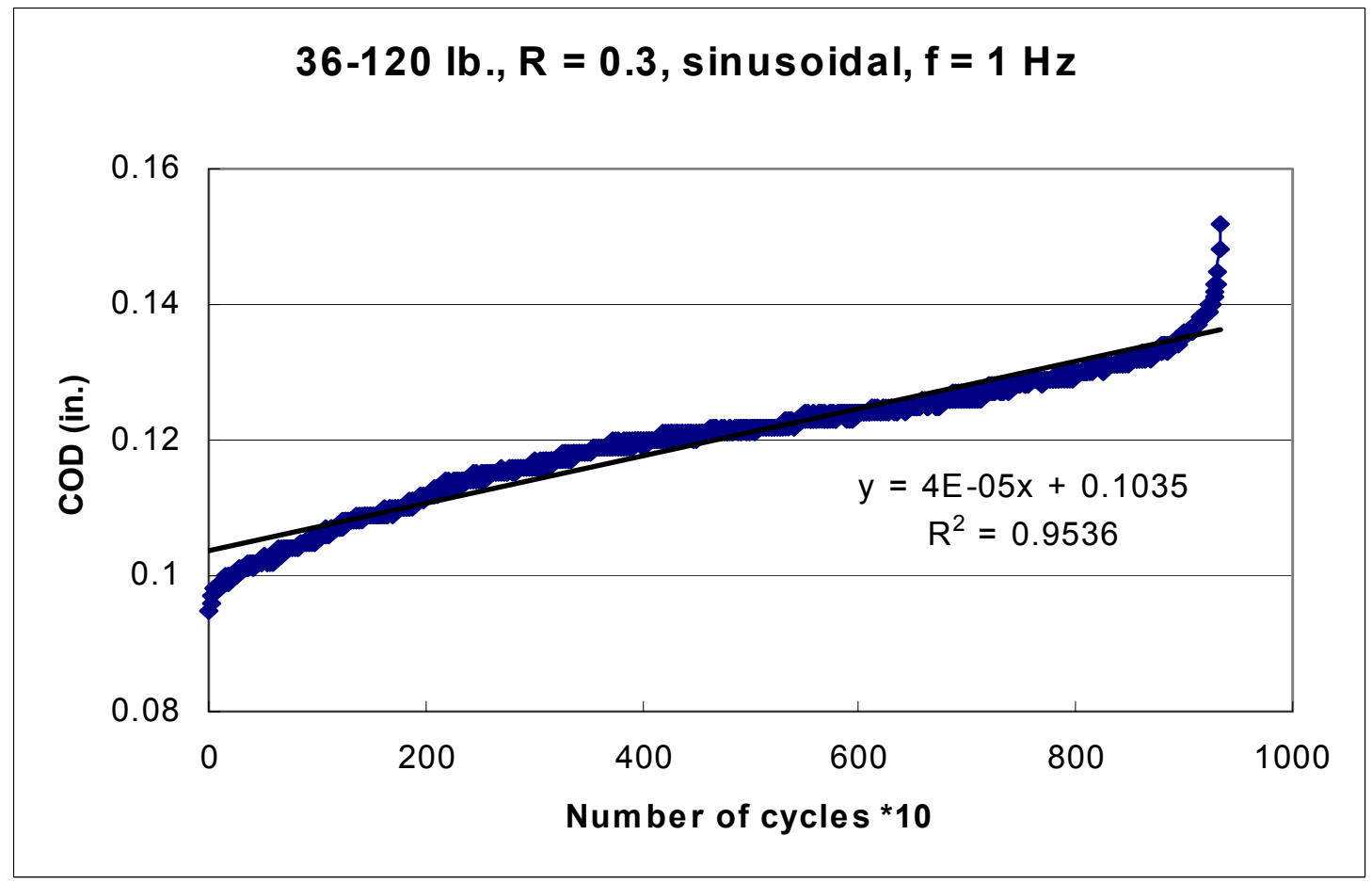

Fig. 3.16 COD versus number of cycles for a CDCB specimen under 36-120 lb. cyclic loading with a frequency of $1 \mathrm{~Hz}$, load ratio $R=0.3$ and sinusoidal waveform 


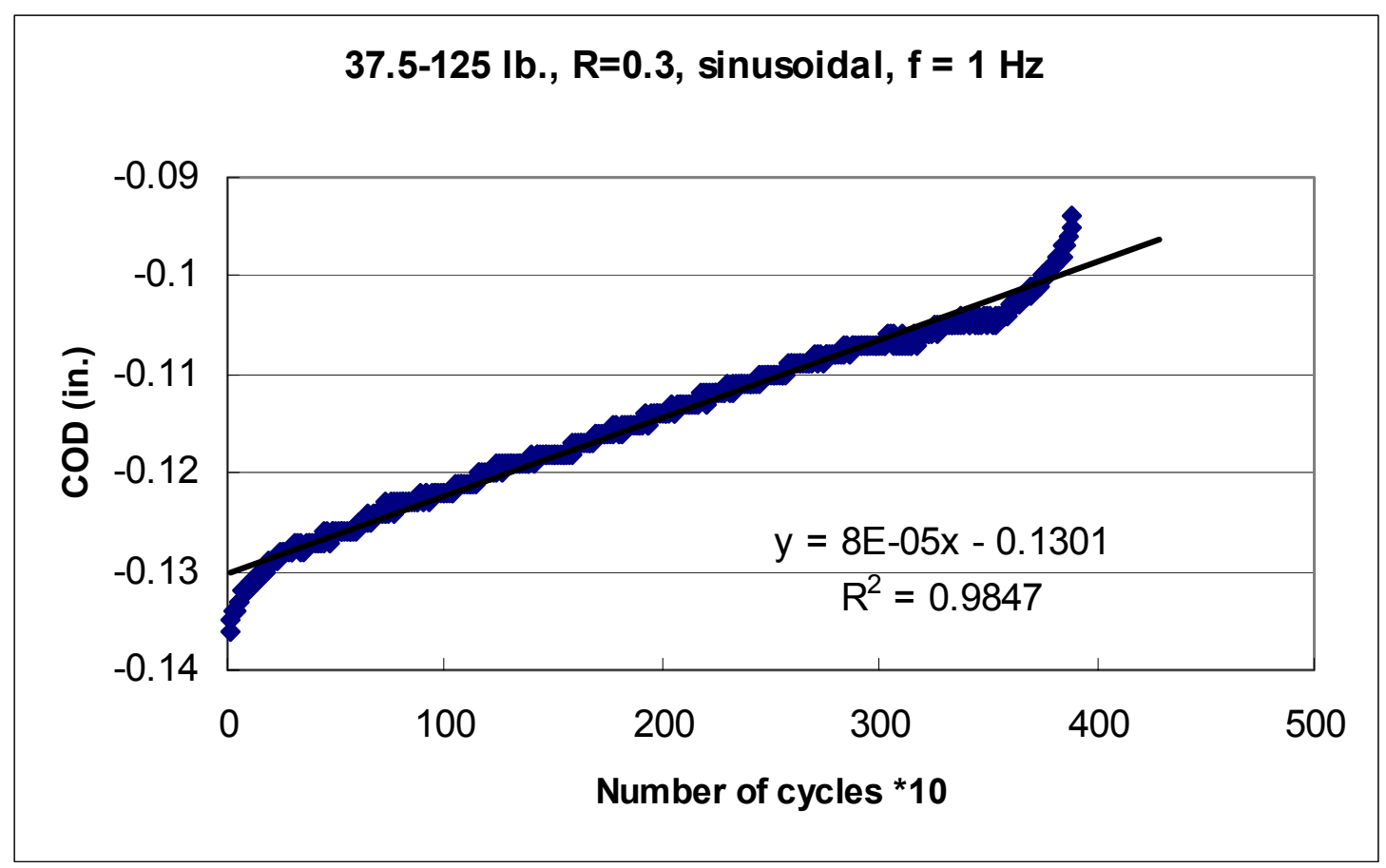

Fig. 3.17 COD versus number of cycles for a CDCB specimen under 37.5-125 lb. cyclic loading with a $1 \mathrm{~Hz}$, load ratio $\mathrm{R}=0.3$ and sinusoidal waveform 


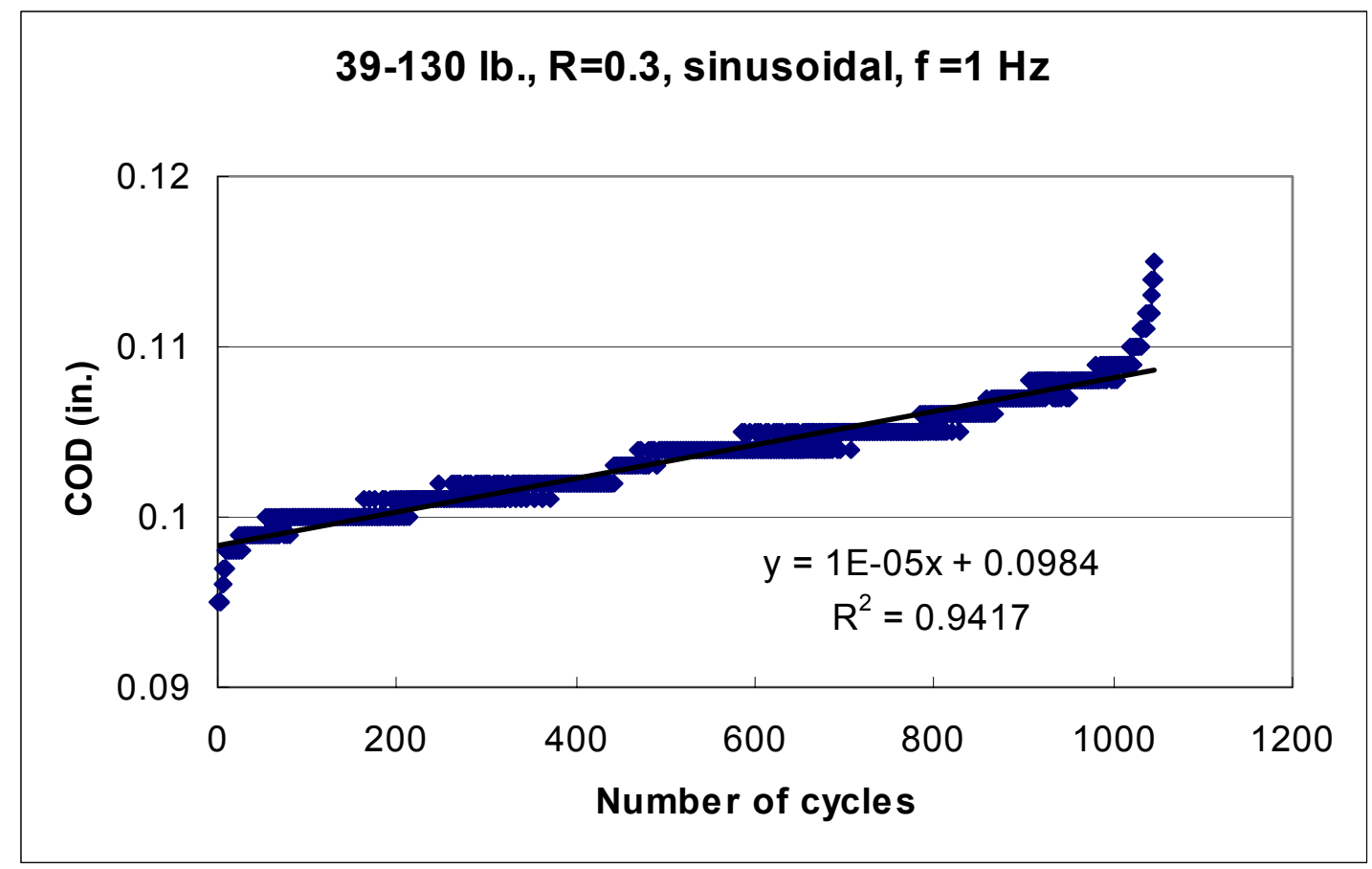

Fig. 3.18 COD versus number of cycles for a CDCB specimen under 39-130 lb. cyclic loading with a frequency of $1 \mathrm{~Hz}$, load ratio $R=0.3$ and sinusoidal waveform 


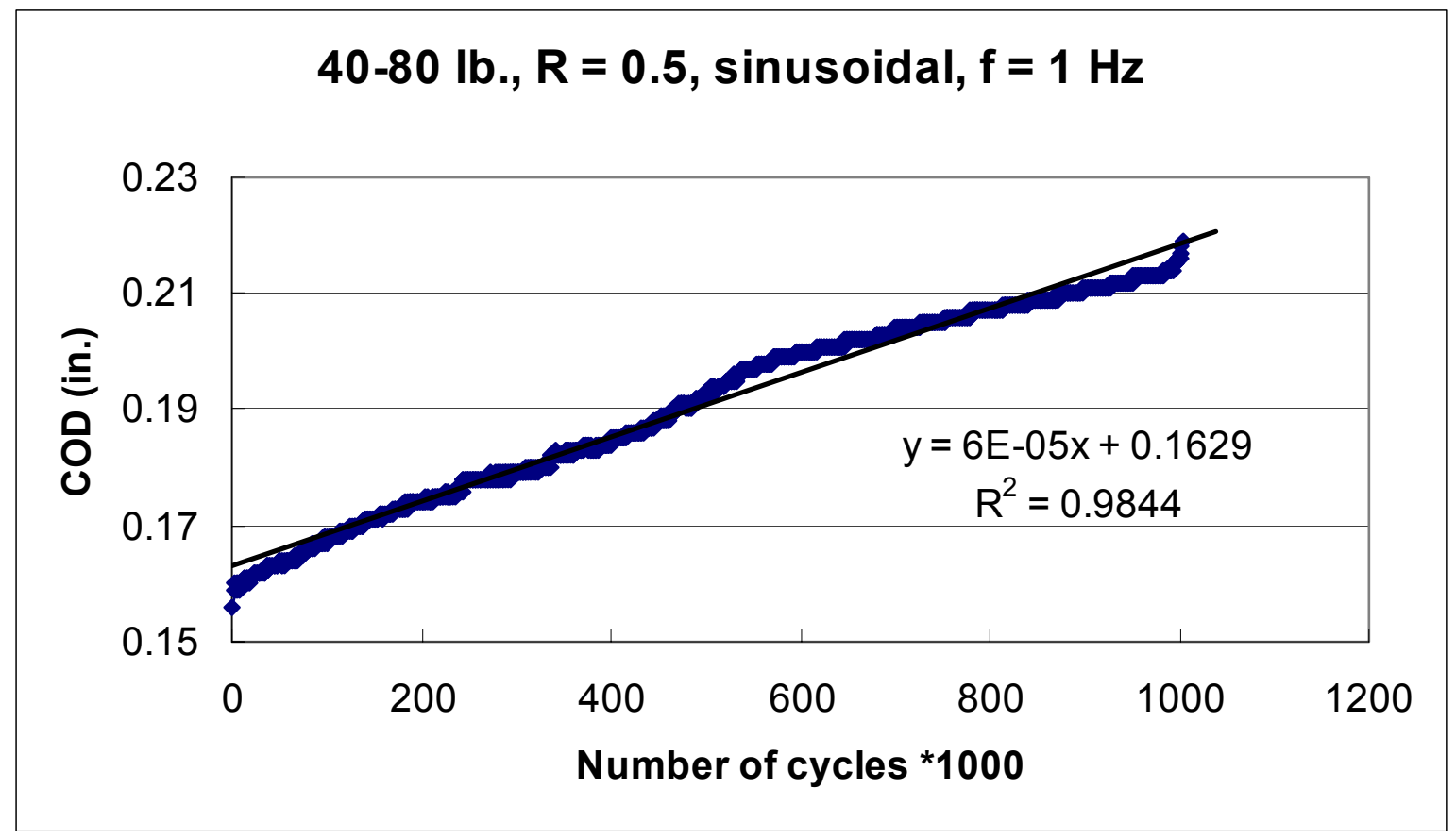

Fig. 3.19 COD versus number of cycles for a CDCB specimen under 40-80 lb. cyclic loading with a frequency of $1 \mathrm{~Hz}$, load ratio $R=0.5$ and sinusoidal waveform 


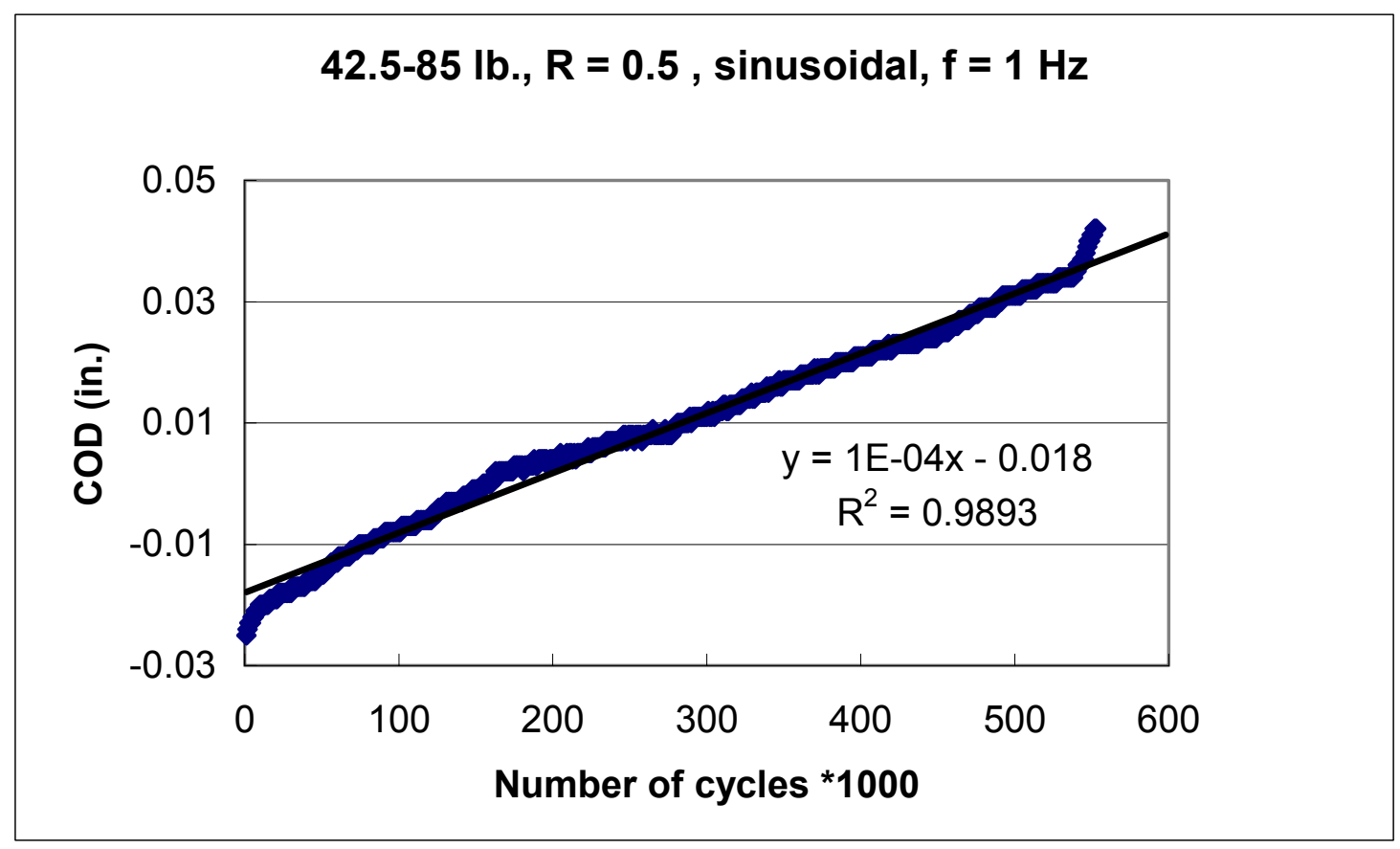

Fig. 3.20 COD versus number of cycles for a CDCB specimen under 42.5-85 lb. cyclic loading with a frequency of $1 \mathrm{~Hz}$, load ratio $R=0.5$ and sinusoidal waveform 


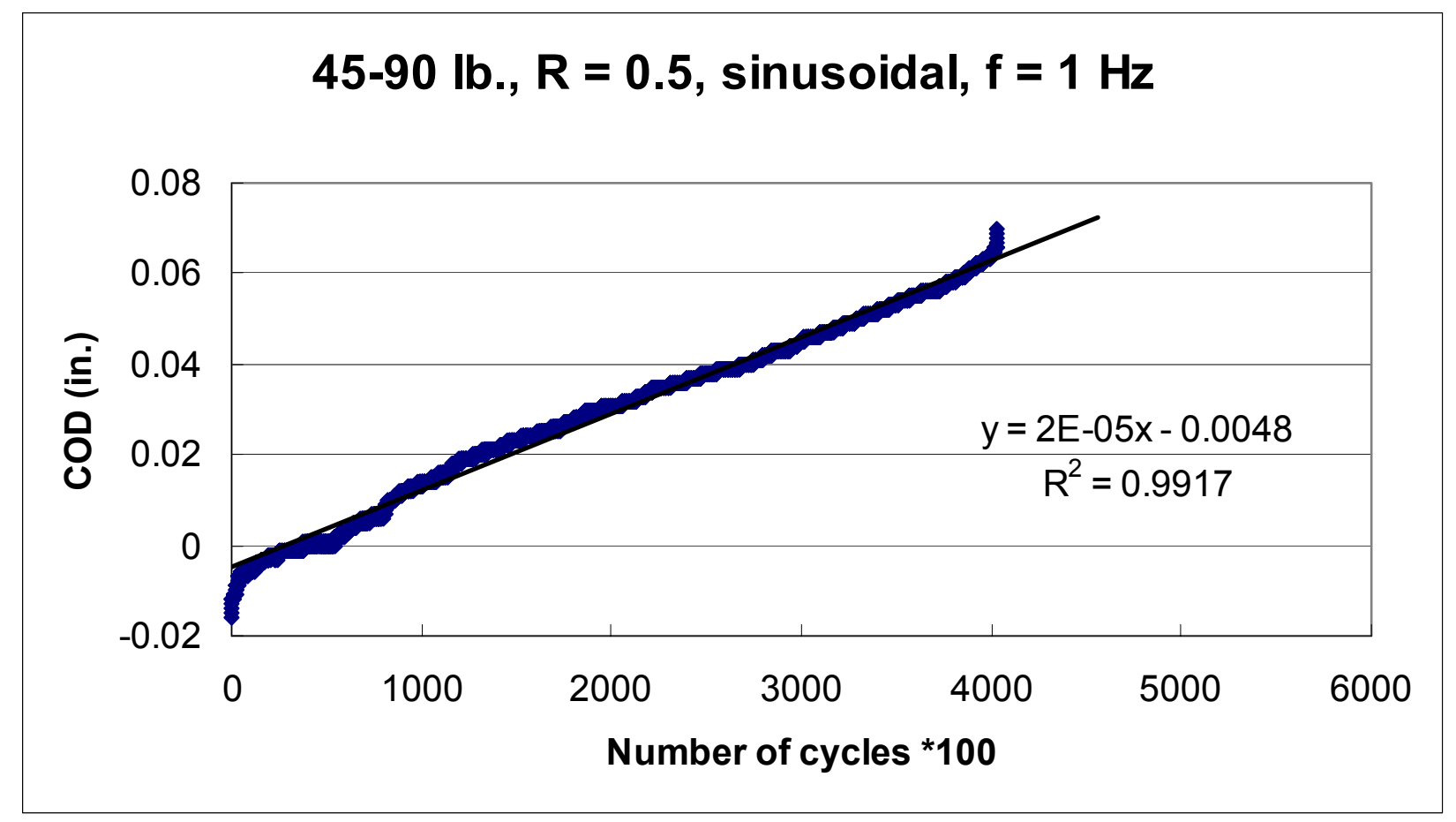

Fig. 3.21 COD versus number of cycles for a CDCB specimen under 45-90 lb. cyclic loading with a frequency of $1 \mathrm{~Hz}$, load ratio $R=0.5$ and sinusoidal waveform 


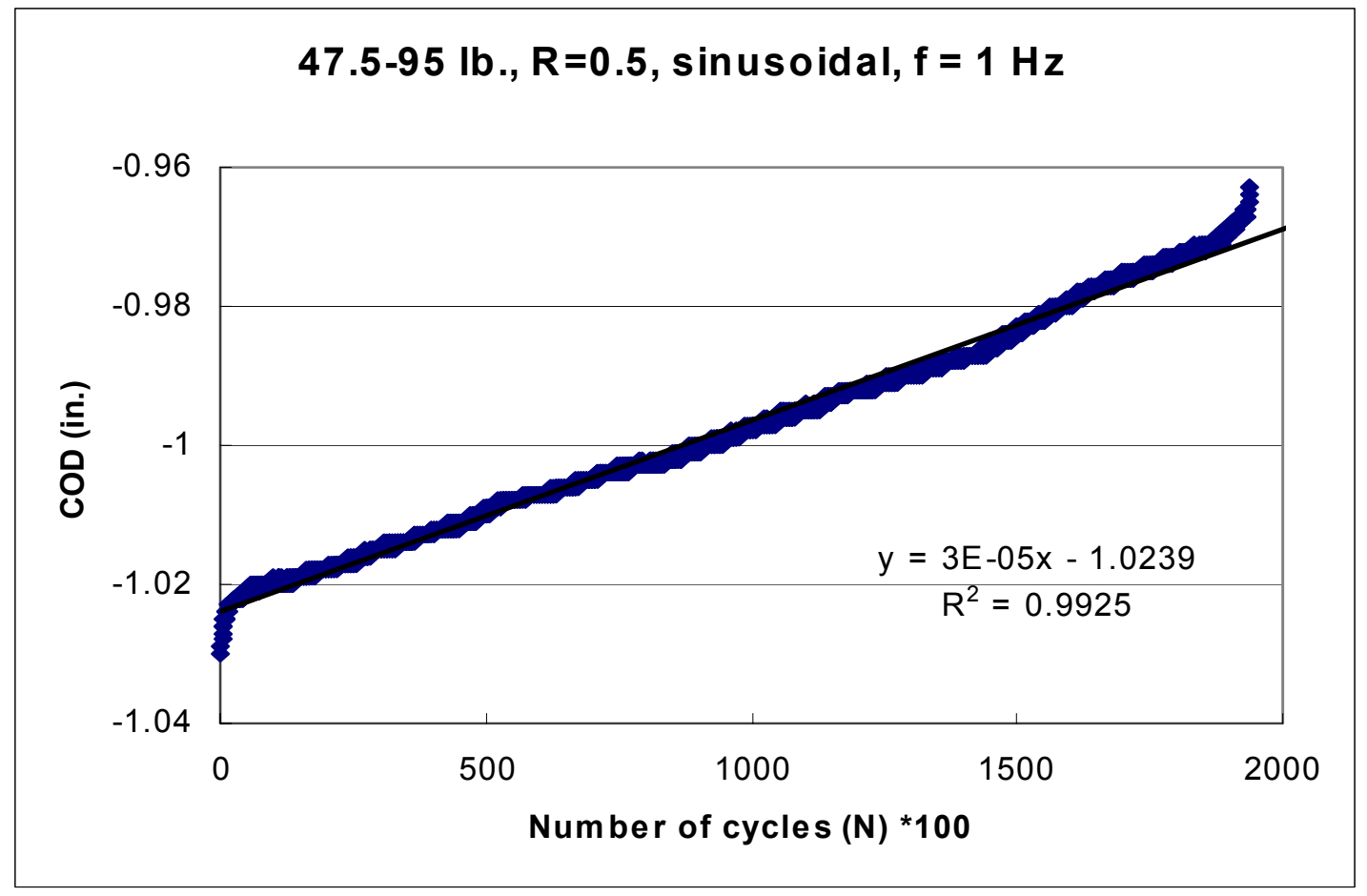

Fig. 3.22 COD versus number of cycles for a CDCB specimen under 47.5-95 lb. cyclic loading with a frequency of $1 \mathrm{~Hz}$, load ratio $R=0.5$ and sinusoidal waveform 


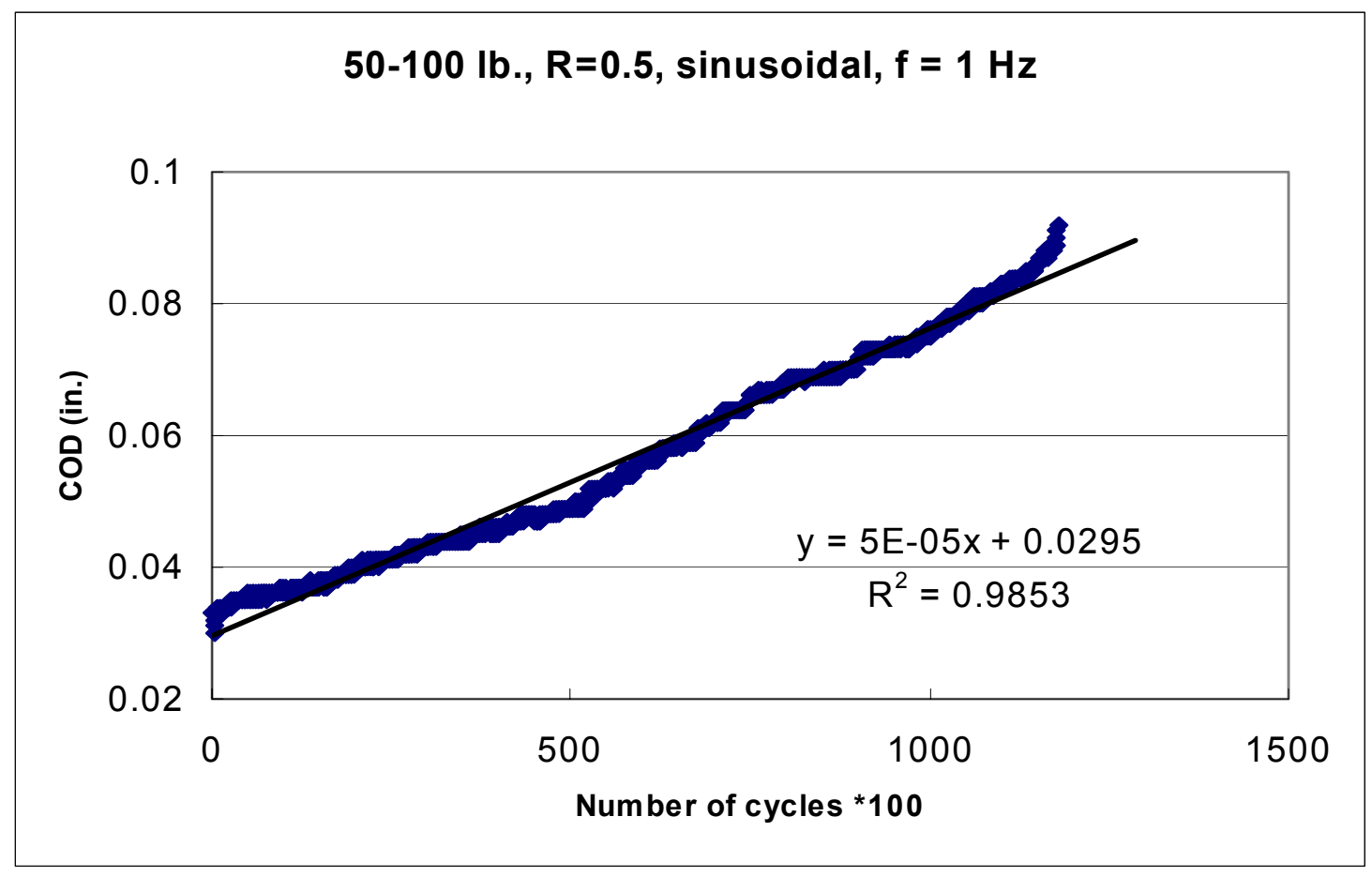

Fig. 3.23 COD versus number of cycles for a CDCB specimen under 50-100 lb. cyclic loading with a frequency of $1 \mathrm{~Hz}$, load ratio $R=0.5$ and sinusoidal waveform 


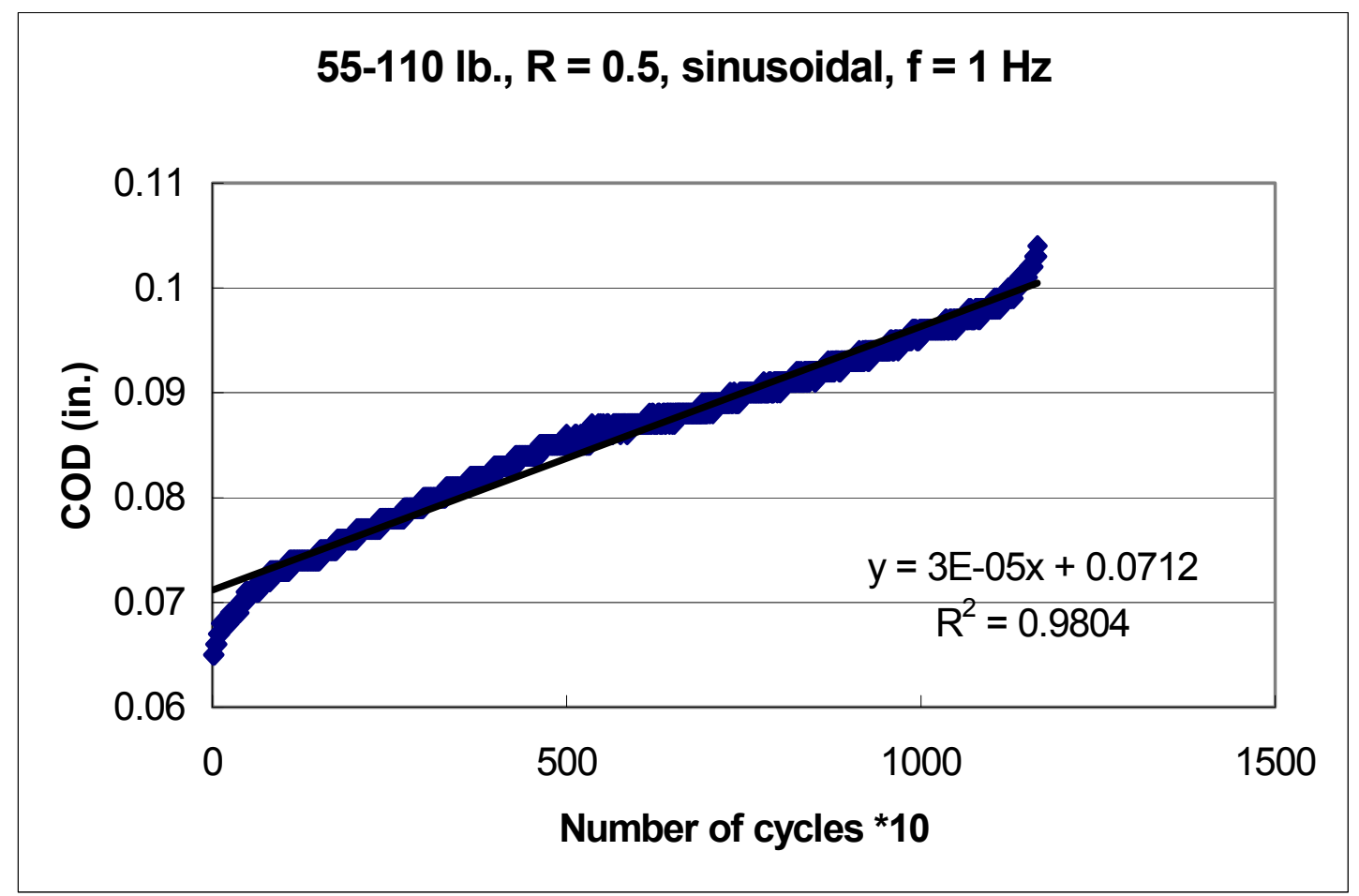

Fig. 3.24 COD versus number of cycles for a CDCB specimen under 55-110 lb. cyclic loading with a frequency of $1 \mathrm{~Hz}$, load ratio $R=0.5$ and sinusoidal waveform 


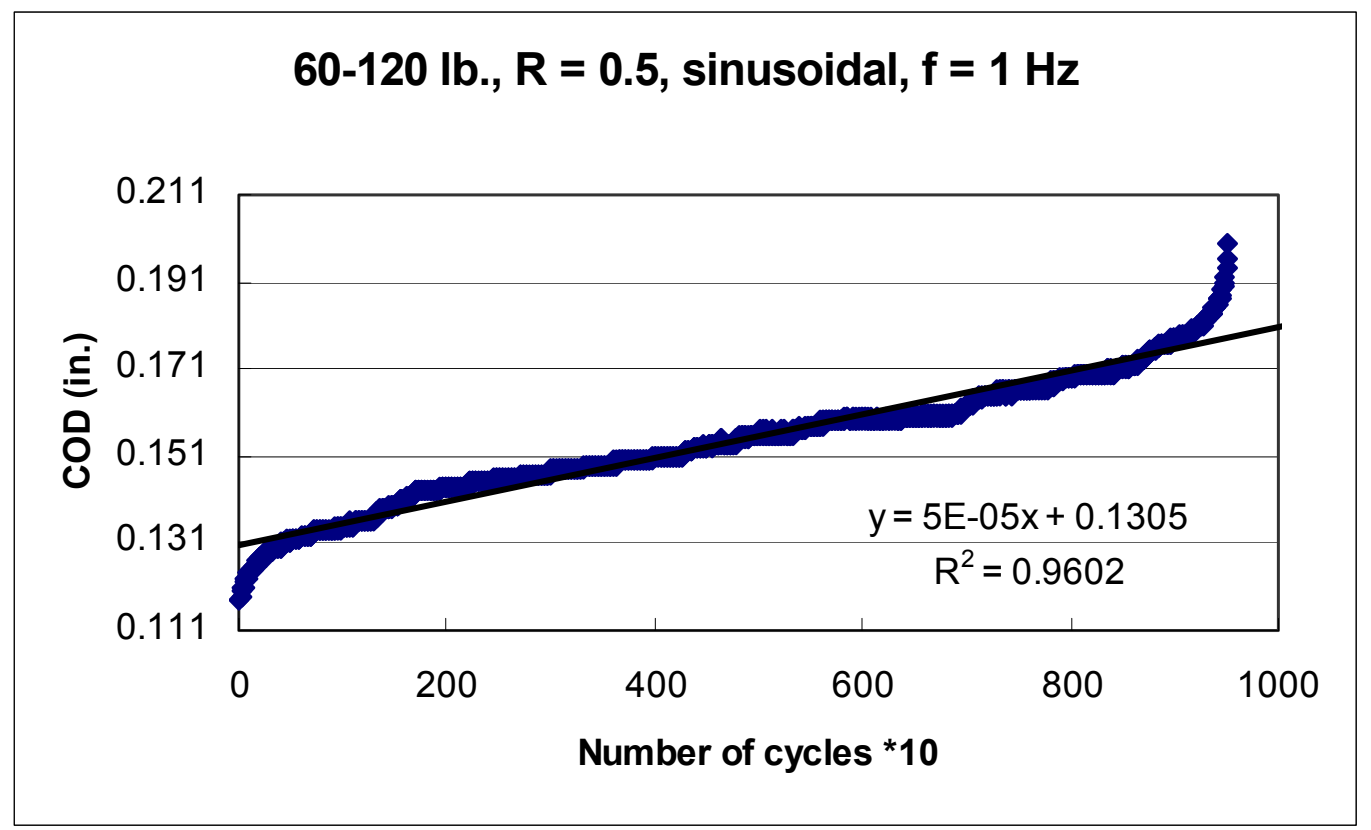

Fig. 3.25 COD versus number of cycles for a CDCB specimen under 60-120 lb. cyclic loading with a frequency of $1 \mathrm{~Hz}$, load ratio $R=0.5$ and sinusoidal waveform 


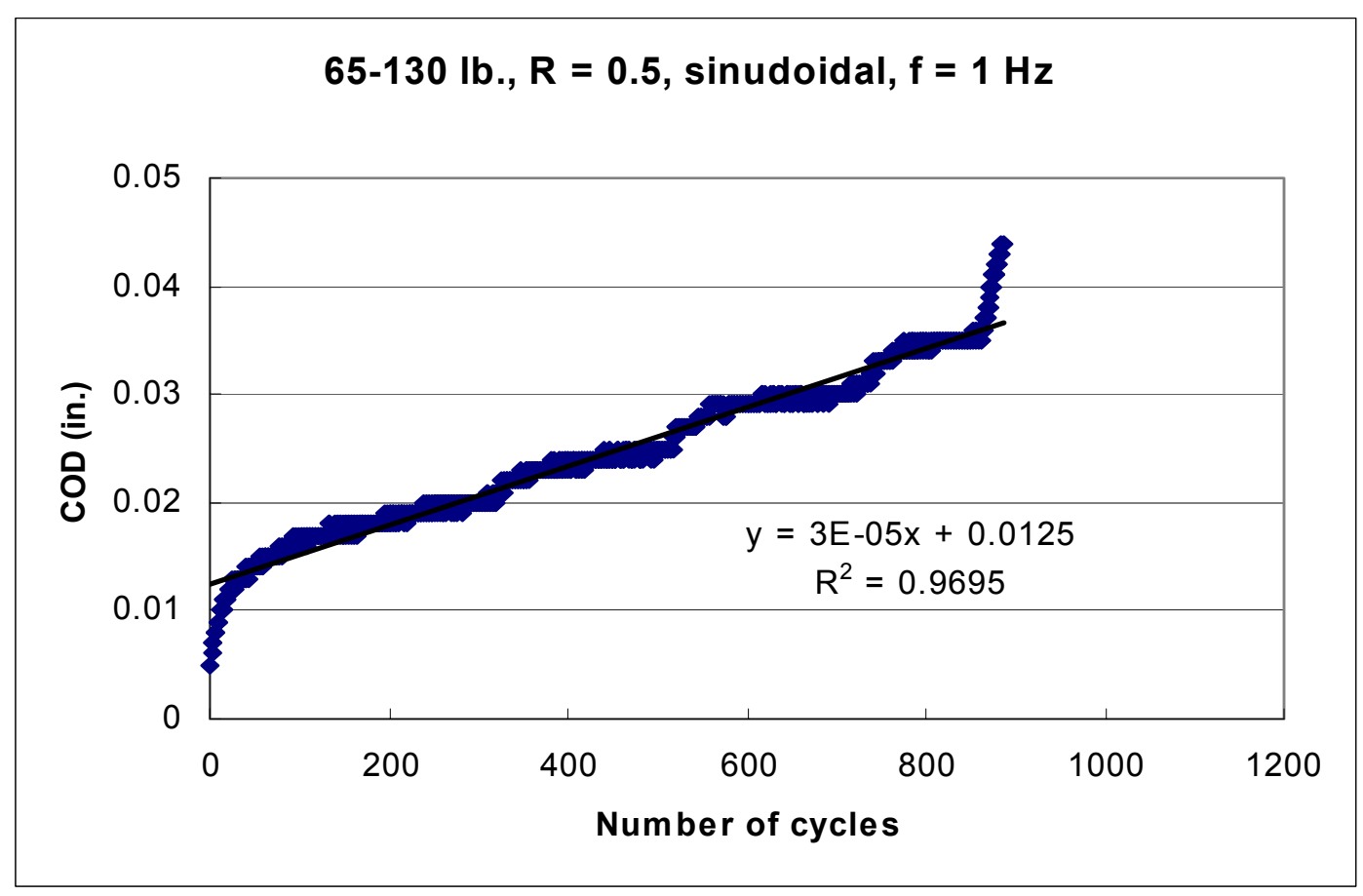

Fig. 3.26 COD versus number of cycles for a CDCB specimen under 65-130 lb. cyclic loading with a frequency of $1 \mathrm{~Hz}$, load ratio $R=0.5$ and sinusoidal waveform 
Table 3.5 Experimental results of the COD propagation rate for the load ratio $R=0.1$ under a constant frequency $f=1 \mathrm{~Hz}$ and sinusoidal waveform

\begin{tabular}{ccc}
\hline$P_{\max }(\mathrm{lb})$. & $P_{\min }(\mathrm{lb})$. & $d C O D / d N($ in./cycle $)$ \\
130 & 13 & $7.00 \mathrm{E}-06$ \\
120 & 12 & $2.00 \mathrm{E}-06$ \\
110 & 11 & $6.00 \mathrm{E}-07$ \\
100 & 10 & $1.00 \mathrm{E}-07$ \\
90 & 9 & $7.00 \mathrm{E}-08$ \\
85 & 8.5 & $4.00 \mathrm{E}-08$ \\
80 & 8 & $2.00 \mathrm{E}-08$ \\
\hline
\end{tabular}

Table 3.6 Experimental results of the COD propagation rate for the load ratio $R=0.3$ under a constant frequency $f=1 \mathrm{~Hz}$ and sinusoidal waveform

\begin{tabular}{ccc}
\hline$P_{\max }(\mathrm{lb})$. & $P_{\min }(\mathrm{lb})$. & $d C O D / d N($ in./cycle $)$ \\
130 & 39 & $1.00 \mathrm{E}-05$ \\
125 & 37.5 & $8.00 \mathrm{E}-06$ \\
120 & 36 & $4.00 \mathrm{E}-06$ \\
110 & 33 & $1.00 \mathrm{E}-06$ \\
100 & 30 & $3.00 \mathrm{E}-07$ \\
90 & 27 & $1.00 \mathrm{E}-07$ \\
85 & 25.5 & $7.00 \mathrm{E}-08$ \\
80 & 24 & $3.00 \mathrm{E}-08$ \\
\hline
\end{tabular}


Table 3.7 Experimental results of the COD propagation rate for the load ratio $R=0.5$ under a constant frequency $f=1 \mathrm{~Hz}$ and sinusoidal waveform

\begin{tabular}{ccc}
\hline$P_{\max }(\mathrm{lb})$. & $P_{\min }(\mathrm{lb})$. & $d C O D / d N($ in./cycle $)$ \\
130 & 65 & $3.00 \mathrm{E}-05$ \\
120 & 60 & $5.00 \mathrm{E}-06$ \\
110 & 55 & $3.00 \mathrm{E}-06$ \\
100 & 50 & $5.00 \mathrm{E}-07$ \\
95 & 47.5 & $3.00 \mathrm{E}-07$ \\
90 & 45 & $2.00 \mathrm{E}-07$ \\
85 & 42.5 & $1.00 \mathrm{E}-07$ \\
80 & 40 & $6.00 \mathrm{E}-08$ \\
\hline
\end{tabular}

\subsubsection{Crack growth rate determination by compliance method}

The accuracy of crack propagation rate $d a / d N$ depends on the measurement of crack length, which is a formidable problem confronted by researchers of fracture mechanics. The crack opening displacement (COD) is much easier to measure than the crack length for the CDCB specimen. The difficulty of measuring crack propagation rate is avoided by recording instead COD propagation rate. The transformation from crack opening displacement propagation rate $d C O D / d N$ to crack propagation rate $d a / d N$ is given as

$$
\frac{d a}{d N}=\frac{d a}{d C} \frac{d C}{d N}=\frac{1}{k P} \frac{d C O D}{d N}
$$


where $N$ is the number of cycles, $P$ is the applied load, and $k$ is a constant that is equal to the $d C / d a$ value of the CDCB specimen. A typical crack opening displacement propagation rate diagram from fatigue testing is illustrated in figure 3.3. Tables 3.8, 3.9, and 3.10 show the crack propagation rate $d a / d N$ obtained from experimental data of $d C O D / d N$ by using Eq. (3.20).

Table 3.8 The crack propagation rate $d a / d N$ for load ratio $R=0.1$

\begin{tabular}{ccccc}
\hline$G_{\max }(\mathrm{lb} /$ in. $)$ & $G_{\min }(\mathrm{lb} . / \mathrm{in})$. & $\Delta G(\mathrm{lb} . / \mathrm{in})$. & $d C O D / d N($ in./cycle $)$ & $d a / d N($ in. $/$ cycle $)$ \\
\hline 2.00 & 0.02 & 1.98 & $7.00 \mathrm{E}-06$ & $1.82 \mathrm{E}-04$ \\
1.70 & 0.02 & 1.68 & $2.00 \mathrm{E}-06$ & $5.63 \mathrm{E}-05$ \\
1.43 & 0.01 & 1.42 & $6.00 \mathrm{E}-07$ & $1.84 \mathrm{E}-05$ \\
1.18 & 0.01 & 1.17 & $1.00 \mathrm{E}-07$ & $1.01 \mathrm{E}-05$ \\
0.96 & 0.01 & 0.95 & $7.00 \mathrm{E}-08$ & $2.63 \mathrm{E}-06$ \\
0.86 & 0.01 & 0.85 & $4.00 \mathrm{E}-08$ & $1.59 \mathrm{E}-06$ \\
0.76 & 0.01 & 0.75 & $2.00 \mathrm{E}-08$ & $8.45 \mathrm{E}-07$ \\
\hline
\end{tabular}

Table 3.9 The crack propagation rate $d a / d N$ for load ratio $R=0.3$

\begin{tabular}{ccccc}
\hline$G_{\max }(\mathrm{lb} . / \mathrm{in})$. & $G_{\min }(\mathrm{lb} . / \mathrm{in})$. & $\Delta G(\mathrm{lb} . / \mathrm{in})$. & $d C O D / d N($ in./cycle $)$ & $d a / d N($ in./cycle $)$ \\
\hline 2.00 & 0.18 & 1.82 & $1.00 \mathrm{E}-05$ & $2.60 \mathrm{E}-04$ \\
1.85 & 0.17 & 1.68 & $8.00 \mathrm{E}-06$ & $2.16 \mathrm{E}-04$ \\
1.70 & 0.15 & 1.55 & $4.00 \mathrm{E}-06$ & $1.13 \mathrm{E}-04$ \\
1.43 & 0.13 & 1.30 & $1.00 \mathrm{E}-06$ & $3.07 \mathrm{E}-05$ \\
1.18 & 0.11 & 1.07 & $3.00 \mathrm{E}-07$ & $1.01 \mathrm{E}-05$ \\
0.96 & 0.09 & 0.87 & $1.00 \mathrm{E}-07$ & $3.76 \mathrm{E}-06$ \\
0.86 & 0.08 & 0.78 & $7.00 \mathrm{E}-08$ & $2.78 \mathrm{E}-06$ \\
0.76 & 0.07 & 0.69 & $3.00 \mathrm{E}-08$ & $1.27 \mathrm{E}-06$ \\
\hline
\end{tabular}


Table 3.10 The crack propagation rate $d a / d N$ for load ratio $R=0.5$

\begin{tabular}{ccccc}
\hline$G_{\max }(\mathrm{lb} . / \mathrm{in})$. & $G_{\min }(\mathrm{lb} . / \mathrm{in})$. & $\Delta G(\mathrm{lb} . / \mathrm{in})$. & $d C O D / d N($ in./cycle $)$ & $d a / d N($ in./cycle $)$ \\
\hline 2.00 & 0.50 & 1.50 & $3.00 \mathrm{E}-05$ & $7.80 \mathrm{E}-04$ \\
1.70 & 0.43 & 1.27 & $5.00 \mathrm{E}-06$ & $1.41 \mathrm{E}-04$ \\
1.43 & 0.36 & 1.07 & $3.00 \mathrm{E}-06$ & $9.22 \mathrm{E}-05$ \\
1.18 & 0.30 & 0.88 & $5.00 \mathrm{E}-07$ & $1.69 \mathrm{E}-05$ \\
1.07 & 0.27 & 0.80 & $3.00 \mathrm{E}-07$ & $1.07 \mathrm{E}-05$ \\
0.96 & 0.24 & 0.72 & $2.00 \mathrm{E}-07$ & $7.51 \mathrm{E}-06$ \\
0.86 & 0.21 & 0.65 & $1.00 \mathrm{E}-07$ & $3.98 \mathrm{E}-06$ \\
0.76 & 0.19 & 0.57 & $6.00 \mathrm{E}-08$ & $2.53 \mathrm{E}-06$ \\
\hline
\end{tabular}

\subsubsection{Constant strain energy release rate range calculation}

The contoured double cantilever beam (CDCB) specimen was used for evaluating fracture toughness of bonded wood-FRP interfaces under mode-I loading. The strain energy release rate $G$ is given as

$$
G=\frac{P^{2}}{2 b} \frac{d C}{d a}
$$

where, $P=$ the applied load; $b=$ the thickness of the specimen; $C=$ the compliance; and $a$ $=$ the crack length. For one complete cycle (see Fig. 2.7), the maximum and minimum 
strain energy release rates are obtained simply by substituting the maximum load and minimum load into Eq. (3.21). They are given as

$$
\begin{aligned}
G_{\max } & =\frac{P_{\max }^{2}}{2 b} \frac{d C}{d a} \\
G_{\min } & =\frac{P_{\min }^{2}}{2 b} \frac{d C}{d a}
\end{aligned}
$$

The strain energy release rate range $\Delta G$ is easily obtained from the difference of equations (3.22) and (3.23). For the CDCB specimen, the rate of change of compliance with respect to crack length $d C / d a$ is a constant, and therefore, the constant strain energy release rate range $\Delta G$ is acquired for constant amplitude cyclic loading.

\subsubsection{A modified Paris Law model}

The relationship of crack propagation rate $d a / d N$ versus the strain energy release rate range $\Delta G$ for load ratios $R$ of $0.1,0.3$ and 0.5 is drawn respectively in Figs. 3.27, 3.28 and 3.29 from the corresponding data in Tables 3.8, 3.9 and 3.10. The strain energy release rate range $\Delta G$ itself includes the mean stress intensity factor effect or load ratio effect (Sutton, 1974). The modified Paris Law equation corresponding to the strain energy release rate range

$$
\frac{d a}{d N}=B(\Delta G)^{m}
$$


is used to fit the data points in Figs. 3.27, 3.28 and 3.29. For each load ratio $R$, the constants $B$ and $m$ are listed in Table 3.11, which indicates that both $B$ and $m$ increase as the load ratio $R$ increases. The log-log plot of crack propagation rate $d a / d N$ versus the strain energy release rate range $\Delta G$ is shown in Figure 3.30. It is easily seen that there is a strong effect of load ratio $R$ on crack propagation rate $d a / d N$. Crack propagation rate $d a / d N$ increases for the same strain energy release rate range, $\Delta G$, when load ratio $R$ increases. When $\Delta G$ is equal to about $0.8, d a / d N$ for load ratio $R=0.5$ increases about one order of magnitude compared with $d a / d N$ for load ratio $R=0.1$. For larger values of $\Delta G, d a / d N$ tends to increase even more. Again Eq. (3.24) is used to fit the experimental data of different load ratios. However, Eq. (3.24) is only valid for a specific load ratio $R$, and it fails to explain the load ratio effect for the experimental data. Therefore, it may be suitable for us to introduce another parameter to represent the load ratio effect.

Table 3.11 Material constants $B$ and $m$ in Eq. (3.24) for three load ratios $R$

\begin{tabular}{|c|c|c|}
\hline Load ratio $R$ & $B$ & $m$ \\
\hline 0.1 & $4 \times 10^{-6}$ & 5.37 \\
\hline 0.3 & $9 \times 10^{-6}$ & 5.59 \\
\hline 0.5 & $5 \times 10^{-5}$ & 5.77 \\
\hline
\end{tabular}




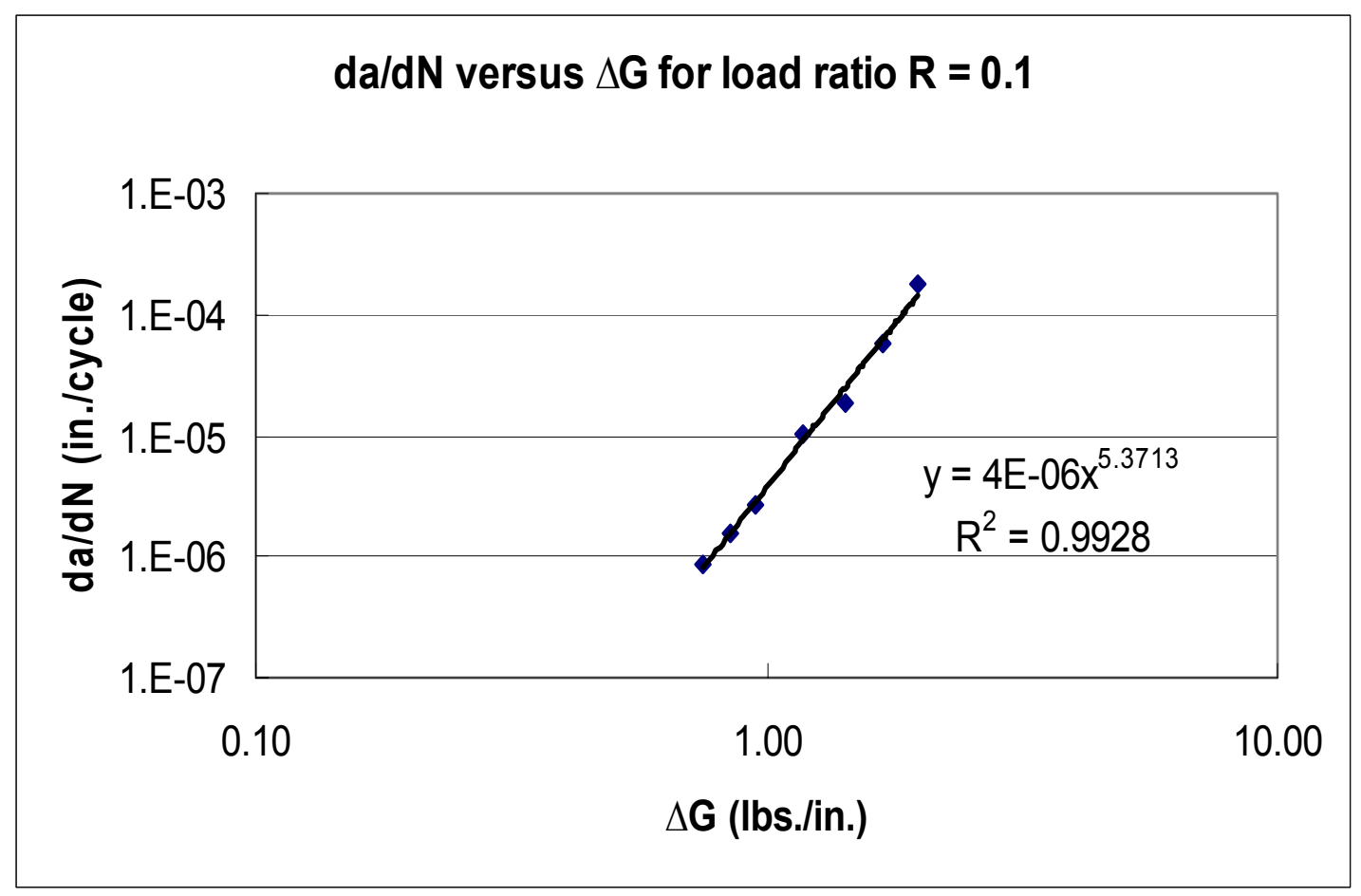

Fig. 3.27 The log-log plot of crack propagation rate $d a / d N$ versus the strain energy release rate range $\Delta G$ for load ratio $\mathrm{R}=0.1$ 


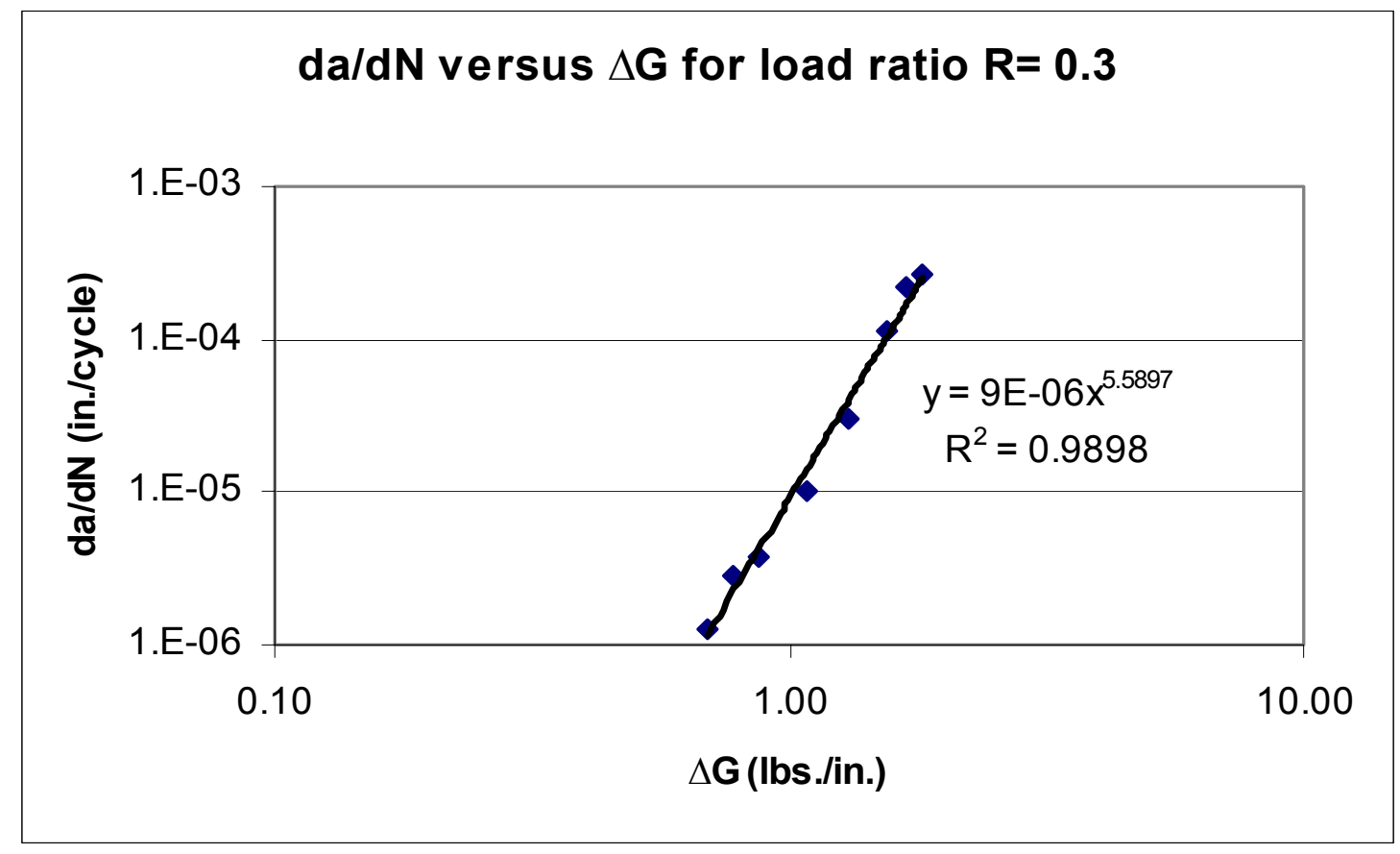

Fig. 3.28 The log-log plot of crack propagation rate $d a / d N$ versus the strain energy release rate range $\Delta G$ for load ratio $\mathrm{R}=0.3$ 


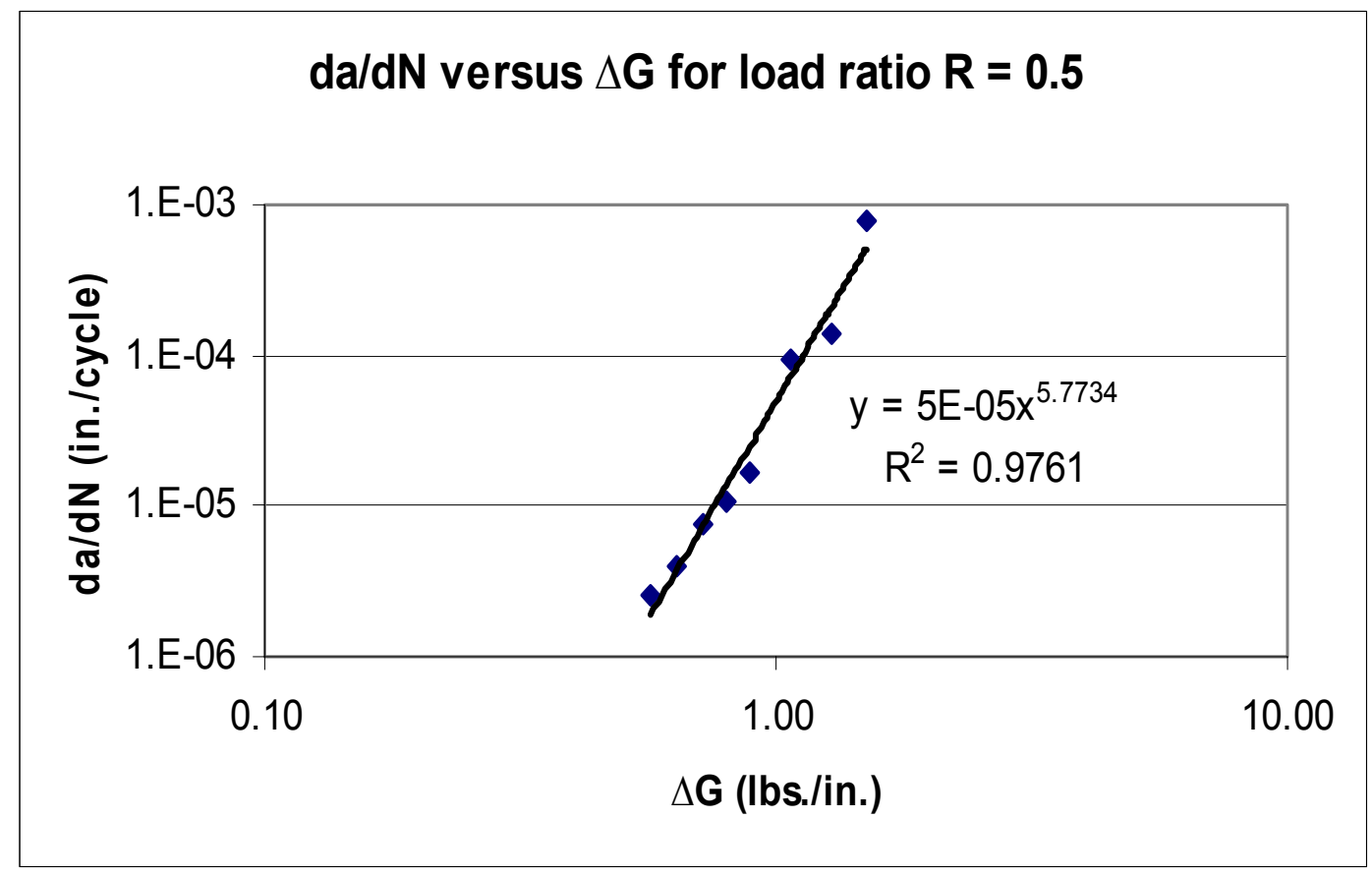

Fig. 3.29 The log-log plot of crack propagation rate $d a / d N$ versus the strain energy release rate range $\Delta G$ for load ratio $\mathrm{R}=0.5$ 


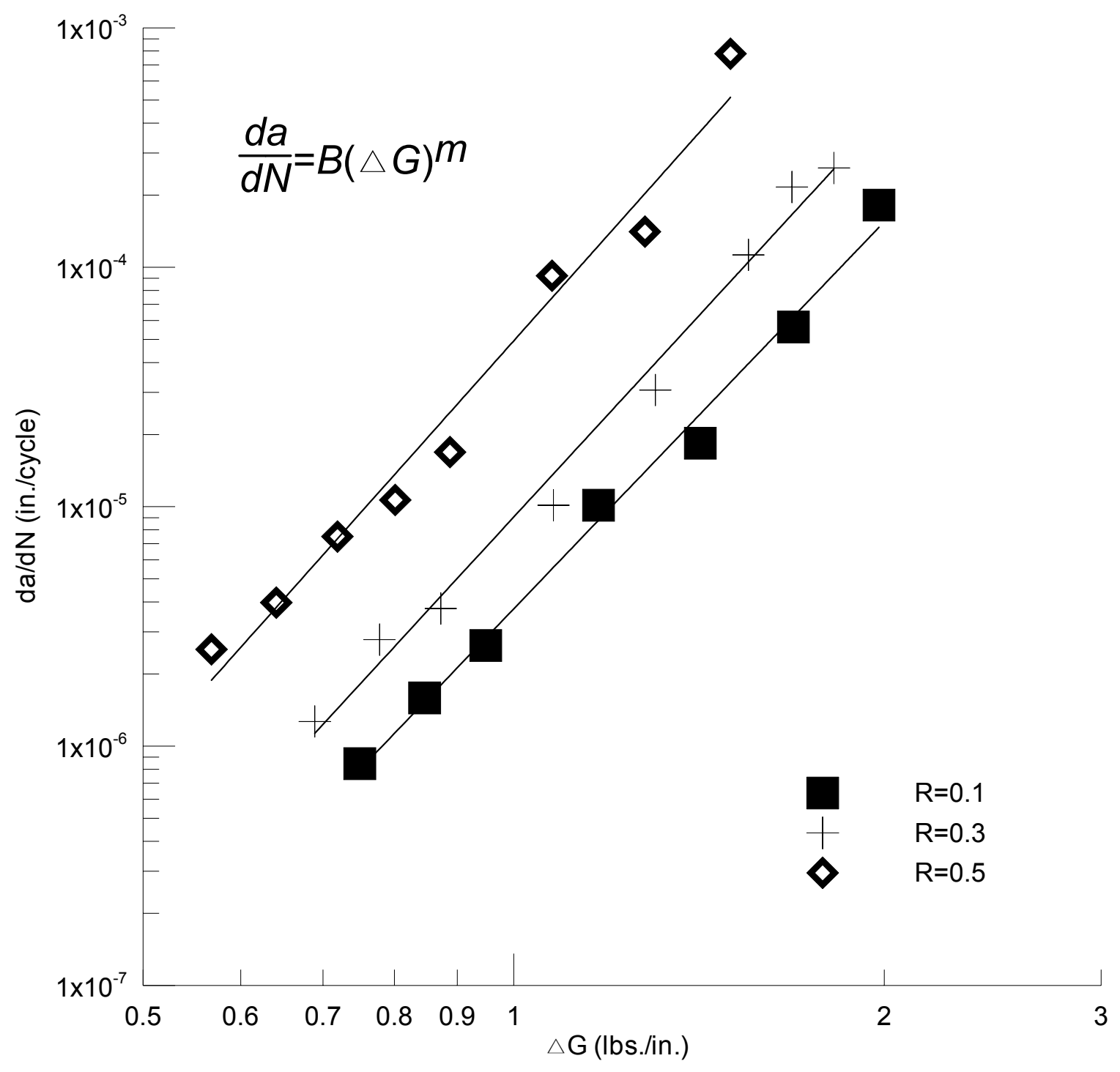

Fig. 3.30 The log-log plot of crack propagation rate $d a / d N$ versus the strain energy release rate range $\Delta G$ for different load ratios 
Arad et al. in 1971 proposed a modified Power Law equation including mean stress intensity factor effect given as

$$
\frac{d a}{d N}=B\left(K_{\max }^{2}-K_{\min }^{2}\right)^{m}=B\left(2 \Delta K K_{\text {mean }}\right)^{m}
$$

Applying the concept given in Eq. (3.25) to strain energy release rate, we can write the expression

$$
\frac{d a}{d N}=B\left(2 \Delta G G_{\text {mean }}\right)^{m}
$$

We use Eq. (3.26) to fit the experimental data and explain the load ratio effect. Fig. 3.31 gives the relationship of $d a / d N$ versus $2 \Delta G G_{\text {mean }}$. Even though the three fitting lines corresponding to the three different load ratios are closer to each other than in Fig. 3.30, the result is still not satisfactory.

Two interesting observations were found when examining the plots of $d a / d N$ vs. $G_{m i n}$ and $G_{m e a n}$. First, the relationship between the crack propagation rate $d a / d N$ and minimum strain energy release rate $G_{\min }$ is plotted in Fig. 3.32. Compared with Fig. 3.30, the effect of load ratio $R$ on the crack propagation rate $d a / d N$ in Fig. 3.32 is reversed; as the load ratio $R$ increases, the crack propagation rate $d a / d N$ decreases for the same minimum strain energy release rate $G_{\text {min }}$. Second, three lines corresponding to three different load ratios converge to a narrow band in the $\log -\log$ plot of $d a / d N$ versus $G_{\text {mean }}$ 
(see Fig. 3.33), and we conclude, therefore, that $G_{\text {mean }}$ is a very important parameter for load ratio effect and we explore its usefulness in this study.

As noticed by other researchers, strain energy release rate range $\Delta G$ is not the only controlling parameter for crack propagation rate $d a / d N$. Walker (1970) introduced a secondary controlling parameter and first defined the effective stress range as

$$
\overline{\Delta S}=S_{\max }^{1-\lambda} \Delta S^{\lambda}=S_{\max }(1-R)^{\lambda}
$$

The material constant $\lambda$ was given by Walker as approximately 0.5 for 2024-T3 aluminum and as 0.425 for 7075-T5 aluminum. Hojo et al. (1987) followed a similar procedure and defined equivalent stress intensity range as given in Eqs. (3.9) and (3.10). By applying Eq. (3.27) to strain energy release rate and using equivalent or effective strain energy release rate range concept, Eq. (3.27) can take the following form

$$
\Delta G_{e q}=G_{\max }(1-R)^{\lambda}
$$

Since $G_{\max }$ is kept constant for a given load ratio, we can write $G_{\max }=\frac{\Delta G}{1-R}$ to introduce $\Delta G$ in Eq. (3.28) as

$$
\Delta G_{e q}=\frac{\Delta G}{(1-R)^{1-\lambda}}
$$


Since minimum strain energy release rate $G_{\min }$ and strain energy release rate range $\Delta G$ have contrary effects on crack propagation rate $d a / d N$ for different load ratios $R$, we include minimum strain energy release rate $G_{\min }$ as a secondary controlling parameter to explain load ratio effect. Adopting Walker's idea on effective stress range and following a similar approach as Hojo et al. (1987), we first define the equivalent strain energy release rate range as

$$
\Delta G_{e q}=\Delta G^{(1-\lambda)} G_{\min }^{\lambda}
$$

Sutton (1974) pointed out that any two of the following parameters: $G_{\min }, G_{\max }, \Delta G$, $G_{\text {mean }}$, and $R$ can characterize crack growth rate under different load ratios. We already noticed from Fig. 3.33 that $G_{m e a n}$ is a very important parameter for load ratio effect, and therefore, we modify Eq. (3.30) to reflect the effect of $G_{\text {mean }}$ in $\Delta G_{e q}$

$$
\Delta G_{e q}=\Delta G^{(1-\lambda)} G_{\text {mean }}^{\lambda}
$$

The effect of load ratio $R$ in $\Delta G_{e q}$ as a secondary controlling parameter is reflected in Eq. (3.29). Then the modified Paris Law equation is expressed as

$$
\frac{d a}{d N}=B\left(\Delta G_{e q}\right)^{m}
$$


In Eqs. (3.29), (3.30) and (3.31), $\lambda$ is a material constant, which defines the relative importance of contribution of $R, G_{\min }$ and $G_{\text {mean }}$, respectively, to the equivalent or effective strain energy release rate range $\Delta G_{e q}$ on crack growth rate. The constant $\lambda$ is obtained by trial and error to best fit the data points. The final log-log plots of experimental data fitted by the modified Paris Law equation (see Eqs. 3.29, 3.30, 3.31 and 3.32) are shown in Figs. 3.34, 3.35 and 3.36, and the best least squares fit results for material constants $B, m$ and $\lambda$ and coefficients of determination $r^{2}$ are given in Table 3.12.

Table 3.12 Material constants $B, m$ and $\lambda$ and coefficient of determination $r^{2}$ for three modified Paris Law equations

\begin{tabular}{|c|c|c|c|c|}
\hline $\begin{array}{c}\text { Modified Paris Law } \\
\text { equation }\end{array}$ & $B$ & $m$ & $\lambda$ & $r^{2}$ \\
\hline$\frac{d a}{d N}=B\left(\frac{\Delta G}{(1-R)^{1-\lambda}}\right)^{m}$ & $2 \times 10^{-6}$ & 5.54 & 0.21 & 0.98 \\
\hline$\frac{d a}{d N}=B\left(\Delta G^{(1-\lambda)} G_{\min }^{\lambda}\right)^{m}$ & $7 \times 10^{-5}$ & 5.34 & 0.13 & 0.95 \\
\hline$\frac{d a}{d N}=B\left(\Delta G^{(1-\lambda)} G_{\text {mean }}^{\lambda}\right)^{m}$ & $1 \times 10^{-4}$ & 5.59 & 0.93 & 0.99 \\
\hline
\end{tabular}

Where $r^{2}$ (Mendenhall 1999) is coefficient of determination and $1 \geq r^{2} \geq 0$. If there is no random variation and all points fall on the regression line, $r^{2}=1$. If the points are randomly scattered and there is no variation explained by regression, $r^{2}=0$. 


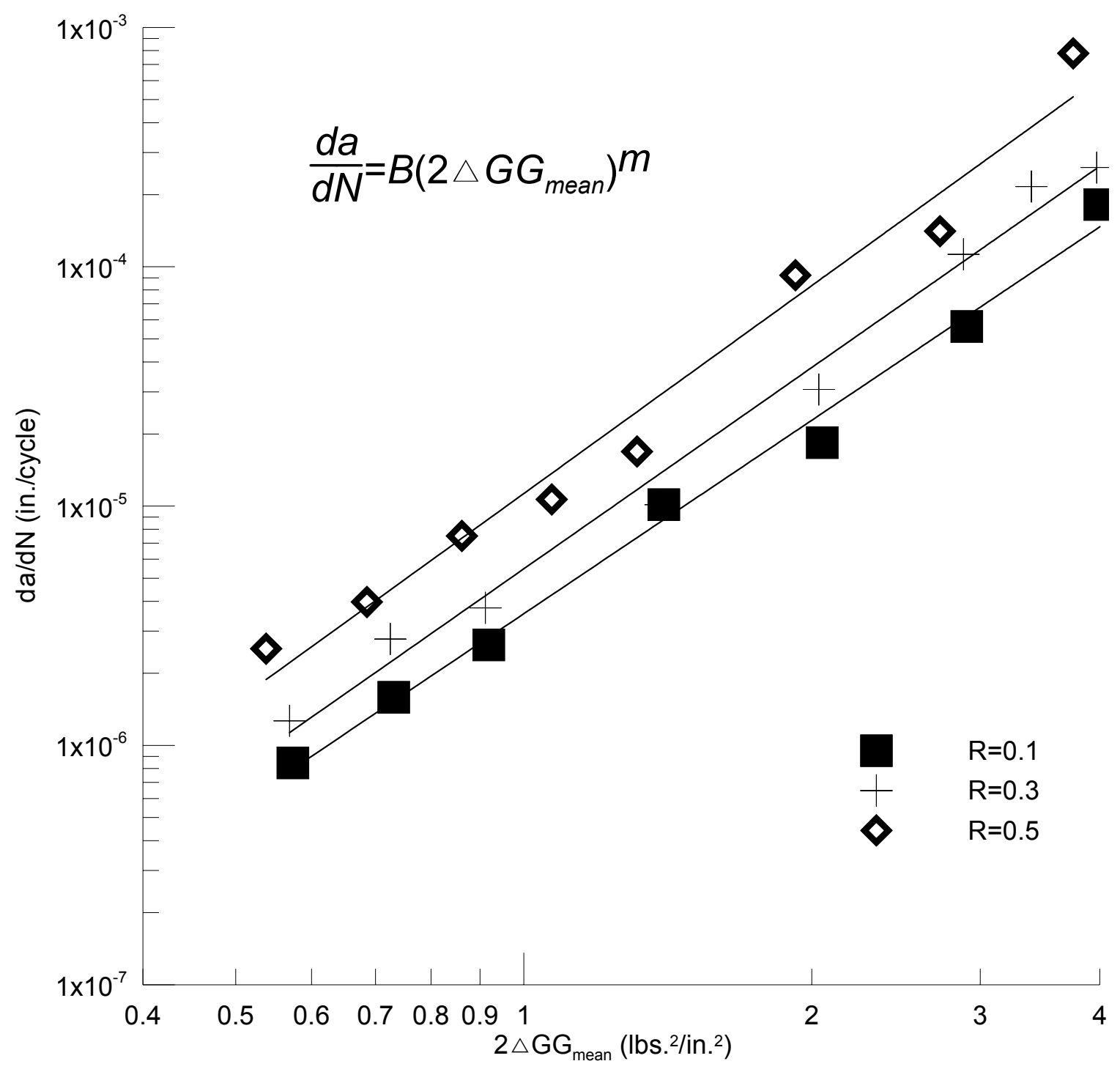

Fig. 3.31 The log-log plot of crack propagation rate $d a / d N$ versus the strain energy release rate range $2 \Delta G G_{\text {mean }}$ for different load ratios 


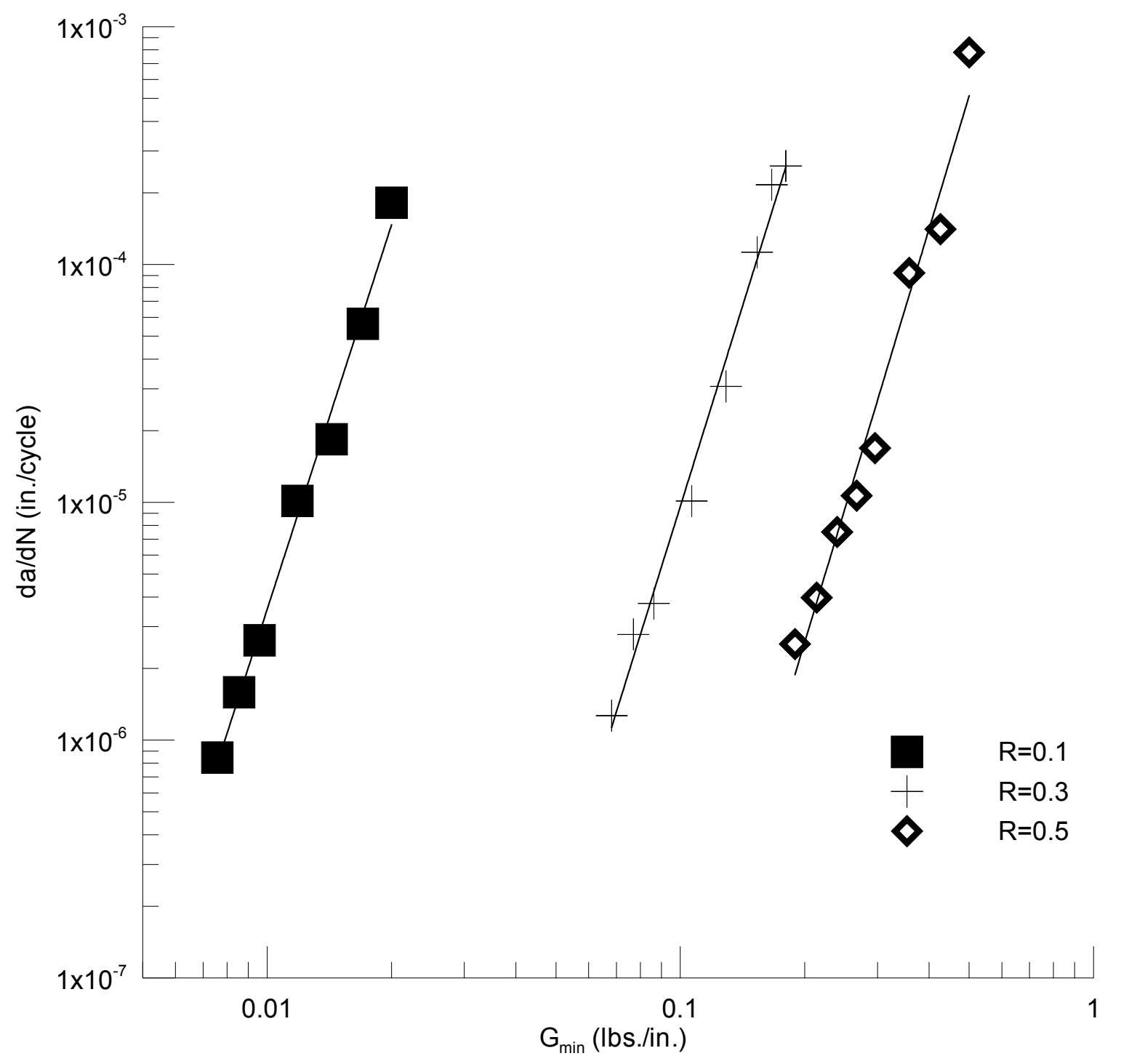

Fig. 3.32 The log-log plot of crack propagation rate $d a / d N$ versus the strain energy release rate range $G_{\min }$ for different load ratios 


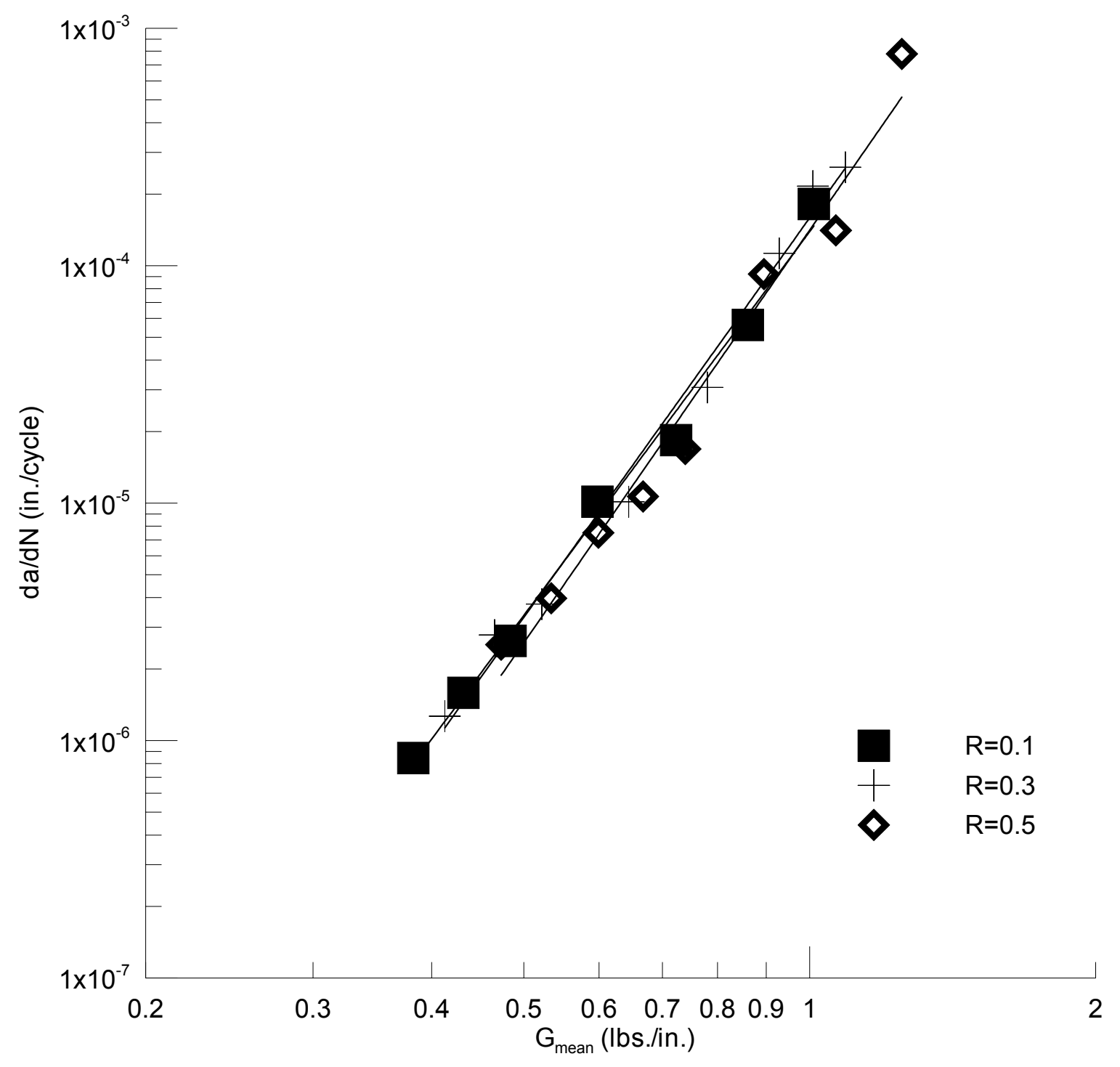

Fig. 3.33 The log-log plot of crack propagation rate $d a / d N$ versus the strain energy release rate range $G_{\text {mean }}$ for different load ratios 


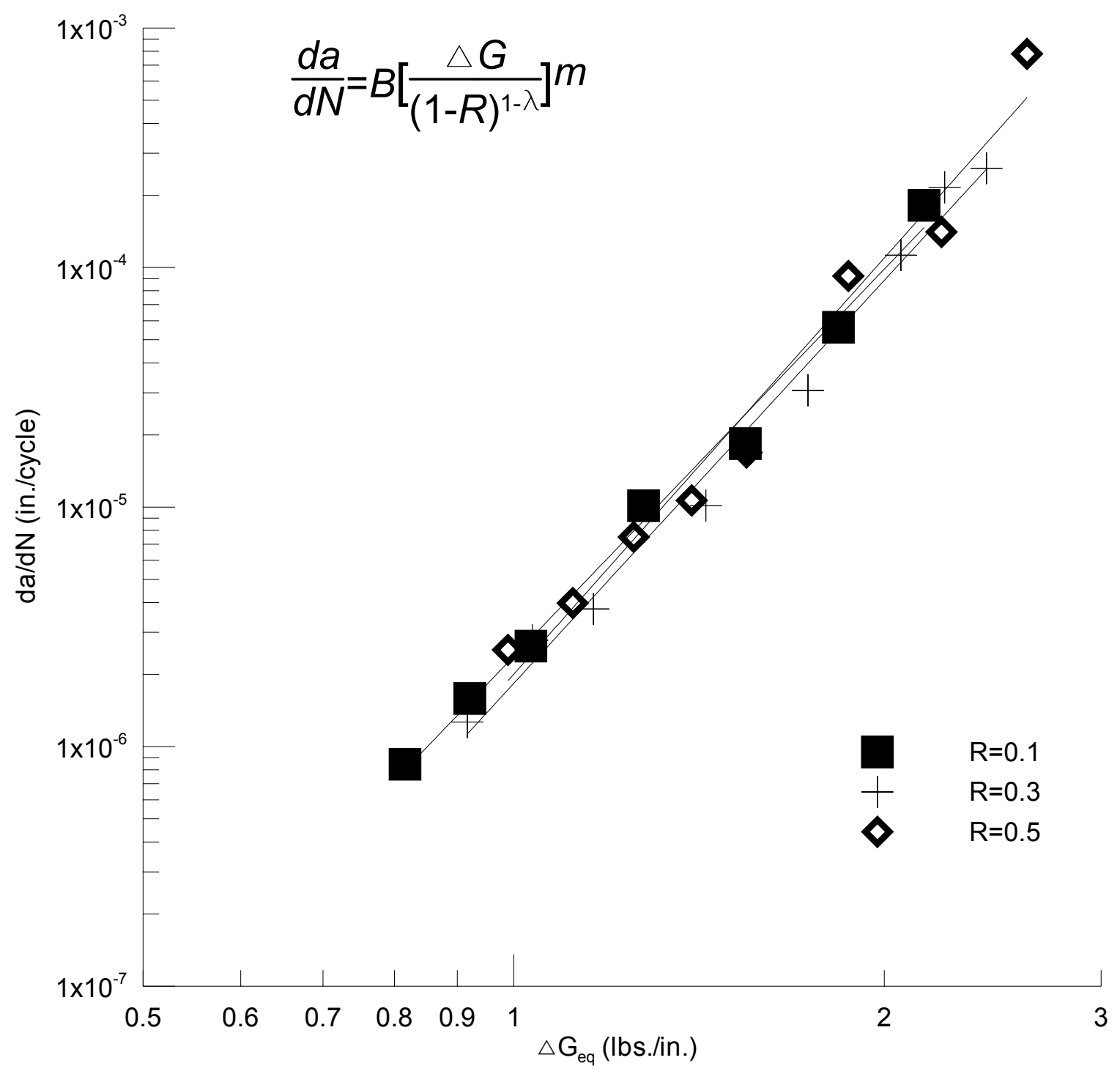

Fig. 3.34 The log-log plot of crack propagation rate $d a / d N$ versus the strain energy release rate range $\Delta G /(1-R)^{1-\lambda}$ for different load ratios 


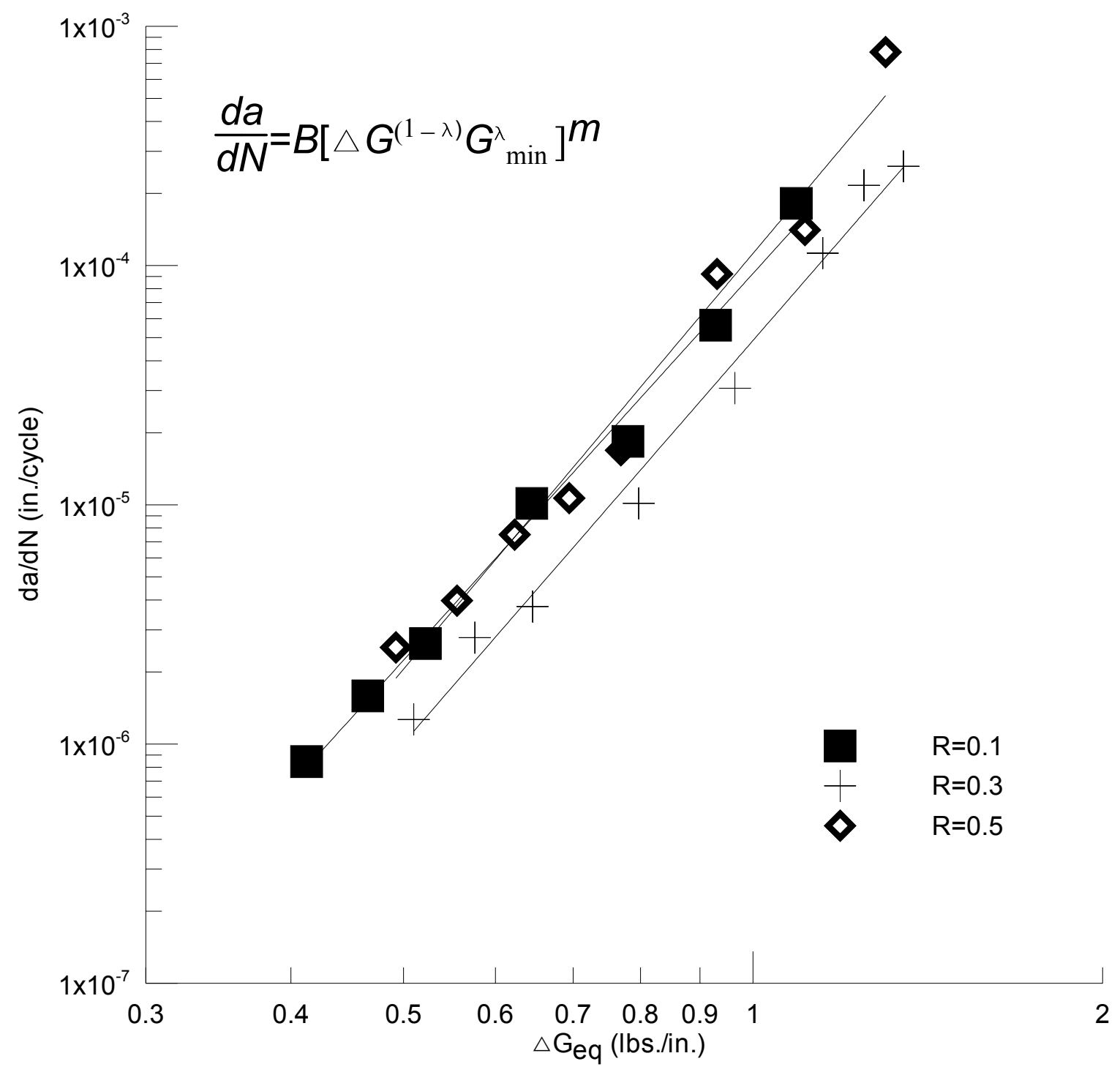

Fig. 3.35 The log-log plot of crack propagation rate $d a / d N$ versus the strain energy release rate range $\Delta G^{(1-\lambda)} G^{\lambda}$ min for different load ratios 


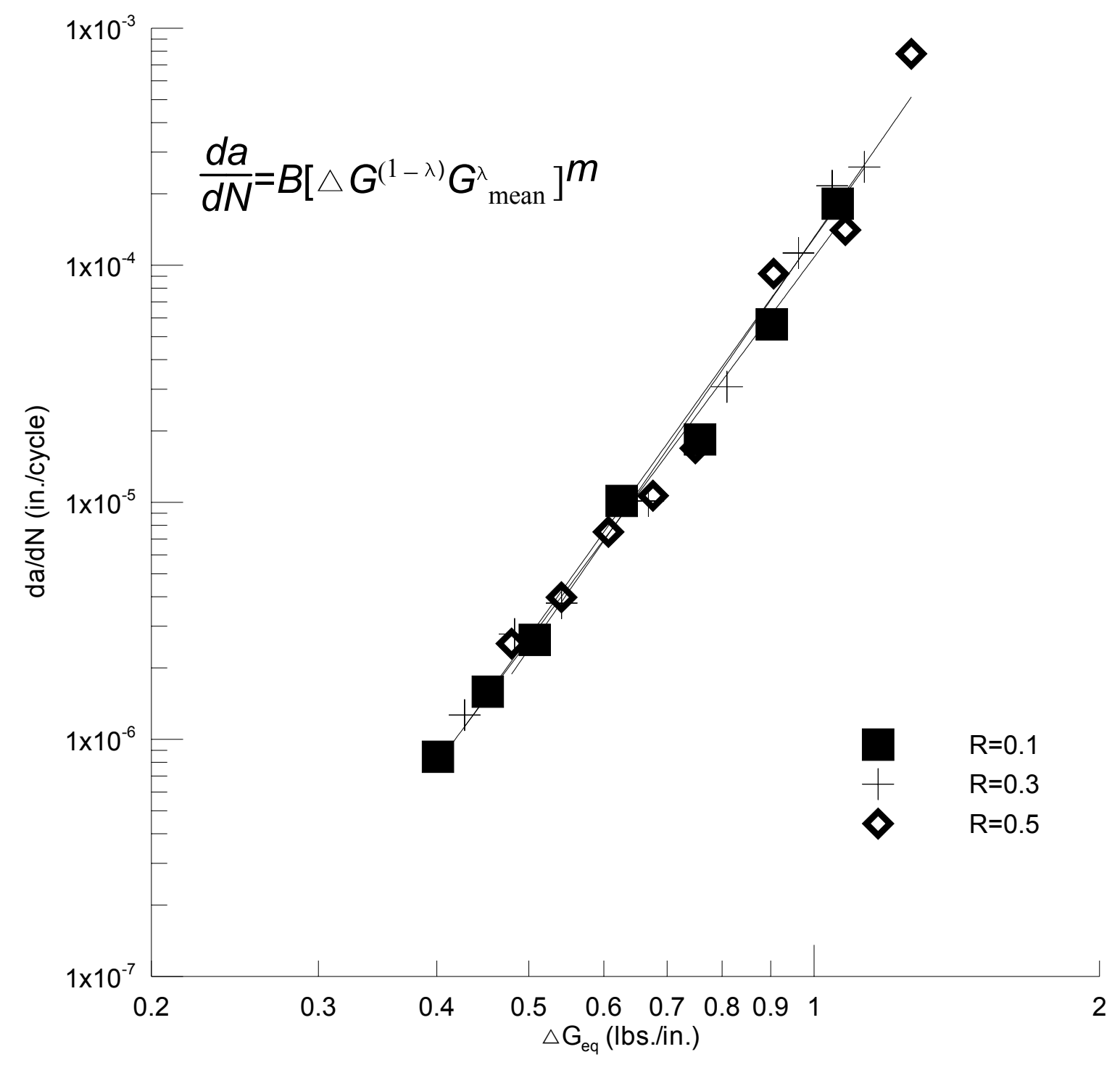

Fig. 3.36 The log-log plot of crack propagation rate $d a / d N$ versus the strain energy release rate range $\Delta G^{(1-\lambda)} G_{\text {mean }}^{\lambda}$ for different load ratios 
The equivalent strain energy release rate range for three kinds of combinations of $\Delta G$ and $R, \Delta G$ and $G_{\min }$ and $\Delta G$ and $G_{\text {mean }}$ all give us strong linear correlations ( $r$ is nearly equal to 1). This confirms Sutton's (1974) assertion that any two of the following parameters: $G_{\min }, G_{\max }, \Delta G, G_{\text {mean }}$, and $R$ can characterize crack growth rate under different load ratios. Of the present three relations, $\Delta G$ and $G_{\text {mean }}$ combination is the best among the curve fitting plots since the coefficient of determination is closest to 1 . Therefore, we strongly recommend that the modified Power Law equation for the combination of $\Delta G$ and $G_{m e a n}$ be used in engineering practice.

\subsubsection{The crack closure effect}

Usually the crack closure has been considered only to occur at compressive loads. Under cyclic tension loading, crack closure has been excluded in the stress analysis of the crack tip, but this assumption can only apply to an idealized crack that is not propagating. Partial or complete crack closure is expected during the unloading period. Elber (1970 and 1971) first rationalized the possibility of fatigue crack closure even at a far-field tensile load based on experimental observations. The results of Elber have shown that a fatigue crack propagating under zero-to-tension loading was fully closed at zero loads due to internal forces.

Due to the crack closure effect, the local crack driving force is reduced from the applied stress intensity factor range, $\Delta K$ to an effective stress intensity range, $\Delta K_{\text {eff. }}$ The effective stress intensity range, $\Delta K_{\text {eff }}$ is defined as 


$$
\Delta K_{e f f}=K_{\max }-K_{o p}=\alpha \cdot \Delta K
$$

where $K_{o p}$ is the stress intensity factor at which the crack opens fully, and $\alpha$ is an effective stress intensity range ratio and is defined as

$$
\alpha=\frac{K_{\max }-K_{o p}}{K_{\max }-K_{\min }}=\frac{\Delta K_{e f f}}{\Delta K}
$$

Elber argued that this is very important to characterize the kinetics of fatigue crack growth since only the period when crack is open contributes to crack propagation. The corresponding characterization of the fatigue crack propagation rate based on the effective stress intensity range, $\Delta K_{\text {eff }}$ becomes

$$
\frac{d a}{d N}=B\left(\Delta K_{e f f}\right)^{m}=B(\alpha \Delta K)^{m}
$$

In the present study, the crack closure effect is also observed for phenolic FRPred maple wood bonded interface under tension-tension cyclic loading, especially for load ratio of 0.1 . Coincidentally, the plot of crack growth rate $d a / d N$ versus $G_{\text {mean }}$ seems to give a good fit of the data (see Fig. 3.33). One is led to the conclusion that in these tests the equivalent or effective strain energy release rate range $\Delta G_{e q}$ is approximately equal to the average strain energy release rate $G_{\text {mean }}$. 


$$
\Delta G_{e q}=G_{\text {mean }}
$$

Similar to an effective or equivalent stress intensity range ratio (see Eq. 3.35), let us define an effective or equivalent strain energy release rate range ratio as

$$
\alpha=\frac{G_{\max }-G_{o p}}{G_{\max }-G_{\min }}=\frac{\Delta G_{e f f}}{\Delta G}
$$

From Eq. (3.36), a relationship on effective or equivalent strain energy release rate range ratio is derived

$$
\Delta G_{\text {eq }}=\left(G_{\text {mean }} \frac{1}{\Delta G}\right) \Delta G=\alpha \cdot \Delta G
$$

Then, the relationship between effective or equivalent strain energy release rate range ratio $\alpha$ and load ratio $R$ based on Eq. (3.38) can be expressed as

$$
\alpha=\frac{G_{\max }+G_{\min }}{2} \times \frac{1}{\Delta G}=\frac{G_{\min }}{\Delta G}+0.5=\frac{R}{1-R}+0.5
$$

The results of Elber's work is given as

$$
\alpha=0.4 R+0.5
$$


where $-0.1<R<0.7$ for 2024-T3 aluminum alloy. Surprisingly, the relationships between effective or equivalent strain energy release rate range ratio $\alpha$ and load ratio $R$ in Eqs. (3.39) and (3.40) have some similarity, even though Eq. (3.40) gives a linear relationship. For comparison of the effective or equivalent strain energy release rate range ratio $\alpha$ between Eqs. (3.39) and (3.40), $\alpha$ has been computed for load ratios $R$ of 0.0 to 0.7 . These are listed in Table 3.13 .

Table 3.13 Comparison of the effective or equivalent strain energy release rate range ratio $\alpha$ in Eqs. (3.39) and (3.40)

\begin{tabular}{ccc}
\hline Load ratio R & $\alpha$ in Eq. (3.40) & $\alpha$ in Eq. (3.39) \\
\hline 0.0 & 0.50 & 0.50 \\
0.1 & 0.54 & 0.61 \\
0.2 & 0.58 & 0.75 \\
0.3 & 0.62 & 0.93 \\
0.4 & 0.66 & 1.17 \\
0.5 & 0.70 & 1.50 \\
0.6 & 0.74 & NA \\
0.7 & 0.78 & NA \\
\hline
\end{tabular}

From Table 3.13, one can find that the effective or equivalent strain energy release rate range ratio $\alpha$ in Eq. (3.40) increases with a very small step as load ratio $R$ increases, and it is smaller than one even at a load ratio $R$ as high as 0.7 . In our case, in contrast, at the 
highest $R=0.5$ considered in this study, $\alpha$ is larger than 1.0. Obviously there is a critical load ratio below which the minimum load is less than the crack opening load. Let us define the critical load ratio $R_{\text {critical }}$ as the load ratio at which the effective or equivalent strain energy release rate range ratio $\alpha$ equal to 1 , that is crack opening load equal to minimum cyclic load. The critical load ratio $R_{\text {critical }}$ is computed as 0.33 from setting Eq. (3.39) equal to 1 . On the other hand, the critical load ratio $R_{\text {critical }}$ can not be computed from Eq. (3.40) and it must be greater than 0.7. The approximate value of critical load ratio $R_{\text {critical }}$ defined here can be further explored in a future study.

Eq. (3.39) suggests that only a fraction of $\Delta G$ during the load cycle caused the crack to fully open, particularly for $R=0.1$. It is, therefore, reasonable to relate the crack growth rate $d a / d N$ to $\alpha \Delta G$, as

$$
\frac{d a}{d N}=f(\alpha \cdot \Delta G)
$$

where $\alpha$ is an effective or equivalent energy release rate range ratio (see Eq. 3.37). The conclusions in section 3.4.4 on a modified Paris Law equation then can be extended to $\Delta G$ combine with any of the following parameters: $R, G_{\text {min }}, G_{m e a n}$, and $\alpha$ to explain the load ratio effect for the phenolic FRP-red maple wood bonded interface. 


\subsubsection{The failure surface}

The photographs of the fracture surface for the phenolic FRP-red maple wood CDCB specimen are shown in Figs. 3.37 through 3.59 for the three groups of load ratios tested. Observations of the fracture surface show that the interface adhesive failure is predominantly the failure mode of the specimens. It is also found that some of the fractured surfaces happened within the continuous strand mat (CSM) layer of the FRP substrate in combination with interface adhesive failure (see Figs. 3.37 and 3.39). For several specimens, substantial fiber bridging was evident during the fracture process. An example is shown in Fig. 3.60. The specimens showing substantial bridging effects were disregarded in order to concentrate on interface failure. Failures around the loading pins and within the contoured portions of CDCB specimens, which happened often in static tests, did not occur in the fatigue tests. The reason for this is probably that the fatigue loading is lower than the critical static loading. Figures of 3.37 through 3.59 are typical of the interface bond failure, which is the primary failure mode for the specimens in this study. 


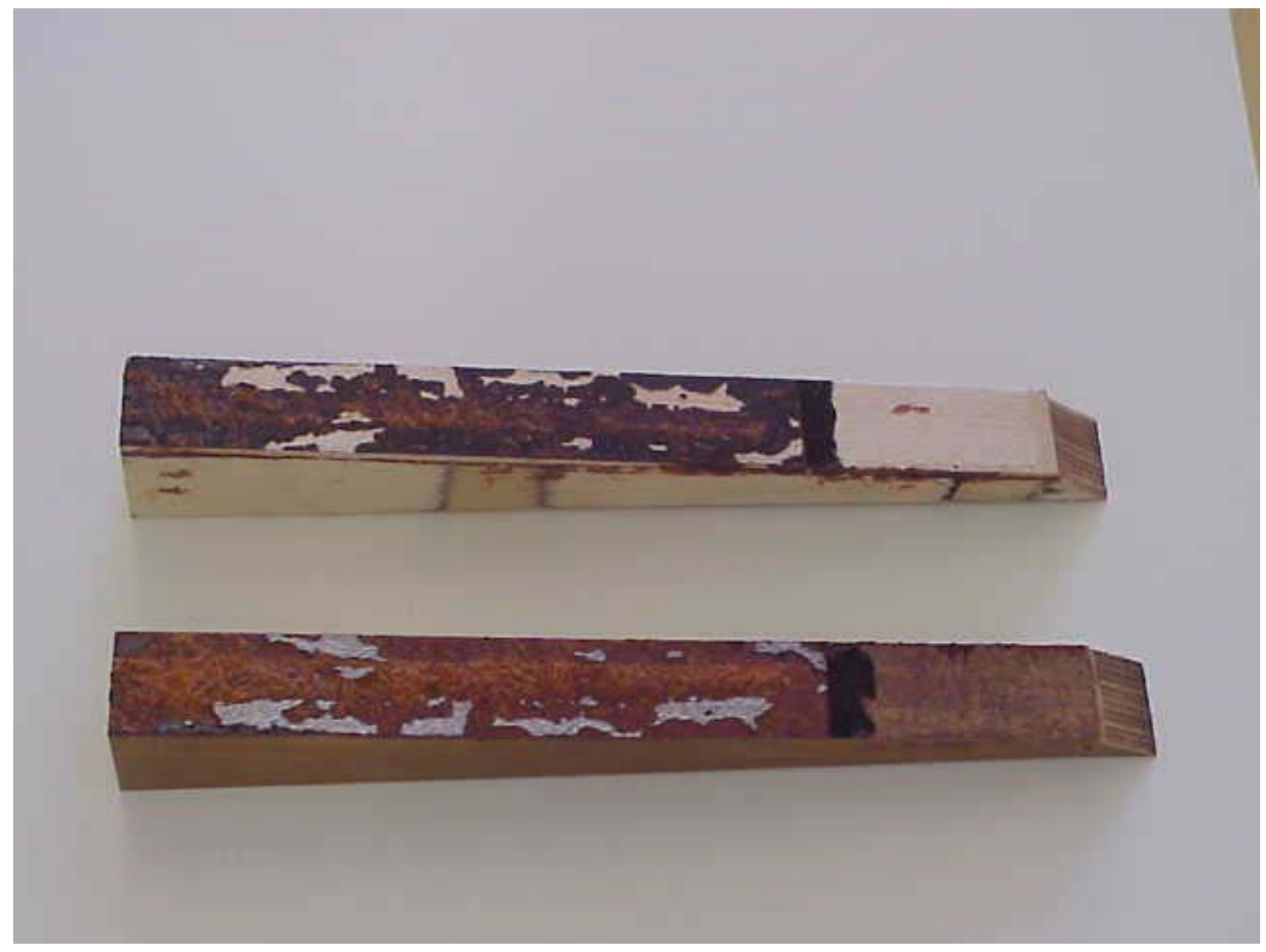

Fig. 3.37 Fractured faces for a CDCB specimen under 8-80 lb. cyclic loading with a frequency $f=1 \mathrm{~Hz}$, load ratio $R=0.1$ and sinusoidal waveform 


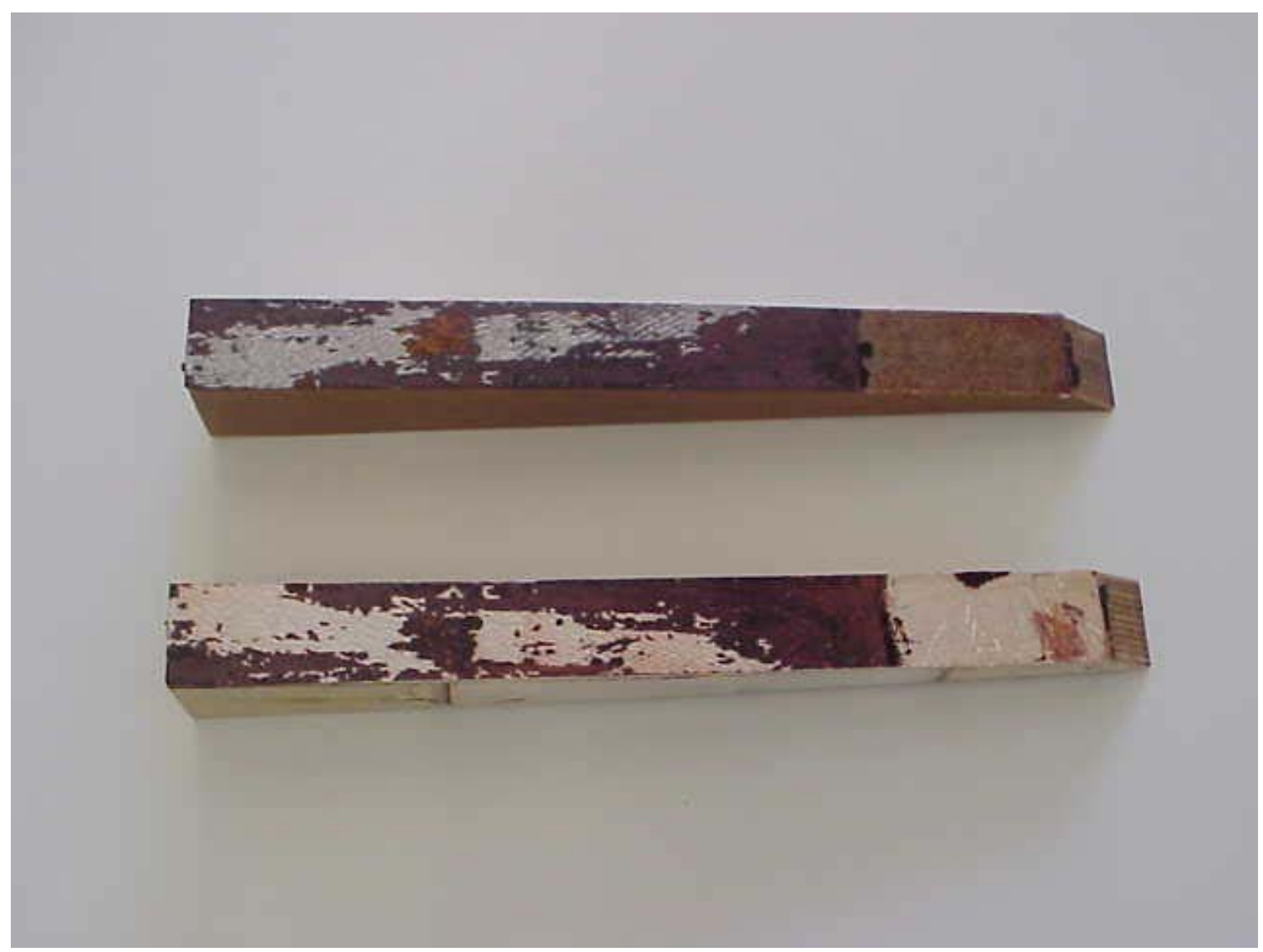

Fig. 3.38 Fractured faces for a CDCB specimen under 8.5-85 lb. cyclic loading with a frequency $f=1 \mathrm{~Hz}$, load ratio $R=0.1$ and sinusoidal waveform 


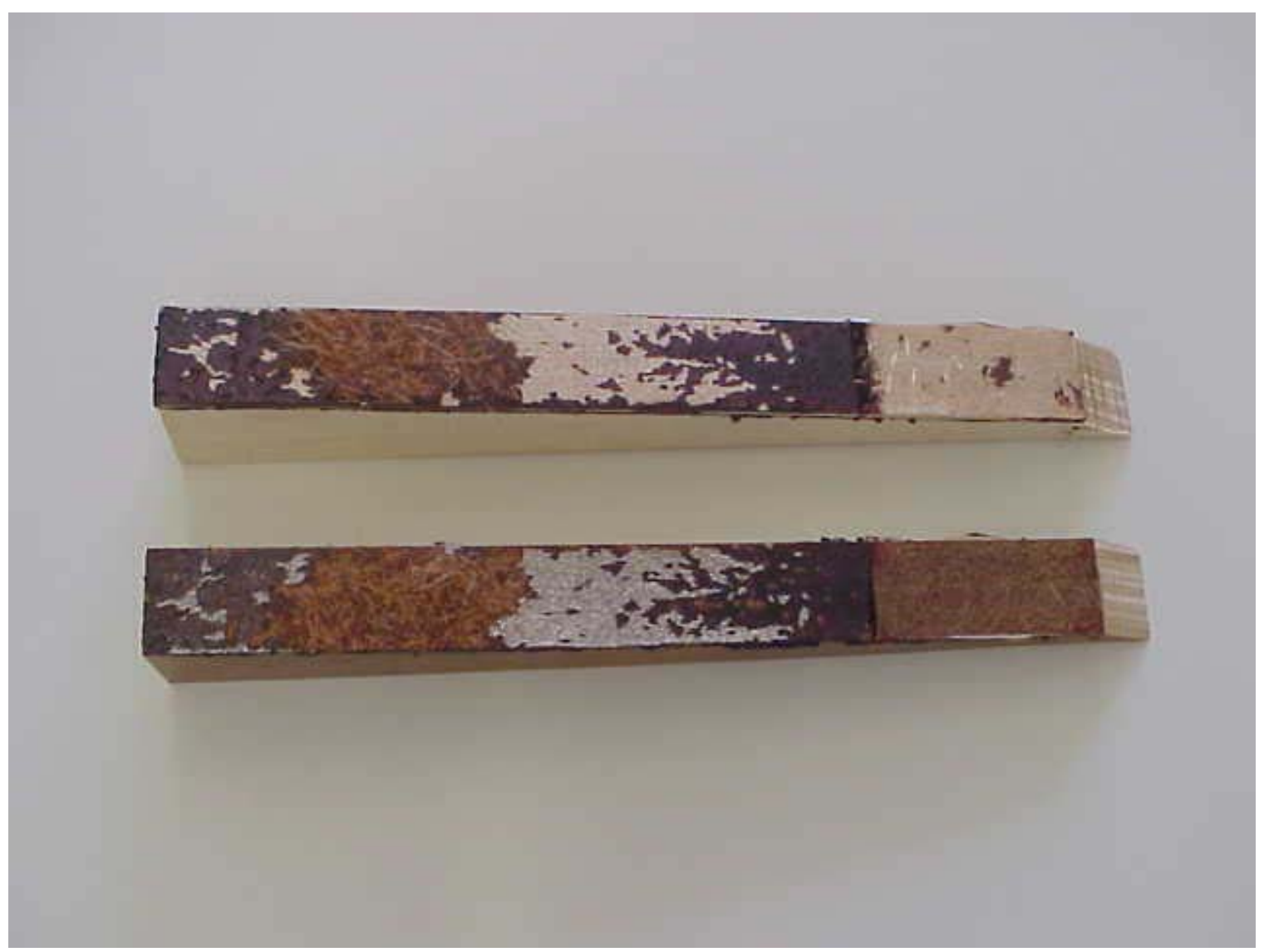

Fig. 3.39 Fractured faces for a CDCB specimen under 9-90 lb. cyclic loading with a frequency $f=1 \mathrm{~Hz}$, load ratio $R=0.1$ and sinusoidal waveform 


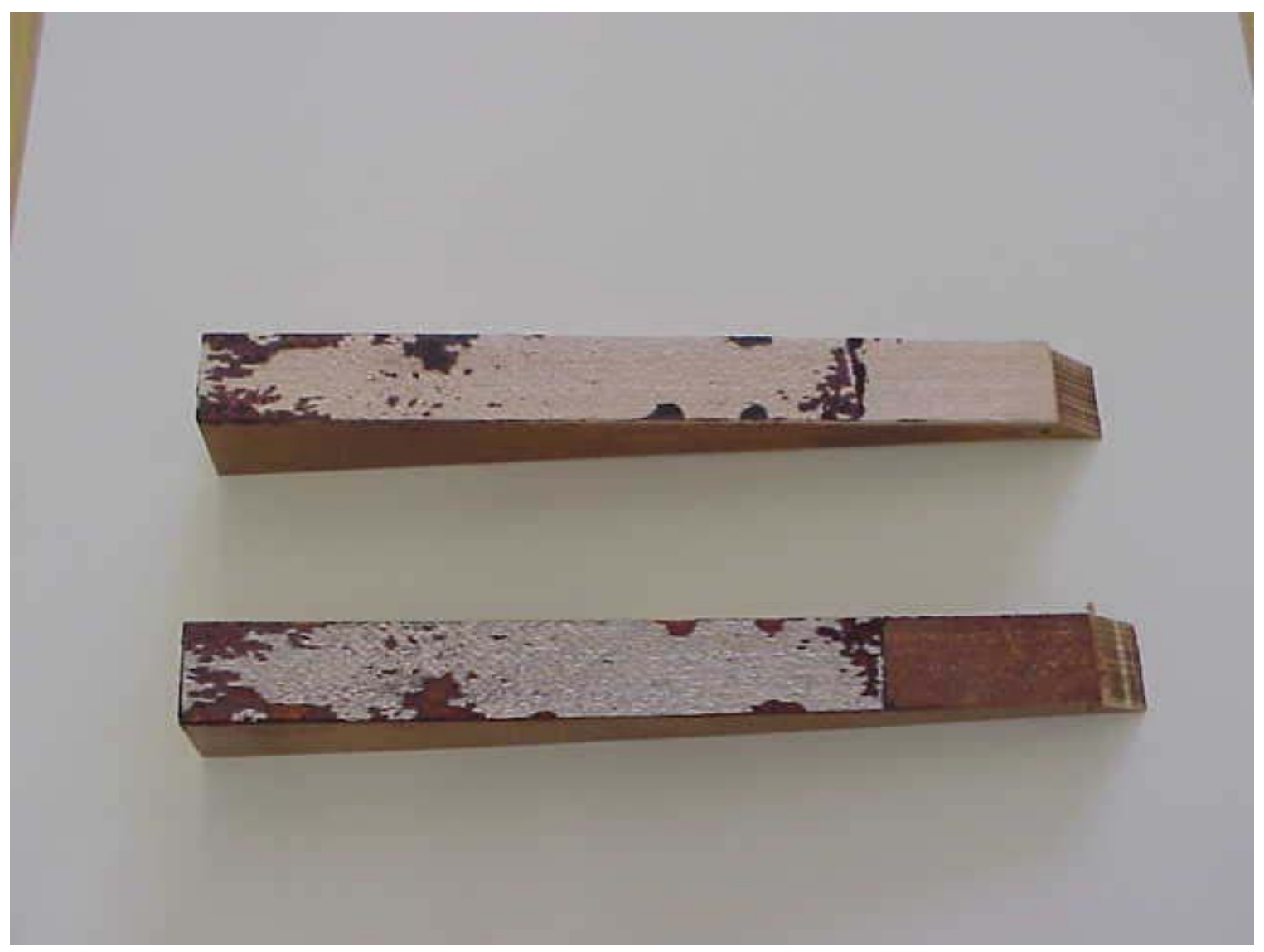

Fig. 3.40 Fractured faces for a CDCB specimen under 10-100 lb. cyclic loading with a frequency $f=1 \mathrm{~Hz}$, load ratio $R=0.1$ and sinusoidal waveform 


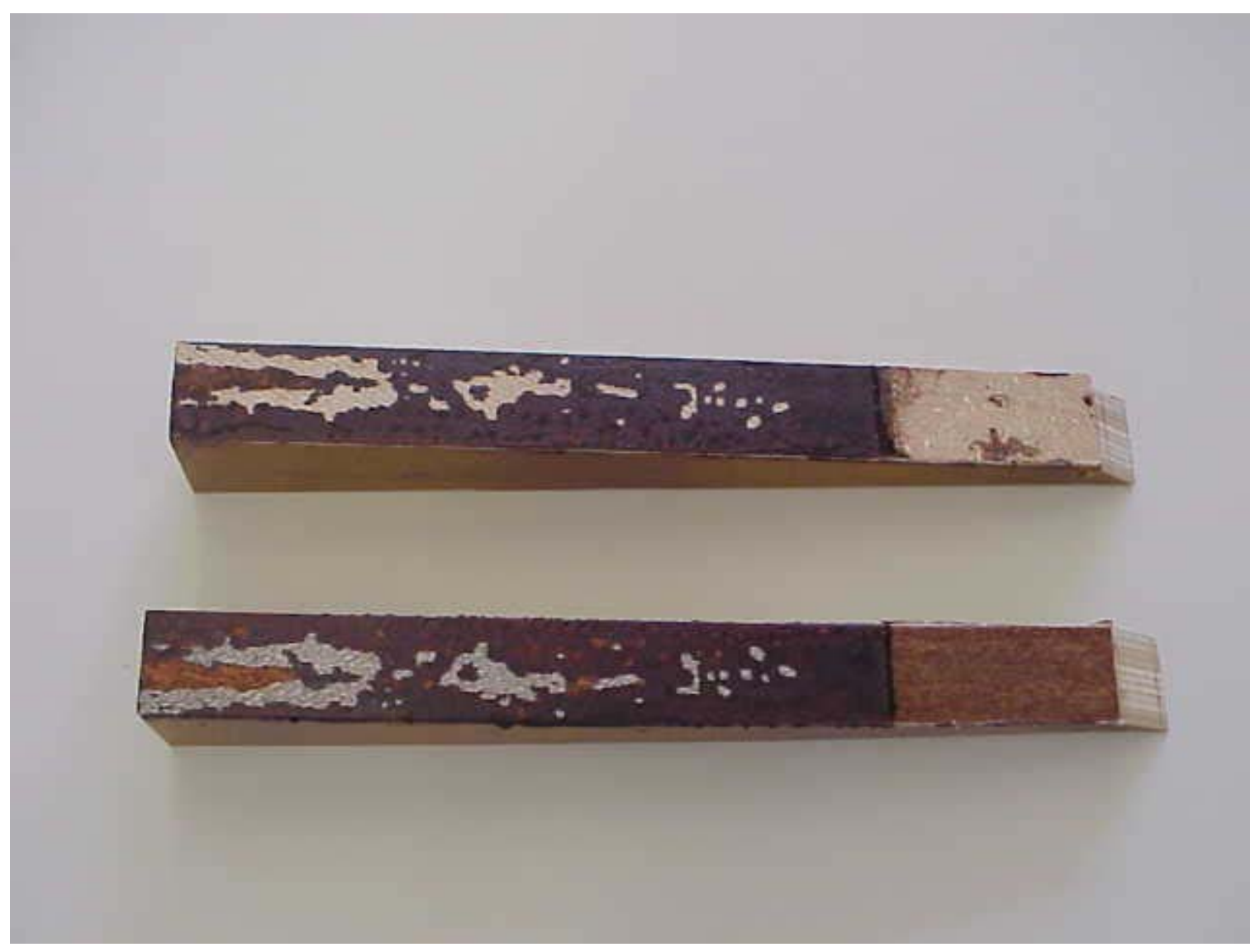

Fig. 3.41 Fractured faces for a CDCB specimen under 11-110 lb. cyclic loading with a frequency $f=1 \mathrm{~Hz}$, load ratio $R=0.1$ and sinusoidal waveform 


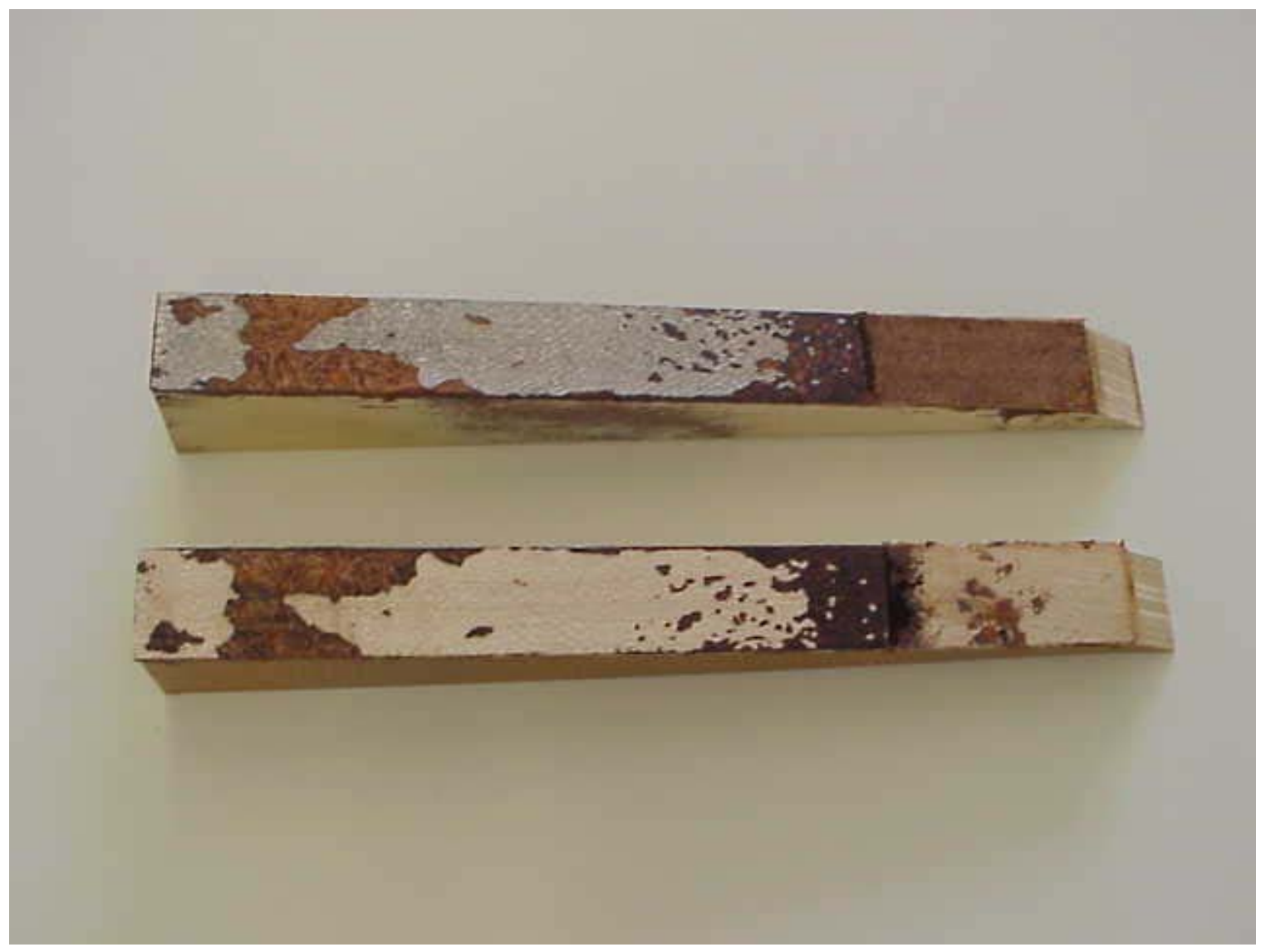

Fig. 3.42 Fractured faces for a CDCB specimen under 12-120 lb. cyclic loading with a frequency $f=1 \mathrm{~Hz}$, load ratio $R=0.1$ and sinusoidal waveform 


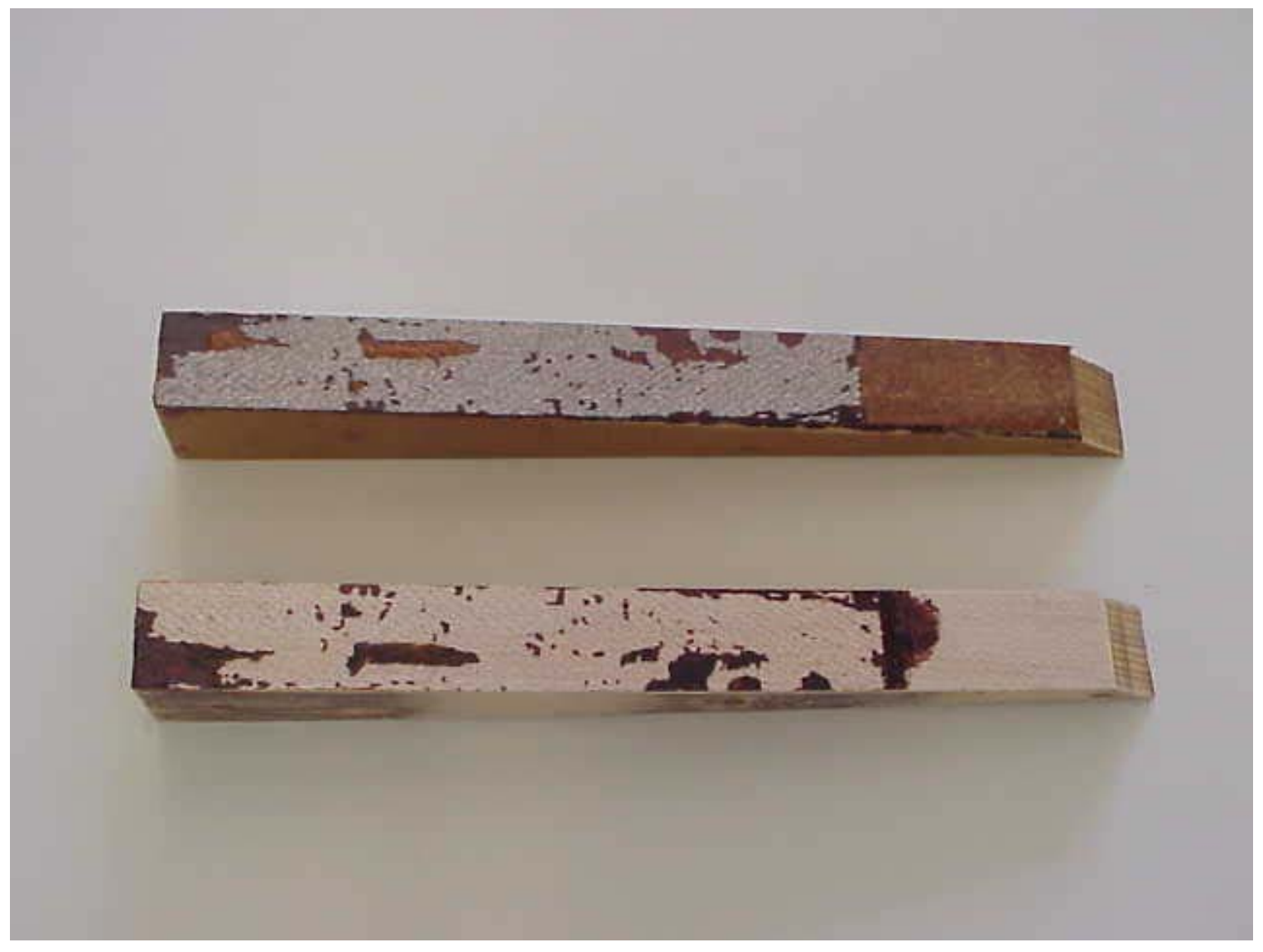

Fig. 3.43 Fractured faces for a CDCB specimen under 13-130 lb. cyclic loading with a frequency $f=1 \mathrm{~Hz}$, load ratio $R=0.1$ and sinusoidal waveform 


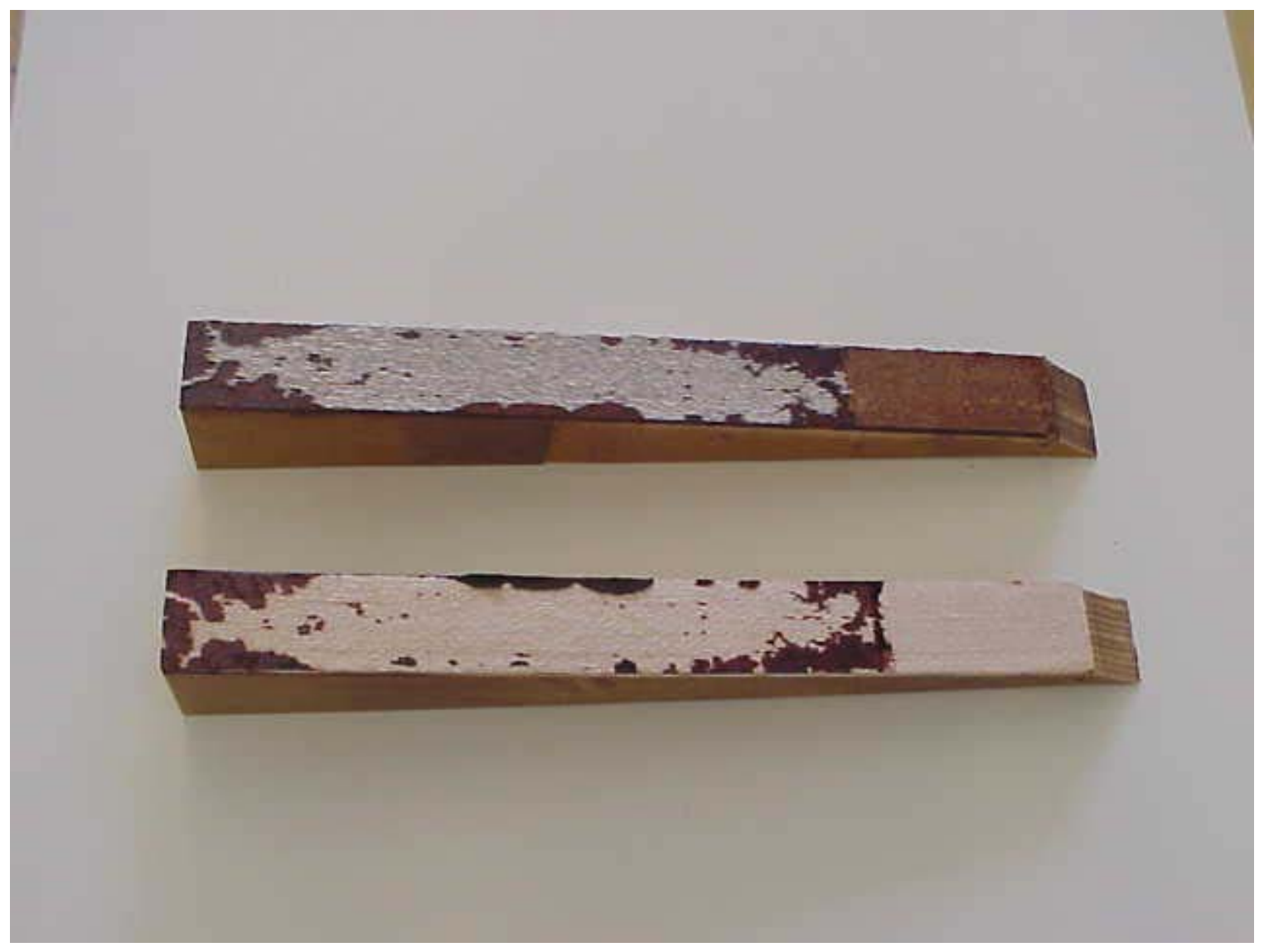

Fig. 3.44 Fractured faces for a CDCB specimen under 24-80 lb. cyclic loading with a frequency $f=1 \mathrm{~Hz}$, load ratio $R=0.3$ and sinusoidal waveform 


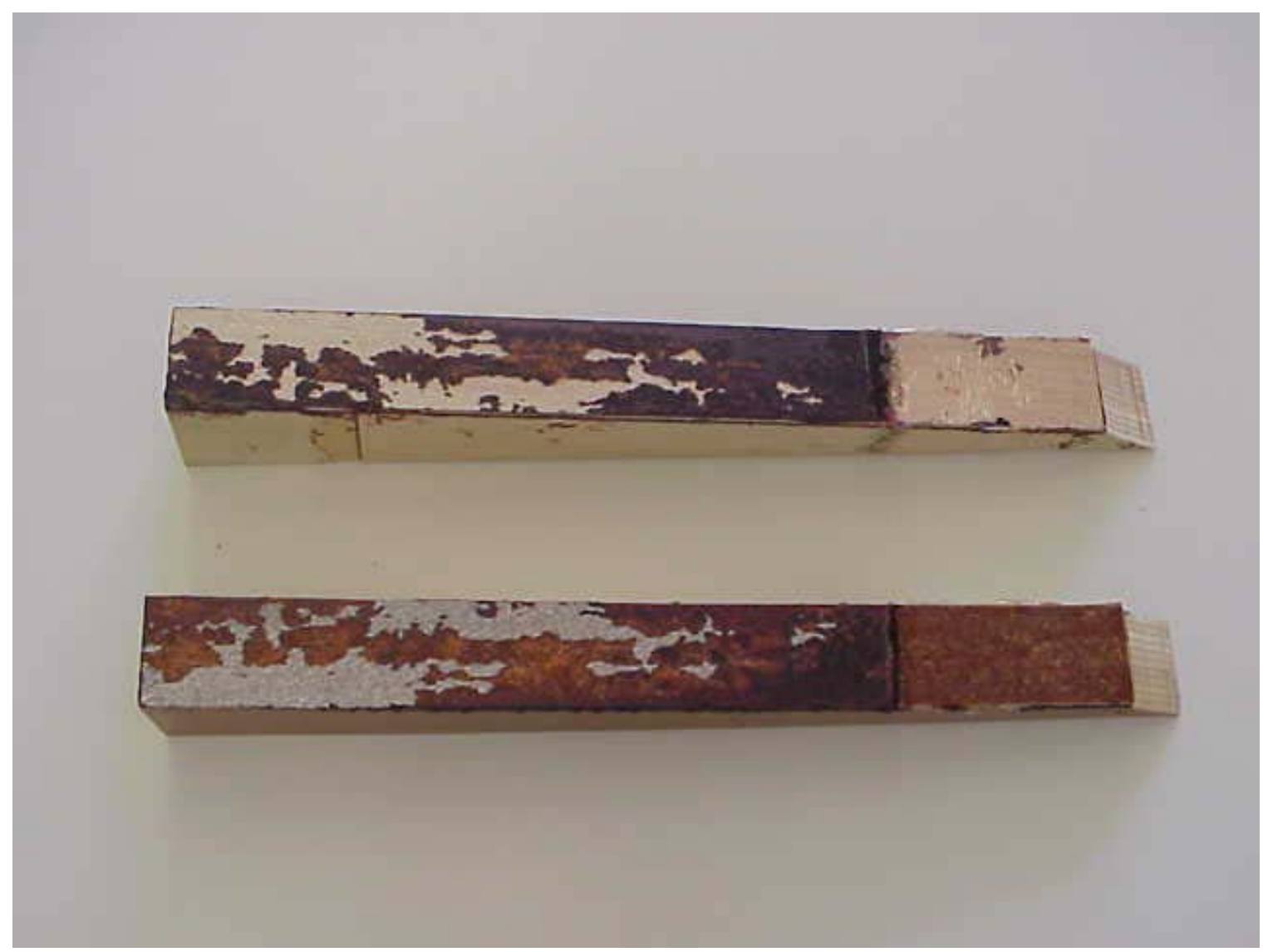

Fig. 3.45 Fractured faces for a CDCB specimen under 25.5-85 lb. cyclic loading with a frequency $f=1 \mathrm{~Hz}$, load ratio $R=0.3$ and sinusoidal waveform 


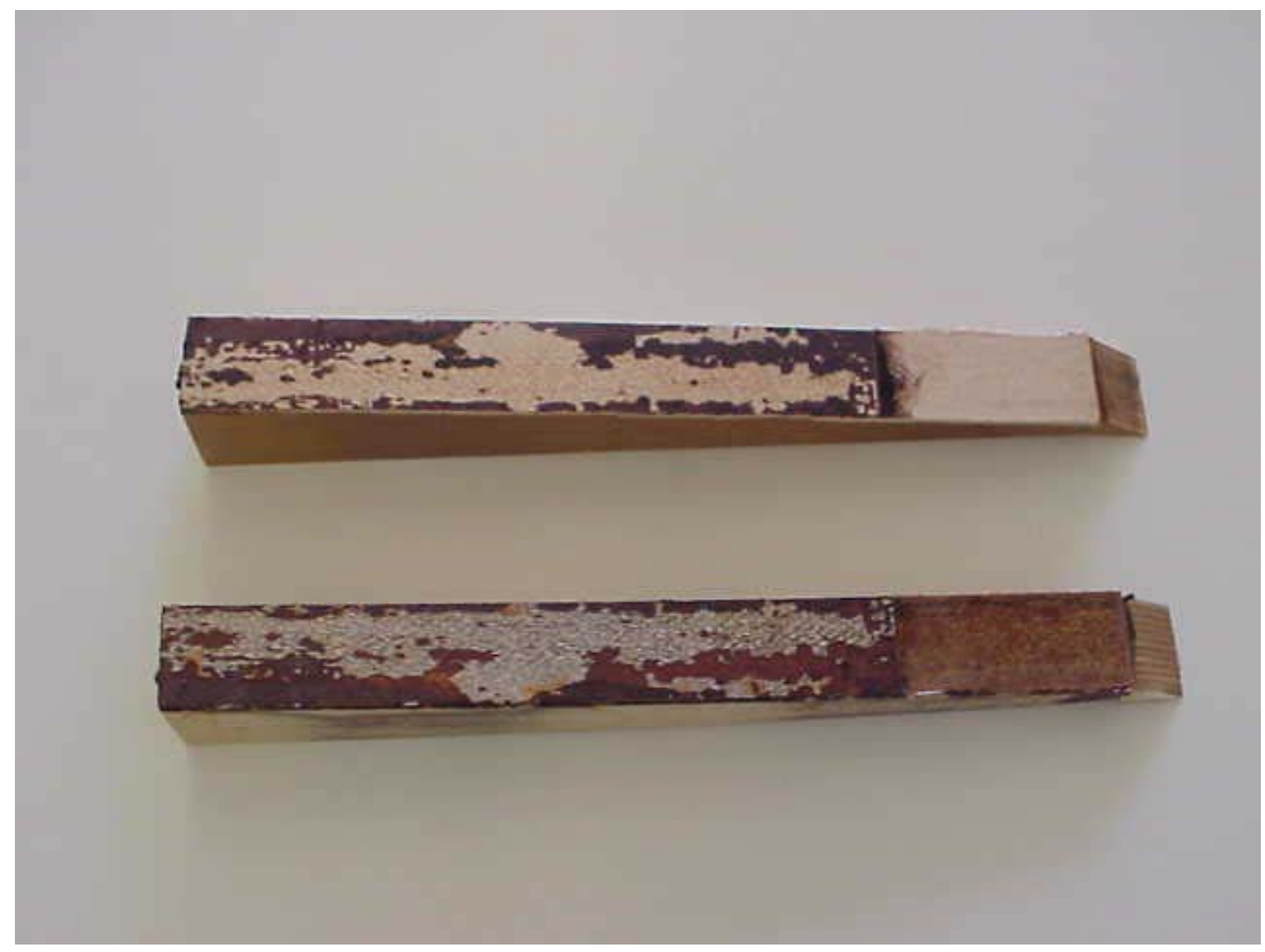

Fig. 3.46 Fractured faces for a CDCB specimen under 27-90 lb. cyclic loading with a frequency $f=1 \mathrm{~Hz}$, load ratio $R=0.3$ and sinusoidal waveform 


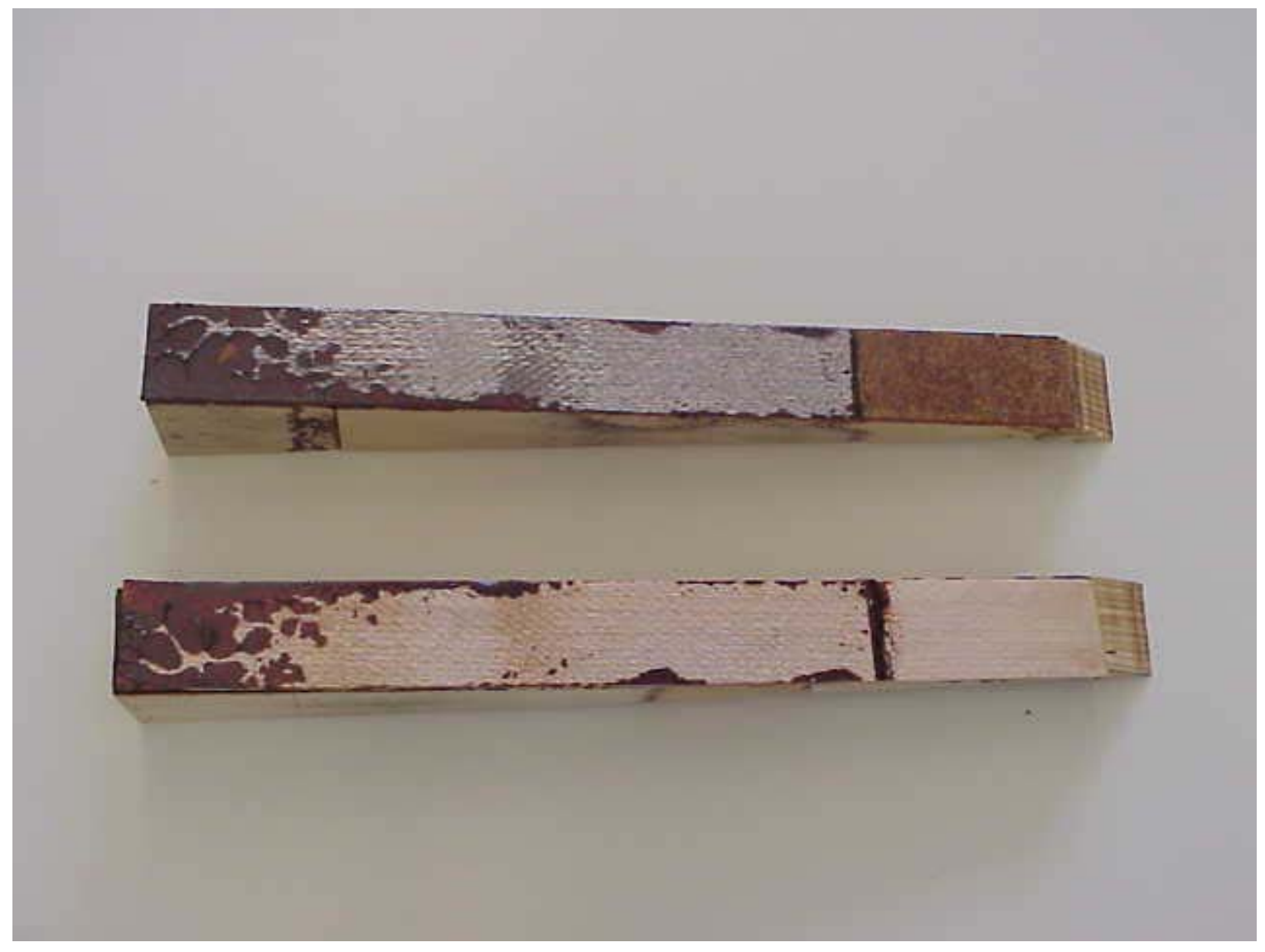

Fig. 3.47 Fractured faces for a CDCB specimen under 30-100 lb. cyclic loading with a frequency $f=1 \mathrm{~Hz}$, load ratio $R=0.3$ and sinusoidal waveform 


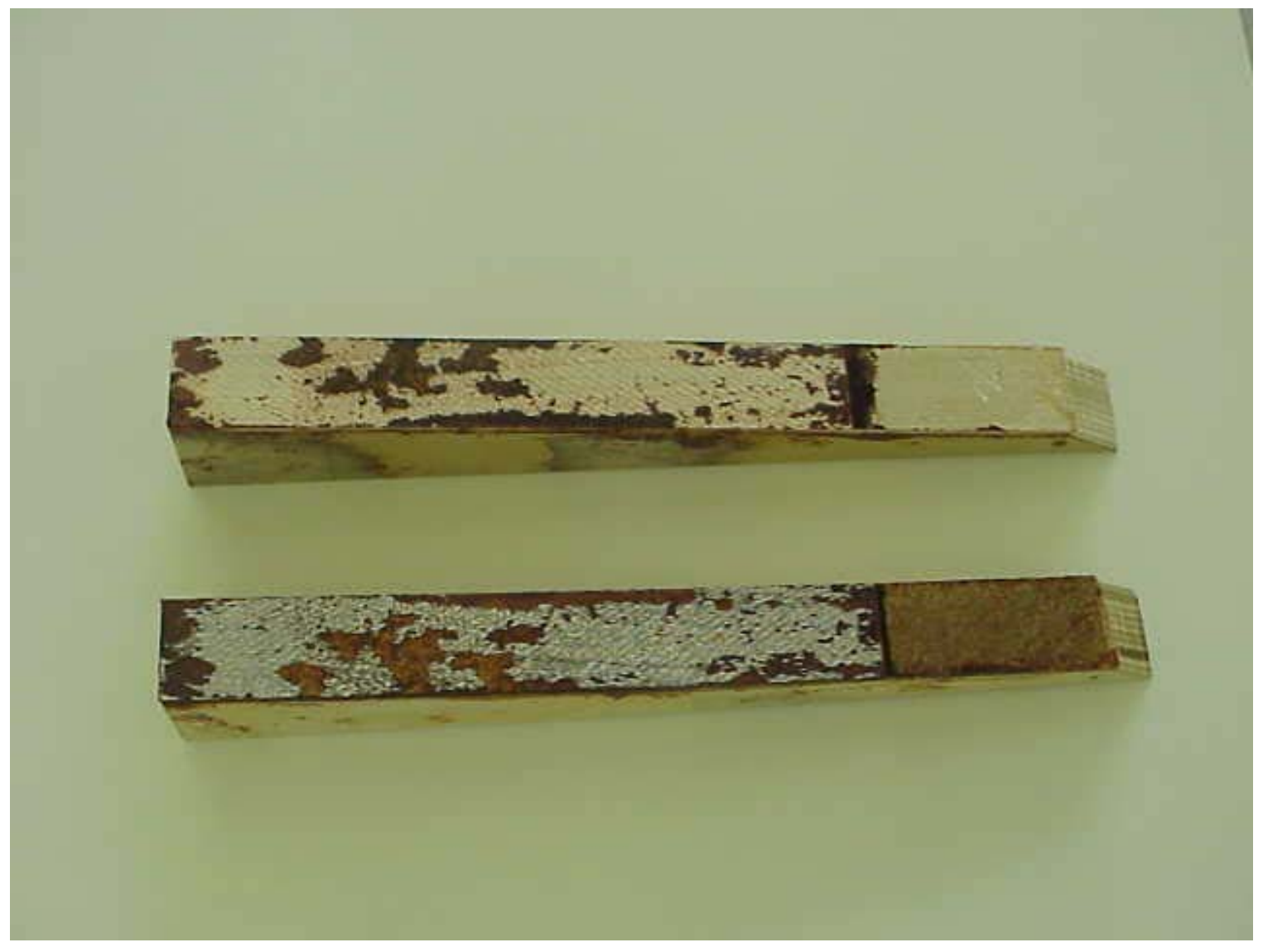

Fig. 3.48 Fractured faces for a CDCB specimen under 33-110 lb. cyclic loading with a frequency $f=1 \mathrm{~Hz}$, load ratio $R=0.3$ and sinusoidal waveform 


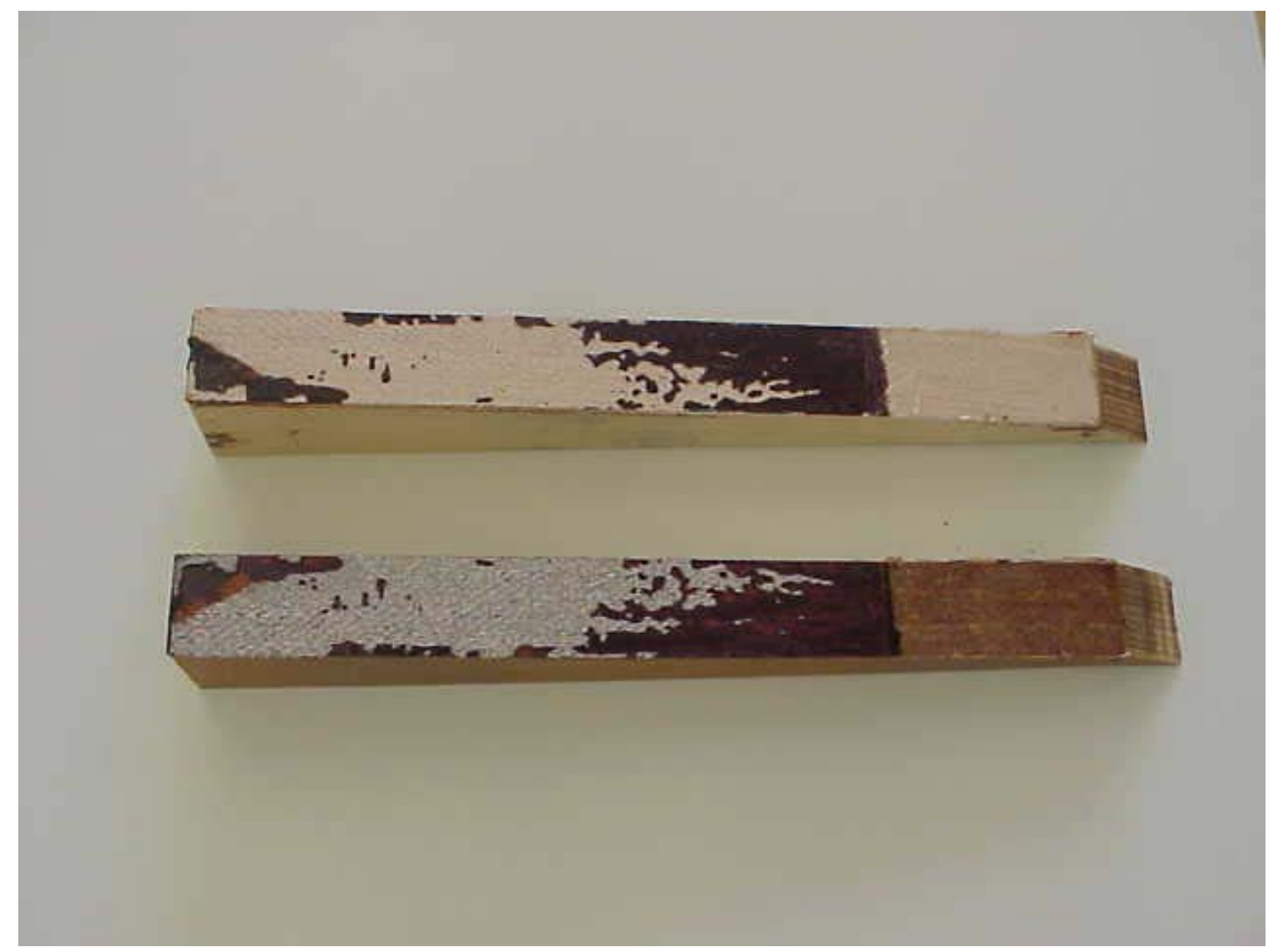

Fig. 3.49 Fractured faces for a CDCB specimen under 36-120 lb. cyclic loading with a frequency $f=1 \mathrm{~Hz}$, load ratio $R=0.3$ and sinusoidal waveform 


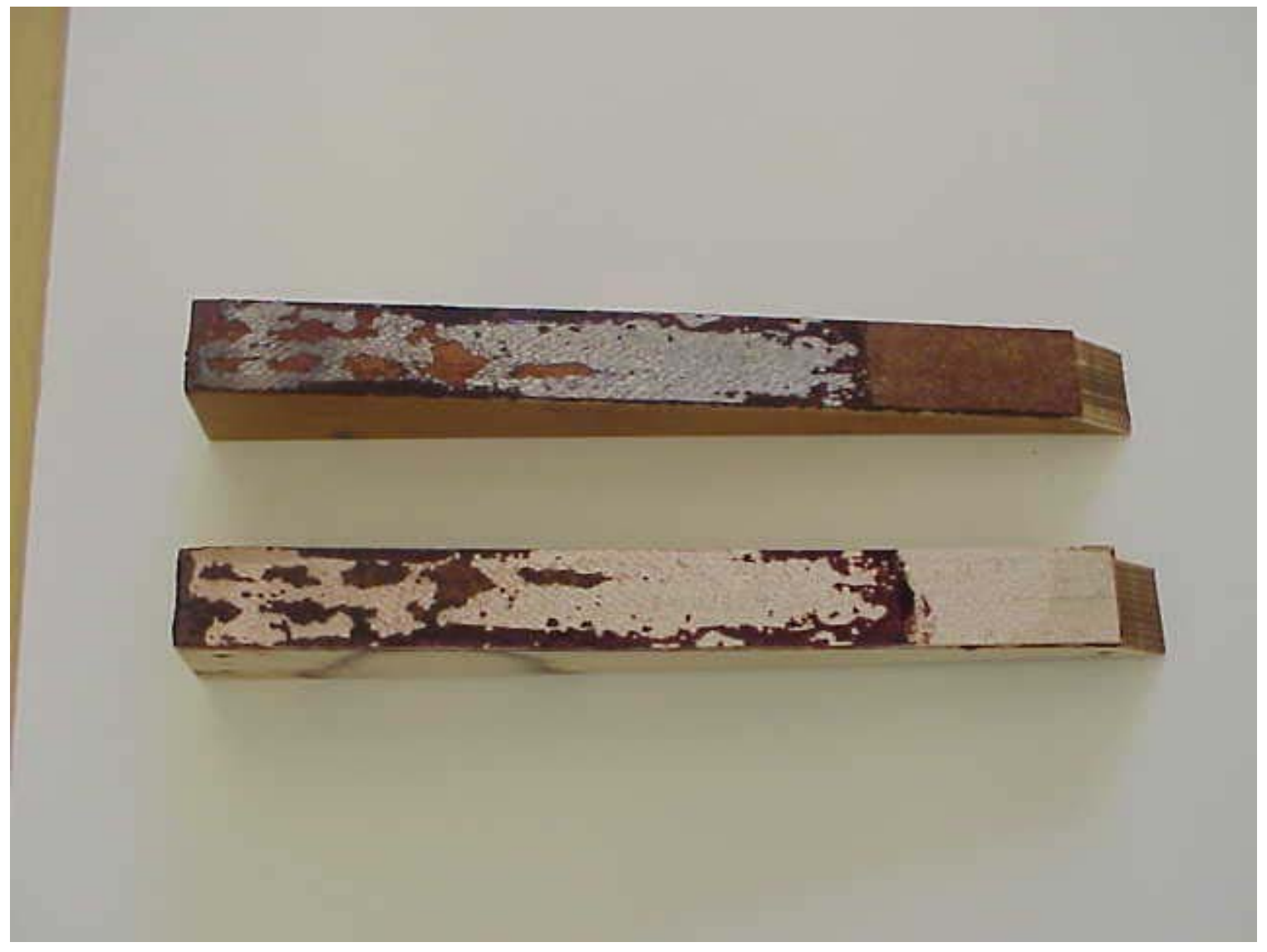

Fig. 3.50 Fractured faces for a CDCB specimen under 37.5-125 lb. cyclic loading with a frequency $f=1 \mathrm{~Hz}$, load ratio $R=0.3$ and sinusoidal waveform 


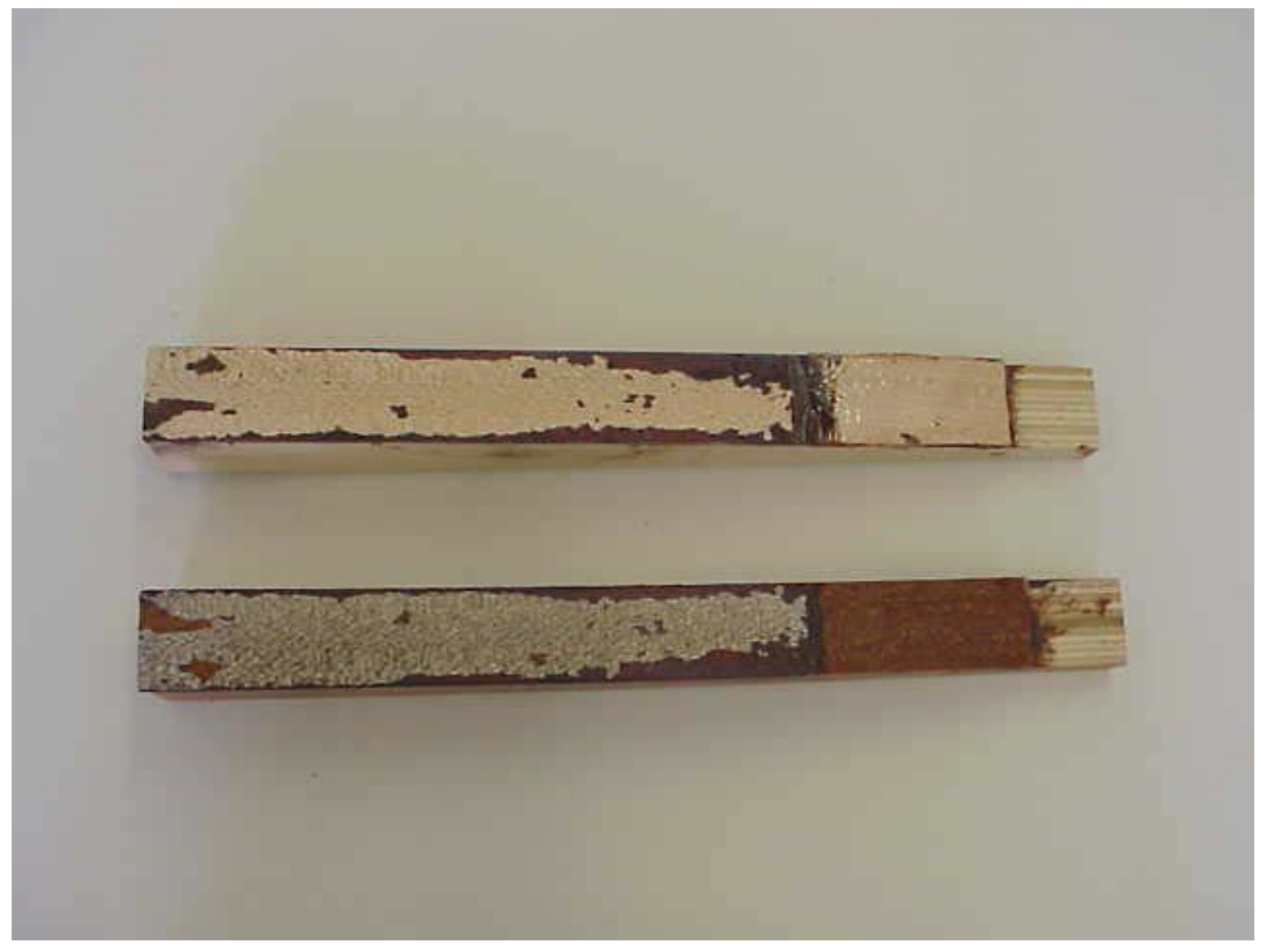

Fig. 3.51 Fractured faces for a CDCB specimen under 39-130 lb. cyclic loading with a frequency $f=1 \mathrm{~Hz}$, load ratio $R=0.3$ and sinusoidal waveform 


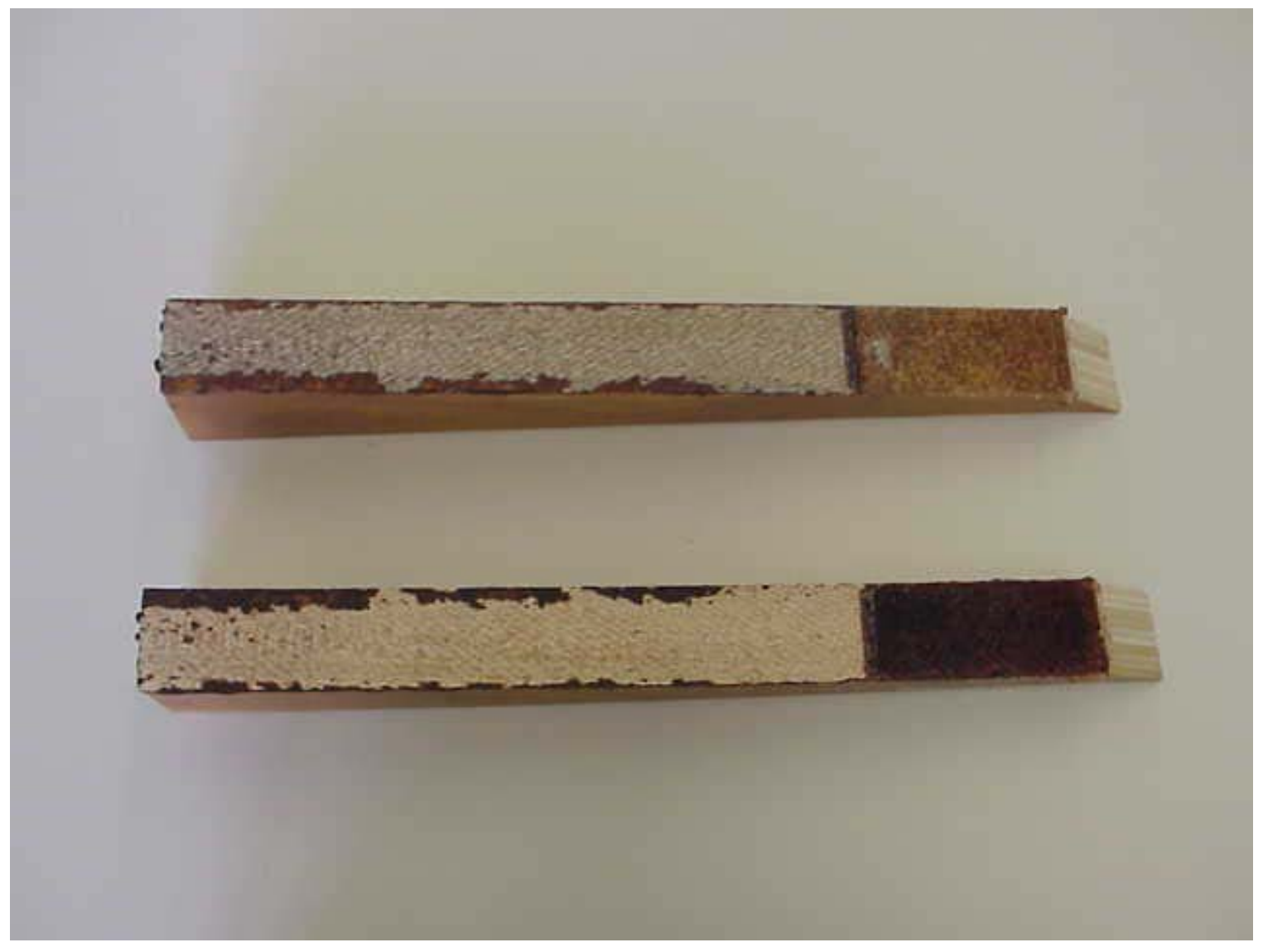

Fig. 3.52 Fractured faces for a CDCB specimen under 40-80 lb. cyclic loading with a frequency $f=1 \mathrm{~Hz}$, load ratio $R=0.5$ and sinusoidal waveform 


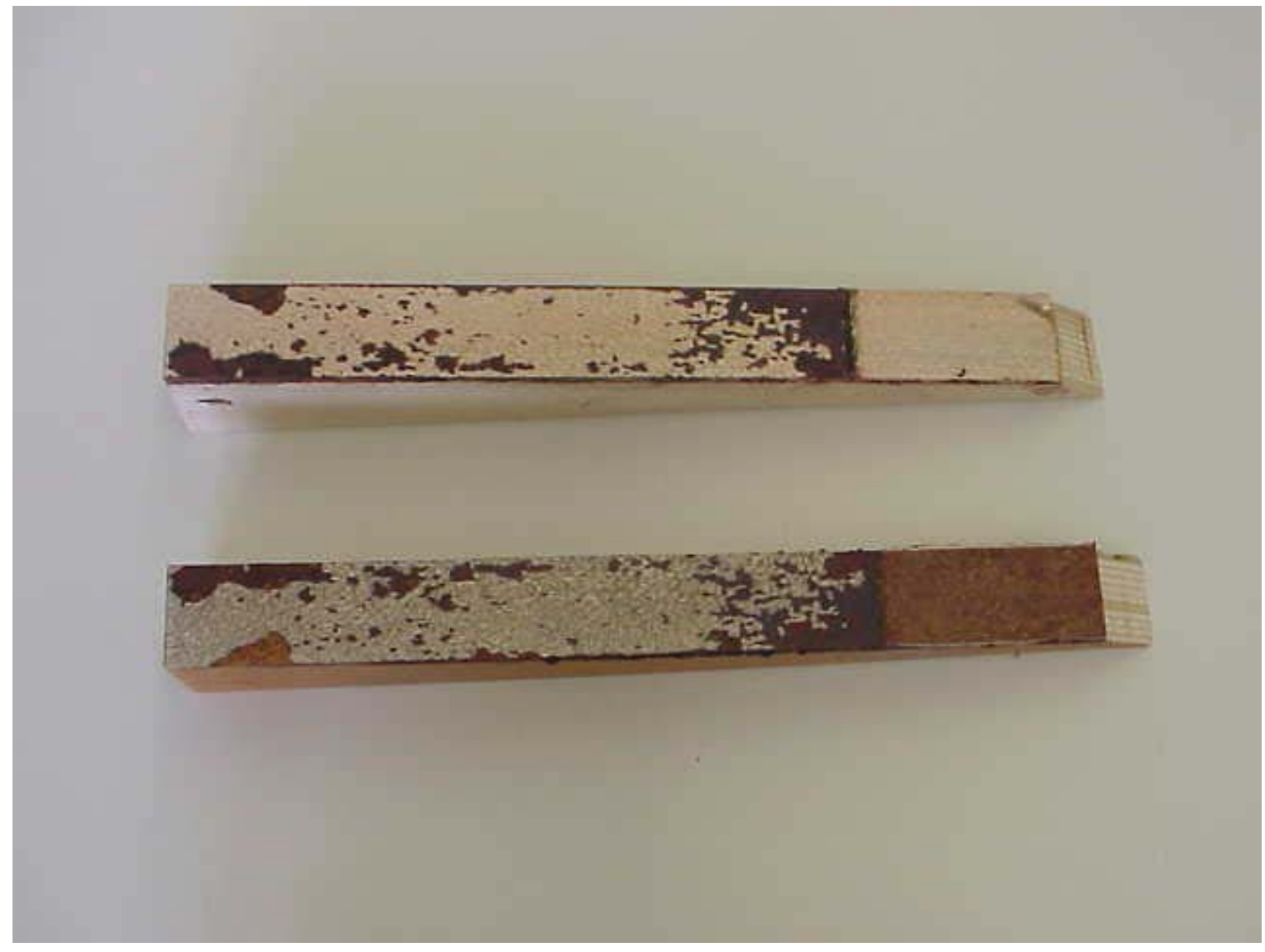

Fig. 3.53 Fractured faces for a CDCB specimen under 42.5-85 lb. cyclic loading with a frequency $f=1 \mathrm{~Hz}$, load ratio $R=0.5$ and sinusoidal waveform 


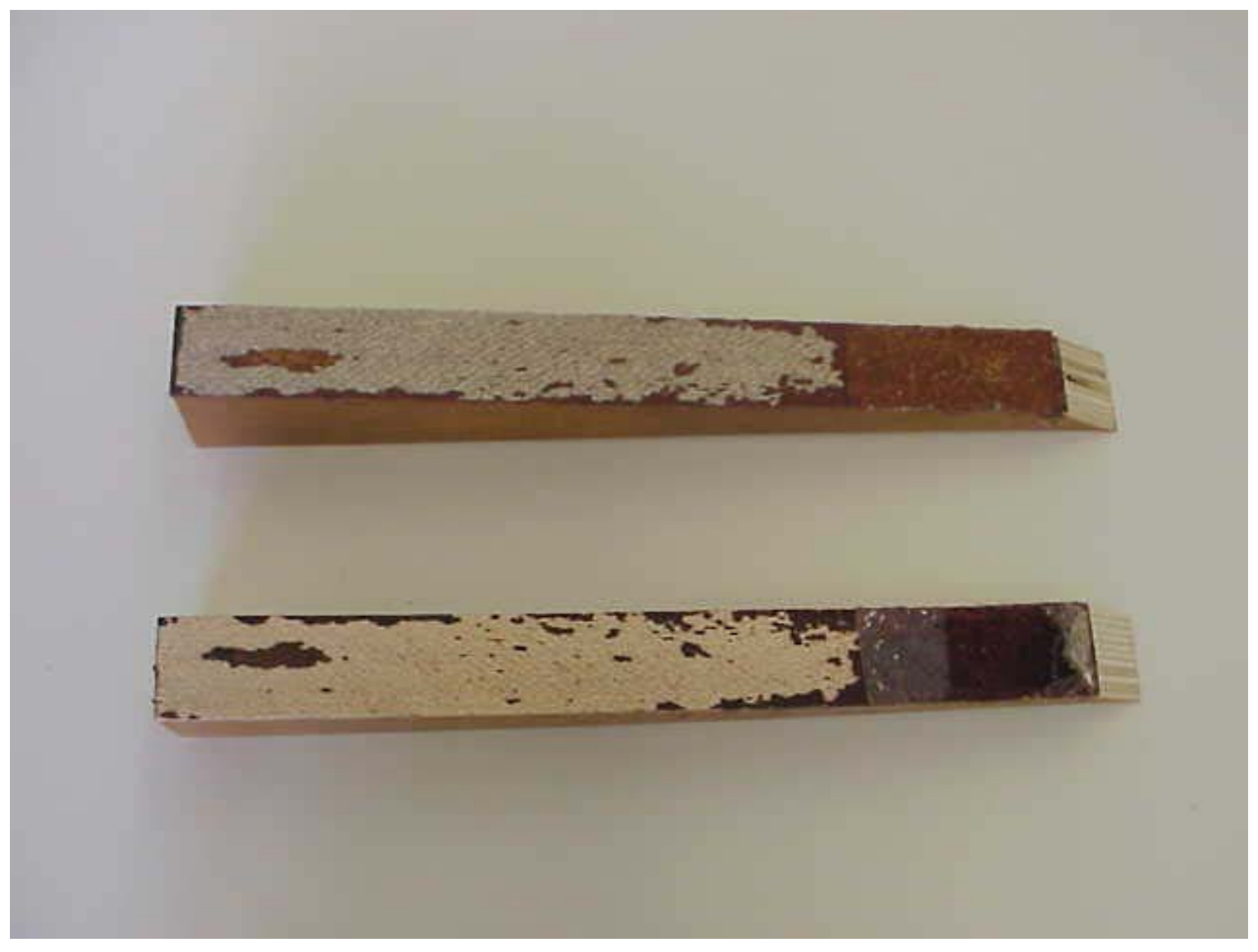

Fig. 3.54 Fractured faces for a CDCB specimen under 45-90 lb. cyclic loading with a frequency $f=1 \mathrm{~Hz}$, load ratio $R=0.5$ and sinusoidal waveform 


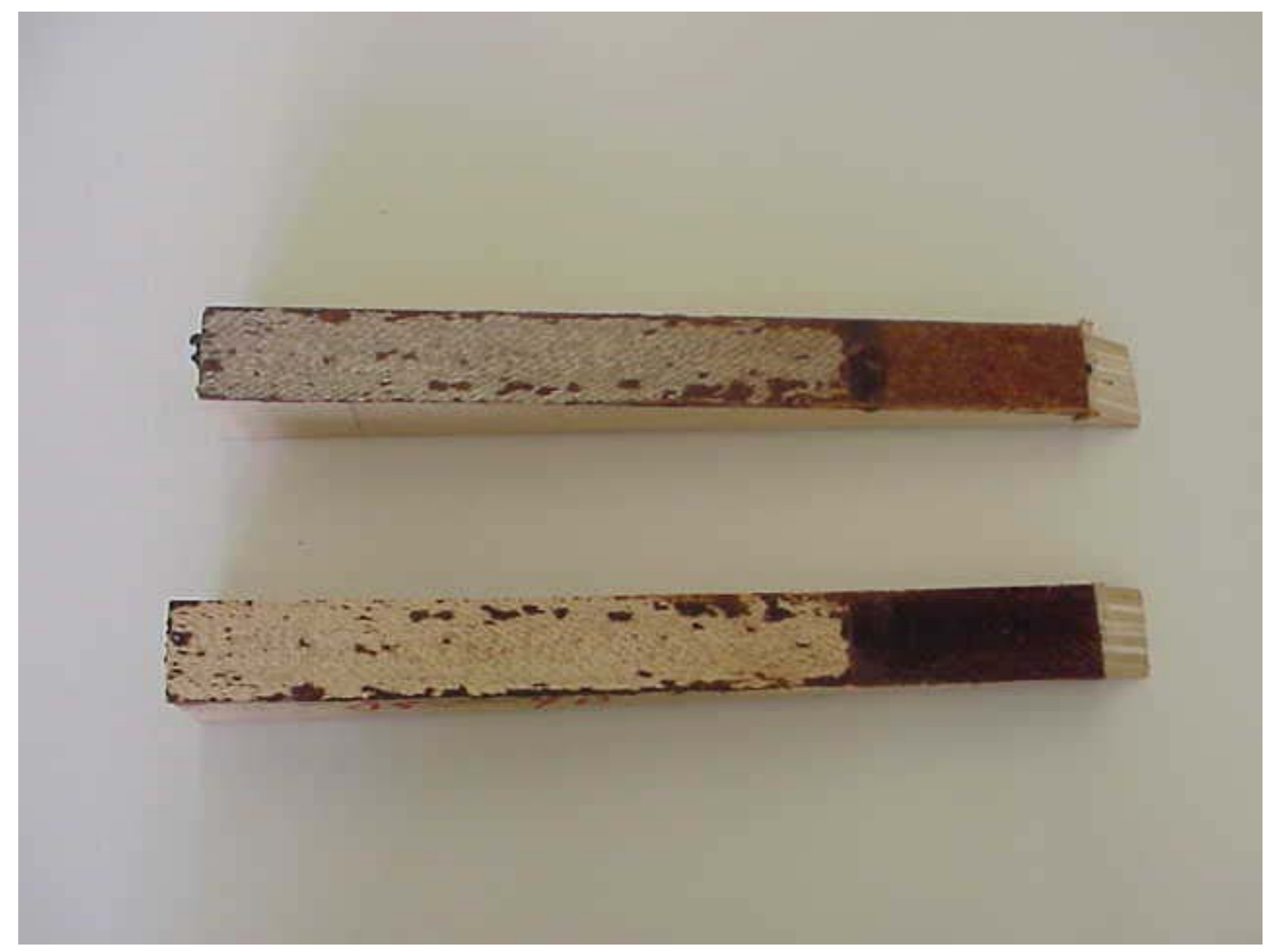

Fig. 3.55 Fractured faces for a CDCB specimen under 47.5-95 lb. cyclic loading with a frequency $f=1 \mathrm{~Hz}$, load ratio $R=0.5$ and sinusoidal waveform 


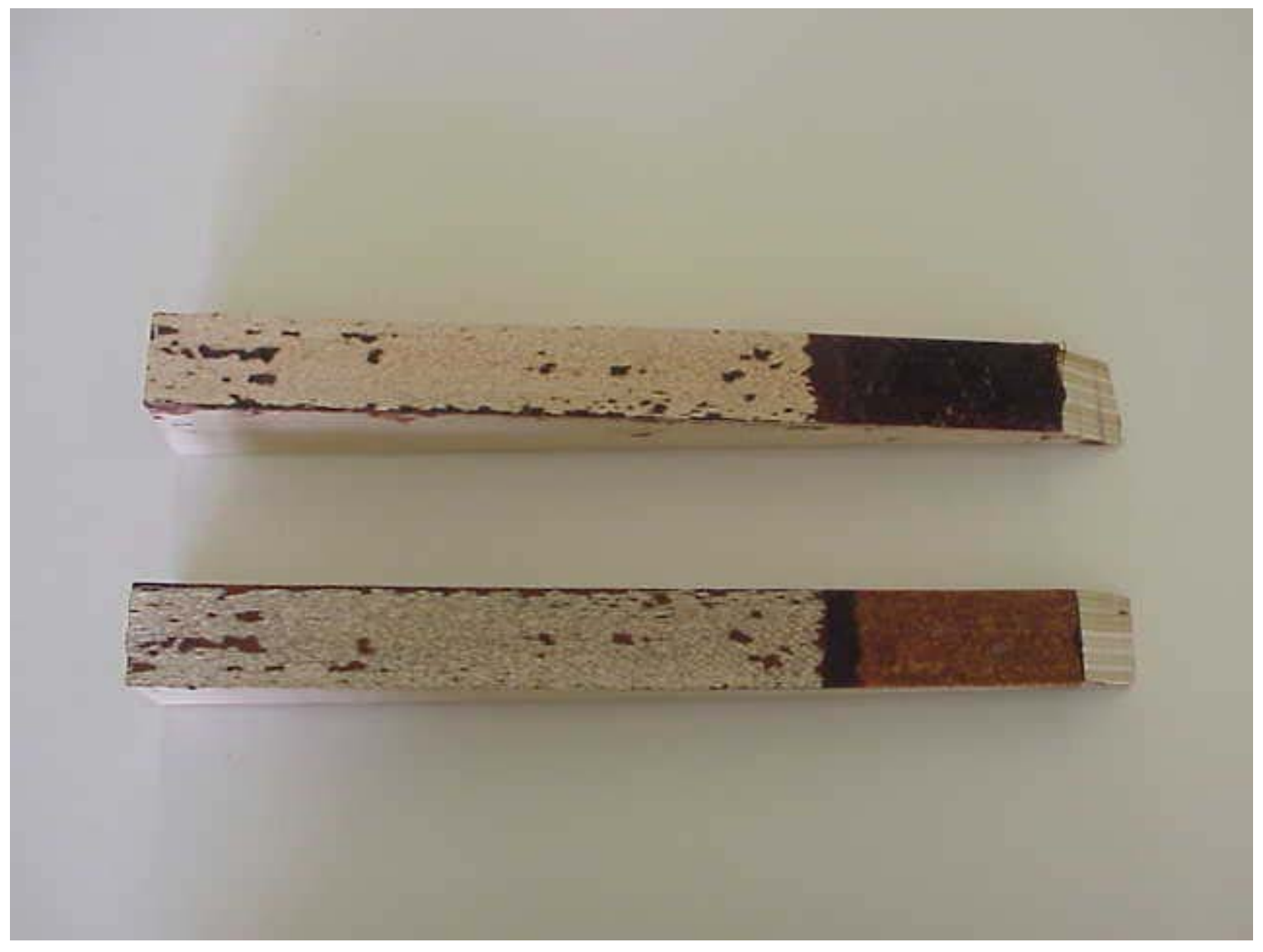

Fig. 3.56 Fractured faces for a CDCB specimen under 50-100 lb. cyclic loading with a frequency $f=1 \mathrm{~Hz}$, load ratio $R=0.5$ and sinusoidal waveform 


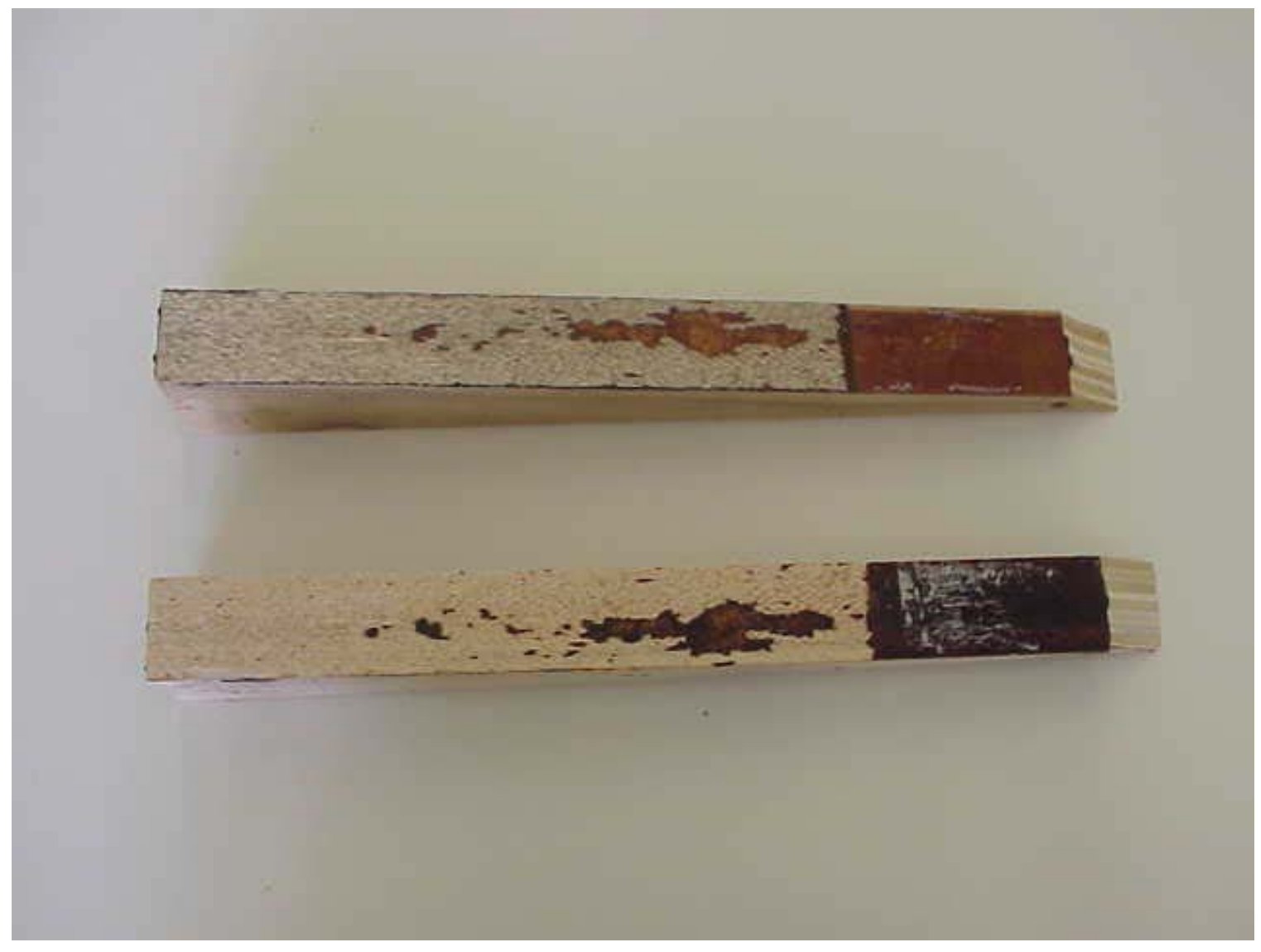

Fig. 3.57 Fractured faces for a CDCB specimen under 55-110 lb. cyclic loading with a frequency $f=1 \mathrm{~Hz}$, load ratio $R=0.5$ and sinusoidal waveform 


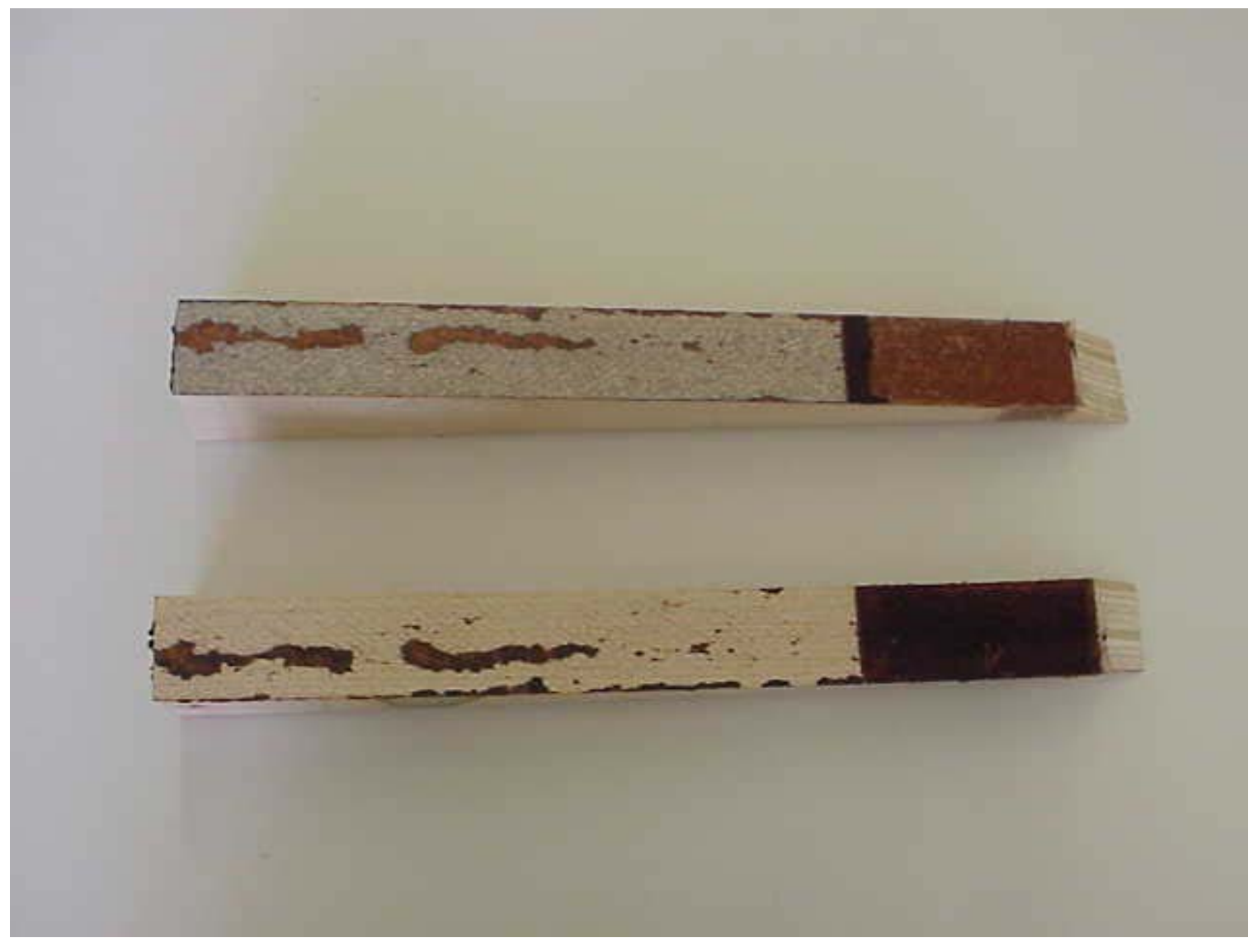

Fig. 3.58 Fractured faces for a CDCB specimen under 60-120 lb. cyclic loading with a frequency $f=1 \mathrm{~Hz}$, load ratio $R=0.5$ and sinusoidal waveform 


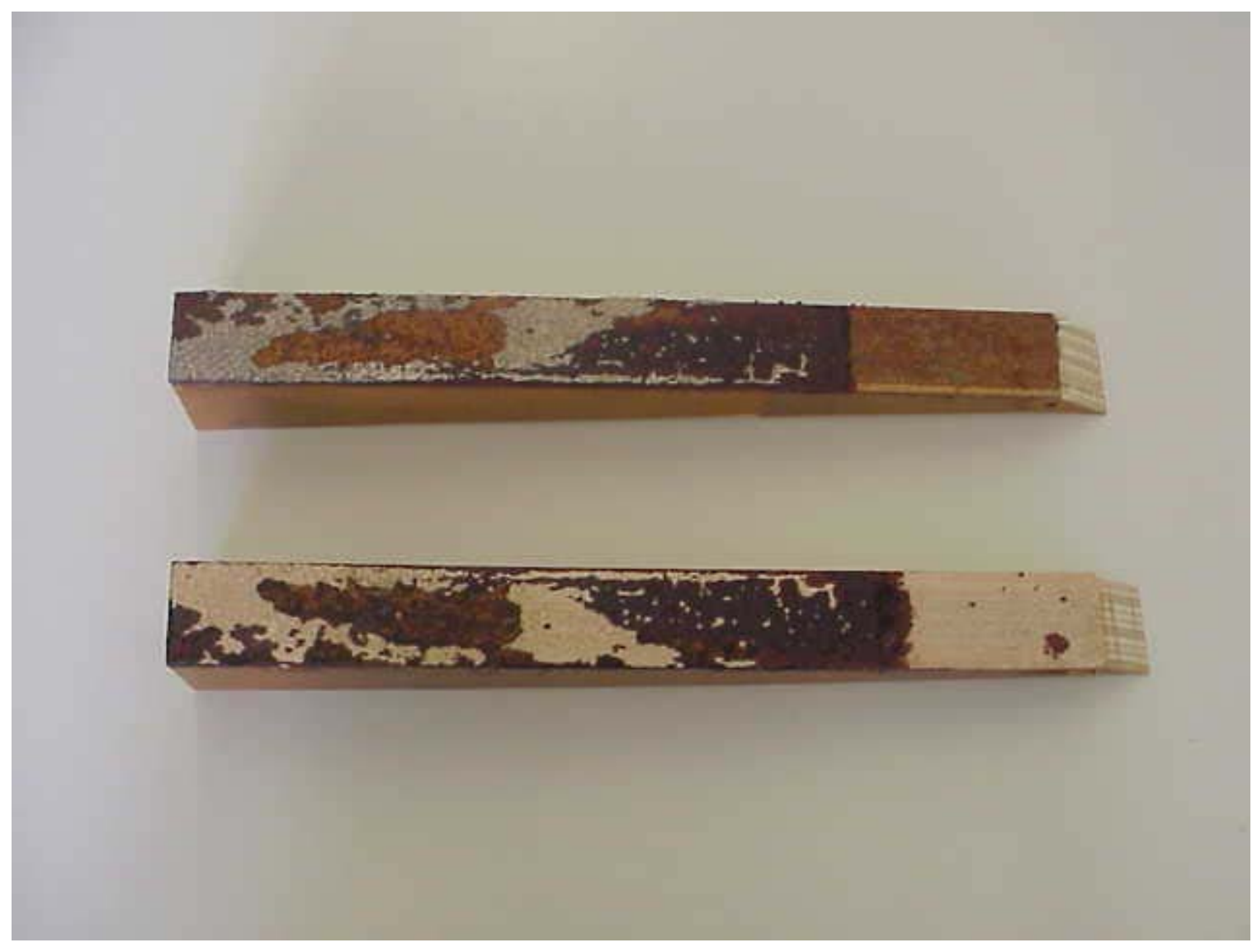

Fig. 3.59 Fractured faces for a CDCB specimen under 65-130 lb. cyclic loading with a frequency $f=1 \mathrm{~Hz}$, load ratio $R=0.5$ and sinusoidal waveform 


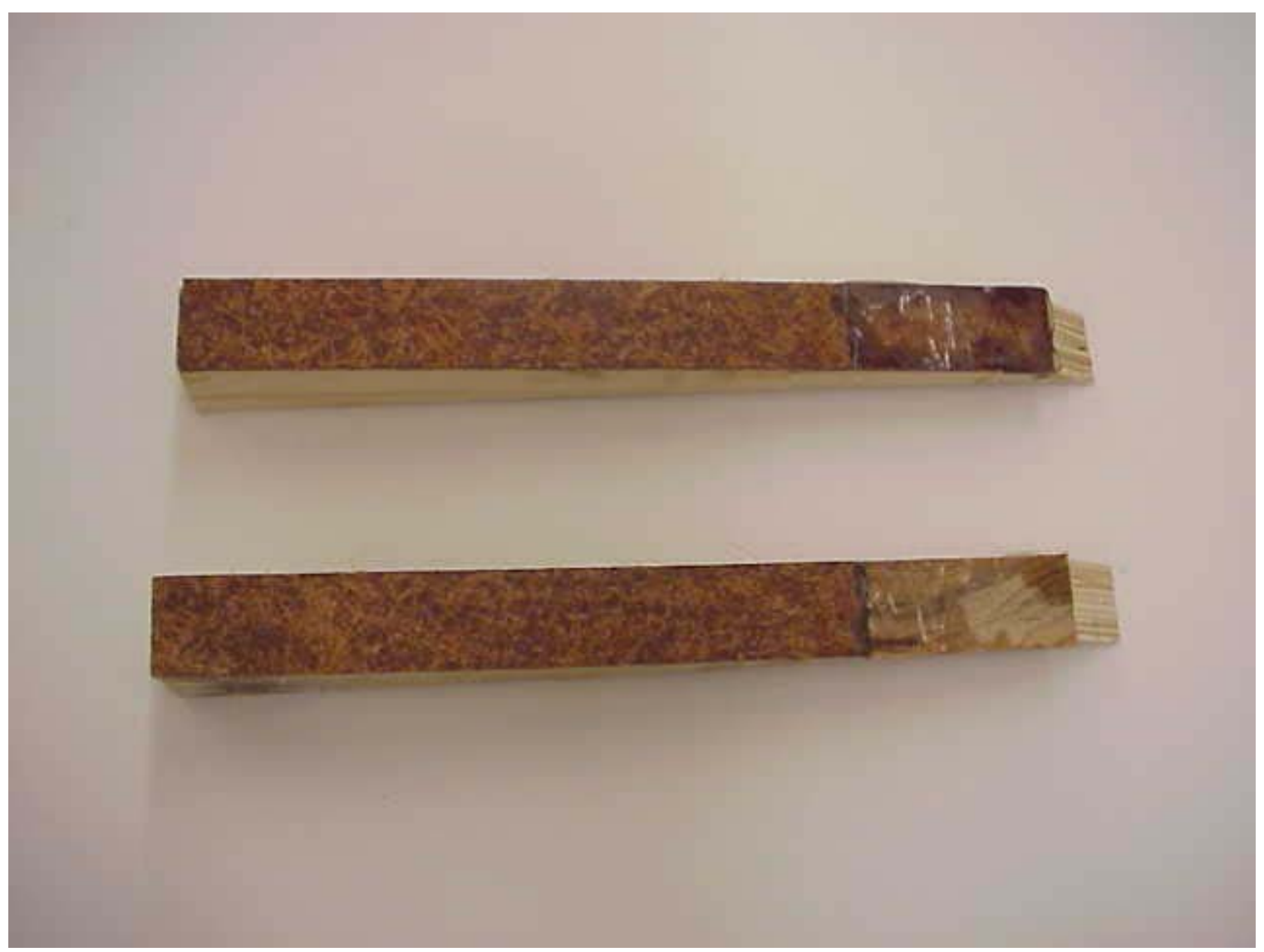

Fig. 3.60 Fractured faces having fiber bridging for a CDCB specimen under 65-130 lb. cyclic loading with a frequency $f=1 \mathrm{~Hz}$, load ratio $R=0.5$ and sinusoidal waveform 


\subsection{Conclusions}

The load ratio effect on the crack propagation rate of the interface bond between red maple wood and phenolic FRP composite is studied in this chapter. By using a contoured double cantilever beam (CDCB) specimen corresponding to constant strain energy release rate range $\Delta G$, three sets of tests of load ratio $0.1,0.3$ and 0.5 are performed. All the tests are carried out under constant amplitude cyclic loading at room temperature and laboratory conditions. A modified Paris Law equation is proposed to explain the load ratio effect, and a discussion on crack closure effect, which may provide a physical explanation for load ratio effect, is presented. Also a discussion of the failure surfaces is given. The results of this chapter are summarized as follows:

1. The CDCB specimen is shown to be suitable for evaluating red maple woodphenolic FRP composite interface fatigue behavior. The hybrid interface bond displayed a typical crack propagation behavior under constant cyclic loading as observed by others for other materials.

2. The compliance method used for the CDCB specimen is quite simple, relatively precise and effective for measuring crack propagation rate of red maple woodphenolic FRP composite interfaces.

3. A catastrophic ultimate failure of the interface under constant cyclic loading is observed in this study. 
4. The Paris Law equation using strain energy release rate as a controlling parameter can be efficiently applied for a given value of load ratio $R$ :

$$
\frac{d a}{d N}=B(\Delta G)^{m}
$$

where $B$ and $m$ are material constants, which are strongly dependent on load ratio $R$. Both $B$ and $m$ increase as the load ratio $R$ increases.

5. Any two of the following parameters: $G_{m i n}, G_{m e a n}$, and $R$ combined with $\Delta G$ can characterize crack growth rate under different load ratios. The modified Paris Law equations for the red maple wood-phenolic FRP composite interface bond considered in this chapter are given as

$$
\begin{aligned}
& \frac{d a}{d N}=2 \times 10^{-6}\left(\frac{\Delta G}{(1-R)^{0.79}}\right)^{5.54} \\
& \frac{d a}{d N}=7 \times 10^{-5}\left(\Delta G^{0.87} G_{\text {min }}^{0.13}\right)^{5.34} \\
& \frac{d a}{d N}=1 \times 10^{-4}\left(\Delta G^{0.07} G_{\text {mean }}^{0.93}\right)^{5.59}
\end{aligned}
$$

Any of these proposed equations can efficiently represent the load ratio effect on crack propagation rate of wood-FRP interfaces, and they can further be used for other similar studies of interface fracture of dissimilar materials. 
6. The modified Paris Law equation

$$
\frac{d a}{d N}=1 \times 10^{-4}\left(\Delta G^{0.07} G_{\text {mean }}^{0.93}\right)^{5.59}
$$

can best explain the load ratio effect for red maple wood-phenolic FRP composite interface fatigue behavior. We strongly recommend this equation for engineering applications.

7. The crack closure effect is observed for the phenolic FRP-red maple wood bonded interface under tension-tension cyclic loading, especially for load ratio of 0.1 .

8. The crack closure may provide a physical explanation for the load ratio effect on the crack propagation rate of red maple wood-phenolic FRP bonded interfaces.

9. The relationship between effective or equivalent strain energy release rate range ratio $\alpha$ and load ratio $R$ can be expressed as

$$
\alpha=\frac{R}{1-R}+0.5
$$

This equation gives a good estimation of $\alpha$ as a function of load ratio $R$. 
10. The critical load ratio $R_{\text {critical }}$ corresponding to the effective or equivalent strain energy release rate range ratio $\alpha$ equal to 1 is approximately equal to 0.33 .

11. When crack closure is included, $\Delta G$ combine any of the following parameters: $R$, $G_{m i n}, G_{m e a n}$ and $\alpha$ can explain the load ratio effect.

12. The interface bond failure is the primary failure mode for the phenolic FRP-red maple wood CDCB specimens tested here. 


\section{CHAPTER 4}

\section{WAVEFORM EFFECT}

\subsection{Introduction}

Fatigue fracture for metals has been extensively explored. A fundamental principle of crack propagation established by Paris, Gomez and Anderson (1961) and Paris and Erdogan (1963) stated that for a crack length $a$ and number of cycles $N$, the crack growth rate, $d a / d N$, depends on the amplitude of the cyclic stress intensity factor $\Delta K$. This dependence is approximately expressed as a power function, or Paris Law equation, as

$$
\frac{d a}{d N}=C \Delta K^{m}
$$

where, $C$ and $m$ are empirical constants that depend on material, frequency, load ratio, temperature, environment and other factors. By incorporating elastic fracture mechanics concepts, the Paris Law equation has been successful in explaining crack growth rate and predicting fatigue life. However, many questions remained, such as the effects of waveform, load ratio, and frequency on fatigue behavior related to crack growth rate. To address these concerns, a number of investigators have proposed modifications to the Paris Law equation to account for such effects. 
The load ratio effect on crack propagation rate of red maple wood-phenolic FRP composite bonded interfaces has been studied in Chapter 3. A modified Paris Law equation has been proposed to explain the load ratio effect. In this chapter, the waveform effect on crack propagation rate of red maple wood-phenolic FRP composite bonded interfaces is investigated. The contents of this chapter include: (1) Literature review, (2) Fatigue tests, (3) Results, and (4) Conclusions.

\subsection{Literature Review}

For waveform effect, there are only a few studies in the literature. Tong and Byrne (1999) tested a pre-alloyed powder material Udimet 720Li and found that the crack growth rate results for triangular and trapezoidal waveforms were almost the same, except at a frequency less than $0.01 \mathrm{~Hz}$, for which the crack growth rate increased significantly. However, most researchers support the notion that waveform effect is not of concern and can be neglected.

\subsection{Fatigue Test}

This section describes materials, test specimen, and procedures.

\subsubsection{Materials and specimen}

A contoured double cantilever beam (CDCB) specimen designed by the RayleighRitz method (see section 1.5.2) was used for waveform effect study of red maple wood- 
phenolic FRP composite bonded interfaces. The adherends consist of red maple wood and pultruded phenolic FRP with the contoured portions made of laminated veneer lumber (LVL). The adhesive used for bonding the red maple wood-phenolic FRP bonded interface was Resorcinol-Formaldehyde from INDSPEC Chemical Corp., Pittsburgh, PA. The lay-up of the pultruded phenolic FRP laminate is shown in Figure 2.3, and the mechanical properties of red maple, LVL and phenolic FRP are summarized in Table 2.2. The CDCB specimen is contoured to achieve a constant rate of compliance change with respect to crack length, $d C / d a$. The details of the specimen design and applications for wood-FRP interface fracture studies are given in Chapter 1 and Chapter 2. The geometric details of the CDCB specimen are shown in Fig. 2.4.

The average of the $d C / d a$ value obtained by the Rayleigh-Ritz method and finite element modeling was $27.17 \times 10^{-5} \mathrm{lb}^{-1}$. Compliance calibration tests were conducted for several wood-FRP CDCB samples, and the average $d C / d a$ value obtained from experiments was $29.59 \times 10^{-5} \mathrm{lb}^{-1}$. The experimental result of the $d C / d a$ value is used to calculate strain energy release rate $G$ in the present study.

\subsubsection{Test procedure}

Mode-I fatigue tests were conducted in an MTS servo hydraulic testing machine. The cyclic load was applied by using a loading fixture made of aluminum (see Fig. 2.9). Three groups of tests for loading shapes of sinusoidal waveform, triangular waveform, and square waveform were completed under load control mode (see Fig. 4.1). The waveform was kept the same during the testing of each group of samples. The applied 
loads for each group of tests are listed in Tables 4.1, 4.2 and 4.3. A frequency of $1 \mathrm{~Hz}$ and a load ratio $R$ (the ratio of minimum load to the maximum load) of 0.5 were used for all the tests. The tests in the MTS machine were controlled by pre-programmed software GPA, which was also used as a data acquisition system. Since only the relative value of crack opening displacement (COD) is needed, a crosshead displacement instead of an extensometer displacement was used to measure the COD. The test environment was room temperature and open laboratory conditions for all the samples.

Table 4.1 Applied load for the triangular waveform

\begin{tabular}{|c|c|c|c|c|}
\hline $\boldsymbol{P}_{\min }$ & $\boldsymbol{P}_{\max }$ & $\Delta \boldsymbol{P}=\boldsymbol{P}_{\max }-\boldsymbol{P}_{\min }$ & $\boldsymbol{R}$ & $\boldsymbol{P}_{\text {mean }}$ \\
\hline 65 & 130 & 65 & 0.5 & 97.5 \\
\hline 60 & 120 & 60 & 0.5 & 90 \\
\hline 55 & 110 & 55 & 0.5 & 82.5 \\
\hline 50 & 100 & 32.5 & 0.5 & 75 \\
\hline 45 & 90 & 45 & 0.5 & 67.5 \\
\hline 40 & 80 & 40 & 0.5 & 60 \\
\hline
\end{tabular}


Table 4.2 Applied load for the sinusoidal waveform

\begin{tabular}{|c|c|c|c|c|}
\hline $\boldsymbol{P}_{\min }$ & $\boldsymbol{P}_{\max }$ & $\Delta \boldsymbol{P}=\boldsymbol{P}_{\max }-\boldsymbol{P}_{\min }$ & $\boldsymbol{R}$ & $\boldsymbol{P}_{\text {mean }}$ \\
\hline 65 & 130 & 65 & 0.5 & 97.5 \\
\hline 60 & 120 & 60 & 0.5 & 90 \\
\hline 55 & 110 & 55 & 0.5 & 82.5 \\
\hline 50 & 100 & 32.5 & 0.5 & 75 \\
\hline 47.5 & 95 & 47.5 & 0.5 & 71.25 \\
\hline 45 & 90 & 45 & 0.5 & 67.5 \\
\hline 42.5 & 85 & 42.5 & 0.5 & 63.75 \\
\hline 40 & 80 & 40 & 0.5 & 60 \\
\hline
\end{tabular}

Table 4.3 Applied load for the square waveform

\begin{tabular}{|c|c|c|c|c|}
\hline $\boldsymbol{P}_{\text {min }}$ & $\boldsymbol{P}_{\max }$ & $\Delta \boldsymbol{P}=\boldsymbol{P}_{\max }-\boldsymbol{P}_{\min }$ & $\boldsymbol{R}$ & $\boldsymbol{P}_{\text {mean }}$ \\
\hline 65 & 130 & 65 & 0.5 & 97.5 \\
\hline 60 & 120 & 60 & 0.5 & 90 \\
\hline 55 & 110 & 55 & 0.5 & 82.5 \\
\hline 50 & 100 & 32.5 & 0.5 & 75 \\
\hline 45 & 90 & 45 & 0.5 & 67.5 \\
\hline 40 & 80 & 40 & 0.5 & 60 \\
\hline
\end{tabular}




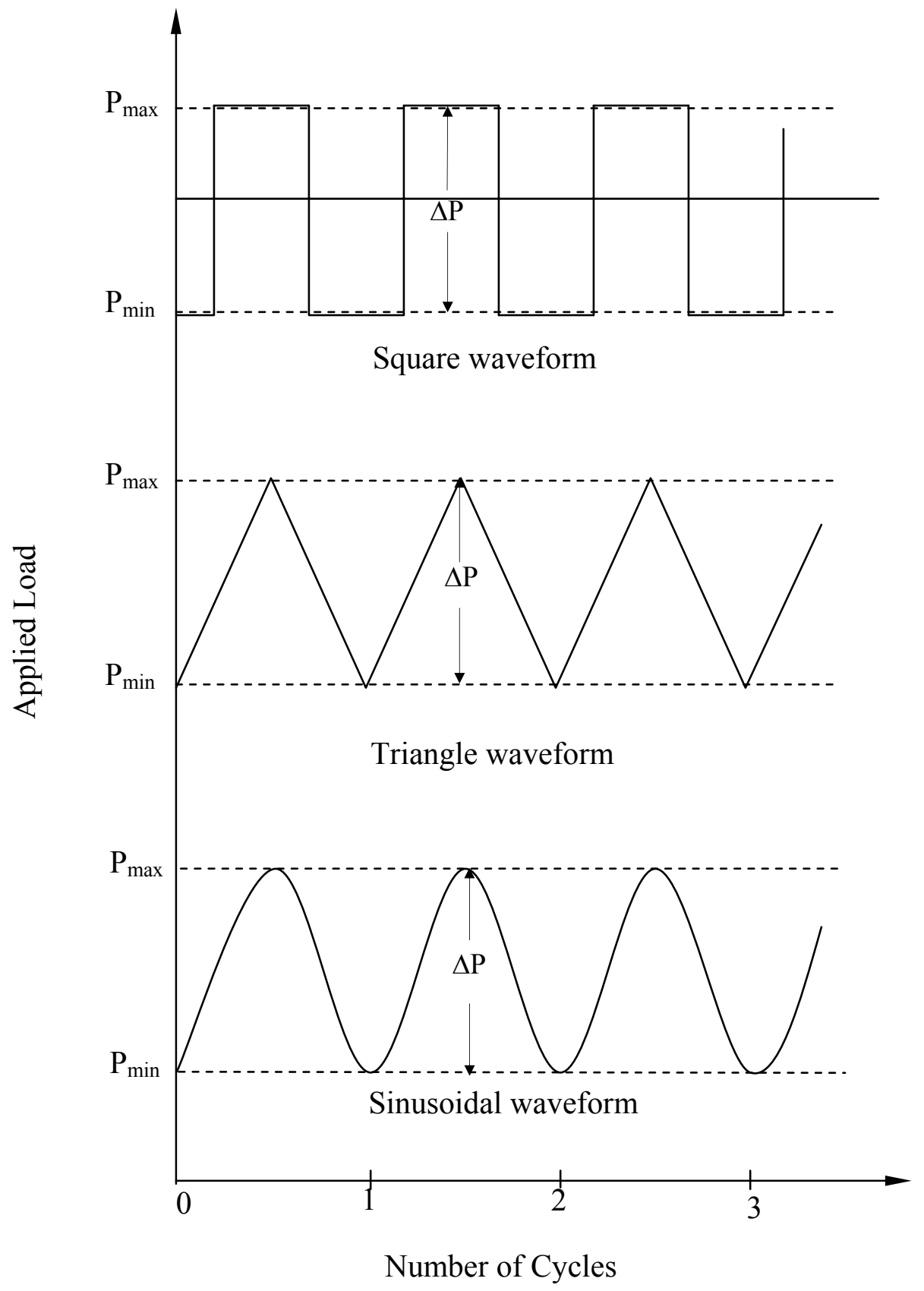

Fig. 4.1 A schematic illustration of cyclic loading for different waveforms 


\subsection{Results}

We present in this section data processing, crack growth rate from compliance method, computation of strain energy release rate parameters, waveform effect on Paris Law and description of failure surfaces.

\subsubsection{Experimental data processing}

The experimental results for the waveform effect on crack propagation rate for phenolic FRP-red maple wood bonded interface samples are summarized and discussed in this section. Following a similar procedure of data processing as in section 3.4.1, diagrams of crack opening displacement (COD) versus number of cycles were produced. For the triangular waveform, Figs. 4.2 through 4.7 display the experimental data of COD versus number of cycles. For the sinusoidal waveform, the same testing was conducted and the previously obtained results for COD versus number of cycles are presented in section 3.4.1. Since fatigue tests are very time consuming and costly, we use the same results reported in Chapter 3 for the sinusoidal waveform for the study described here. For the purpose of comparison, the results for sinusoidal waveform are presented again in a slightly different form in Figs. 4.8 through 4.15. For the square waveform, the relationships of COD versus number of cycles are given in Figs. 4.16 through 4.21. The COD propagation rate $d C O D / d N$ is obtained from linear-regression curve fitting, and the results are listed in Tables 4.4, 4.5 and 4.6. 


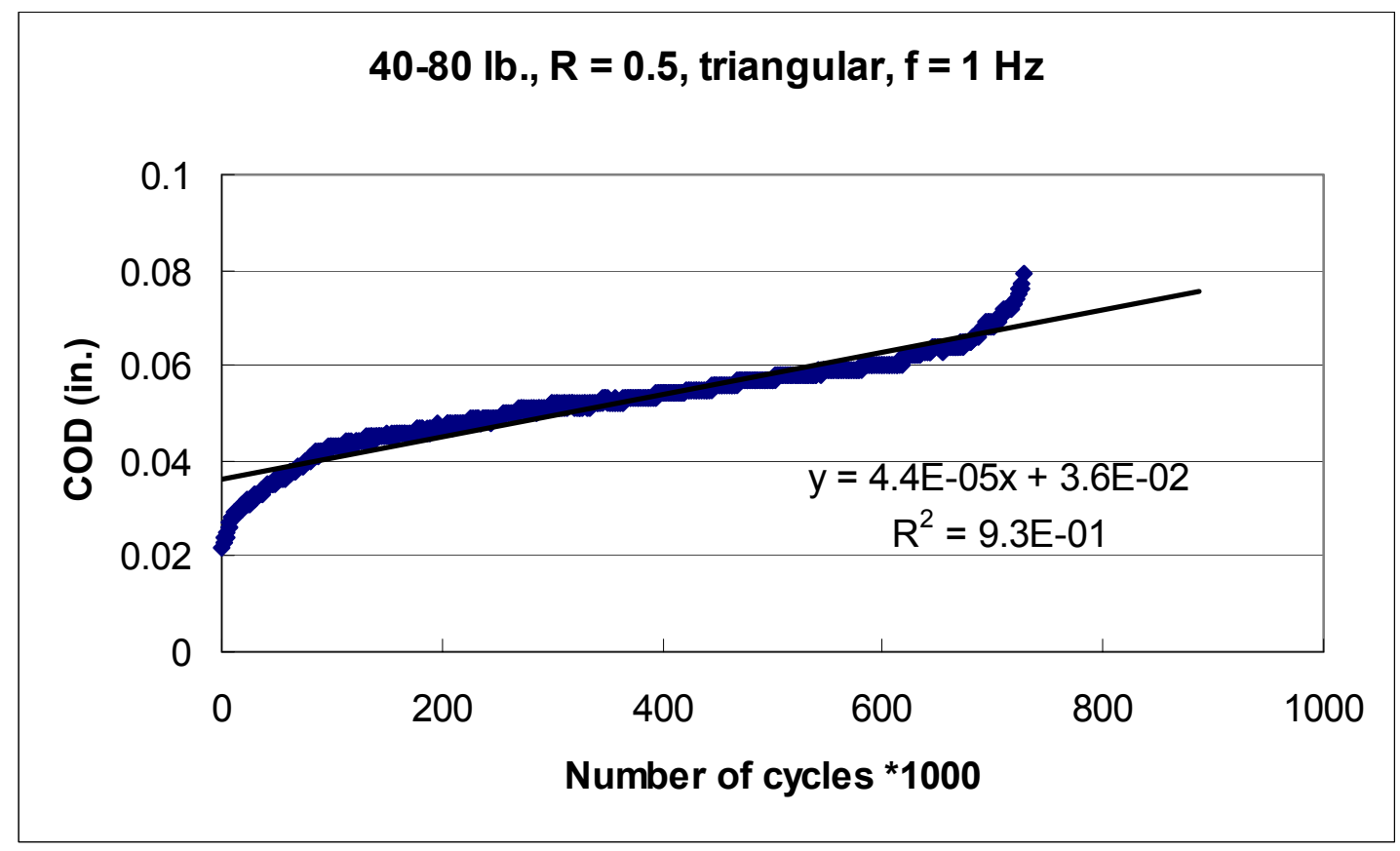

Fig. 4.2 COD versus number of cycles for a CDCB specimen under 40-80 lb. cyclic loading with a frequency $f=1 \mathrm{~Hz}$, load ratio $R=0.5$ and triangular waveform 


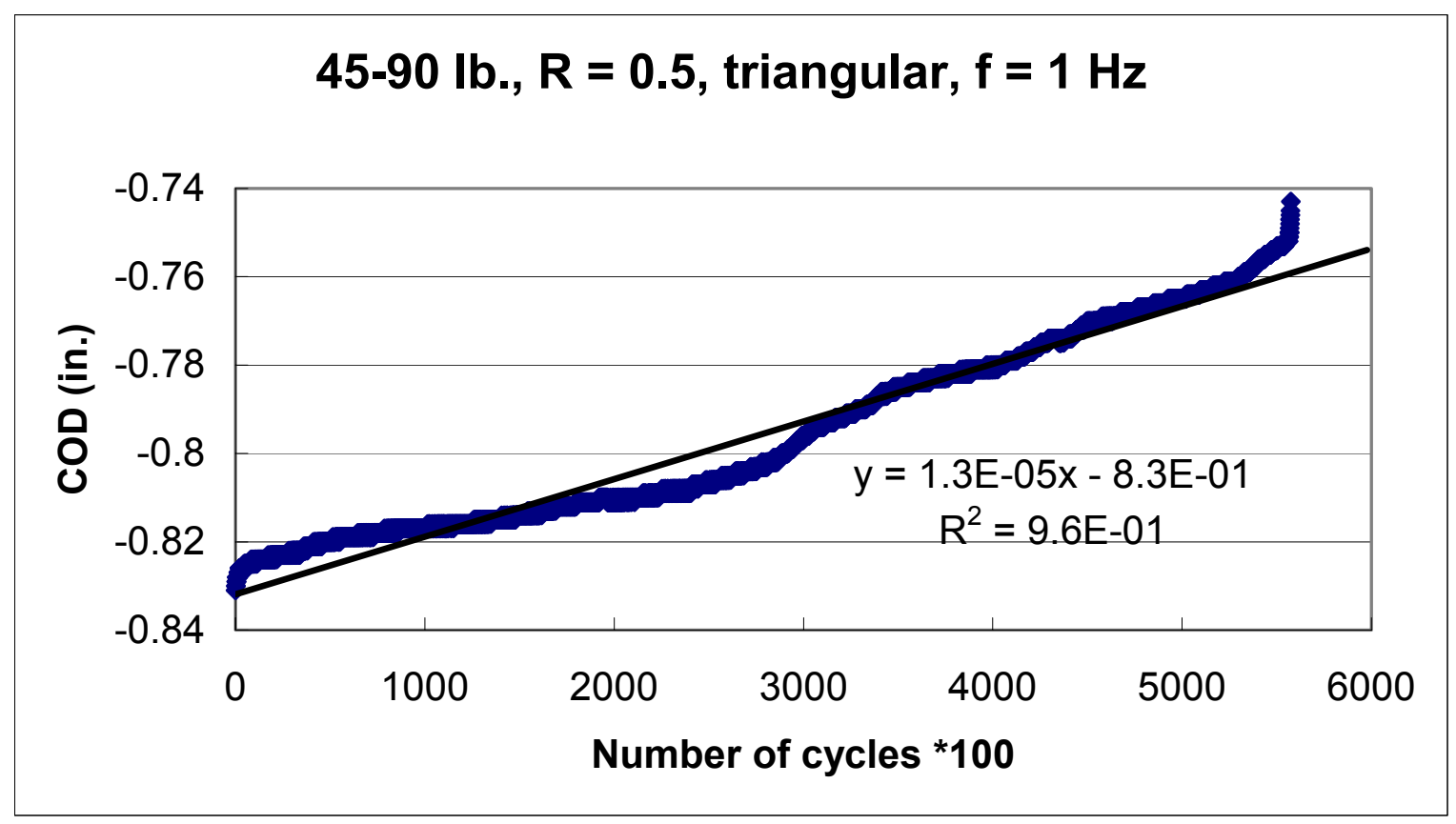

Fig. 4.3 COD versus number of cycles for a CDCB specimen under 45-90 lb. cyclic loading with a frequency $f=1 \mathrm{~Hz}$, load ratio $R=0.5$ and triangular waveform 


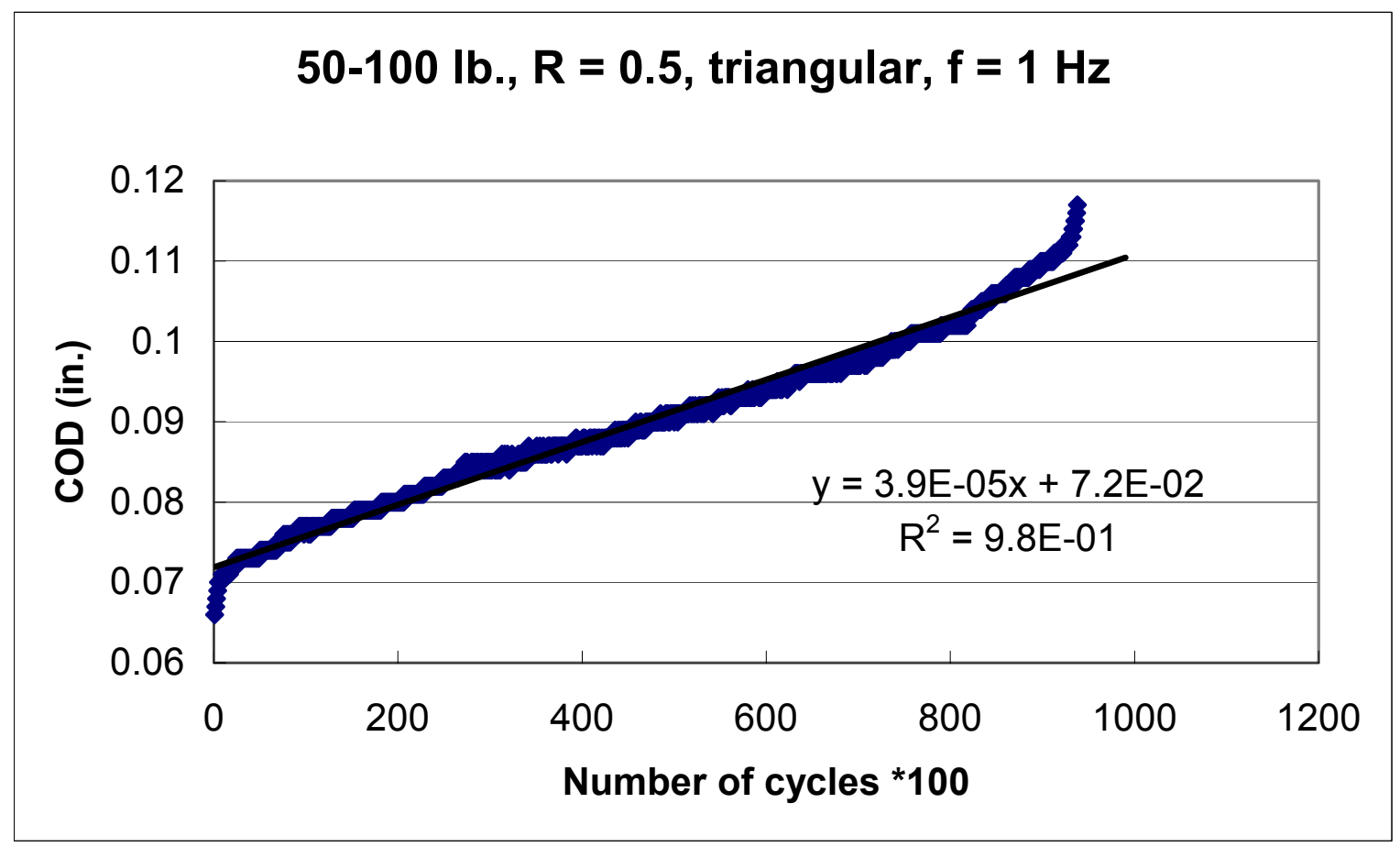

Fig. 4.4 COD versus number of cycles for a CDCB specimen under 50-100 lb. cyclic loading with a frequency $f=1 \mathrm{~Hz}$, load ratio $R=0.5$ and triangular waveform 


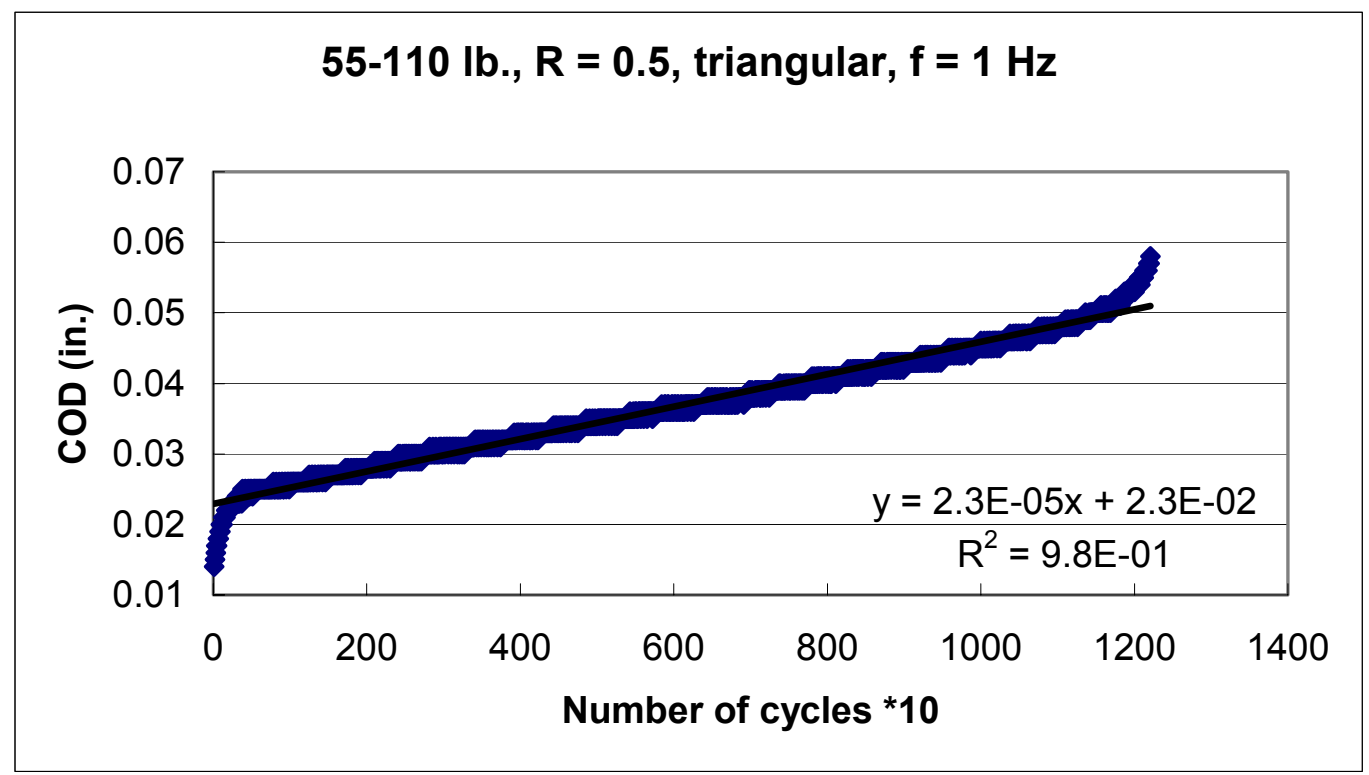

Fig. 4.5 COD versus number of cycles for a CDCB specimen under 55-110 lb. cyclic loading with a frequency $f=1 \mathrm{~Hz}$, load ratio $R=0.5$ and triangular waveform 


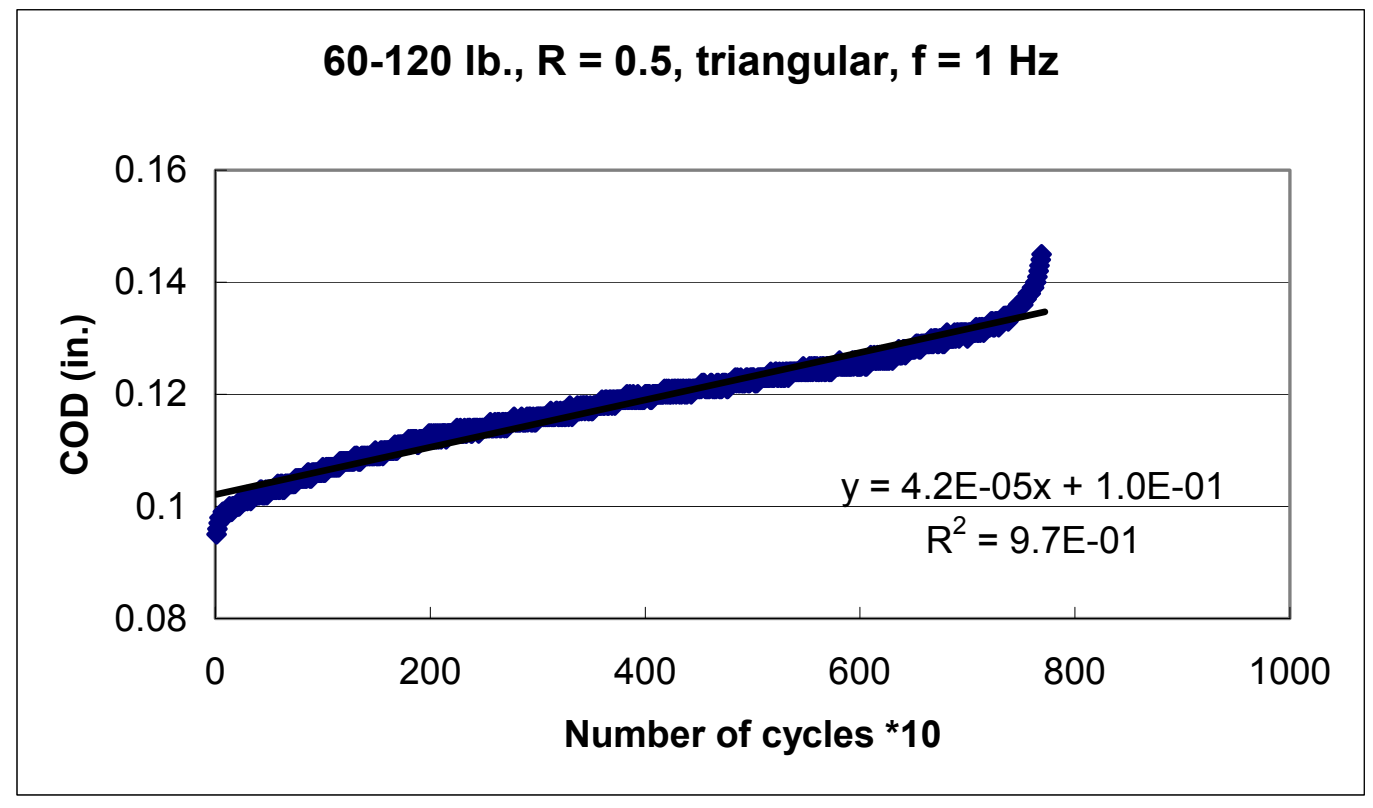

Fig. 4.6 COD versus number of cycles for a CDCB specimen under 60-120 lb. cyclic loading with a frequency $f=1 \mathrm{~Hz}$, load ratio $R=0.5$ and triangular waveform 


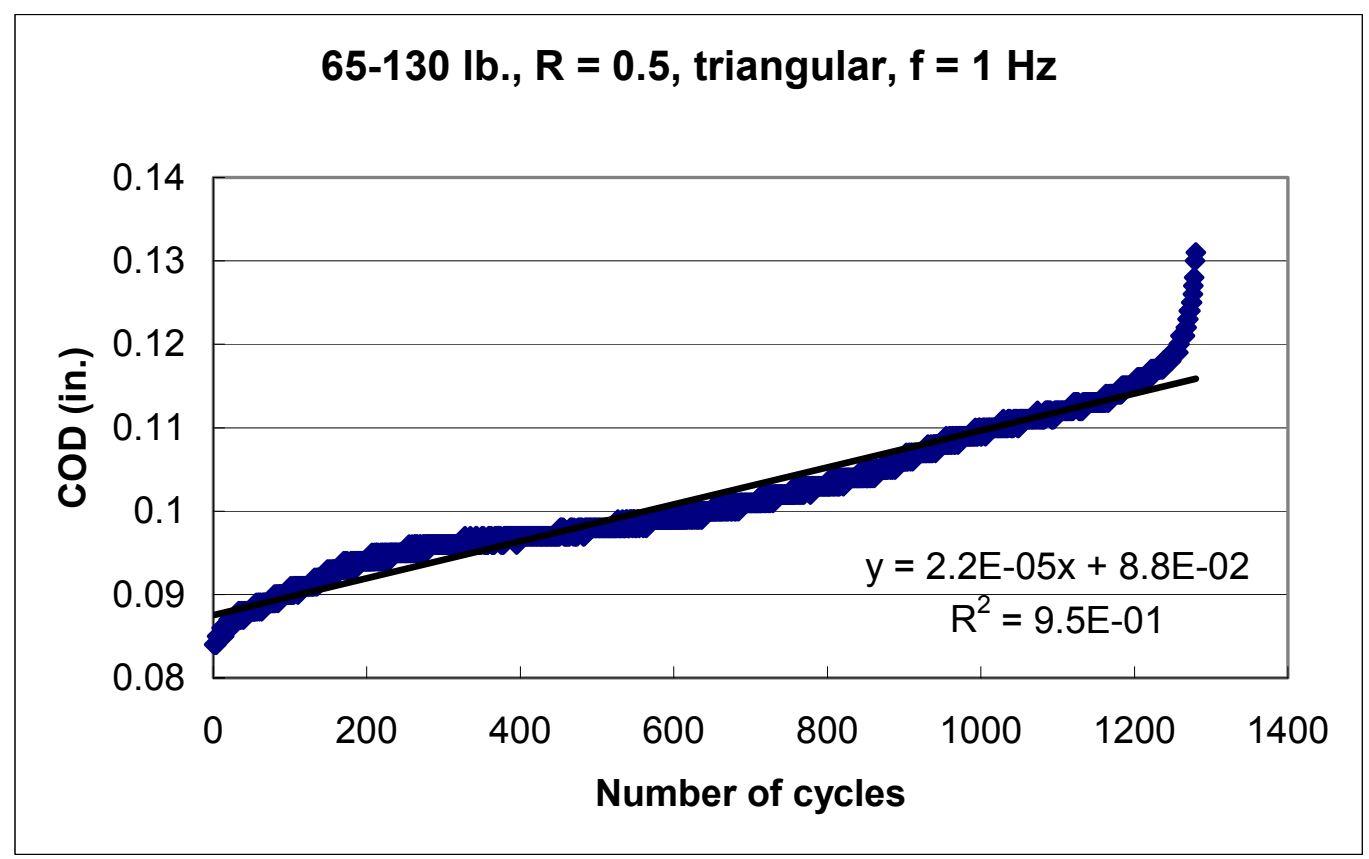

Fig. 4.7 COD versus number of cycles for a CDCB specimen under 65-130 lb. cyclic loading with a frequency $f=1 \mathrm{~Hz}$, load ratio $R=0.5$ and triangular waveform 


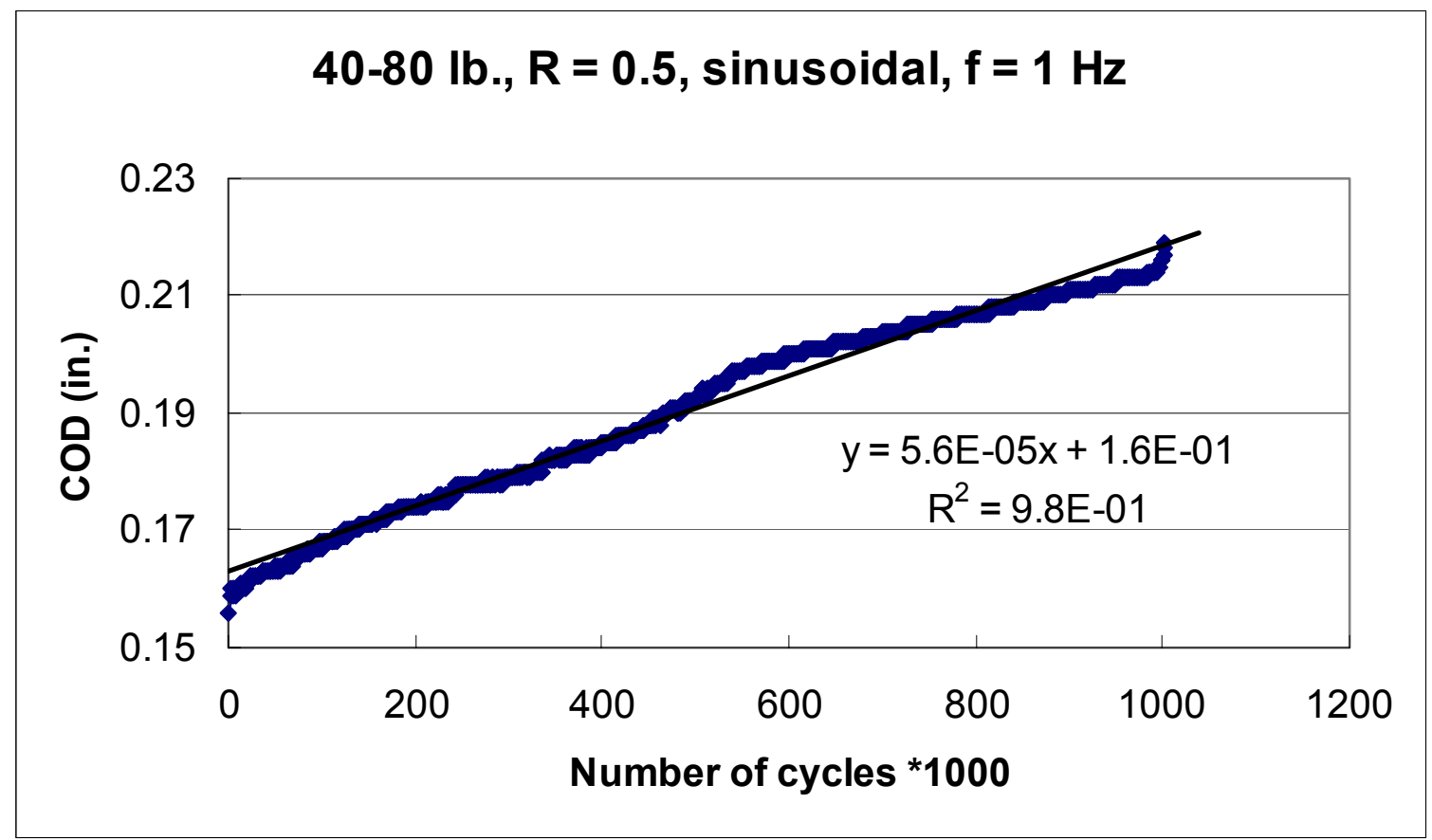

Fig. 4.8 COD versus number of cycles for a CDCB specimen under 40-80 lb. cyclic loading with a frequency $f=1 \mathrm{~Hz}$, load ratio $R=0.5$ and sinusoidal waveform 


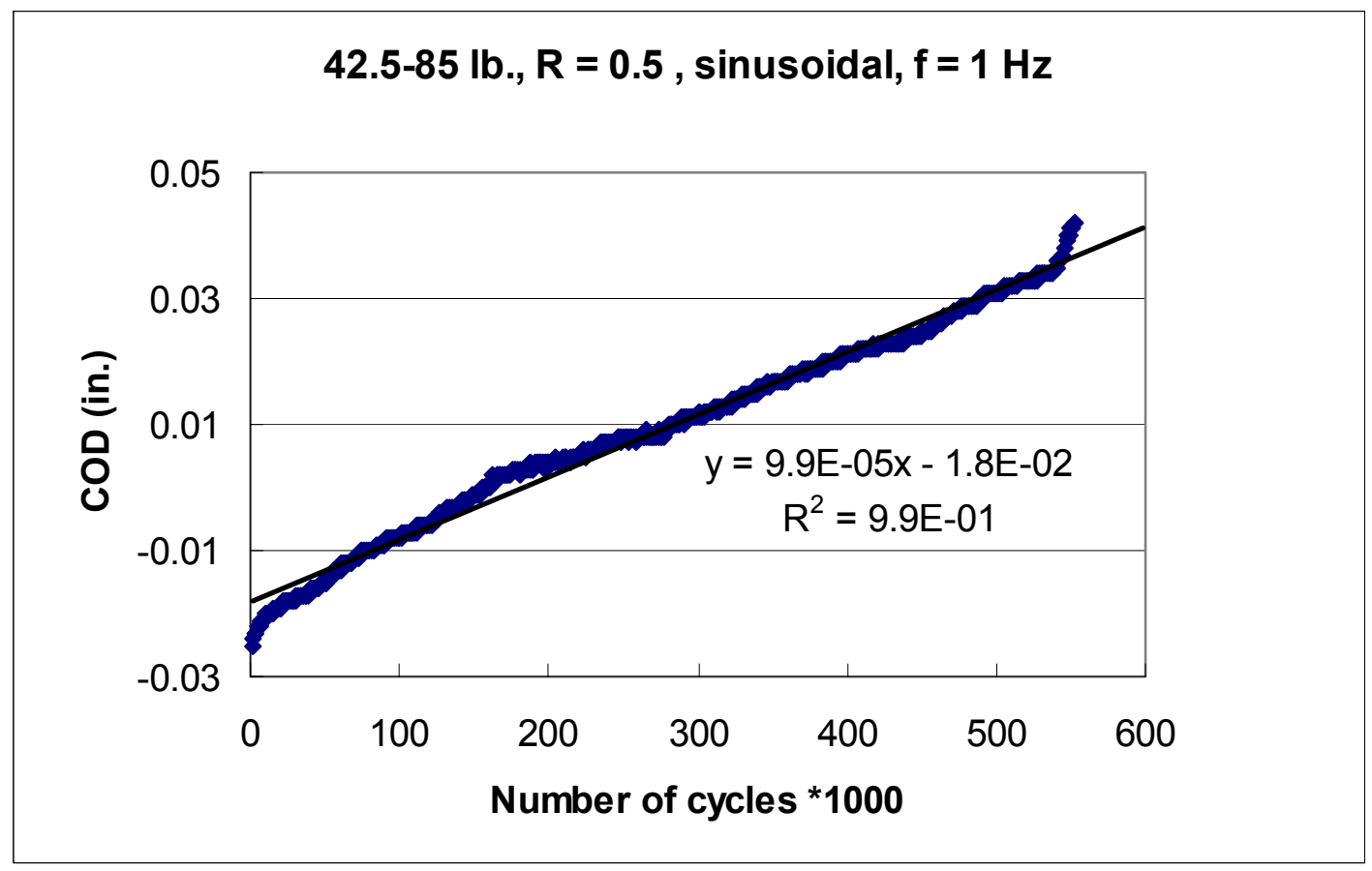

Fig. 4.9 COD versus number of cycles for a CDCB specimen under 42.5-85 lb. cyclic loading with a frequency $f=1 \mathrm{~Hz}$, load ratio $R=0.5$ and sinusoidal waveform 


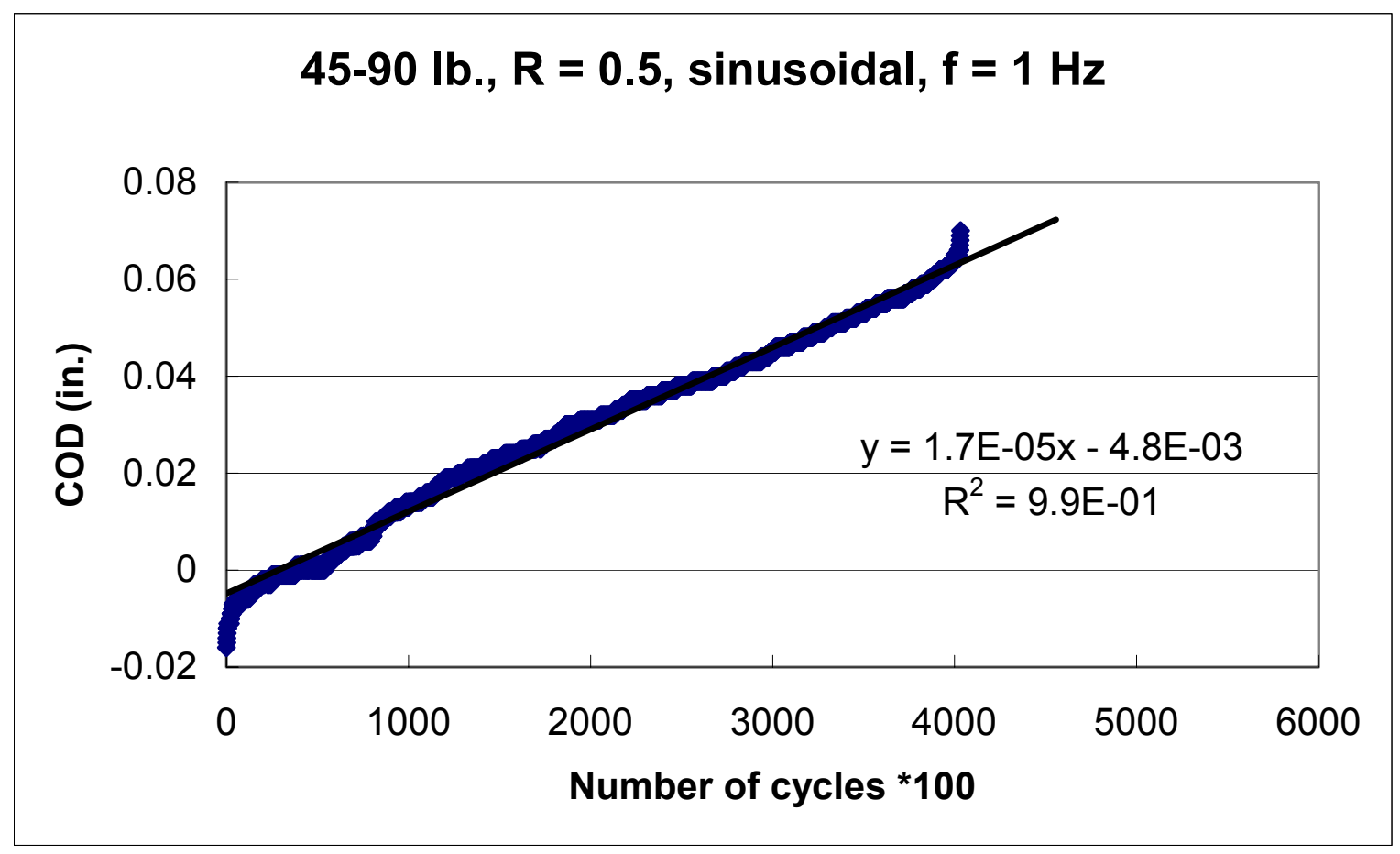

Fig. 4.10 COD versus number of cycles for a CDCB specimen under 45-90 lb. cyclic loading with a frequency $f=1 \mathrm{~Hz}$, load ratio $R=0.5$ and sinusoidal waveform 


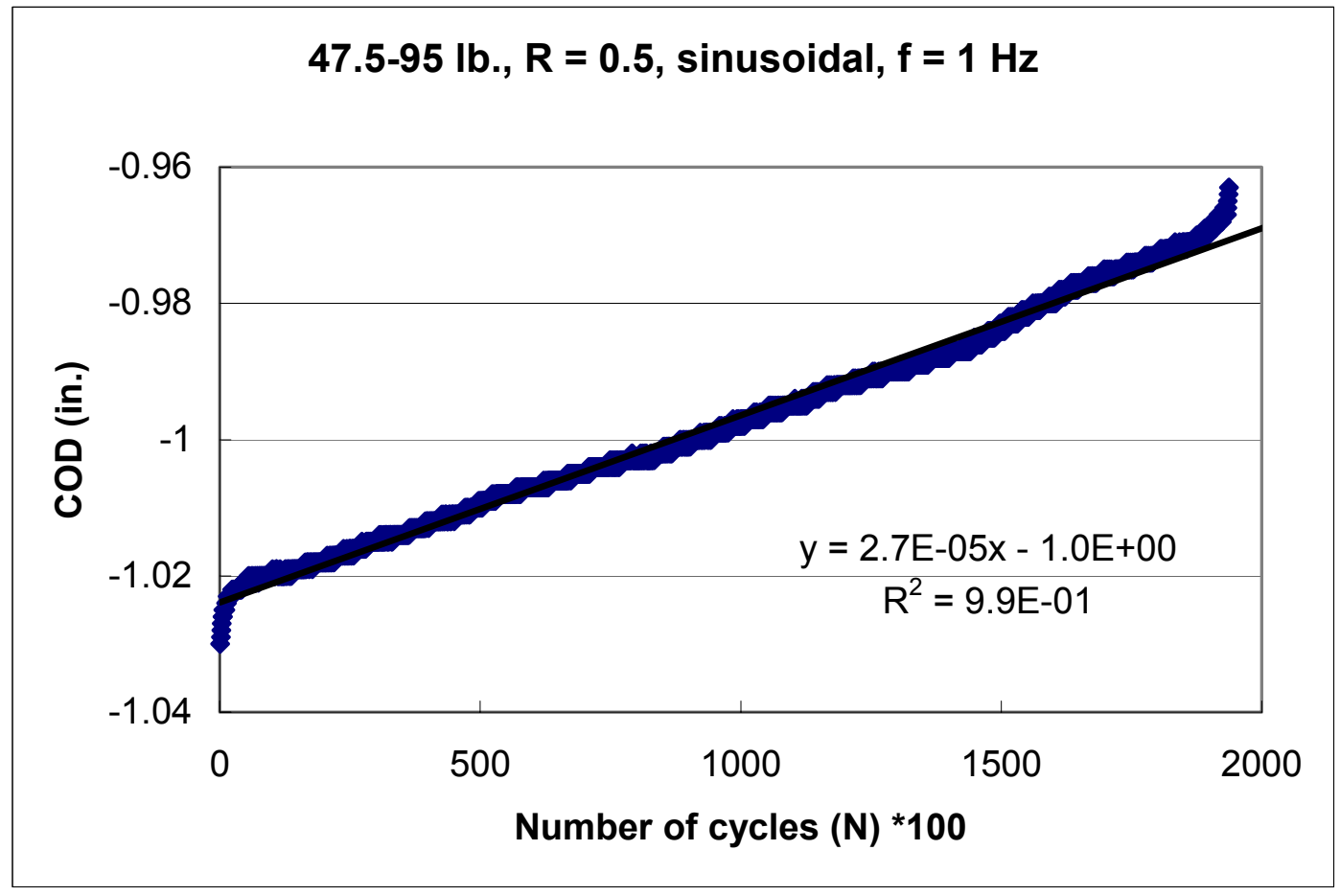

Fig. 4.11 COD versus number of cycles for a CDCB specimen under 47.5-95 lb. cyclic loading with a frequency $f=1 \mathrm{~Hz}$, load ratio $R=0.5$ and sinusoidal waveform 


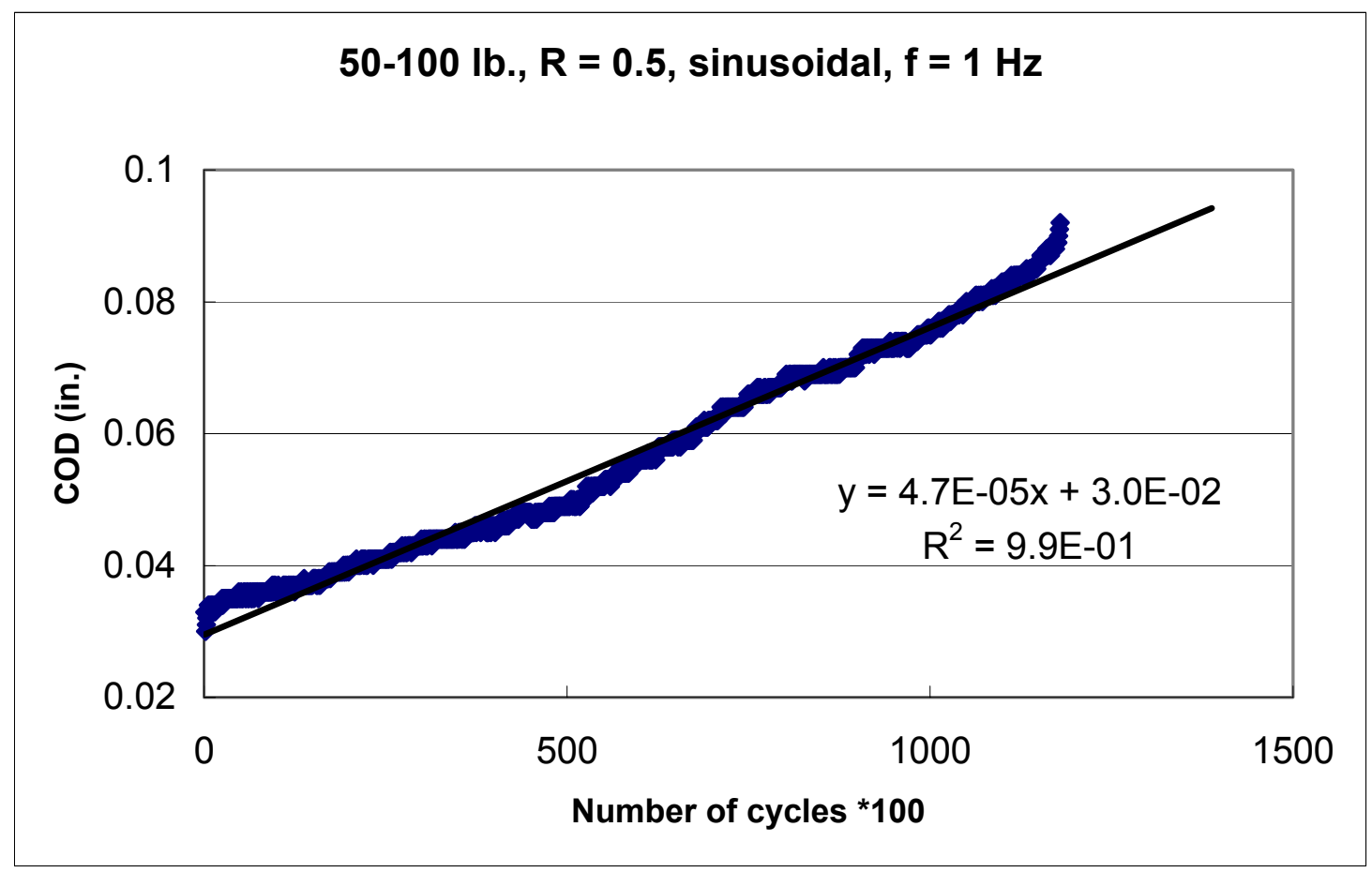

Fig. 4.12 COD versus number of cycles for a CDCB specimen under 50-100 lb. cyclic loading with a frequency $f=1 \mathrm{~Hz}$, load ratio $R=0.5$ and sinusoidal waveform 


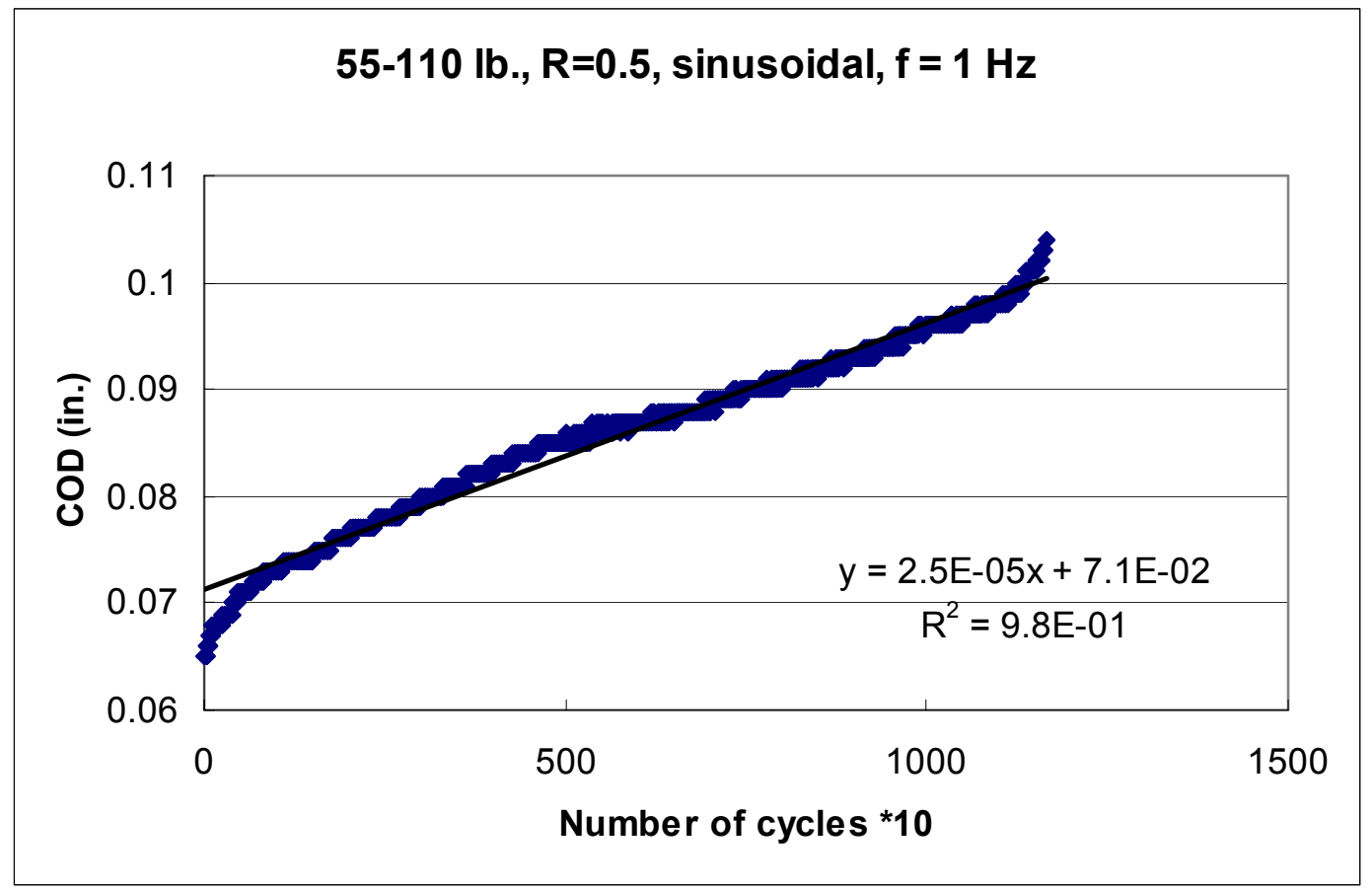

Fig. 4.13 COD versus number of cycles for a CDCB specimen under 55-110 lb. cyclic loading with a frequency $f=1 \mathrm{~Hz}$, load ratio $R=0.5$ and sinusoidal waveform 


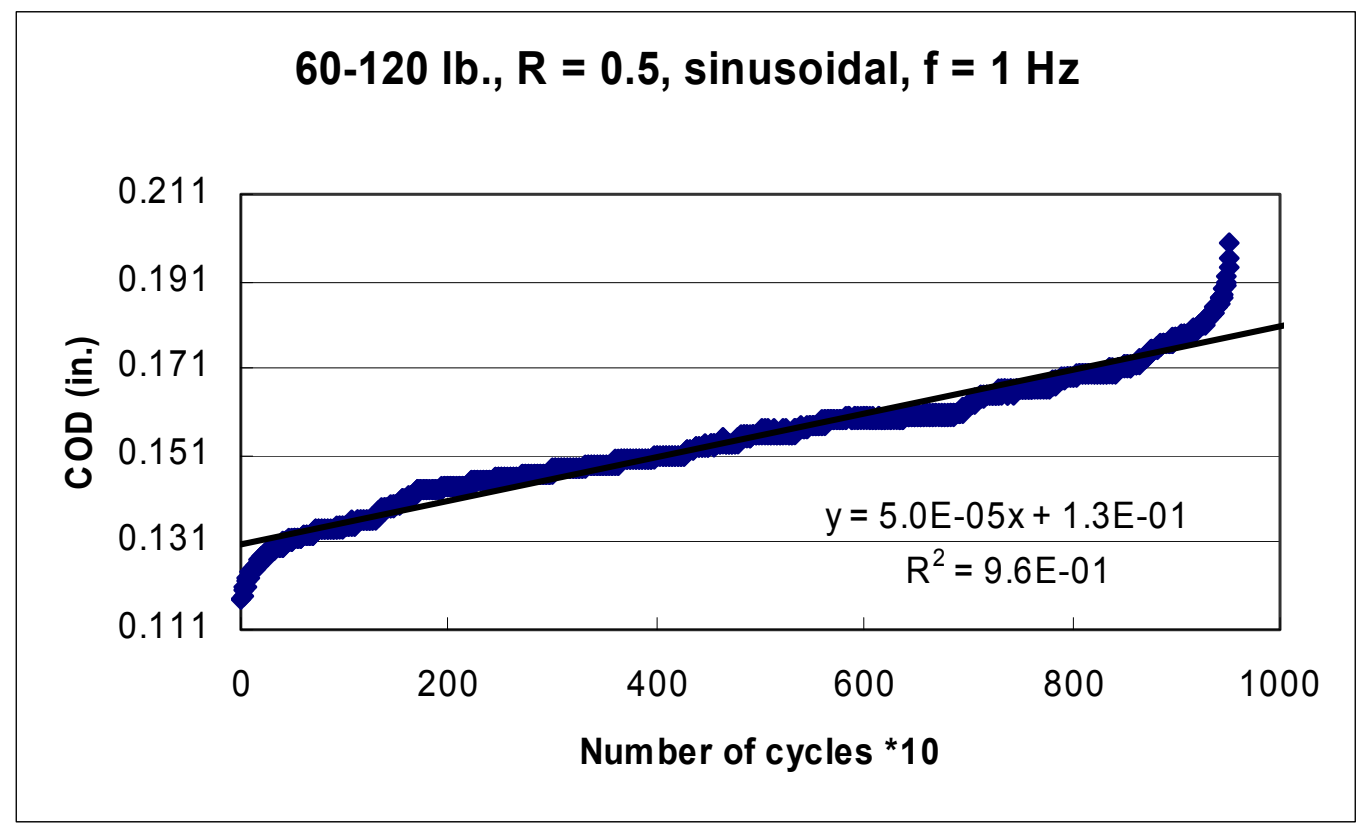

Fig. 4.14 COD versus number of cycles for a CDCB specimen under 60-120 lb. cyclic loading with a frequency $f=1 \mathrm{~Hz}$, load ratio $R=0.5$ and sinusoidal waveform 


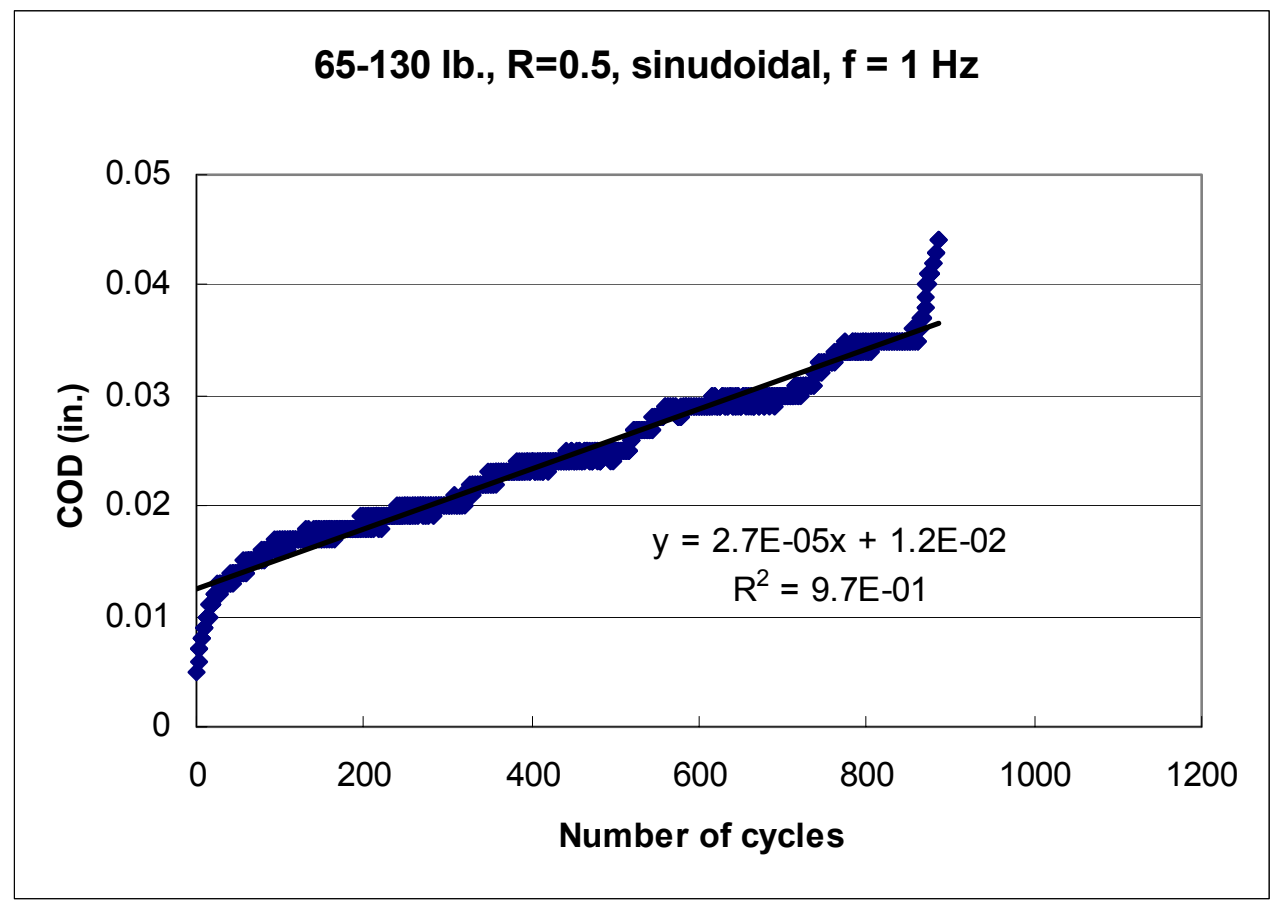

Fig. 4.15 COD versus number of cycles for a CDCB specimen under 65-130 lb. cyclic loading with a frequency $f=1 \mathrm{~Hz}$, load ratio $R=0.5$ and sinusoidal waveform 


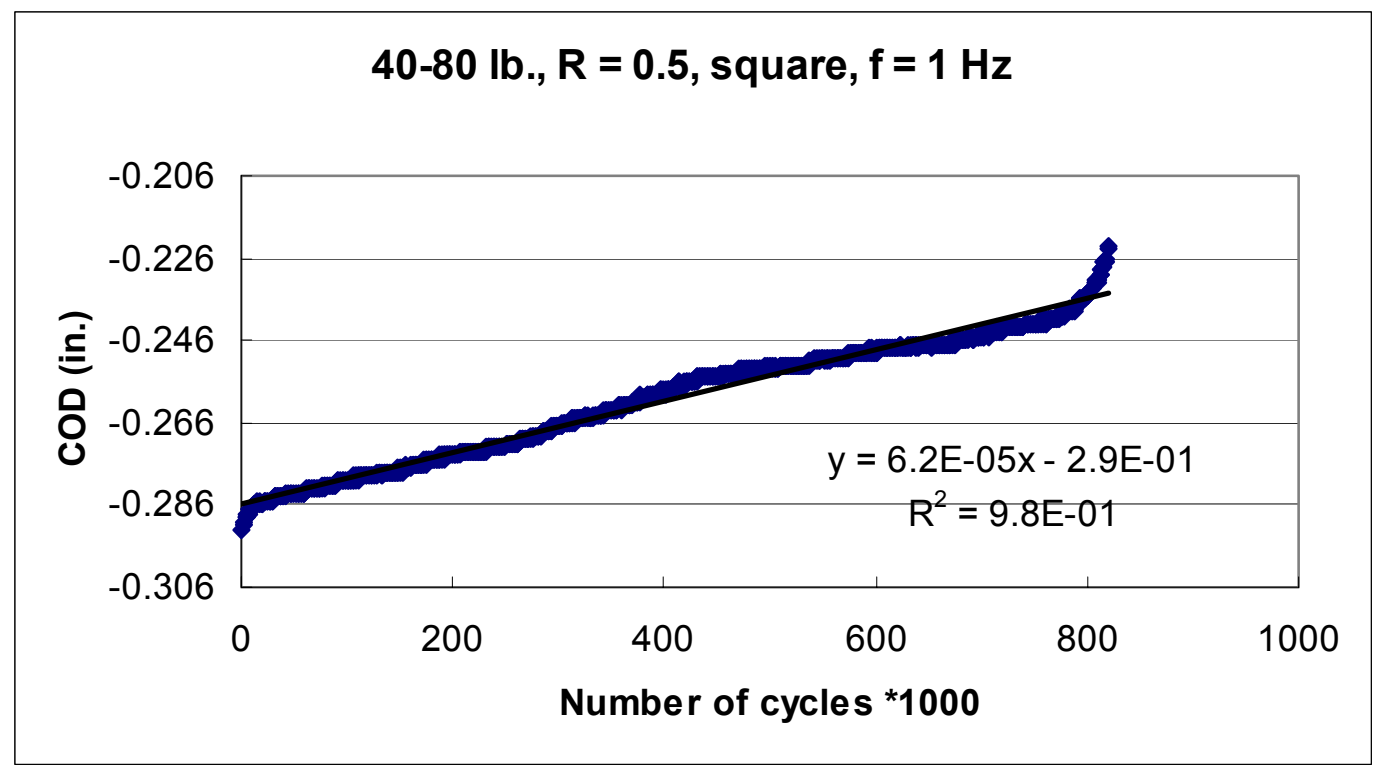

Fig. 4.16 COD versus number of cycles for a CDCB specimen under 40-80 lb. cyclic loading with a frequency $f=1 \mathrm{~Hz}$, load ratio $R=0.5$ and square waveform 


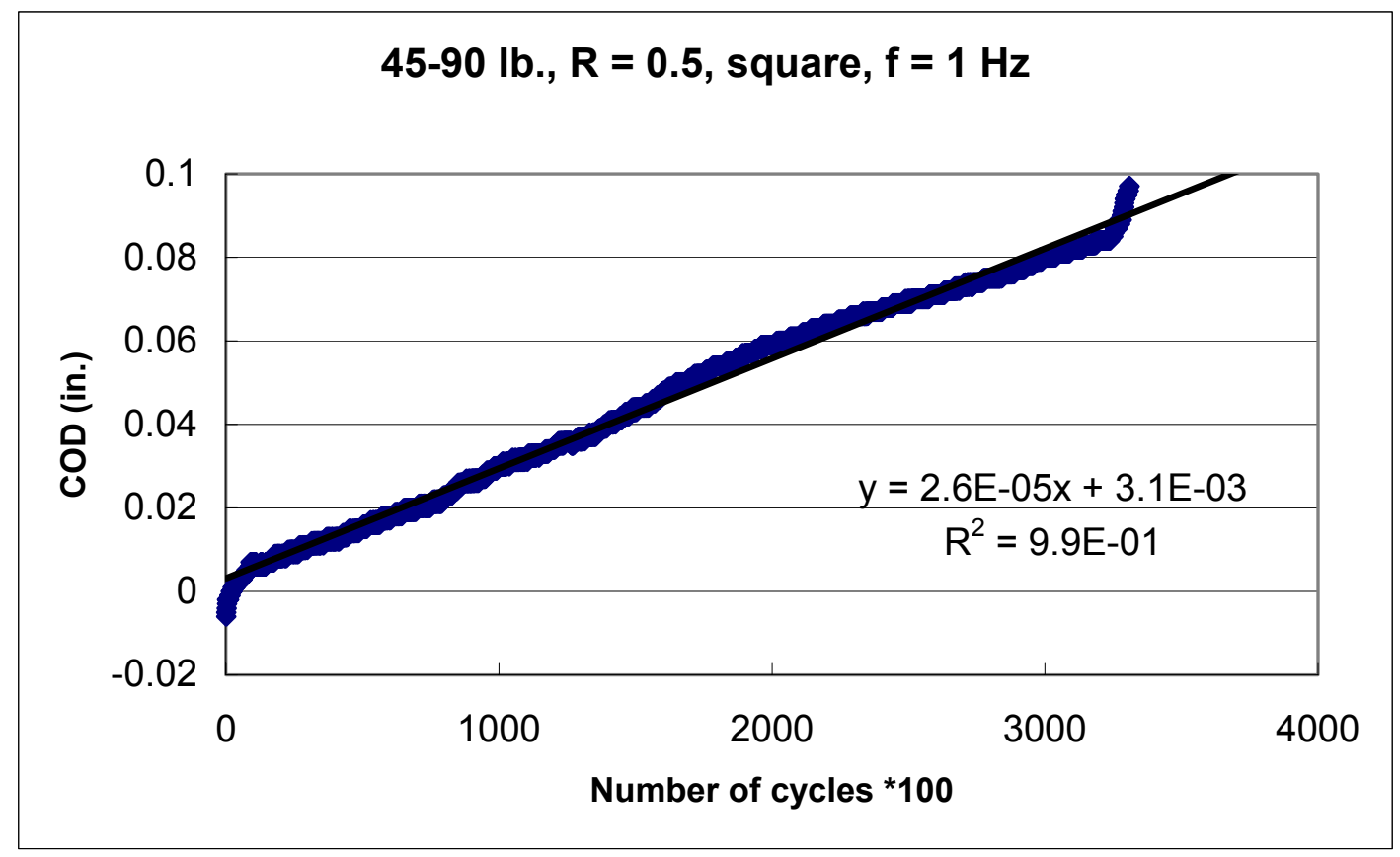

Fig. 4.17 COD versus number of cycles for a CDCB specimen under 45-90 lb. cyclic loading with a frequency $f=1 \mathrm{~Hz}$, load ratio $R=0.5$ and square waveform 


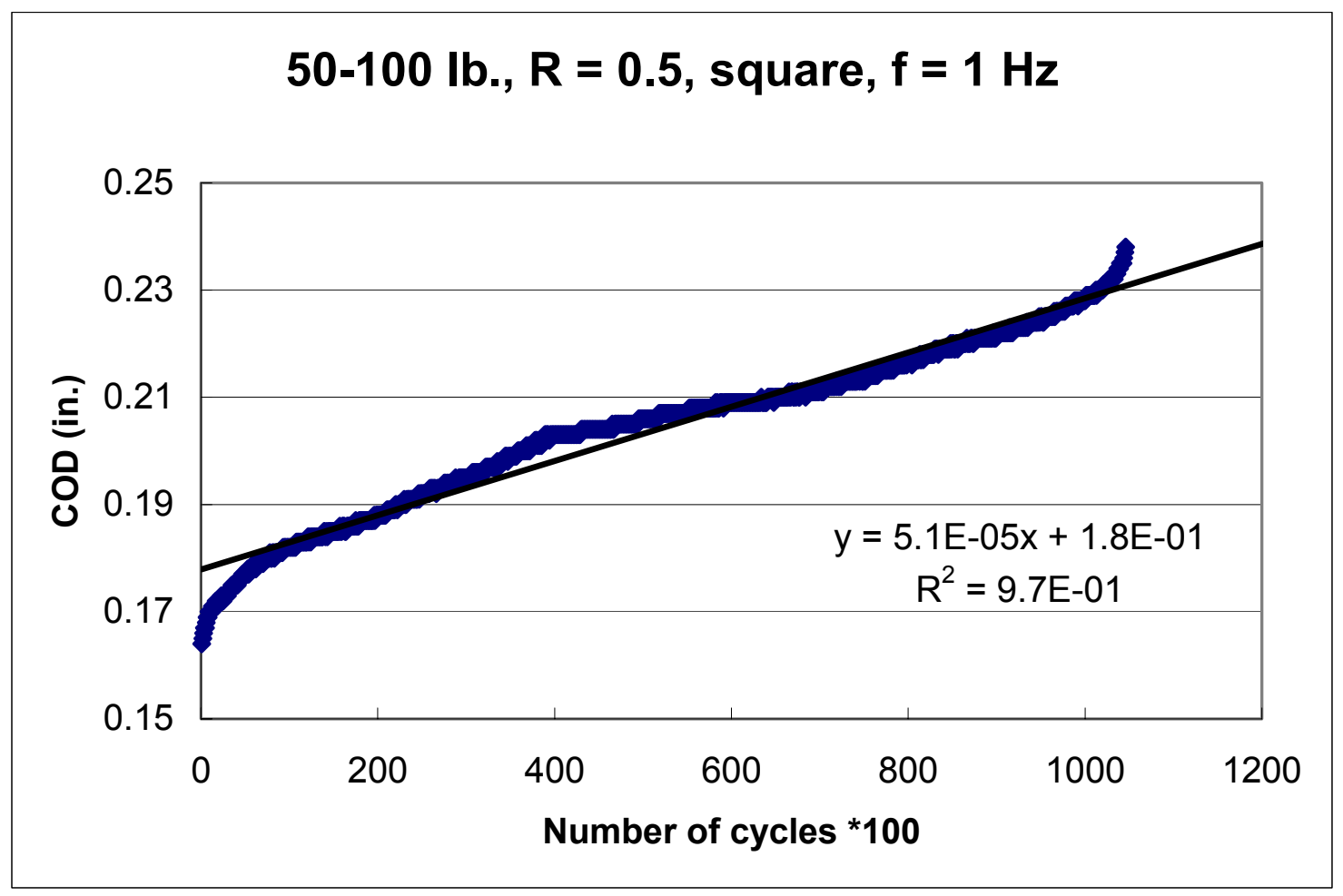

Fig. 4.18 COD versus number of cycles for a CDCB specimen under 50-100 lb. cyclic loading with a frequency $f=1 \mathrm{~Hz}$, load ratio $R=0.5$ and square waveform 


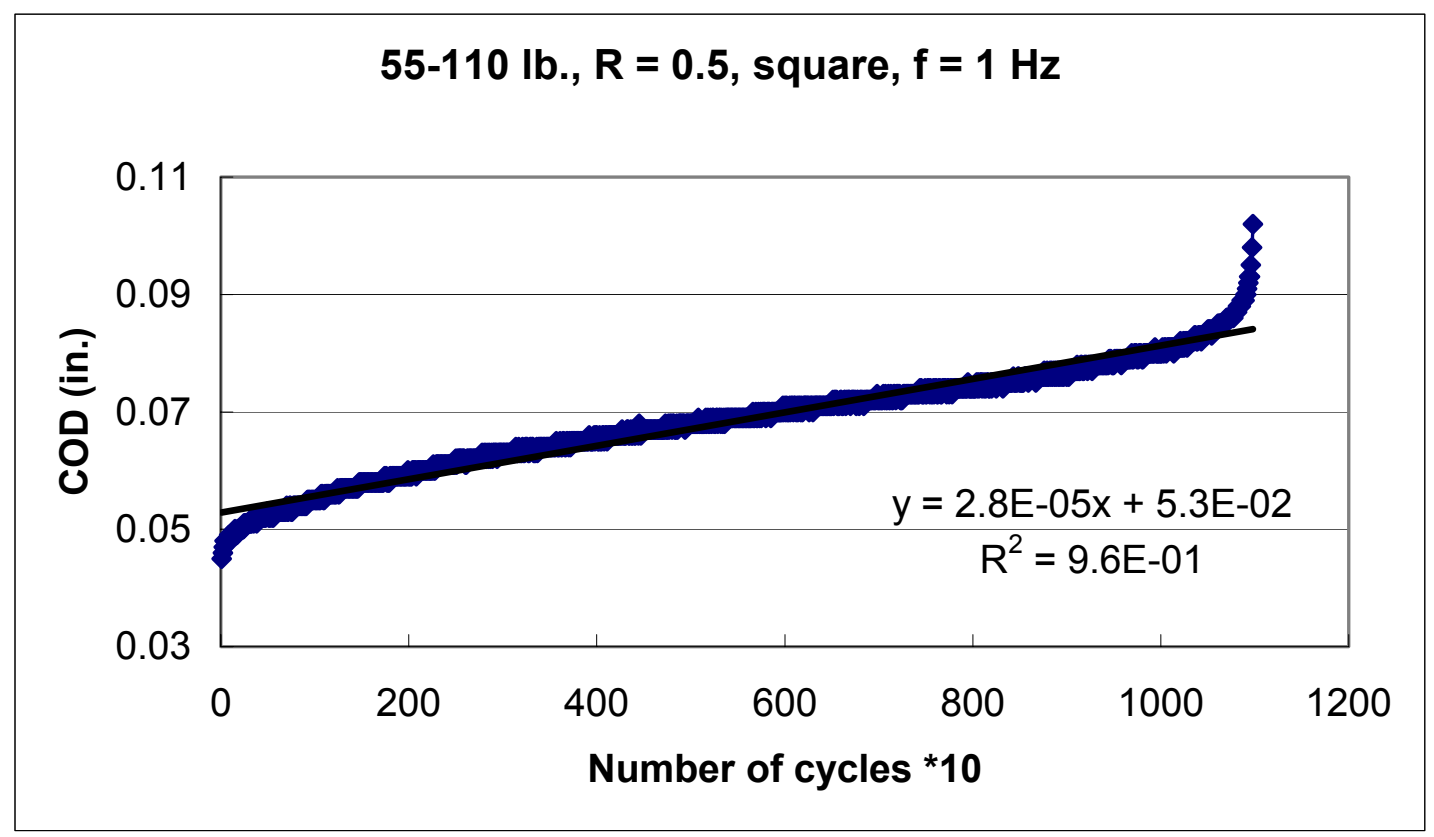

Fig. 4.19 COD versus number of cycles for a CDCB specimen under 55-110 lb. cyclic loading with a frequency $f=1 \mathrm{~Hz}$, load ratio $R=0.5$ and square waveform 


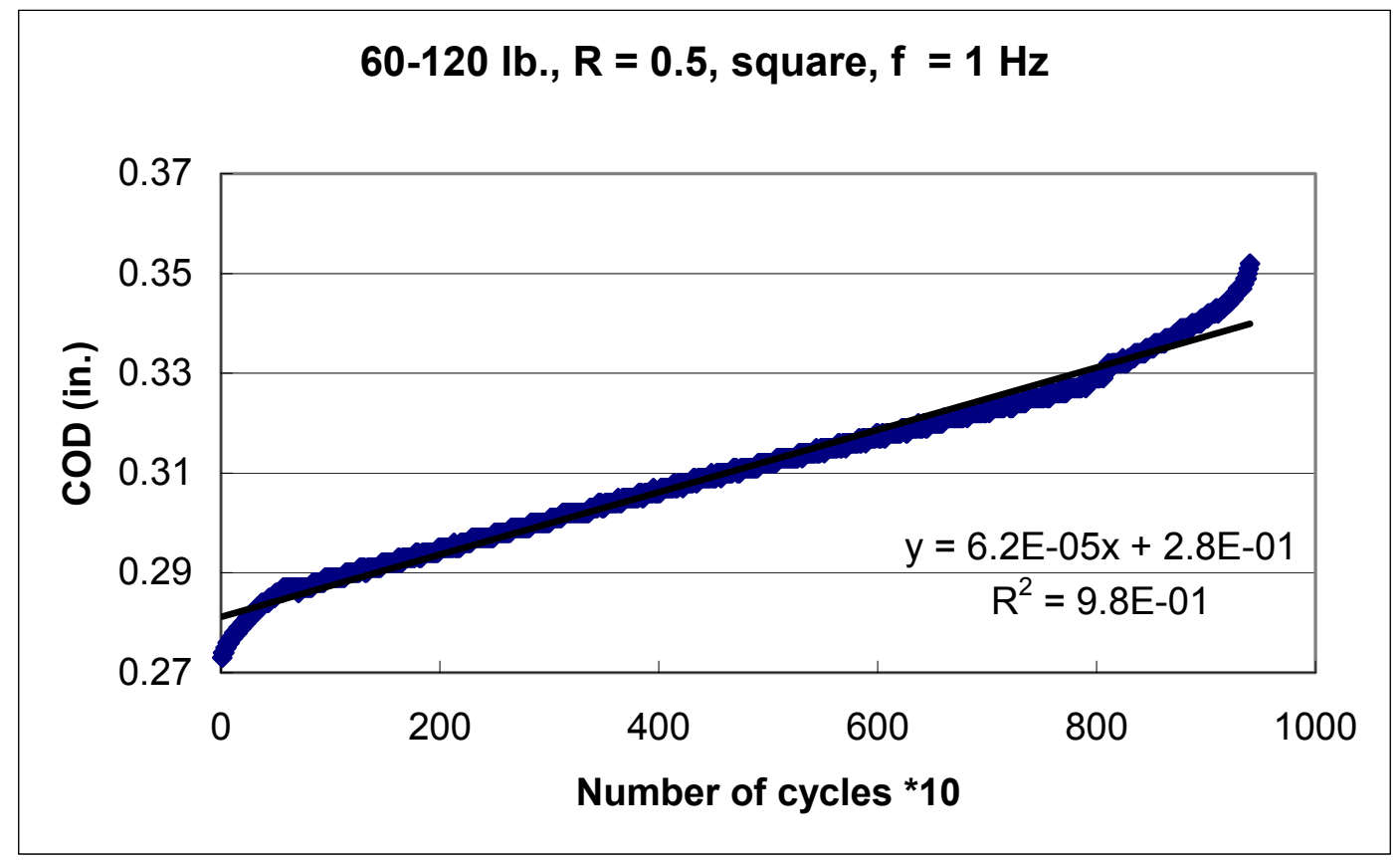

Fig. 4.20 COD versus number of cycles for a CDCB specimen under 60-120 lb. cyclic loading with a frequency $f=1 \mathrm{~Hz}$, load ratio $R=0.5$ and square waveform 


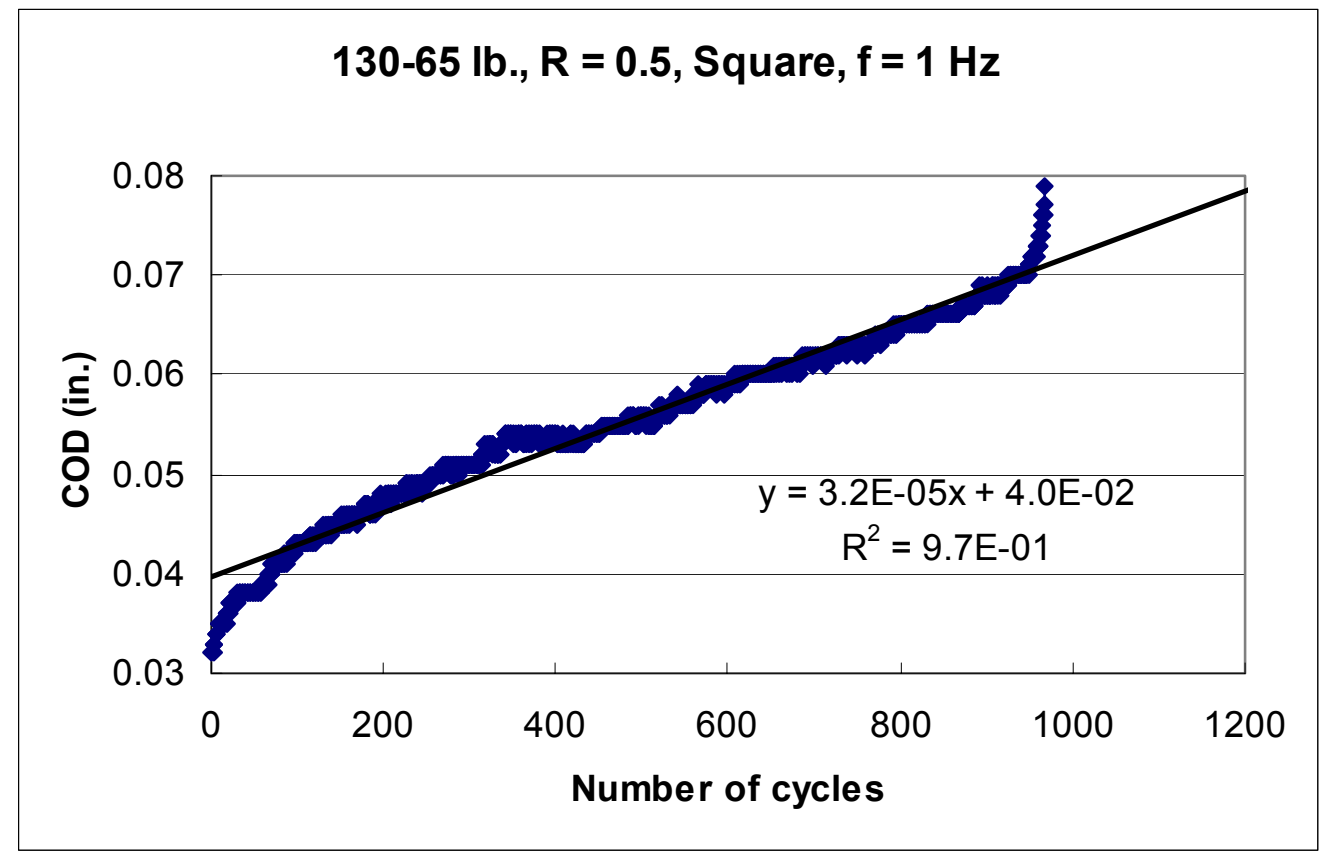

Fig. 4.21 COD versus number of cycles for a CDCB specimen under $65-130 \mathrm{lb}$. cyclic loading with a frequency $f=1 \mathrm{~Hz}$, load ratio $R=0.5$ and square waveform 
Table 4.4 Experimental results of the COD propagation rate for the triangular waveform under the constant load ratio $R=0.5$ and frequency $f=1 \mathrm{~Hz}$

\begin{tabular}{ccc}
\hline$P_{\max }(\mathrm{lb})$. & $P_{\min }(\mathrm{lb})$. & $d C O D / d N($ in./cycle $)$ \\
130 & 65 & $2.20 \mathrm{E}-05$ \\
120 & 60 & $4.20 \mathrm{E}-06$ \\
110 & 55 & $2.30 \mathrm{E}-06$ \\
100 & 50 & $3.90 \mathrm{E}-07$ \\
90 & 45 & $1.30 \mathrm{E}-07$ \\
80 & 40 & $4.40 \mathrm{E}-08$ \\
\hline
\end{tabular}

Table 4.5 Experimental results of the COD propagation rate for the sinusoidal waveform under the constant load ratio $R=0.5$ and frequency $f=1 \mathrm{~Hz}$

\begin{tabular}{ccc}
\hline$P_{\max }(\mathrm{lb})$. & $P_{\min }(\mathrm{lb})$. & $d C O D / d N($ in./cycle $)$ \\
130 & 65 & $2.70 \mathrm{E}-05$ \\
120 & 60 & $5.00 \mathrm{E}-06$ \\
110 & 55 & $2.50 \mathrm{E}-06$ \\
100 & 50 & $4.70 \mathrm{E}-07$ \\
95 & 47.5 & $2.70 \mathrm{E}-07$ \\
90 & 45 & $1.70 \mathrm{E}-07$ \\
85 & 42.5 & $9.90 \mathrm{E}-08$ \\
80 & 40 & $5.60 \mathrm{E}-08$ \\
\hline
\end{tabular}


Table 4.6 Experimental results of the COD propagation rate for the square waveform under the constant load ratio $R=0.5$ and frequency $f=1 \mathrm{~Hz}$

\begin{tabular}{ccc}
\hline$P_{\max }(\mathrm{lb})$. & $P_{\min }(\mathrm{lb})$. & $d C O D / d N($ in./cycle $)$ \\
130 & 65 & $3.20 \mathrm{E}-05$ \\
120 & 60 & $6.20 \mathrm{E}-06$ \\
110 & 55 & $2.80 \mathrm{E}-06$ \\
100 & 50 & $5.10 \mathrm{E}-07$ \\
90 & 45 & $2.60 \mathrm{E}-07$ \\
80 & 40 & $6.20 \mathrm{E}-08$ \\
\hline
\end{tabular}

\subsubsection{Crack growth rate determination by compliance method}

The compliance method is used to determine the crack growth rate. In general, measuring crack length is a very difficult task. Fatigue tests could last several weeks. This makes the measurement of the crack length more difficult. On the other hand, the crack opening displacement (COD) is much easier to measure than the crack length for the CDCB specimen. The difficulty of measuring crack propagation rate can be avoided by recording COD propagation rate, which is easily obtained by the data acquisition 
system. Again, the transformation from crack opening displacement propagation rate $d C O D / d N$ to crack propagation rate $d a / d N$ is derived by using the chain rule and given as

$$
\frac{d a}{d N}=\frac{d a}{d C} \frac{d C}{d N}=\frac{1}{k P} \frac{d C O D}{d N}
$$

where $N$ is the number of cycles, $P$ is the applied load, and $k$ is a constant that is equal to the $d C / d a$ value of the CDCB specimen.

\subsubsection{Constant strain energy release rate range calculation}

The contoured double cantilever beam (CDCB) specimen was used for evaluating fracture toughness of bonded wood-FRP interfaces under mode-I loading. The strain energy release rate $G$ is given as

$$
G=\frac{P^{2}}{2 b} \frac{d C}{d a}
$$

where, $P=$ applied load; $b=$ thickness of the specimen; $C=$ compliance; and $a=$ crack length. For one complete cycle, the maximum and minimum strain energy release rates are given

$$
G_{\max }=\frac{P_{\max }^{2}}{2 b} \frac{d C}{d a}
$$




$$
G_{\min }=\frac{P_{\min }^{2}}{2 b} \frac{d C}{d a}
$$

The strain energy release rate range $\Delta G$ is easily obtained from the difference of equations (4.4) and (4.5). For the CDCB specimen, the rate of change of compliance with respect to crack length $d C / d a$ is constant, and therefore, the constant strain energy release rate range $\Delta G$ is acquired for constant cyclic loading. The crack propagation rate $d a / d N$ and strain energy release rate parameters are obtained by using Eqs. 4.2, 4.4 and 4.5, and are listed in Tables 4.7, 4.8 and 4.9.

Table 4.7 The crack propagation rate $d a / d N$ for triangular waveform

\begin{tabular}{ccccc}
\hline$G_{\max }$ (lb./in.) & $G_{\min }(\mathrm{lb} . /$ in. $)$ & $\Delta G$ (lb./in.) & $d C O D / d N($ in./cycle $)$ & $d a / d N($ in./cycle $)$ \\
\hline 2.00 & 0.50 & 1.50 & $2.20 \mathrm{E}-05$ & $5.72 \mathrm{E}-04$ \\
1.70 & 0.43 & 1.28 & $4.20 \mathrm{E}-06$ & $1.18 \mathrm{E}-04$ \\
1.43 & 0.36 & 1.07 & $2.30 \mathrm{E}-06$ & $7.07 \mathrm{E}-05$ \\
1.18 & 0.30 & 0.89 & $3.90 \mathrm{E}-07$ & $1.32 \mathrm{E}-05$ \\
0.96 & 0.24 & 0.72 & $1.30 \mathrm{E}-07$ & $4.88 \mathrm{E}-06$ \\
0.76 & 0.19 & 0.57 & $4.40 \mathrm{E}-08$ & $1.86 \mathrm{E}-06$ \\
\hline
\end{tabular}


Table 4.8 The crack propagation rate $d a / d N$ for sinudoidal waveform

\begin{tabular}{ccccc}
\hline$G_{\max }($ lb./in.) & $G_{\min }(\mathrm{lb} . /$ in. $)$ & $\Delta G(\mathrm{lb} . / \mathrm{in})$. & $d C O D / d N($ in./cycle $)$ & $d a / d N($ in./cycle $)$ \\
\hline 2.00 & 0.50 & 1.50 & $2.70 \mathrm{E}-05$ & $7.02 \mathrm{E}-04$ \\
1.70 & 0.43 & 1.28 & $5.00 \mathrm{E}-06$ & $1.41 \mathrm{E}-04$ \\
1.43 & 0.36 & 1.07 & $2.50 \mathrm{E}-06$ & $7.68 \mathrm{E}-05$ \\
1.18 & 0.30 & 0.89 & $4.70 \mathrm{E}-07$ & $1.59 \mathrm{E}-05$ \\
1.07 & 0.27 & 0.80 & $2.70 \mathrm{E}-07$ & $9.60 \mathrm{E}-06$ \\
0.96 & 0.24 & 0.72 & $1.70 \mathrm{E}-07$ & $6.38 \mathrm{E}-06$ \\
0.86 & 0.21 & 0.64 & $9.90 \mathrm{E}-08$ & $3.94 \mathrm{E}-06$ \\
0.76 & 0.19 & 0.57 & $5.60 \mathrm{E}-08$ & $2.37 \mathrm{E}-06$ \\
\hline
\end{tabular}

Table 4.9 The crack propagation rate $d a / d N$ for square waveform

\begin{tabular}{ccccc}
\hline$G_{\max }(\mathrm{lb} . / \mathrm{in})$. & $G_{\min }(\mathrm{lb} . /$ in. $)$ & $\Delta G(\mathrm{lb} . / \mathrm{in})$. & $d C O D / d N($ in./cycle $)$ & $d a / d N($ in./cycle $)$ \\
\hline 2.00 & 0.50 & 1.50 & $3.20 \mathrm{E}-05$ & $8.32 \mathrm{E}-04$ \\
1.70 & 0.43 & 1.28 & $6.20 \mathrm{E}-06$ & $1.75 \mathrm{E}-04$ \\
1.43 & 0.36 & 1.07 & $2.80 \mathrm{E}-06$ & $8.60 \mathrm{E}-05$ \\
1.18 & 0.30 & 0.89 & $5.10 \mathrm{E}-07$ & $1.72 \mathrm{E}-05$ \\
0.96 & 0.24 & 0.72 & $2.60 \mathrm{E}-07$ & $9.76 \mathrm{E}-06$ \\
0.76 & 0.19 & 0.57 & $6.20 \mathrm{E}-08$ & $2.62 \mathrm{E}-06$ \\
\hline
\end{tabular}




\subsubsection{Waveform effect}

Again, the Paris Law equation corresponding to the strain energy release rate

$$
\frac{d a}{d N}=B(\Delta G)^{m}
$$

is used to fit the data points in Tables 4.7, 4.8 and 4.9. The relationship of crack propagation rate $d a / d N$ versus the strain energy release rate range $\Delta G$ is shown in Figs. 4.22, 4.23 and 4.24. For each wave shape, the constants $B$ and $m$ are listed in Table 4.10. The constant $B$ increases and $m$ decreases from triangular to sinusoidal to square waveforms. However, the differences of $B$ and $m$ among these three different wave shapes are very small, especially for the power coefficient the biggest difference is less than 3 percent. Two more data points are obtained for triangular and square waveforms from Eqs. (4.6) and are listed in Tables 4.11 and 4.12.

Table 4.10 Material constants $B$ and $m$ in Eq. (4.6) for three different waveforms under a constant load ratio $R=0.5$ and a frequency $f=1 \mathrm{~Hz}$

\begin{tabular}{|c|c|c|}
\hline Waveforms & $B$ & $m$ \\
\hline Triangular & $4 \times 10^{-5}$ & 5.85 \\
\hline Sinusoidal & $5 \times 10^{-5}$ & 5.76 \\
\hline Square & $6 \times 10^{-5}$ & 5.72 \\
\hline
\end{tabular}


Table 4.11 The crack propagation rate $d a / d N$ for triangular waveform

\begin{tabular}{cccc}
\hline$G_{\max }(\mathrm{lb} . / \mathrm{in})$. & $G_{\min }(\mathrm{lb} . /$ in. $)$ & $\Delta G(\mathrm{lb} . / \mathrm{in})$. & $d a / d N($ in./cycle $)$ \\
\hline 1.07 & 0.27 & 0.80 & $1.09 \mathrm{E}-05$ \\
0.86 & 0.21 & 0.64 & $2.98 \mathrm{E}-06$ \\
\hline
\end{tabular}

Table 4.12 The crack propagation rate $d a / d N$ for square waveform

\begin{tabular}{cccc}
\hline$G_{\max }(\mathrm{lb} . / \mathrm{in})$. & $G_{\min }(\mathrm{lb} . / \mathrm{in})$. & $\Delta G(\mathrm{lb} . / \mathrm{in})$. & $d a / d N($ in./cycle $)$ \\
\hline 1.07 & 0.27 & 0.80 & $1.69 \mathrm{E}-05$ \\
0.86 & 0.21 & 0.64 & $4.72 \mathrm{E}-06$ \\
\hline
\end{tabular}

As noticed by most researchers, waveform has little or negligible effect on fatigue behavior of various materials. The double log plot of crack propagation rate $d a / d N$ versus the strain energy release rate range $\Delta G$ for different waveforms is presented in Fig. 4.25. For a given $\Delta G$ value, the crack propagation rate $d a / d N$ value for square waveform is a little bit higher than for triangular and sinusoidal waveforms, for which the values nearly coincide. The reason may be that the waveforms for triangular and sinusoidal shapes are very similar, while the square waveform splits into two halves for minimum and maximum loads. However, the difference between the trend lines of $d a / d N$ versus $\Delta G$ for square, triangular and sinusoidal waveforms is not noticeable. Thus, we conclude that the effect of waveforms considered here for the present interface bond can be neglected. 


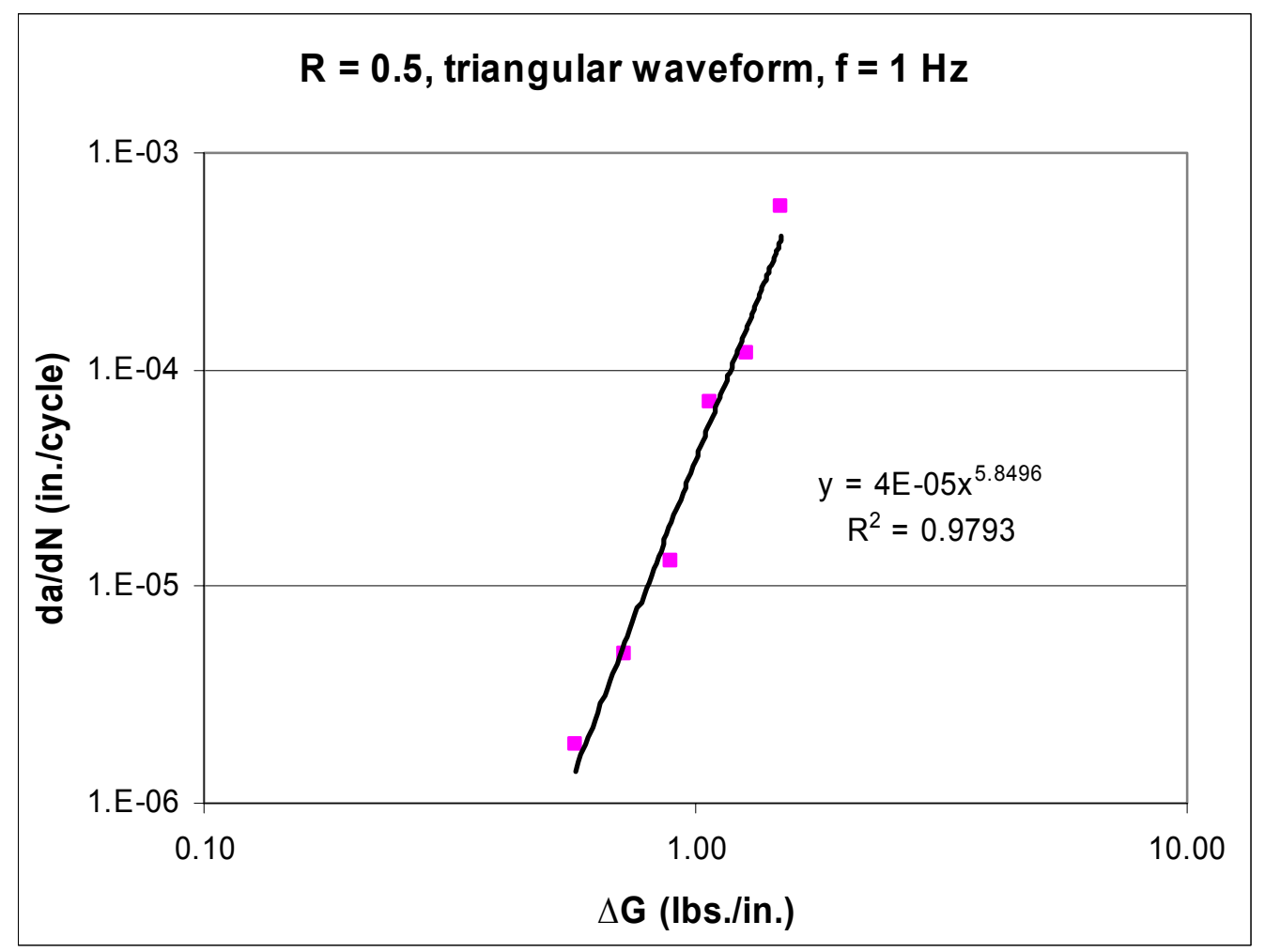

Fig. 4.22 The log-log plot of the crack propagation rate $d a / d N$ versus the strain energy release rate range $\Delta G$ for the triangular waveform under a constant load ratio $R=0.5$ and a frequency $f=1 \mathrm{~Hz}$ 


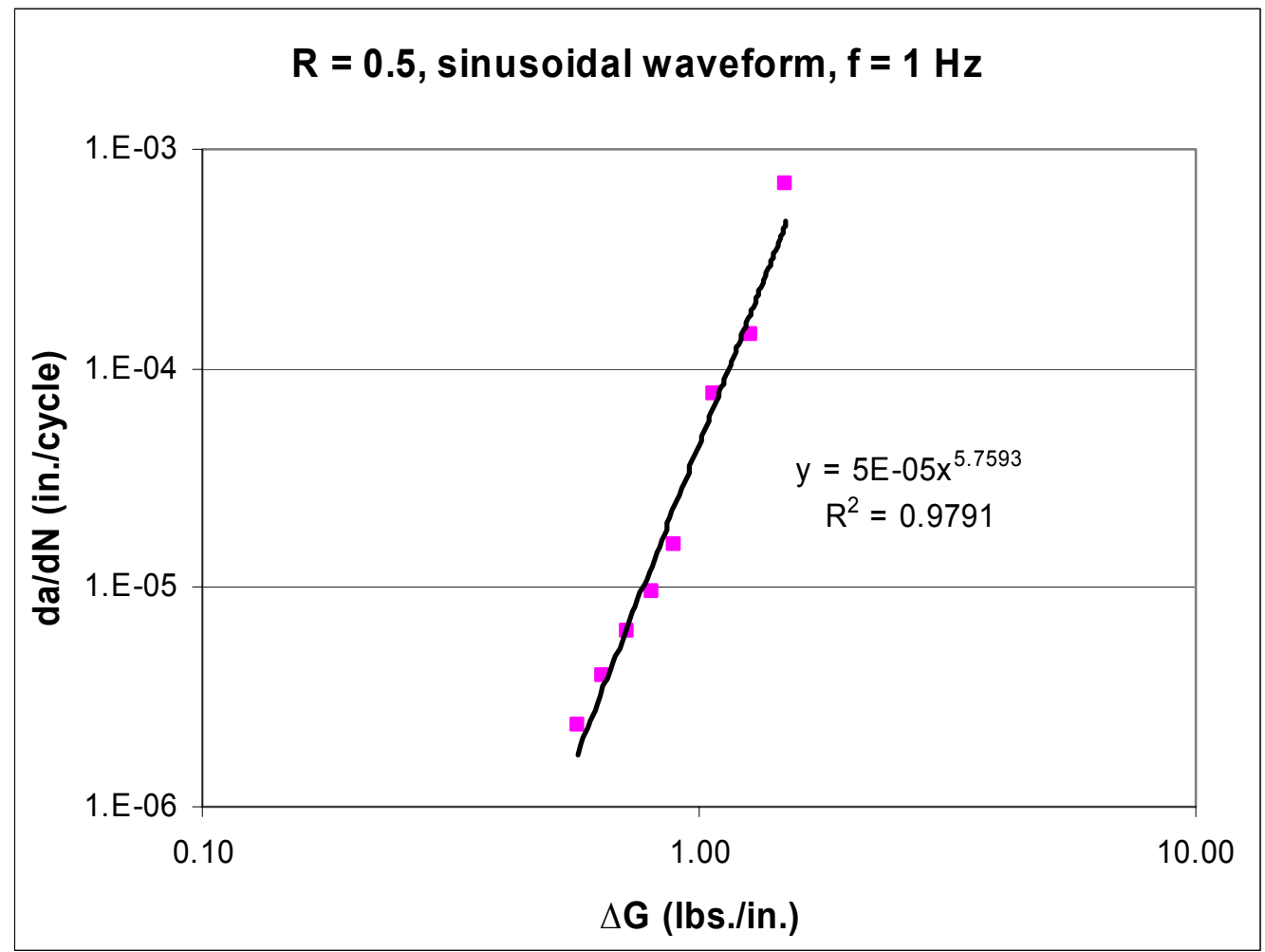

Fig. 4.23 The log-log plot of the crack propagation rate $d a / d N$ versus the strain energy release rate range $\Delta G$ for the sinusoidal waveform under a constant load ratio $R=0.5$ and a frequency $f=1 \mathrm{~Hz}$ 


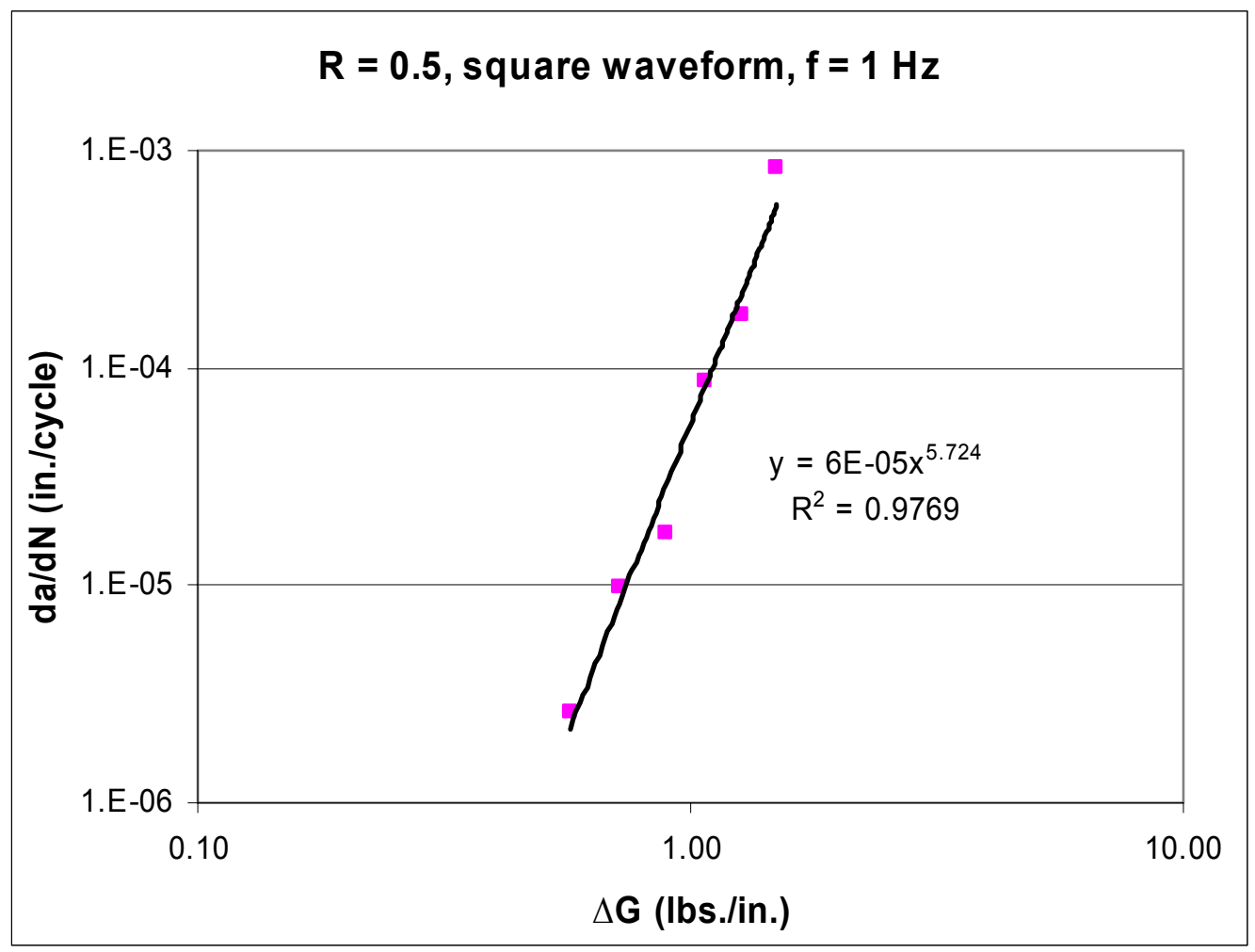

Fig. 4.24 The log-log plot of the crack propagation rate $d a / d N$ versus the strain energy release rate range $\Delta G$ for the square waveform under a constant load ratio $R=0.5$ and a frequency $f=1 \mathrm{~Hz}$ 


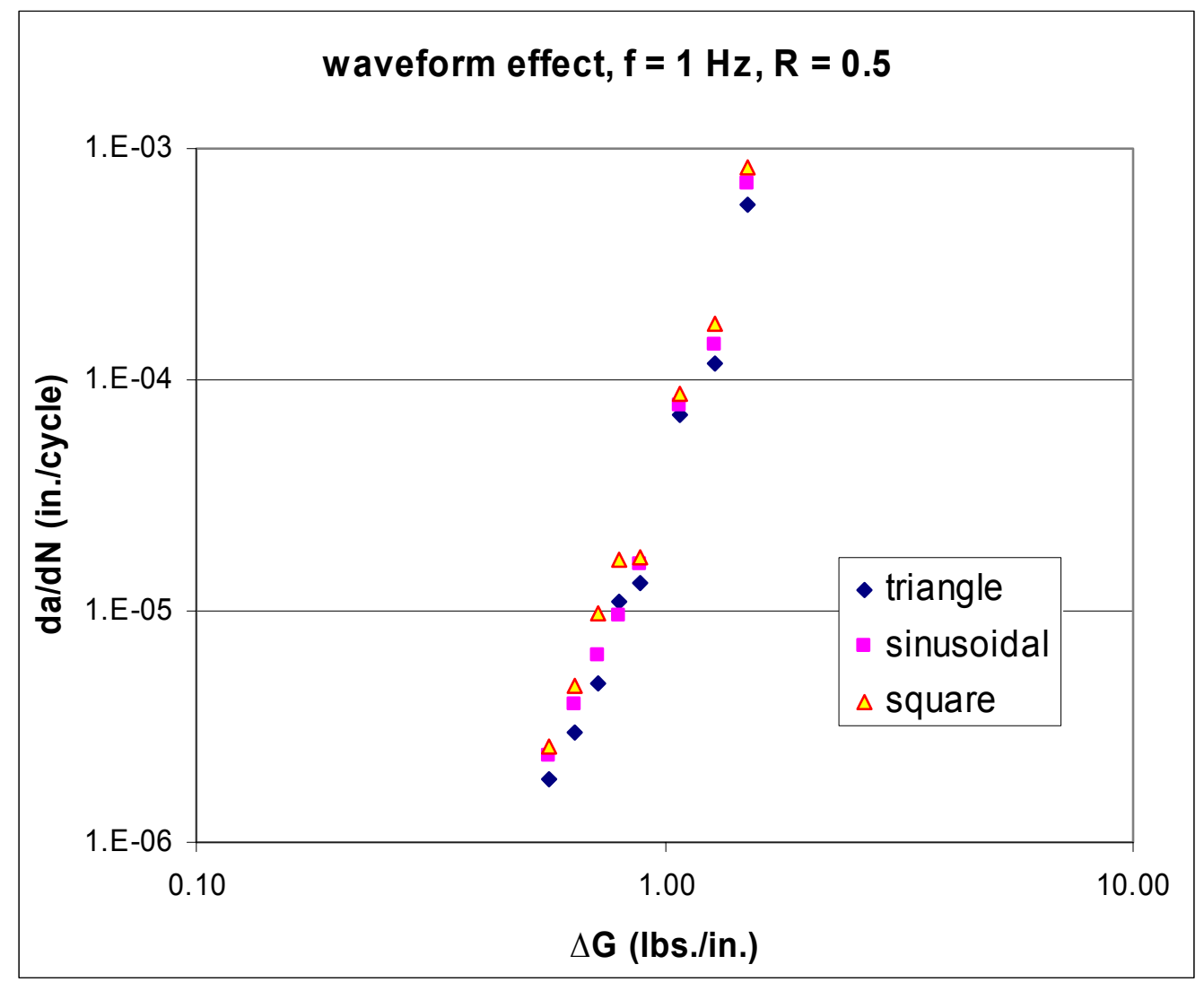

Fig. 4.25 The log-log plot of the crack propagation rate $d a / d N$ versus the strain energy release rate range $\Delta G$ for different waveforms under a constant load ratio $R=0.5$ and a frequency $f=1 \mathrm{~Hz}$ 


\subsubsection{The failure surface}

A typical fracture surface for the phenolic FRP-red maple wood CDCB specimen under different waveforms is displayed in Fig. 4.26. As observed in the tests, most of the surface fracture of phenolic FRP-red maple wood CDCB specimen happened in the interface adhesive. Similar to the failure surface under different load ratios, some of the fractured surfaces happened within the continuous strand mat (CSM) layer of the FRP substrate in combination with interface adhesive failure. For several specimens, significant bridging effect was also observed during crack propagation. Again, the data having bridging effect were disregarded in order to avoid their influence on the results. Failures around the loading pins and contoured portions of the CDCB specimens did not occur in the present tests. Again, It was observed that the interface bond failure was the primary failure mode for the phenolic FRP-red maple wood CDCB specimen under constant amplitude cyclic loading and three different waveforms. 


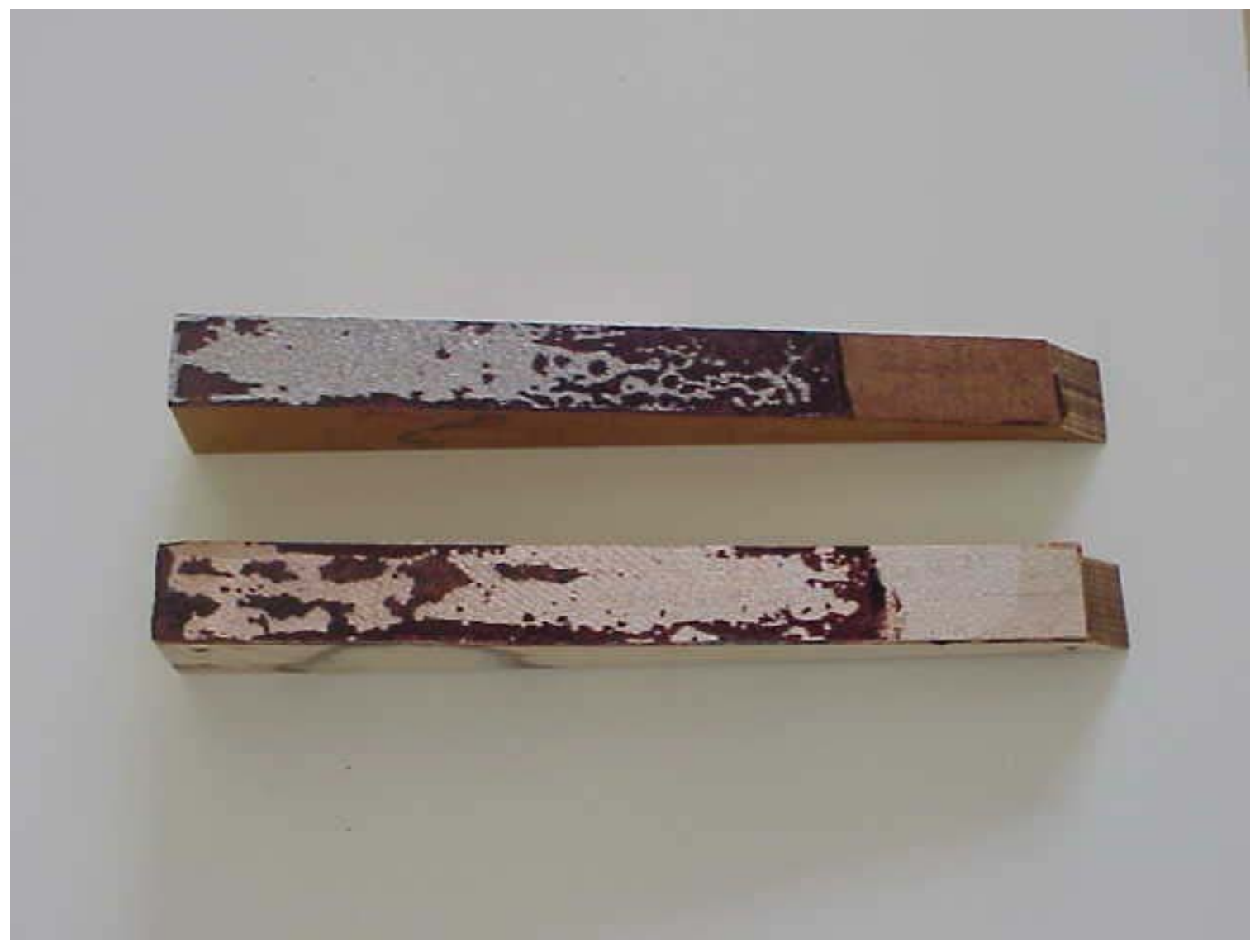

Fig. 4.26 A typical fracture surface for the phenolic FRP-red maple wood CDCB specimen under different waveforms 


\subsection{Conclusions}

The waveform effect on the crack propagation rate of the interface bond between red maple wood and phenolic FRP composite was studied in this chapter. Three sets of testing corresponding to triangular, sinusoidal and square waveforms were performed with a CDCB specimen. All the tests were carried out under constant amplitude cyclic loading at room temperature and laboratory conditions. The effects of three wave shapes on crack propagation rate were considered here and examined carefully. The results of this chapter are summarized as follows:

1. The CDCB specimen is shown to be suitable for the interface fatigue tests.

2. Three waveform shapes considered in this study had little effect on crack propagation rate of the interface under constant cyclic loading.

3. The bonded interface under constant cyclic loading exhibited a catastrophic ultimate failure mode.

4. The interface bond failure was the primary failure mode for the CDCB specimens under three different waveforms. 


\section{CHAPTER 5}

\section{FREQUENCY EFFECT}

\subsection{Introduction}

We have studied load ratio and waveform effect on crack propagation rate of phenolic FRP-red maple wood bonded interfaces in Chapters 3 and 4, respectively. It has been shown that the load ratio has significant effect, while waveform has negligible effect, on crack propagation rate of the bonded interfaces, in good agreement with previous work presented by others. However, with respect to the load frequency, there is a controversy on its importance on fatigue crack propagation rate. Some researchers sustain that the load frequency has little or negligible effect; for example, Singh et al. (2001) showed no appreciable effect of frequency on the crack growth rate of 2618 aluminum alloy silicon carbide metal matrix composite, using a single edge notched tensile specimen, even though some plastic deformation at the crack front was observed. In contrast, others report that the load frequency has substantial effect on crack growth rate under cyclic loading. A detailed literature review on the loading frequency effect for various materials is given in section 5.2.

In this chapter, three groups of tests for the loading frequency of $0.1,1.0$ and $10 \mathrm{~Hz}$ at constant load ratio of 0.5 and sinusoidal waveform are performed. The same materials and 
CDCB specimens as in Chapters 3 and 4 are used. This Chapter is structured as: (1) Literature review, (2) Fatigue test, (3) Results, and (4) Conclusions.

\subsection{Literature Review}

Solomon (1973) described the frequency effect by the following expression:

$$
C_{1} \equiv f^{k-1} N_{f}
$$

where, $C_{1}$ is the frequency modified fatigue life, $f$ is the frequency, $k$ is a constant, and $N_{f}$ is the number of cycles to failure. Following this concept, a modified crack growth expression was derived as

$$
\frac{d \ln a}{d N}=\Phi\left(\Delta \varepsilon_{p}\right)^{\alpha} f^{k-1}
$$

where, $a$ is the crack length, $\Delta \varepsilon_{p}$ is the plastic strain range of the test, $\Phi$ and $\alpha$ are constants. At a frequency below $0.05 \mathrm{cpm}$ for an iron based super-alloy A286 cycled at $1100^{\circ} \mathrm{F}$, the crack growth was time dependent (time to failure is a constant) with $k=0$; in this case, the crack propagation is ascribed to the stress rupture rather than fatigue. At higher frequencies with $k=0.55$, the crack growth behavior was both time and cycle dependent. Solomon explained this mixed time and cycle effect as the sum of pure time and pure cycle growth rates based on a linear superposition assumption, which did not 
agree with his experimental growth rate data, showing larger discrepancy at higher frequencies. He then tried a linear damage model, which combined both time and cycle dependent failure processes as

$$
\frac{N_{f}}{N_{f^{*}}}+\frac{t_{f}}{t_{f^{*}}}=1
$$

where $N_{f}$ and $t_{f}$ are, respectively, the number of cycles and the time to failure, and $N_{f^{*}}$ and $t_{f^{*}}$ are their corresponding quantities for pure cycle and time dependent behaviors, but this linear damage hypothesis model could not explain his experimental data either. Solomon attributed the deviation of experimental data from the superposition or cumulative damage methods to environmental influences. The absorption of oxygen and oxygen diffusion were thought to be the possible reasons that affected crack propagation rate for A286 under high temperature.

Mandell and Meier (1983) studied the frequency effect of tensile fatigue of $0^{\circ} / 90^{\circ}$ E-glass/epoxy laminates. Low frequencies of $0.01,0.1$ and $1.0 \mathrm{~Hz}$ were used to prevent an interaction caused by thermal and mechanical effects. The dumbbell specimen was used and fatigue tests were carried out in a load-controlled mode. A semi-log S-N curve was used to fit experimental data. They found that the degradation rate of Scotchply 1002 is about $10 \%$ of the single-cycle strength per decade of cycles. At approximately 125 Mpa and a $\log N$ of 6.2 , the rates of degradation for all three frequencies tended to converge. A notable feature of the frequency effect reported by them is that the specimen 
tested at higher frequency exhibited longer lifetimes over the entire test range. The maximum stress was plotted against both number of cycles to failure and also cumulative time to failure. A linear regression line was fitted very well to these two kinds of average $\log$ lifetime data. However, it can lead to confusion when number of cycles is used as a lifetime parameter under different frequencies, and therefore, one should be careful when using the number of cycles to failure as a lifetime parameter or at least clarify its physical meaning for the user.

Moskala (1993) studied the frequency effect on crack growth rate in a miscible amorphous blend of polycarbonate and copolyester. Compact tension specimens were tested at frequencies from $0.25 \mathrm{~Hz}$ to $25 \mathrm{~Hz}$. He reported that the fatigue resistance of the neat blend is not affected by frequencies, whereas the fatigue resistance of the toughened blend increases with increasing frequency. A thermograph camera was used to monitor crack tip temperature. His test results showed that the crack tip is the warmest region of the specimen, although the temperature increase was only a modest $8^{\circ} \mathrm{C}$. He concluded that the creep-induced crack growth, not the hysteretic heating, is the key factor for the frequency sensitivity of the toughened blends.

Tong and Byrne (1999) studied the frequency effect on fatigue crack growth rate in a pre-alloyed powder material Udimet $720 \mathrm{Li}$ at $650^{\circ} \mathrm{C}$. Standard compact tension specimens were used under constant $\Delta K$ control, which provides unambiguous linearity of crack growth rate at a particular frequency. Three regimes of frequency effect were studied: (a) cycle dependent regime, (b) mixed time/cycle dependent regime, and (c) time dependent regime. Cycle dependent regime was represented by crack growth at room 
temperature, mixed time/cycle dependent regime was approximated by fatigue data at frequencies from 0.001 to $5.0 \mathrm{~Hz}$ at $650^{\circ} \mathrm{C}$, and time dependent crack growth was obtained under sustained loads at $650^{\circ} \mathrm{C}$. Mixed time/cycle dependent regime was found to be the dominant mechanism in their study. A phenomenological Paris Law equation was used and expressed as

$$
\frac{d a}{d N}=g(f) C \Delta K^{m}
$$

where, the constants $C$ and $m$ were obtained from room temperature data. A transition frequency, below which frequency starts to gain significance, $f_{m c}$ was introduced to rewrite the above equation as

$$
\frac{d a}{d N}=C\left(\frac{f_{m c}}{f}\right)^{\alpha} \Delta K^{m}
$$

They argue that the transition frequency decreases as the stress intensity increases. The transition frequency was defined as

$$
f_{m c}=B \Delta K^{n}
$$

where, $\mathrm{n}$ was calculated as -3.7 , which is close to -3.2 for data obtained for IN718. Agreement between experimental and predicted results in the mixed regime was achieved at frequencies from 0.001 to $1.0 \mathrm{~Hz}$. The mapping of results for $\Delta K=30 \mathrm{Mpa}(\mathrm{m})^{0.5}$ was 
extended to stress intensity range of 25 to $60 \mathrm{Mpa}(\mathrm{m})^{0.5}$. From compiling data for several other Ni-based super-alloys, Tong and Byrne suggested that the change of crack growth rate is not sensitive for these materials.

Sun and Chan (1979) evaluated the load frequency effect on fatigue life of notched laminated graphite/epoxy composites. Fatigue tests were conducted using constant amplitude tension-tension sinusoidal load cycles. Three loading levels (maximum stress amounted to 66.6, 57.7 and 53.3 percent of the average ultimate failure stress) and four frequencies $(1,3,10$ and $30 \mathrm{~Hz})$ with a load ratio $R=1 / 15$ were used for center hole specimens. Two thermocouples attached to a specimen were used to measure temperature changes continuously. The fatigue test data were presented in the form of plots of fatigue life (number of cycles to failure) versus frequencies, and they observed that within the frequency range a peak fatigue life existed, which tended to shift to higher frequency for lower load levels. The temperature increase near the hole was recorded, and higher load and frequency produced higher temperature as expected. A pronounced temperature increase was noticed at $66.6 \%$ load level. This may be the reason that the fatigue life at this load level declined for a frequency of about $3 \mathrm{~Hz}$. No appreciable stiffness change from fatigue damage was reported. From the fatigue failure surface, they found no obvious failure mode changing for different frequencies. Based on Schapery's theory for creep crack propagation in visco-elastic media and to include the significant non-creep effect, a modified theoretical model to predict fatigue life as a function of frequency, $N(\omega)$, was presented as 


$$
N(\omega)=N\left(\omega_{1}\right) \frac{1+\alpha\left(\omega_{1}\right)}{1+\frac{\omega_{1}}{\omega} \alpha\left(\omega_{1}\right)}
$$

with $\alpha\left(\omega_{1}\right)$ representing crack extension per cycle due to creep at frequency $\omega_{1}$ as:

$$
\alpha\left(\omega_{1}\right)=\left[1-\frac{N\left(\omega_{1}\right)}{N\left(\omega_{2}\right)}\right] /\left[\frac{N\left(\omega_{1}\right)}{N\left(\omega_{2}\right)}-\frac{\omega_{1}}{\omega_{2}}\right]
$$

where, $N\left(\omega_{1}\right)$ and $N\left(\omega_{2}\right)$ are the fatigue lives at frequencies $\omega_{1}$ and $\omega_{2}$, respectively. This model did not consider temperature effect. Considering the frequency and temperature effects, the modified equation was given as

$$
N(\omega)=N\left(\omega_{1}\right) \frac{\omega}{\omega_{1}} e^{\eta}\left[\left(\Delta T_{1}-\Delta T\right) / T_{0}\right]
$$

where, $T_{0}$ is the room temperature, $\Delta T$ is the near-hole temperature increase at half-life of the composite, and $\eta$ is a parameter. This model with $\eta \approx 9$ gives a good agreement with experimental results and accounts for both frequency and temperature effects.

Arad et al. (1972) studied the frequency effect on the crack growth rate of Nylon 6.6. The frequencies of $0.1,5$ and $20 \mathrm{~Hz}$ were considered. They found that unlike metals, there was a marked change for the crack growth rate of polymeric materials as the loading frequency was altered. The crack growth rate with respect to number of cycles 
tended to decrease as the frequency was increased. However, the crack growth rate with respect to time tended to increase as the frequency was increased.

\subsection{Fatigue Test}

This section describes materials, test specimen, and procedures.

\subsubsection{Material and specimen}

A contoured double cantilever beam (CDCB) specimen designed by the RayleighRitz method was used for fatigue tests of red maple wood-phenolic FRP composite interfaces. The CDCB specimen is contoured to achieve a constant rate of compliance change with respect to crack length, $d C / d a$. The details of the specimen design and applications for wood-FRP interface fracture studies are given in several papers by Davalos et al. (1997, 1998a, 1998b, 2000a, 2000b) and Qiao et al. (2000). The geometric details of the CDCB specimen in this study are shown in Fig. 2.4, and the adherends consist of red maple wood and pultruded phenolic FRP with the contoured portions made of laminated veneer lumber (LVL). The lay-up of the pultruded phenolic FRP laminate is shown in Figure 2.3, and the mechanical properties of red maple and phenolic FRP are summarized in Table 2.2. The adhesive used for bonding wood-FRP interface was Resorcinol-Formaldehyde from INDSPEC Chemical Corp., Pittsburgh, PA. 
The average of the $d C / d a$ value obtained by the Rayleigh-Ritz method and finite element modeling was $27.17 \times 10^{-5} \mathrm{lb}^{-1}$. Compliance calibration tests were conducted for several wood-FRP CDCB samples, and the average $d C / d a$ value obtained from experiments was $29.59 \times 10^{-5} \mathrm{lb}^{-1}$. The experimental result of the $d C / d a$ value is used to calculate strain energy release rate $G$ in the present study.

\subsubsection{Test procedure}

Cyclic tension-tension fatigue tests were conducted in a computer controlled MTS servo hydraulic machine. The frequency was kept constant during the testing of each group of samples. Three groups of tests for frequency $f$ of $0.1,1.0$ and $10 \mathrm{~Hz}$ were completed under load control mode (see Fig. 5.1). The applied loads for each group of tests are listed in Tables 5.1, 5.2 and 5.3. A load ratio $R$ of 0.5 and a sinusoidal waveform were used for all the tests. The tests in the MTS machine were controlled by preprogrammed software, which was also used as a data acquisition system. Since only the relative value of crack opening displacement (COD) is needed, a crosshead displacement instead of an extensometer displacement was used to measure the COD. The test environment was room temperature and open laboratory conditions for all the samples. 
Table 5.1 Applied load for frequency of $f=0.1 \mathrm{~Hz}$ under constant load ratio $R=0.5$ and sinusoidal waveform

\begin{tabular}{|c|c|c|c|c|}
\hline $\boldsymbol{P}_{\min }$ & $\boldsymbol{P}_{\max }$ & $\Delta \boldsymbol{P}=\boldsymbol{P}_{\max }-\boldsymbol{P}_{\min }$ & $\boldsymbol{R}$ & $\boldsymbol{P}_{\text {mean }}$ \\
\hline 65 & 130 & 65 & 0.5 & 97.5 \\
\hline 60 & 120 & 60 & 0.5 & 90 \\
\hline 55 & 110 & 55 & 0.5 & 82.5 \\
\hline 50 & 100 & 50 & 0.5 & 75 \\
\hline 45 & 90 & 45 & 0.5 & 67.5 \\
\hline 40 & 80 & 40 & 0.5 & 60 \\
\hline
\end{tabular}

Table 5.2 Applied load for frequency of $f=1 \mathrm{~Hz}$ under constant load ratio $R=0.5$ and sinusoidal waveform

\begin{tabular}{|c|c|c|c|c|}
\hline $\boldsymbol{P}_{\boldsymbol{m i n}}$ & $\boldsymbol{P}_{\max }$ & $\Delta \boldsymbol{P}=\boldsymbol{P}_{\max }-\boldsymbol{P}_{\min }$ & $\boldsymbol{R}$ & $\boldsymbol{P}_{\text {mean }}$ \\
\hline 65 & 130 & 65 & 0.5 & 97.5 \\
\hline 60 & 120 & 60 & 0.5 & 90 \\
\hline 55 & 110 & 55 & 0.5 & 82.5 \\
\hline 50 & 100 & 50 & 0.5 & 75 \\
\hline 47.5 & 95 & 47.5 & 0.5 & 71.25 \\
\hline 45 & 90 & 45 & 0.5 & 67.5 \\
\hline 42.5 & 85 & 42.5 & 0.5 & 63.75 \\
\hline 40 & 80 & 40 & 0.5 & 60 \\
\hline
\end{tabular}


Table 5.3 Applied load for frequency of $f=10 \mathrm{~Hz}$ under constant load ratio $R=0.5$ and sinusoidal waveform

\begin{tabular}{|c|c|c|c|c|}
\hline $\boldsymbol{P}_{\min }$ & $\boldsymbol{P}_{\max }$ & $\Delta \boldsymbol{P}=\boldsymbol{P}_{\max }-\boldsymbol{P}_{\min }$ & $\boldsymbol{R}$ & $\boldsymbol{P}_{\text {mean }}$ \\
\hline 65 & 130 & 65 & 0.5 & 97.5 \\
\hline 60 & 120 & 60 & 0.5 & 90 \\
\hline 55 & 110 & 55 & 0.5 & 82.5 \\
\hline 50 & 100 & 50 & 0.5 & 75 \\
\hline 45 & 90 & 45 & 0.5 & 67.5 \\
\hline 40 & 80 & 40 & 0.5 & 60 \\
\hline
\end{tabular}




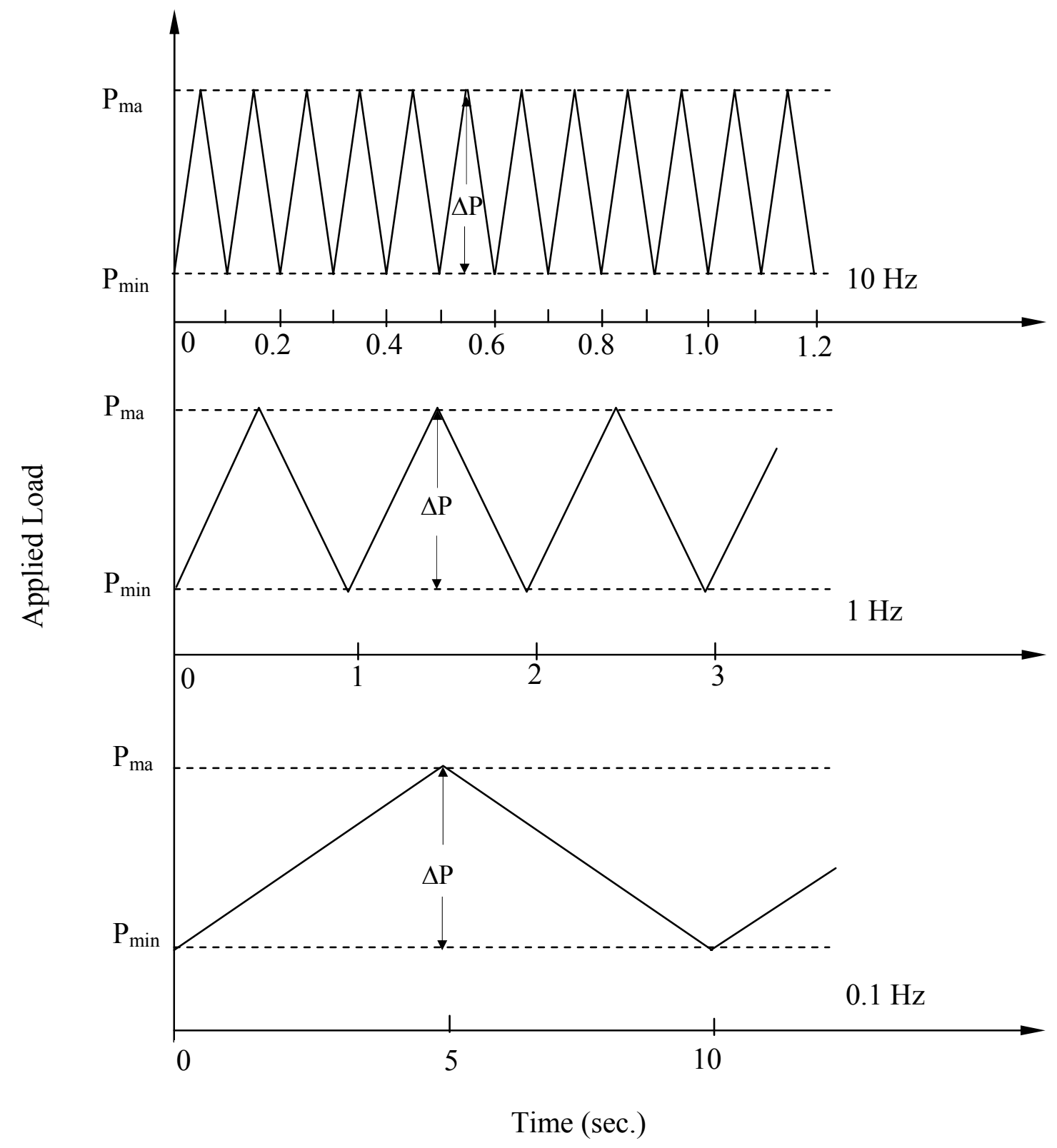

Fig. 5.1 A schematic illustration of cyclic loading at different triangular frequencies 


\section{$5.4 \quad$ Results}

This section presents data processing, crack growth rate by compliance method, modified Paris Law models, and description of failure surfaces.

\subsubsection{Experimental data processing}

The experimental results of the crack opening displacement (COD) versus the number of cycles for phenolic FRP-red maple wood bonded interface samples are summarized and discussed in this section. The data acquisition and processing are the same as those in section 3.4.1. As noticed by many researchers, fracture fatigue failure is of catastrophic nature, and the phenolic FRP-red maple wood bonded interface under three different frequencies also showed sudden ultimate failure. Twenty-four specimens were fabricated and then tested to obtain the COD propagation rate for different frequencies. The loading details are given in Tables 5.1, 5.2 and 5.3. For frequency of 1 $\mathrm{Hz}$ tests, the details are given in Chapter 3, and the same results of the COD versus number of cycles for frequency of $1 \mathrm{~Hz}$ were adopted in this section. The COD versus number of cycles of the tests for frequencies of 0.1 and $10 \mathrm{~Hz}$ are shown in Figs. 5.2 through 5.13. Since only the intermediate region of fatigue process is of concerned, Figs. 5.2 through 5.13 show the stable region of COD propagation versus number of cycles. The COD propagation rates for these specimens are listed in Tables 5.4, 5.5 and 5.6, corresponding to frequencies of $0.1,1.0$ and $10 \mathrm{~Hz}$. 


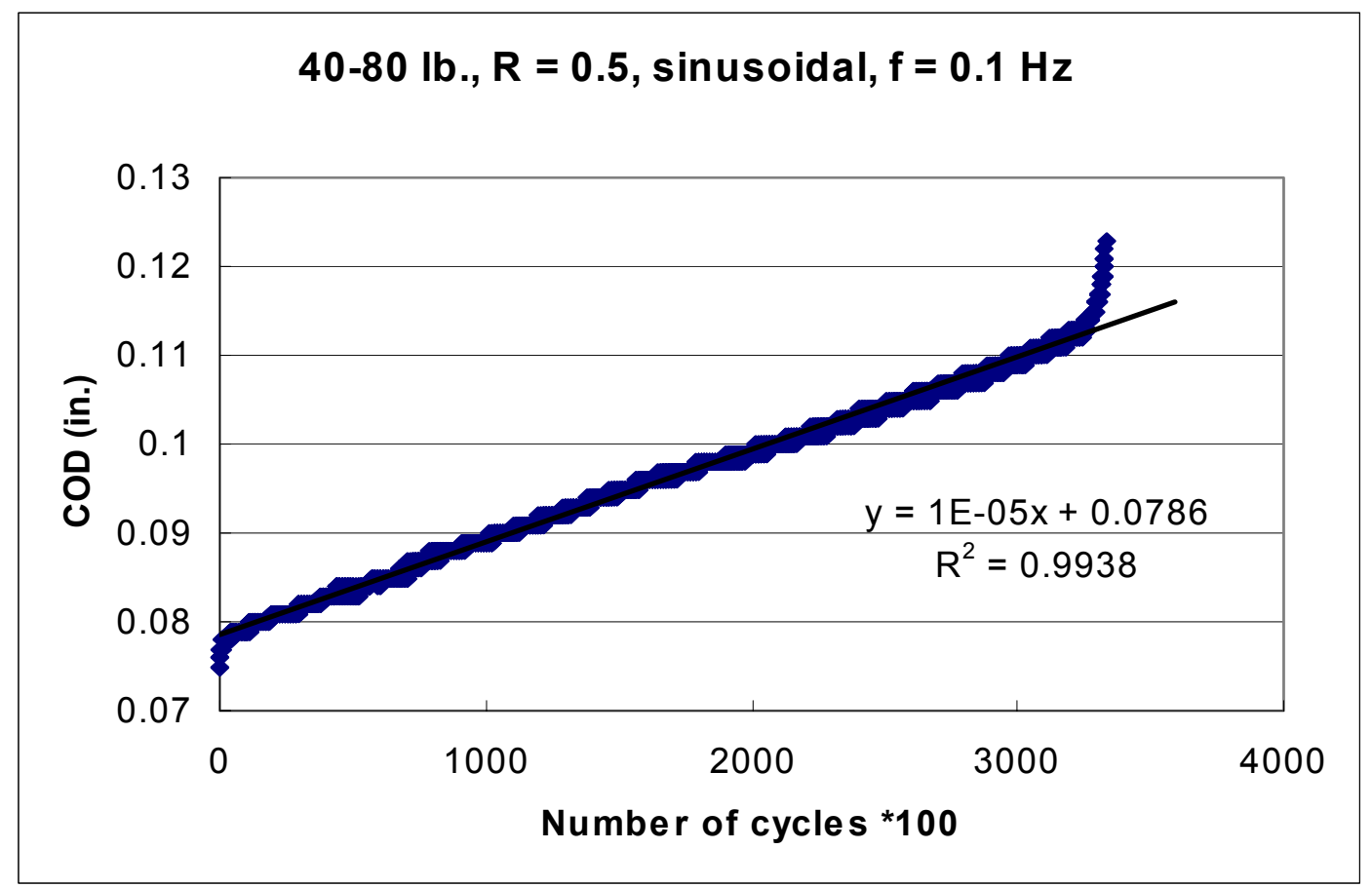

Fig. 5.2 COD versus number of cycles for a CDCB specimen under 40-80 lb. cyclic loading with a frequency $f=0.1 \mathrm{~Hz}$, load ratio $R=0.5$ and sinusoidal waveform 


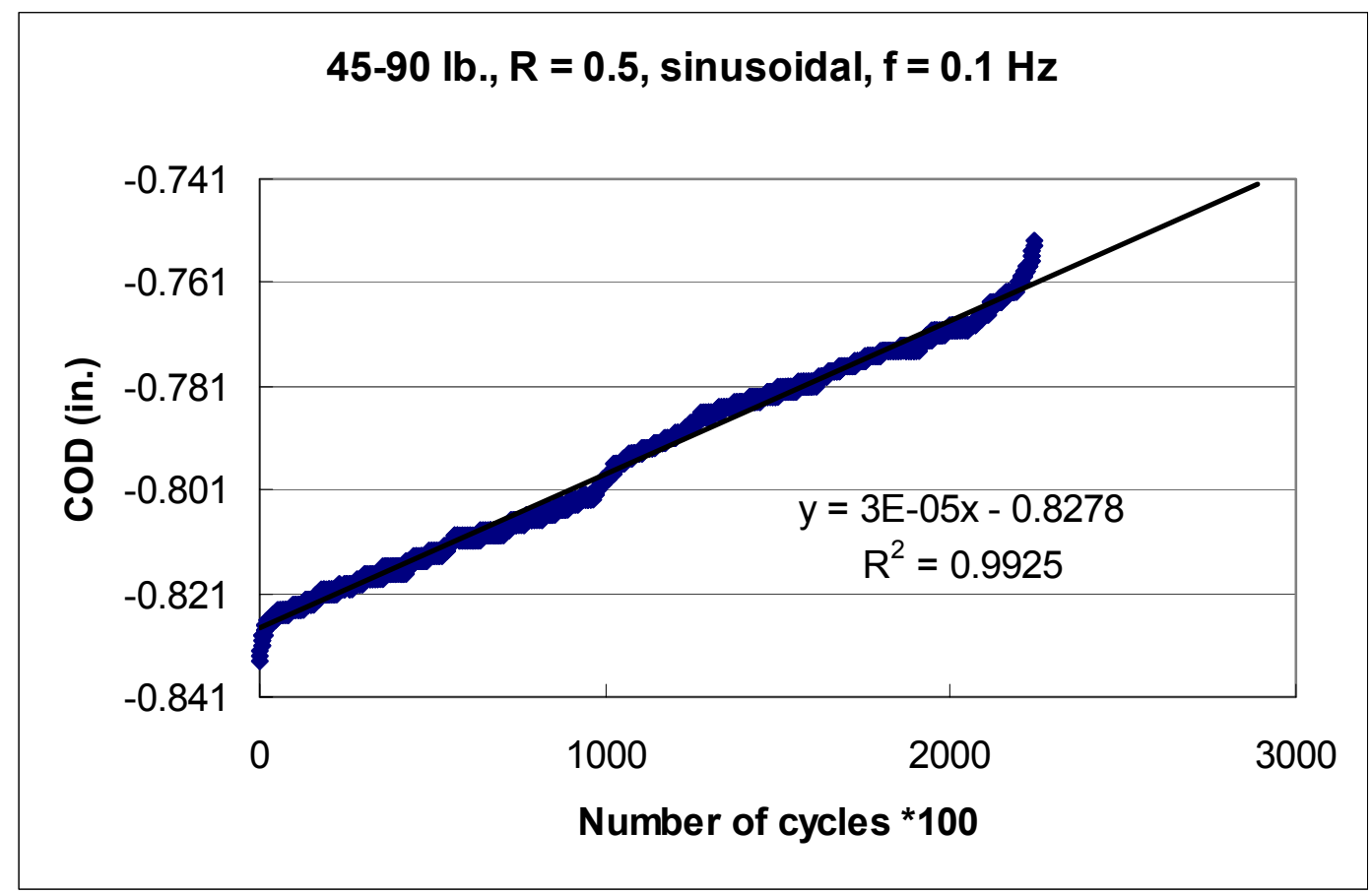

Fig. 5.3 COD versus number of cycles for a CDCB specimen under $45-90 \mathrm{lb}$. cyclic loading with a frequency $f=0.1 \mathrm{~Hz}$, load ratio $R=0.5$ and sinusoidal waveform 


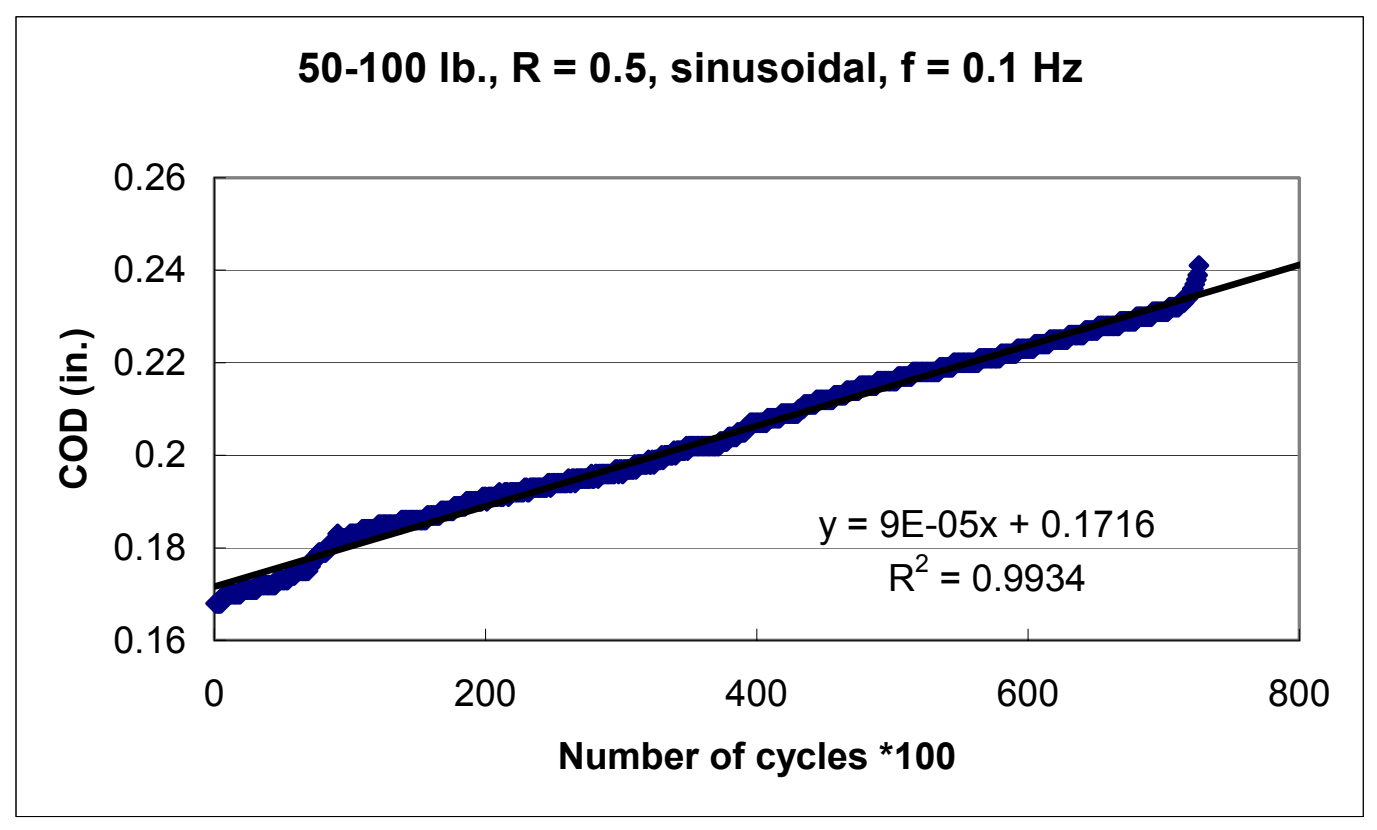

Fig. 5.4 COD versus number of cycles for a CDCB specimen under 50-100 lb. cyclic loading with a frequency $f=0.1 \mathrm{~Hz}$, load ratio $R=0.5$ and sinusoidal waveform 


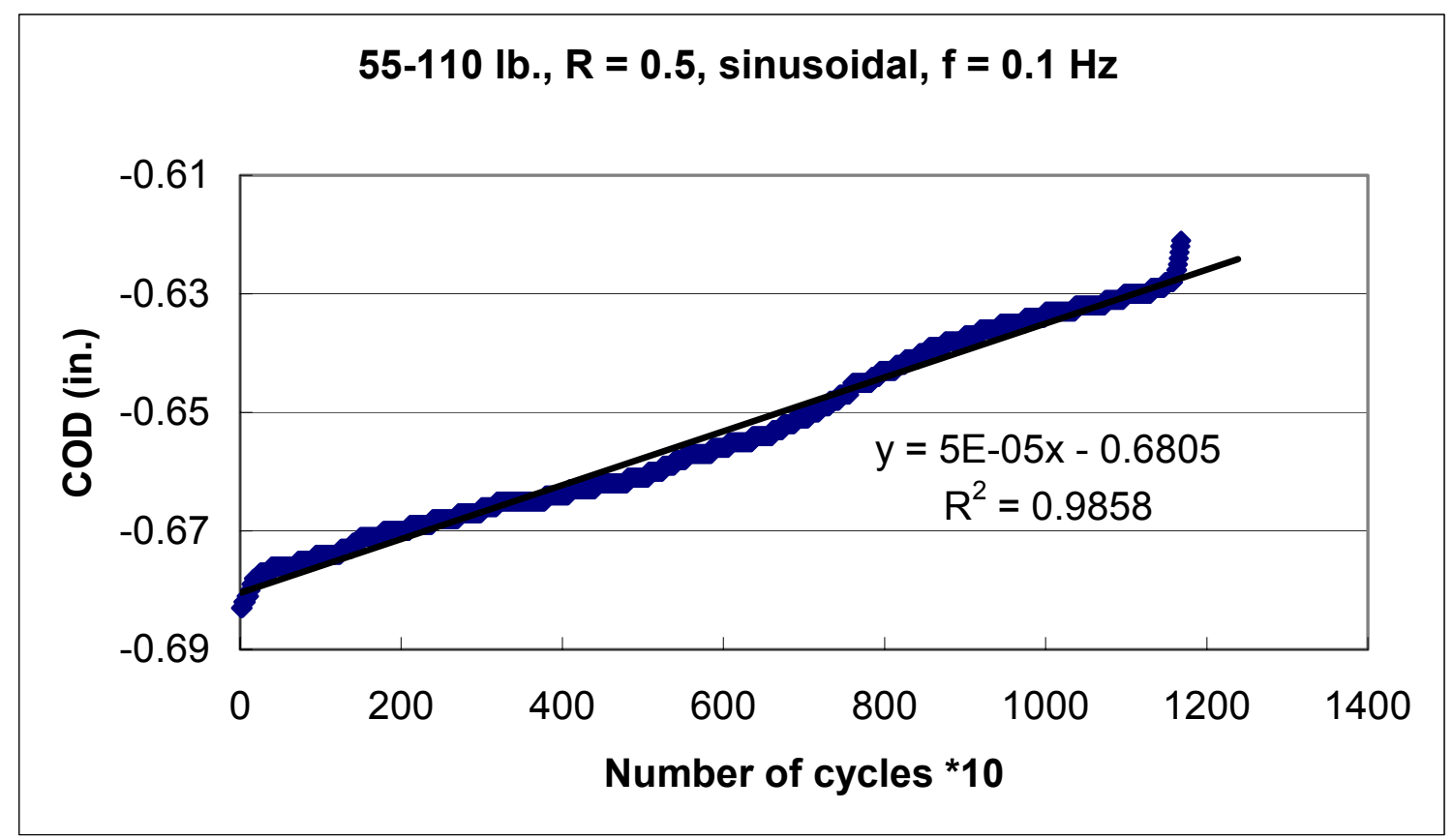

Fig. 5.5 COD versus number of cycles for a CDCB specimen under 55-110 lb. cyclic loading with a frequency $f=0.1 \mathrm{~Hz}$, load ratio $R=0.5$ and sinusoidal waveform 


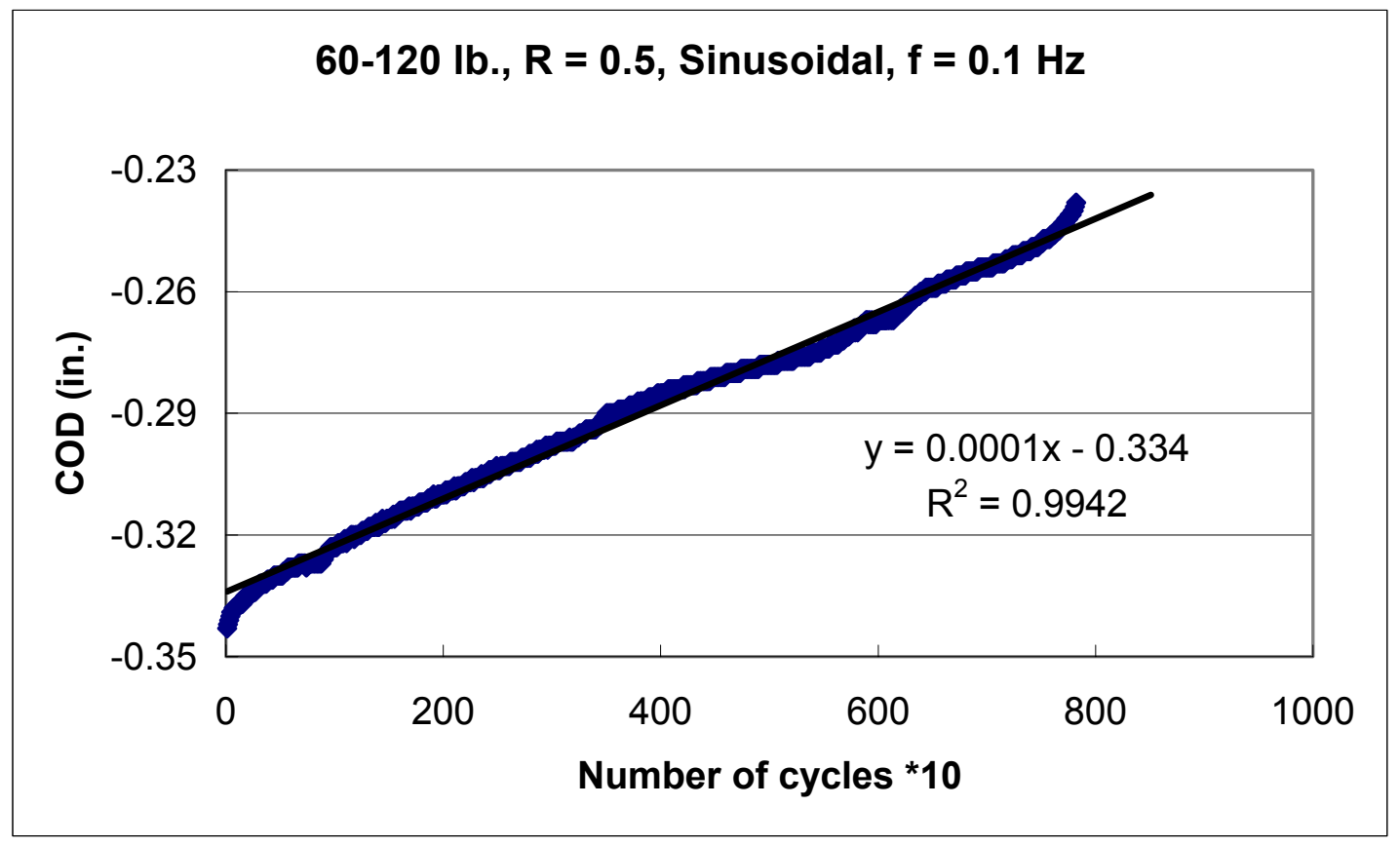

Fig. 5.6 COD versus number of cycles for a CDCB specimen under 60-120 lb. cyclic loading with a frequency $f=0.1 \mathrm{~Hz}$, load ratio $R=0.5$ and sinusoidal waveform 


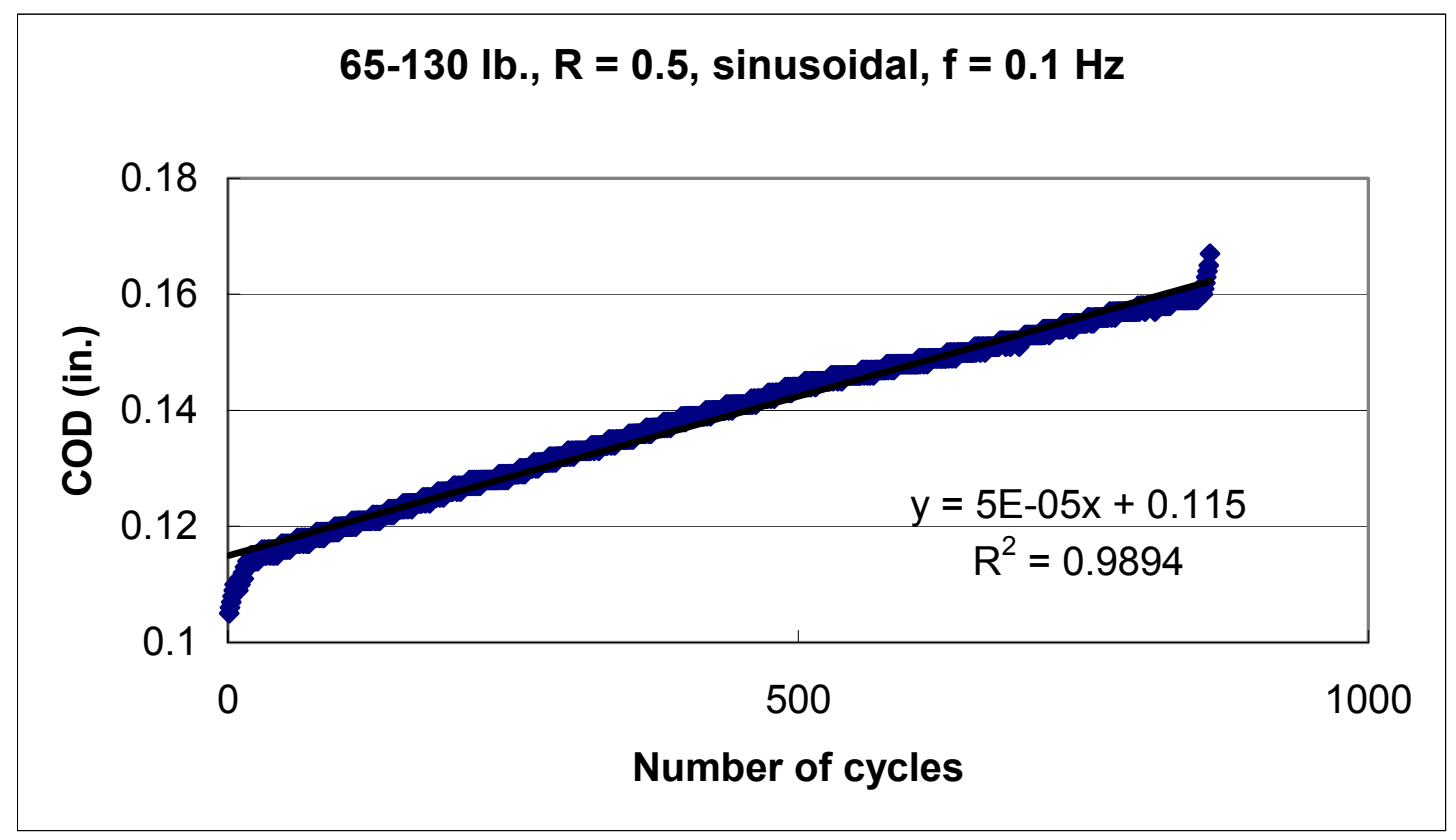

Fig. 5.7 COD versus number of cycles for a CDCB specimen under 65-130 lb. cyclic loading with a frequency $f=0.1 \mathrm{~Hz}$, load ratio $R=0.5$ and sinusoidal waveform 


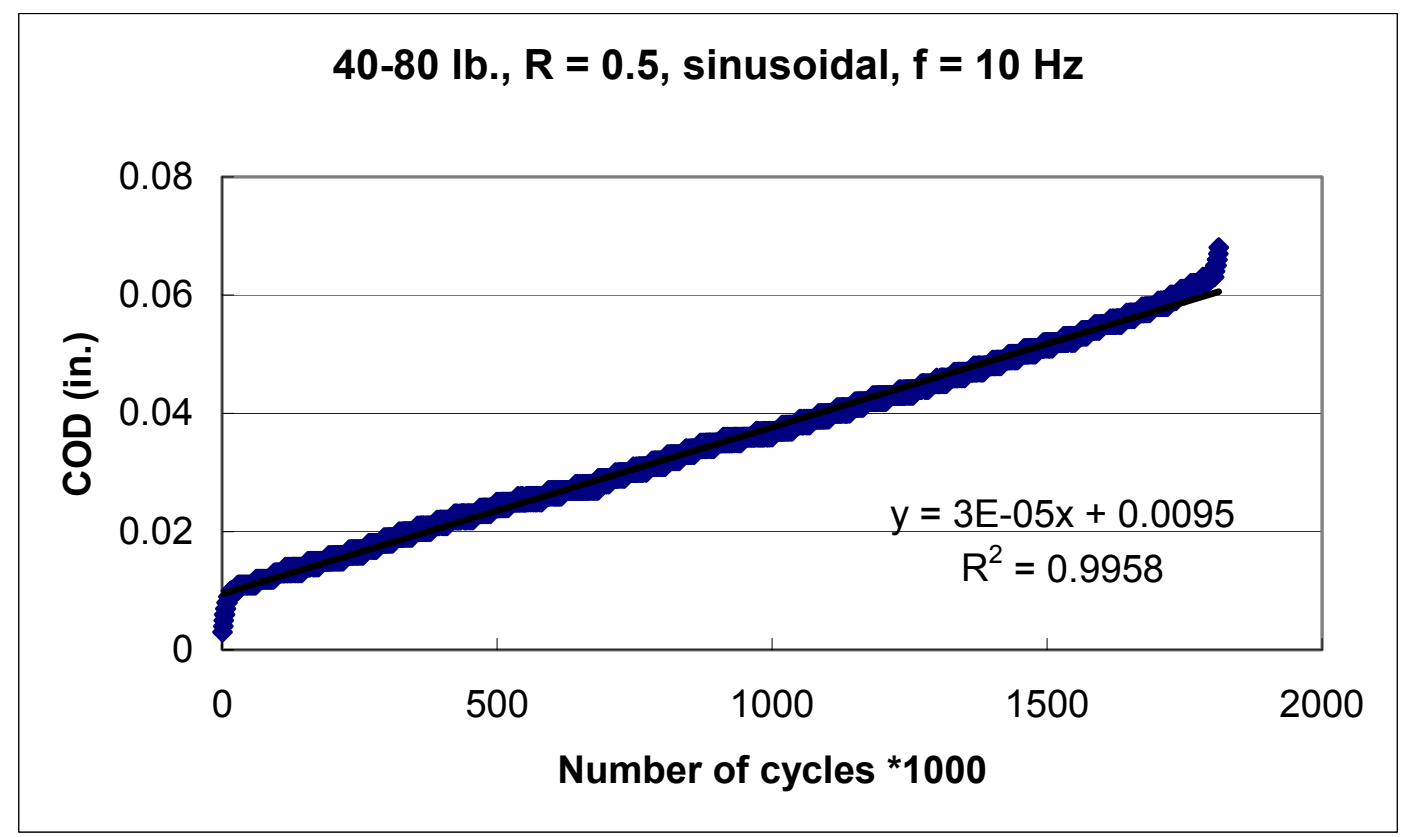

Fig. 5.8 COD versus number of cycles for a CDCB specimen under 40-80 lb. cyclic loading with a frequency $f=10 \mathrm{~Hz}$, load ratio $R=0.5$ and sinusoidal waveform 


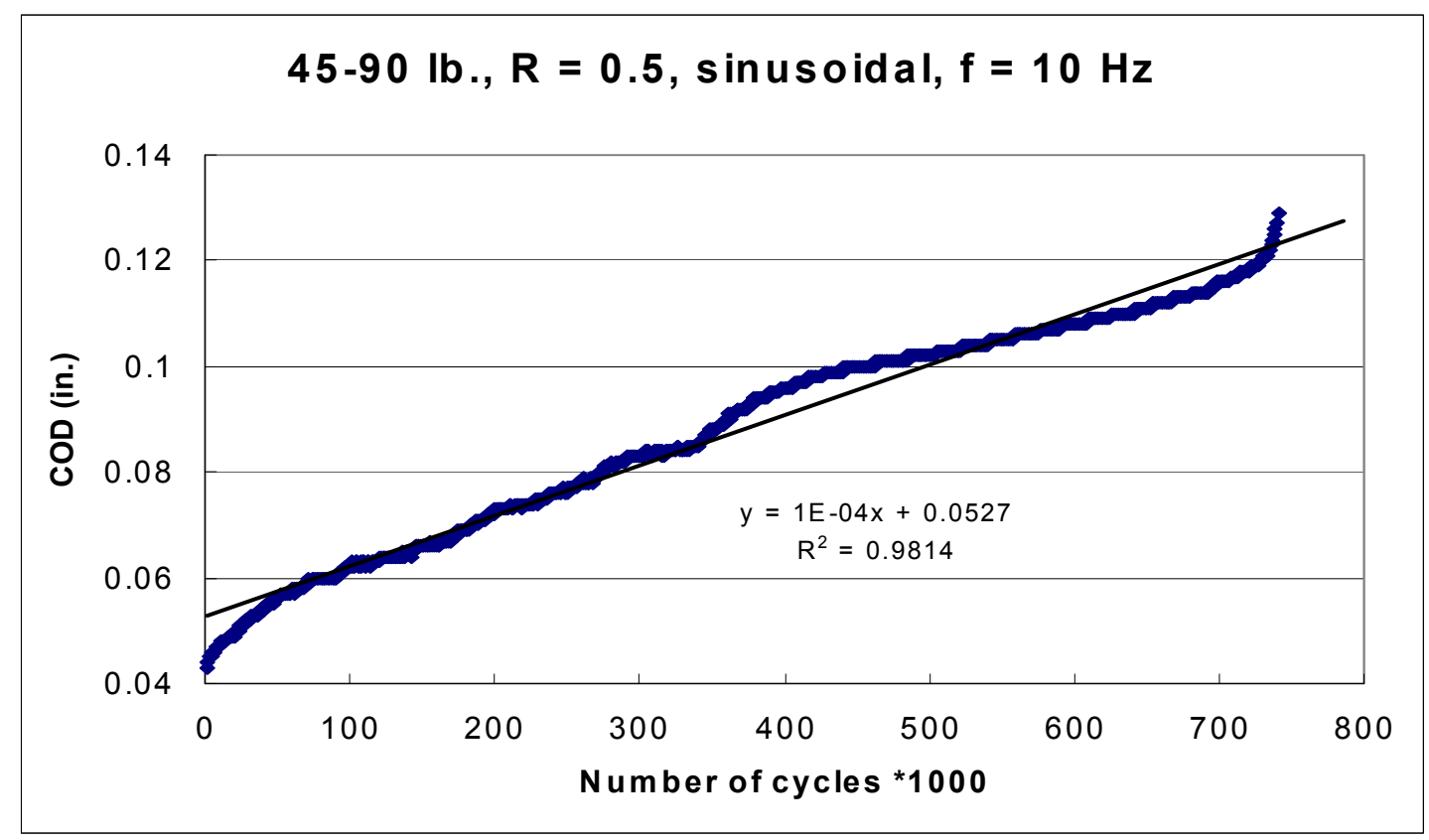

Fig. 5.9 COD versus number of cycles for a CDCB specimen under 45-90 lb. cyclic loading with a frequency $\mathrm{f}=10 \mathrm{~Hz}$, load ratio $\mathrm{R}=0.5$ and sinusoidal waveform 


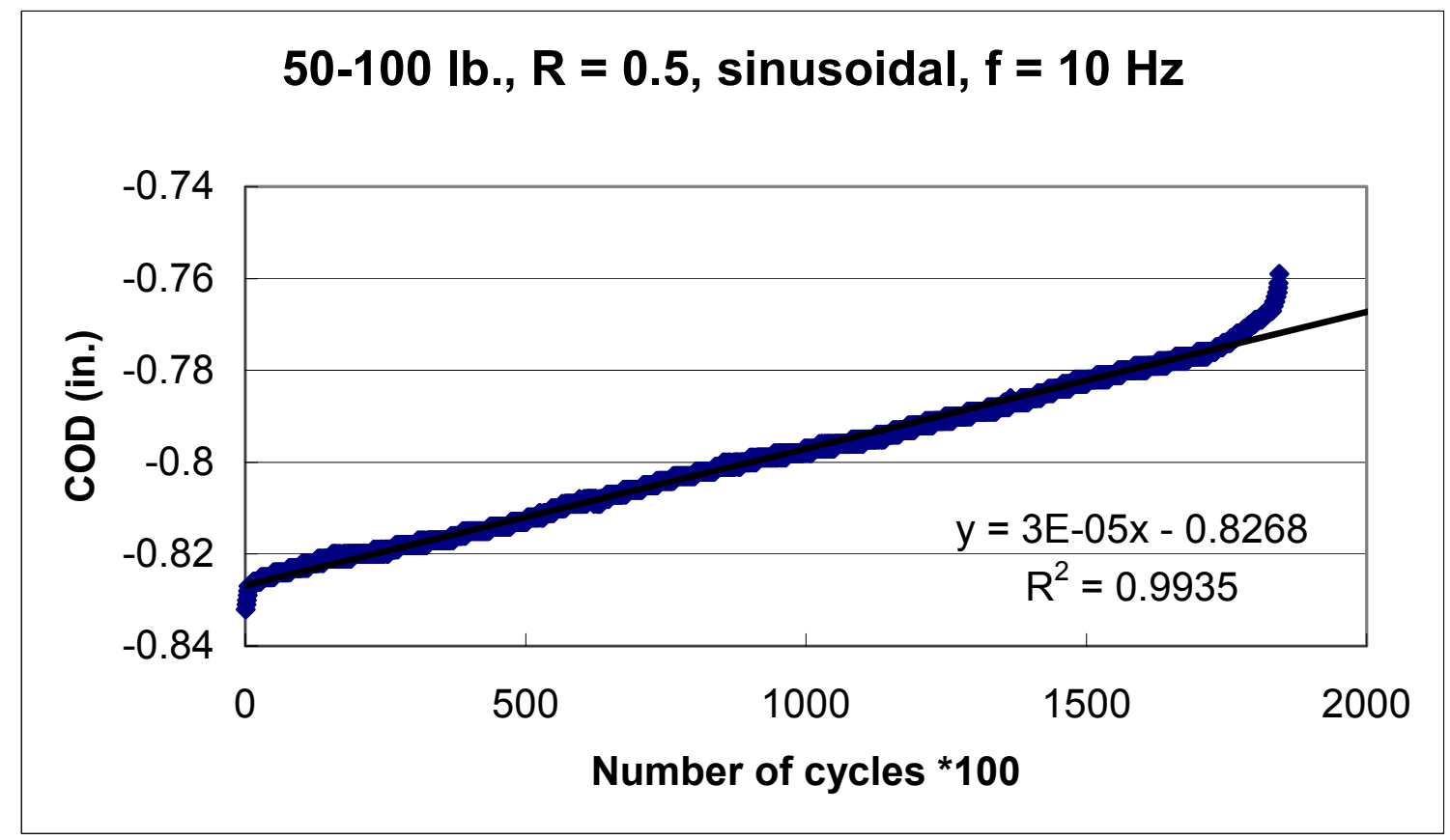

Fig. 5.10 COD versus number of cycles for a CDCB specimen under 50-100 lb. cyclic loading with a frequency $f=10 \mathrm{~Hz}$, load ratio $R=0.5$ and sinusoidal 


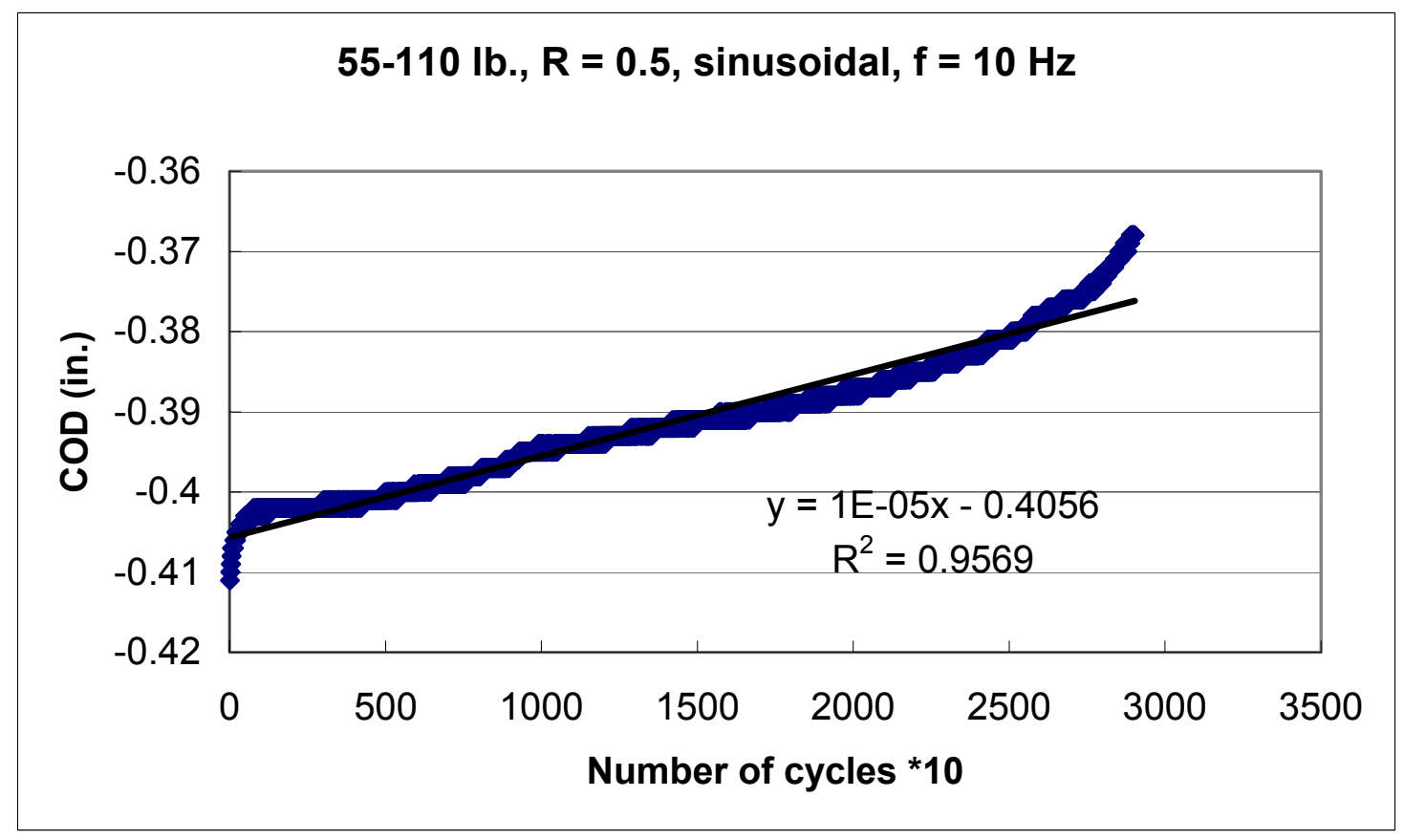

Fig. 5.11 COD versus number of cycles for a CDCB specimen under 55-110 lb. cyclic loading with a frequency $f=10 \mathrm{~Hz}$, load ratio $R=0.5$ and sinusoidal 


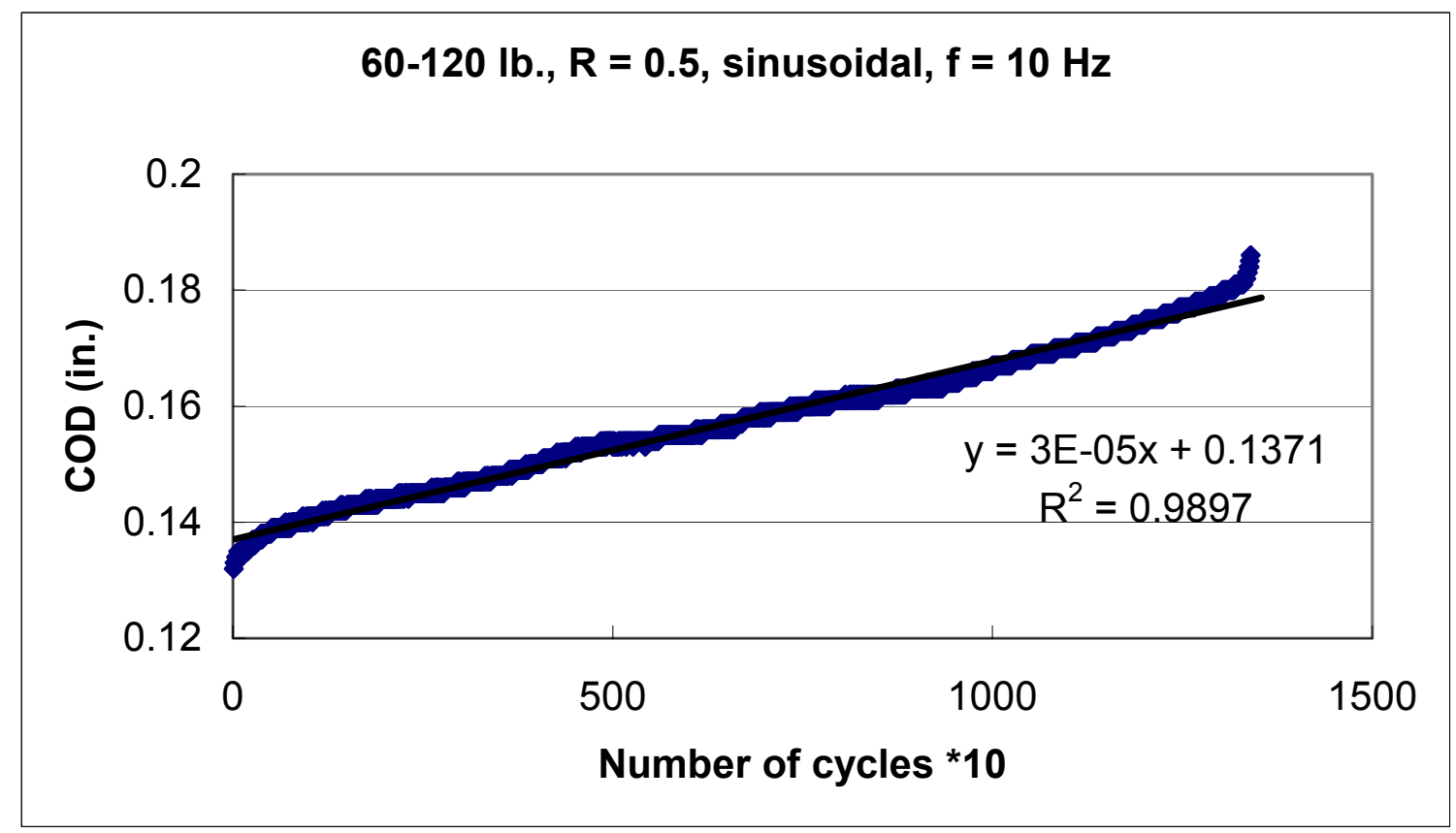

Fig. 5.12 COD versus number of cycles for a CDCB specimen under 60-120 lb. cyclic loading with a frequency $f=10 \mathrm{~Hz}$, load ratio $R=0.5$ and sinusoidal 


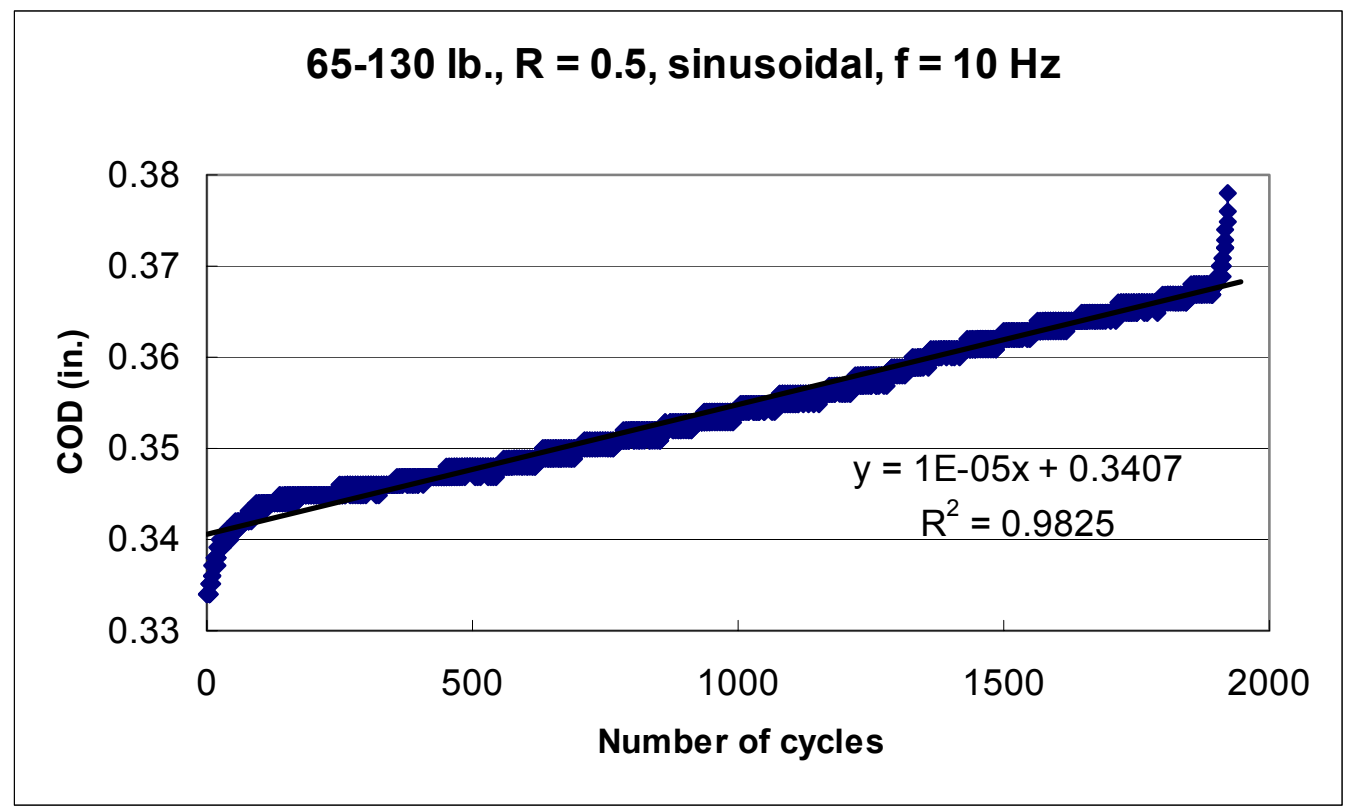

Fig. 5.13 COD versus number of cycles for a CDCB specimen under 65-130 lb. cyclic loading with a frequency $f=10 \mathrm{~Hz}$, load ratio $R=0.5$ and sinusoidal 
Table 5.4 Experimental results of COD propagation rate for frequency $f=0.1 \mathrm{~Hz}$ under constant load ratio $R=0.5$ and sinusoidal waveform

\begin{tabular}{ccc}
\hline$P_{\max }(\mathrm{lb})$. & $P_{\min }(\mathrm{lb})$. & $d C O D / d N($ in./cycle $)$ \\
130 & 65 & $5.00 \mathrm{E}-05$ \\
120 & 60 & $1.00 \mathrm{E}-05$ \\
110 & 55 & $5.00 \mathrm{E}-06$ \\
100 & 50 & $9.00 \mathrm{E}-07$ \\
90 & 45 & $3.00 \mathrm{E}-07$ \\
80 & 40 & $1.00 \mathrm{E}-07$ \\
\hline
\end{tabular}

Table 5.5 Experimental results of COD propagation rate for frequency $f=1 \mathrm{~Hz}$ under constant load ratio $R=0.5$ and sinusoidal waveform

\begin{tabular}{ccc}
\hline$P_{\max }(\mathrm{lb})$. & $P_{\min }(\mathrm{lb})$. & $d C O D / d N($ in./cycle $)$ \\
130 & 65 & $3.00 \mathrm{E}-05$ \\
120 & 60 & $5.00 \mathrm{E}-06$ \\
110 & 55 & $3.00 \mathrm{E}-06$ \\
100 & 50 & $5.00 \mathrm{E}-07$ \\
95 & 47.5 & $3.00 \mathrm{E}-07$ \\
90 & 45 & $2.00 \mathrm{E}-07$ \\
85 & 42.5 & $1.00 \mathrm{E}-07$ \\
80 & 40 & $6.00 \mathrm{E}-08$ \\
\hline
\end{tabular}


Table 5.6 Experimental results of COD propagation rate for frequency $f=10 \mathrm{~Hz}$ under constant load ratio $R=0.5$ and sinusoidal waveform

\begin{tabular}{ccc}
\hline$P_{\max }(\mathrm{lb})$. & $P_{\min }(\mathrm{lb})$. & $d C O D / d N($ in./cycle $)$ \\
130 & 65 & $1.00 \mathrm{E}-05$ \\
120 & 60 & $3.00 \mathrm{E}-06$ \\
110 & 55 & $1.00 \mathrm{E}-06$ \\
100 & 50 & $3.00 \mathrm{E}-07$ \\
90 & 45 & $1.00 \mathrm{E}-07$ \\
80 & 40 & $3.00 \mathrm{E}-08$ \\
\hline
\end{tabular}

\subsubsection{Crack growth rate determination by compliance method}

The accuracy of crack propagation rate $d a / d N$ depends on the measurement of crack length, which is a formidable problem confronted by researchers of fracture mechanics. The crack opening displacement (COD) is much easier to measure than the crack length for the CDCB specimen. The difficulty of measuring crack propagation rate is avoided by recording COD propagation rate. The transformation from crack opening displacement propagation rate $d C O D / d N$ to crack propagation rate $d a / d N$ is given as

$$
\frac{d a}{d N}=\frac{d a}{d C} \frac{d C}{d N}=\frac{1}{k P} \frac{d C O D}{d N}
$$


where $N$ is the number of cycles, $P$ is the applied load, and $k$ is a constant that is equal to the $d C / d a$ value of the $\mathrm{CDCB}$ specimen. Tables 5.7, 5.8, and 5.9 show the crack propagation rate $d a / d N$ obtained from experimental data of $d C O D / d N$ by using Eq. (5.10).

Table 5.7 The crack propagation rate $d a / d N$ for frequency $f=0.1 \mathrm{~Hz}$

\begin{tabular}{ccccc}
\hline$G_{\max }(\mathrm{lb} . / \mathrm{in})$. & $G_{\min }(\mathrm{lb} . / \mathrm{in})$. & $\Delta G(\mathrm{lb} . / \mathrm{in})$. & $d C O D / d N($ in./cycle) & $d a / d N($ in./cycle $)$ \\
\hline 2.00 & 0.50 & 1.50 & $5.00 \mathrm{E}-05$ & $1.30 \mathrm{E}-03$ \\
1.70 & 0.43 & 1.28 & $1.00 \mathrm{E}-05$ & $2.82 \mathrm{E}-04$ \\
1.43 & 0.36 & 1.07 & $5.00 \mathrm{E}-06$ & $1.54 \mathrm{E}-04$ \\
1.18 & 0.30 & 0.89 & $9.00 \mathrm{E}-07$ & $3.04 \mathrm{E}-05$ \\
0.96 & 0.24 & 0.72 & $3.00 \mathrm{E}-07$ & $1.13 \mathrm{E}-05$ \\
0.76 & 0.19 & 0.57 & $1.00 \mathrm{E}-07$ & $4.22 \mathrm{E}-06$ \\
\hline
\end{tabular}

Table 5.8 The crack propagation rate $d a / d N$ for frequency $f=1 \mathrm{~Hz}$

\begin{tabular}{ccccc}
\hline$G_{\max }$ (lb./in.) & $G_{\min }(\mathrm{lb} . / \mathrm{in})$. & $\Delta G(\mathrm{lb} . / \mathrm{in})$. & $d C O D / d N($ in./cycle $)$ & $d a / d N($ in./cycle $)$ \\
\hline 2.00 & 0.50 & 1.50 & $3.00 \mathrm{E}-05$ & $7.80 \mathrm{E}-04$ \\
1.70 & 0.43 & 1.28 & $5.00 \mathrm{E}-06$ & $1.41 \mathrm{E}-04$ \\
1.43 & 0.36 & 1.07 & $3.00 \mathrm{E}-06$ & $9.22 \mathrm{E}-05$ \\
1.18 & 0.30 & 0.89 & $5.00 \mathrm{E}-07$ & $1.69 \mathrm{E}-05$ \\
1.07 & 0.27 & 0.80 & $3.00 \mathrm{E}-07$ & $1.07 \mathrm{E}-05$ \\
0.96 & 0.24 & 0.72 & $2.00 \mathrm{E}-07$ & $7.51 \mathrm{E}-06$ \\
0.86 & 0.21 & 0.64 & $1.00 \mathrm{E}-07$ & $3.98 \mathrm{E}-06$ \\
0.76 & 0.19 & 0.57 & $6.00 \mathrm{E}-08$ & $2.53 \mathrm{E}-06$ \\
\hline
\end{tabular}


Table 5.9 The crack propagation rate $d a / d N$ for frequency $f=10 \mathrm{~Hz}$

\begin{tabular}{ccccc}
\hline$G_{\max }(\mathrm{lb} . / \mathrm{in})$. & $G_{\min }(\mathrm{lb} . / \mathrm{in})$. & $\Delta G(\mathrm{lb} . / \mathrm{in})$. & $d C O D / d N($ in./cycle) & $d a / d N($ in./cycle $)$ \\
\hline 2.00 & 0.50 & 1.50 & $1.00 \mathrm{E}-05$ & $2.60 \mathrm{E}-04$ \\
1.70 & 0.43 & 1.28 & $3.00 \mathrm{E}-06$ & $8.45 \mathrm{E}-05$ \\
1.43 & 0.36 & 1.07 & $1.00 \mathrm{E}-06$ & $3.07 \mathrm{E}-05$ \\
1.18 & 0.30 & 0.89 & $3.00 \mathrm{E}-07$ & $1.01 \mathrm{E}-05$ \\
0.96 & 0.24 & 0.72 & $1.00 \mathrm{E}-07$ & $3.76 \mathrm{E}-06$ \\
0.76 & 0.19 & 0.57 & $3.00 \mathrm{E}-08$ & $1.27 \mathrm{E}-06$ \\
\hline
\end{tabular}

\subsubsection{Constant strain energy release rate range calculation}

The contoured double cantilever beam (CDCB) specimen was used for evaluating fracture toughness of bonded wood-FRP interfaces under mode-I loading. The strain energy release rate $G$ is given as

$$
G=\frac{P^{2}}{2 b} \frac{d C}{d a}
$$

where, $P=$ the applied load; $b=$ the thickness of the specimen; $C=$ the compliance; and $a$ $=$ the crack length. For one complete cycle (see Fig. 2.7), the maximum and minimum values are

$$
G_{\max }=\frac{P_{\max }^{2}}{2 b} \frac{d C}{d a}
$$




$$
G_{\min }=\frac{P_{\min }^{2}}{2 b} \frac{d C}{d a}
$$

The strain energy release rate range $\Delta G$ is easily obtained from the difference of equations (5.12) and (5.13). For the CDCB specimen, the rate of change of compliance with respect to crack length $d C / d a$ is a constant, and therefore, the constant strain energy release rate range $\Delta G$ is acquired for constant cyclic loading.

\subsubsection{A modified Paris Law model}

The modified Paris Law equation corresponding to the strain energy release rate range $\Delta G$

$$
\frac{d a}{d N}=B(\Delta G)^{m}
$$

is used to fit the data points in Tables 5.7, 5.8 and 5.9. The relationship of crack propagation rate $d a / d N$ versus the strain energy release rate range $\Delta G$ is shown in Figs. 5.14, 5.15 and 5.16. For each frequency $f$, the constants $B$ and $m$ are listed in Table 5.10, which indicates that both $B$ and $m$ decrease as the frequency $f$ increases. However, equation (5.14) is only valid for a specific frequency $f$, and it fails to explain the frequency effect for the entire experimental data. Two more data points for frequencies of 0.1 and $10 \mathrm{~Hz}$ are acquired by using Eq. (5.14) for comparison with the results obtained above; and they are respectively listed in Tables 5.11 and 5.12. 
Table 5.10. Material constants $B$ and $m$ in Eq. (5.14) for three different frequencies $f$

\begin{tabular}{|c|c|c|}
\hline Frequency $f$ & $B$ & $m$ \\
\hline 0.1 & $9 \times 10^{-5}$ & 5.85 \\
\hline 1.0 & $5 \times 10^{-5}$ & 5.77 \\
\hline 10 & $2 \times 10^{-5}$ & 5.44 \\
\hline
\end{tabular}

Table 5.11 The crack propagation rate $d a / d N$ for frequency $f=0.1 \mathrm{~Hz}$

\begin{tabular}{cccc}
\hline$G_{\max }$ (lb./in.) & $G_{\min }$ (lb./in.) & $\Delta G$ (lb./in.) & $d a / d N($ in./cycle) \\
\hline 1.07 & 0.27 & 0.80 & $1.09 \mathrm{E}-05$ \\
0.86 & 0.21 & 0.64 & $1.86 \mathrm{E}-06$ \\
\hline
\end{tabular}

Table 5.12 The crack propagation rate $d a / d N$ for frequency $f=10 \mathrm{~Hz}$

\begin{tabular}{cccc}
\hline$G_{\max }(\mathrm{lb} . / \mathrm{in})$. & $G_{\min }$ (lb./in.) & $\Delta G$ (lb./in.) & $d a / d N($ in./cycle) \\
\hline 1.07 & 0.27 & 0.80 & $1.69 \mathrm{E}-05$ \\
0.86 & 0.21 & 0.64 & $2.62 \mathrm{E}-06$ \\
\hline
\end{tabular}




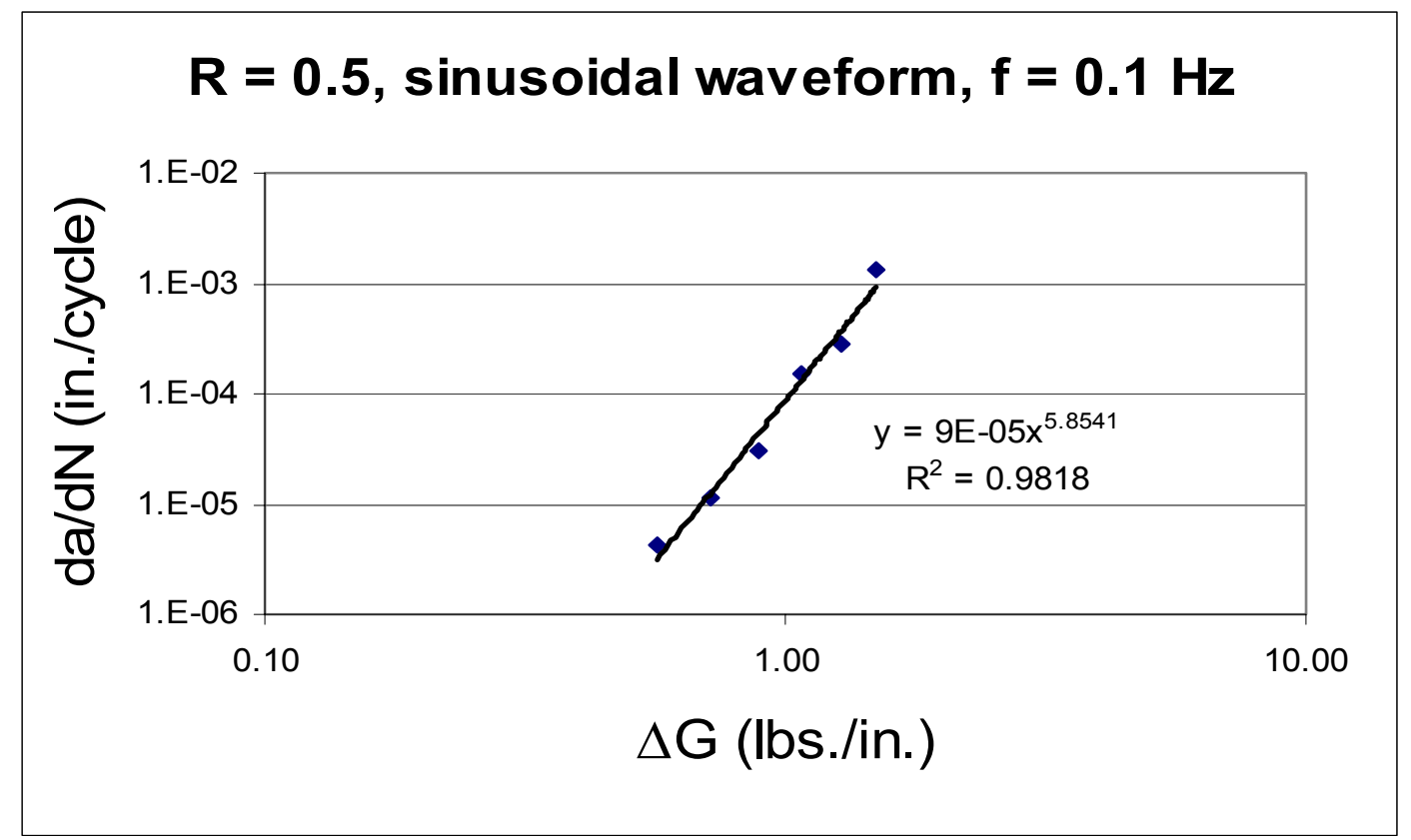

Fig. 5.14 The log-log plot of crack propagation rate $d a / d N$ versus the strain energy release rate range $\Delta G$ for frequency $f=0.1 \mathrm{~Hz}$ 


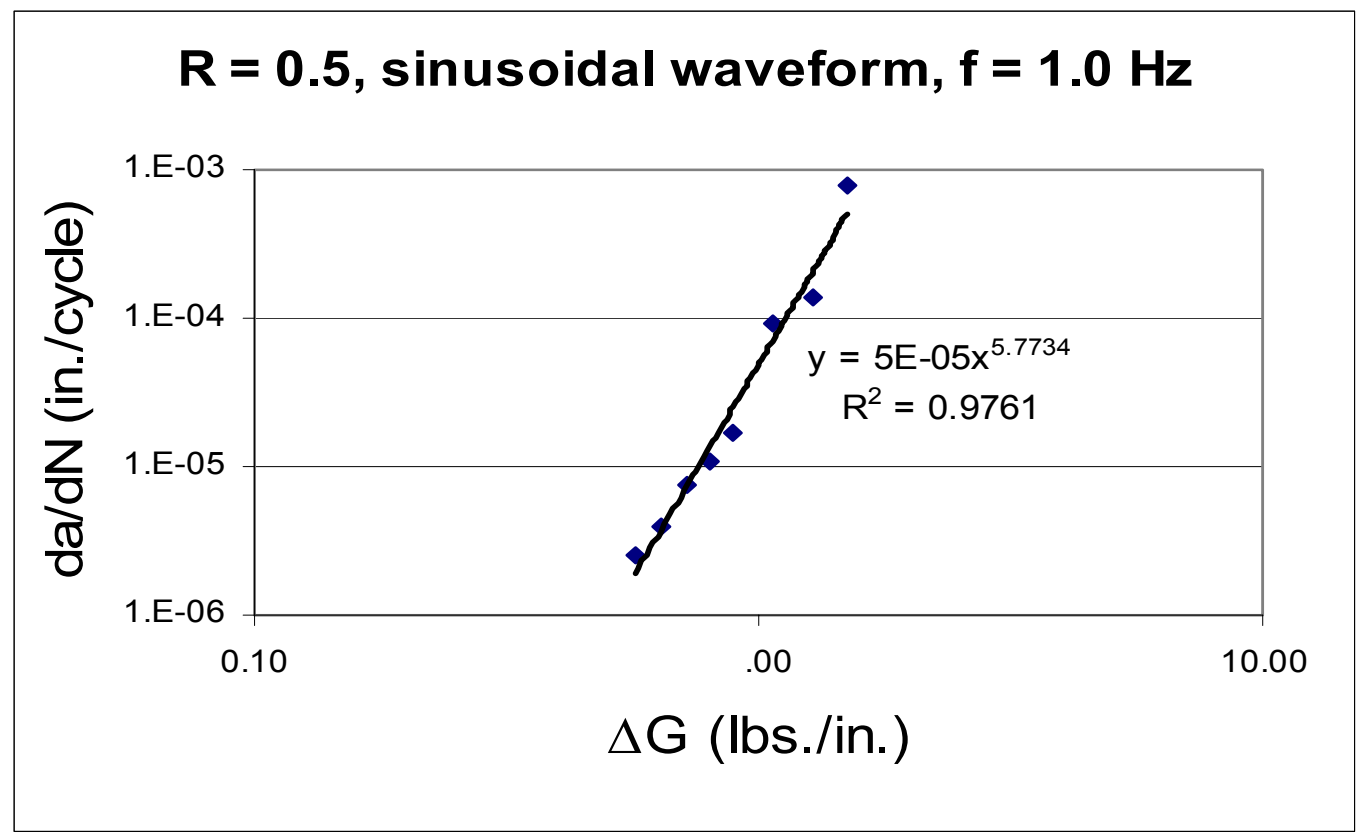

Fig. 5.15 The log-log plot of crack propagation rate $d a / d N$ versus the strain energy release rate range $\Delta G$ for frequency $f=1.0 \mathrm{~Hz}$ 


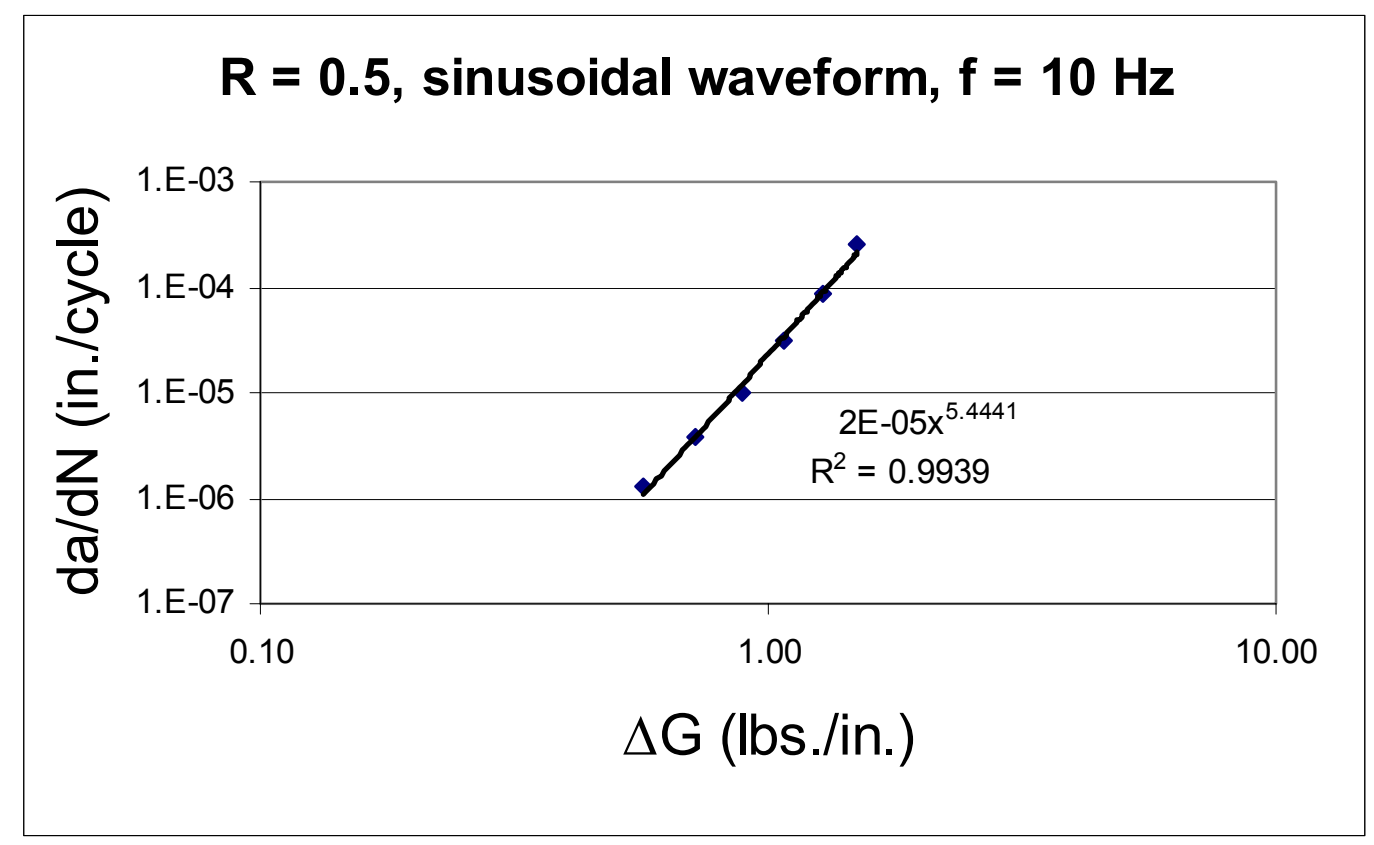

Fig. 5.16 The log-log plot of crack propagation rate $d a / d N$ versus the strain energy release rate range $\Delta G$ for frequency $f=10 \mathrm{~Hz}$ 
Fig. 5.17 shows a double log plot of crack propagation per cycle $d a / d N$ versus strain energy release rate range $\Delta G$, for the frequencies of $0.1,1.0$ and $10 \mathrm{~Hz}$. The results clearly show that frequency has considerable effect on crack growth rate within the strain energy release rate range of 0.5 to $2 \mathrm{lbs}$./in. The crack propagation rate $d a / d N$ increases for the same strain energy release rate range, $\Delta G$, when the loading frequency decreases. The plots of $d a / d t$ versus $\Delta G$ and $d a / d N$ versus $\Delta G_{e q}=\Delta G^{0.07} G_{\text {mean }}^{0.93}$ (see Chapter 3), respectively, are given in Figs. 5.18 and 5.19. These two plots, and especially Fig. 5.18, strongly support the statement that there is appreciable frequency effect on the FRP-wood interface fatigue behavior. At the strain energy release rate range $\Delta G=0.8, d a / d t$ for the loading frequency $f=10 \mathrm{~Hz}$ increases more than one order of magnitude compared with $d a / d t$ for the loading frequency $f=0.1 \mathrm{~Hz}$. From Figs. 5.17 and 5.18, we can see that there is a difference in the definition of the crack propagation rate $d a / d N$ and $d a / d t$, as functions of number of cycles and time, respectively. Therefore, the meaning of crack propagation rate should be clarified for studies of frequency effect. In this Chapter, we define the crack propagation rate dependent on number of cycles in order to keep the same nomenclature as in Chapters 3 and 4 .

There are several models to include frequency effects, one of which believes that crack growth rate is inversely proportional to frequency, such as the following expression proposed by Williams (1977):

$$
\frac{d a}{d N} \propto f^{-\alpha}
$$


where, $\alpha$ is a material constant that generally lies in the range between 0 and 1.0. For example, $\alpha=0.43$ for data obtained from PMMA. Tong and Byrne (1999) also gave a very similar expression with constant $\alpha=0.38$ for a pre-alloyed powder material Udimet 720Li. Considering the above idea and combining with load ratio effect, we believe that the following relationship can define combined frequency and load ratio effects, as

$$
\frac{d a}{d N} \propto f^{-\alpha} \Delta G^{0.07} G_{\text {mean }}^{0.93}
$$

Fig. 5.20 shows data points fitted by linear regression, where the three distinct data lines caused by frequency effects merged approximately into one single function. By trial and error analysis, we obtain $\alpha=0.05$ for phenolic FRP-red maple wood interface under cyclic loading, and we propose a modified Paris Law equation as

$$
\frac{d a}{d N}=1 \times 10^{-4}\left(f^{-0.05} \Delta G^{0.07} G_{\text {mean }}^{0.93}\right)^{5.66}
$$

This model can further be used for other similar studies of interface fracture of dissimilar materials, and we recommend this equation for practical engineering applications. 


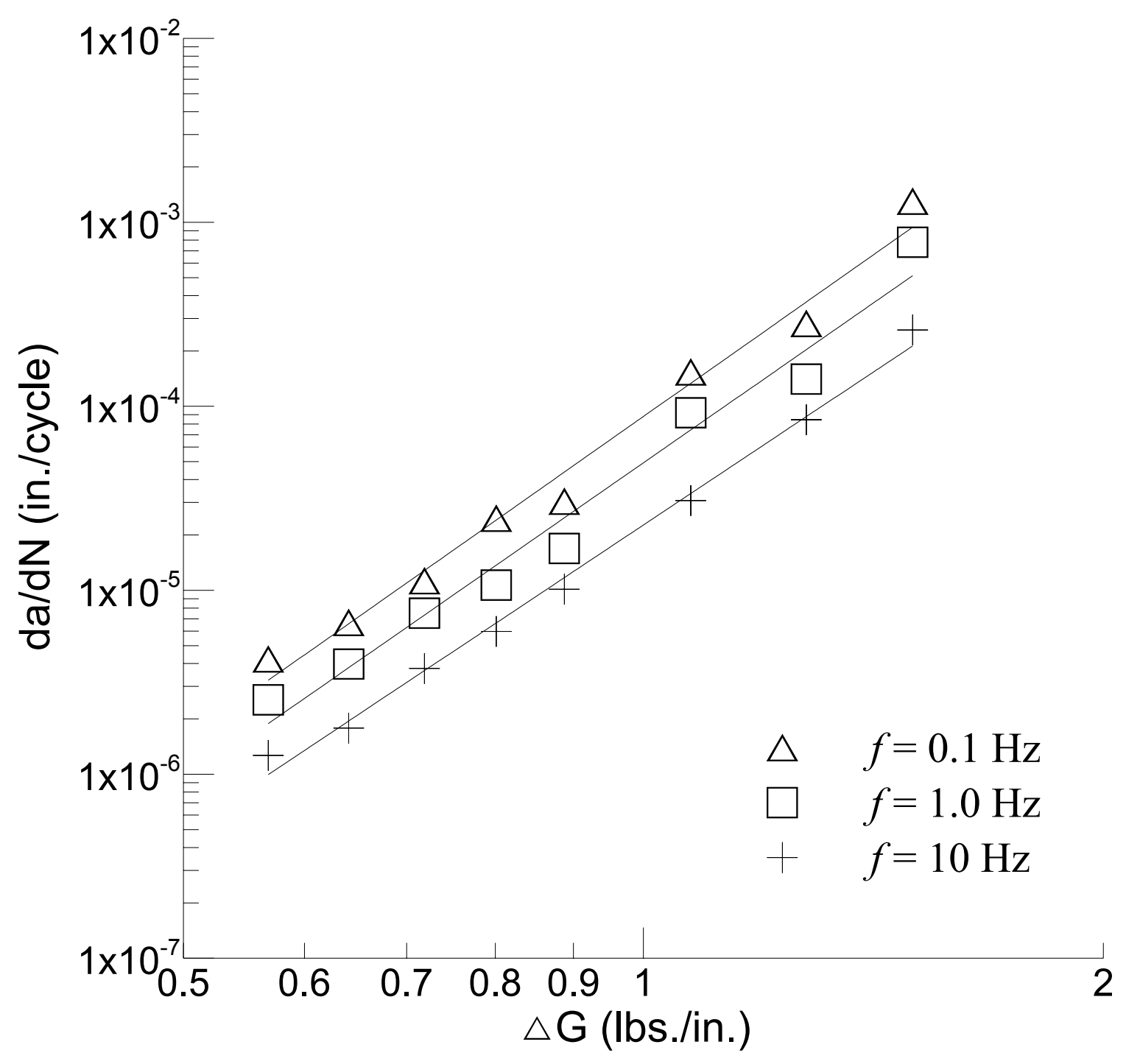

Fig. 5.17 The log-log plot of crack propagation rate $d a / d N$ versus the strain energy release rate range $\Delta G$ for the frequencies of $0.1,1.0$ and $10 \mathrm{~Hz}$ 


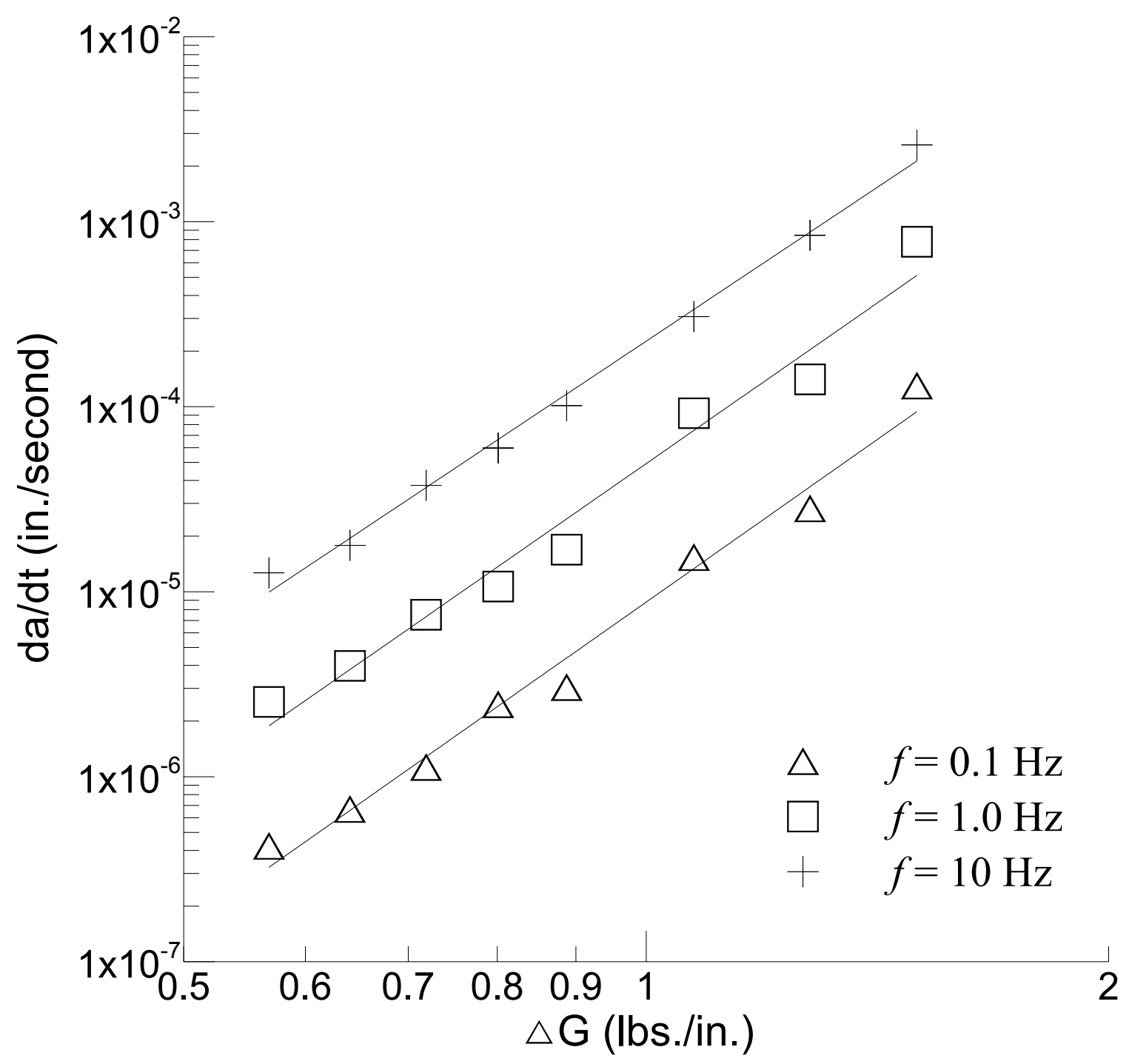

Fig. 5.18 The log-log plot of crack propagation rate $d t / d N$ versus the strain energy release rate range $\Delta G$ for the frequencies of $0.1,1.0$ and $10 \mathrm{~Hz}$ 


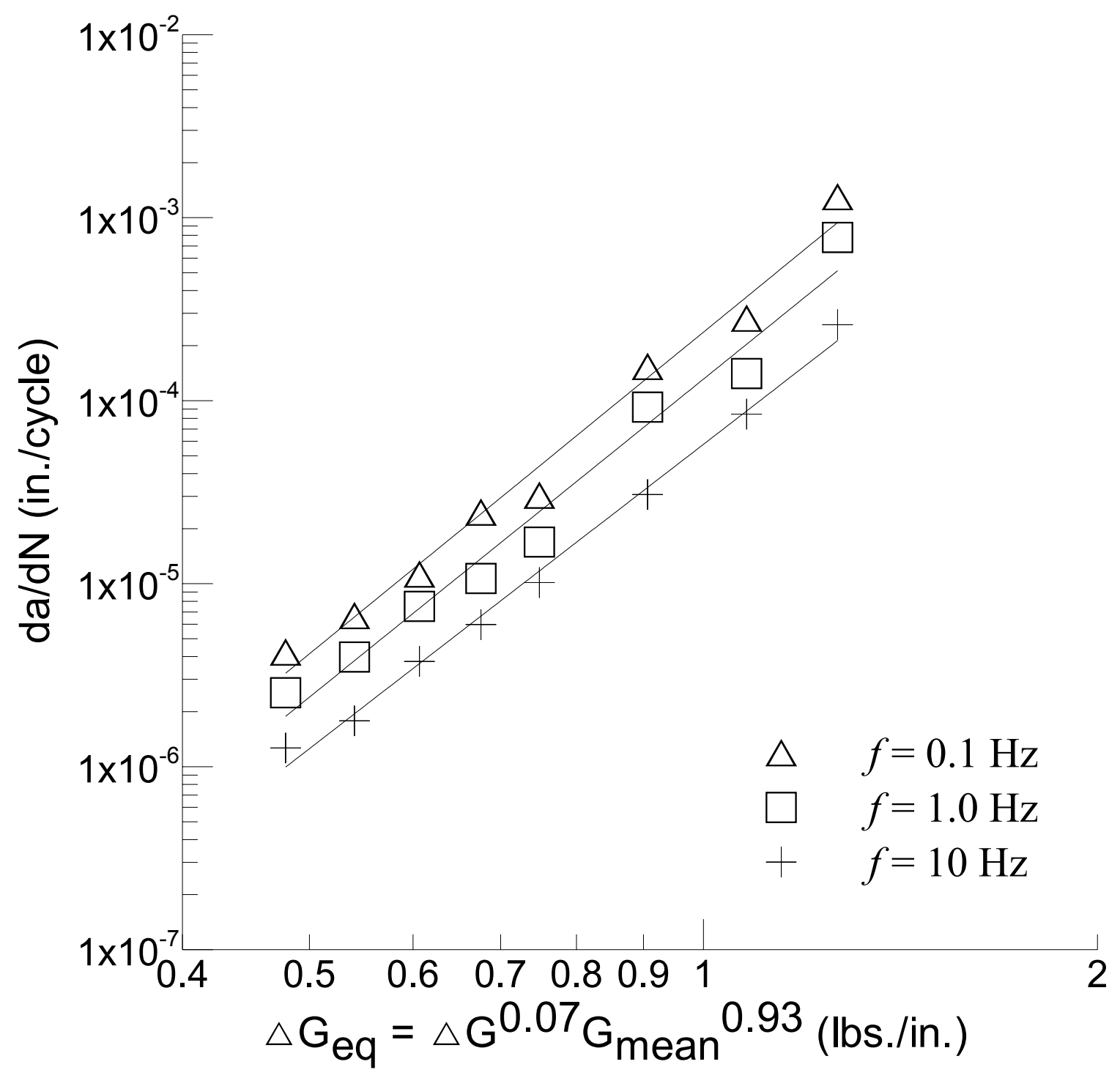

Fig. 5.19 The log-log plot of the crack propagation rate $d a / d N$ versus $\Delta G^{0.07} G_{\text {mean }}{ }^{0.93}$ for the frequencies of $0.1,1.0$ and $10 \mathrm{~Hz}$ 


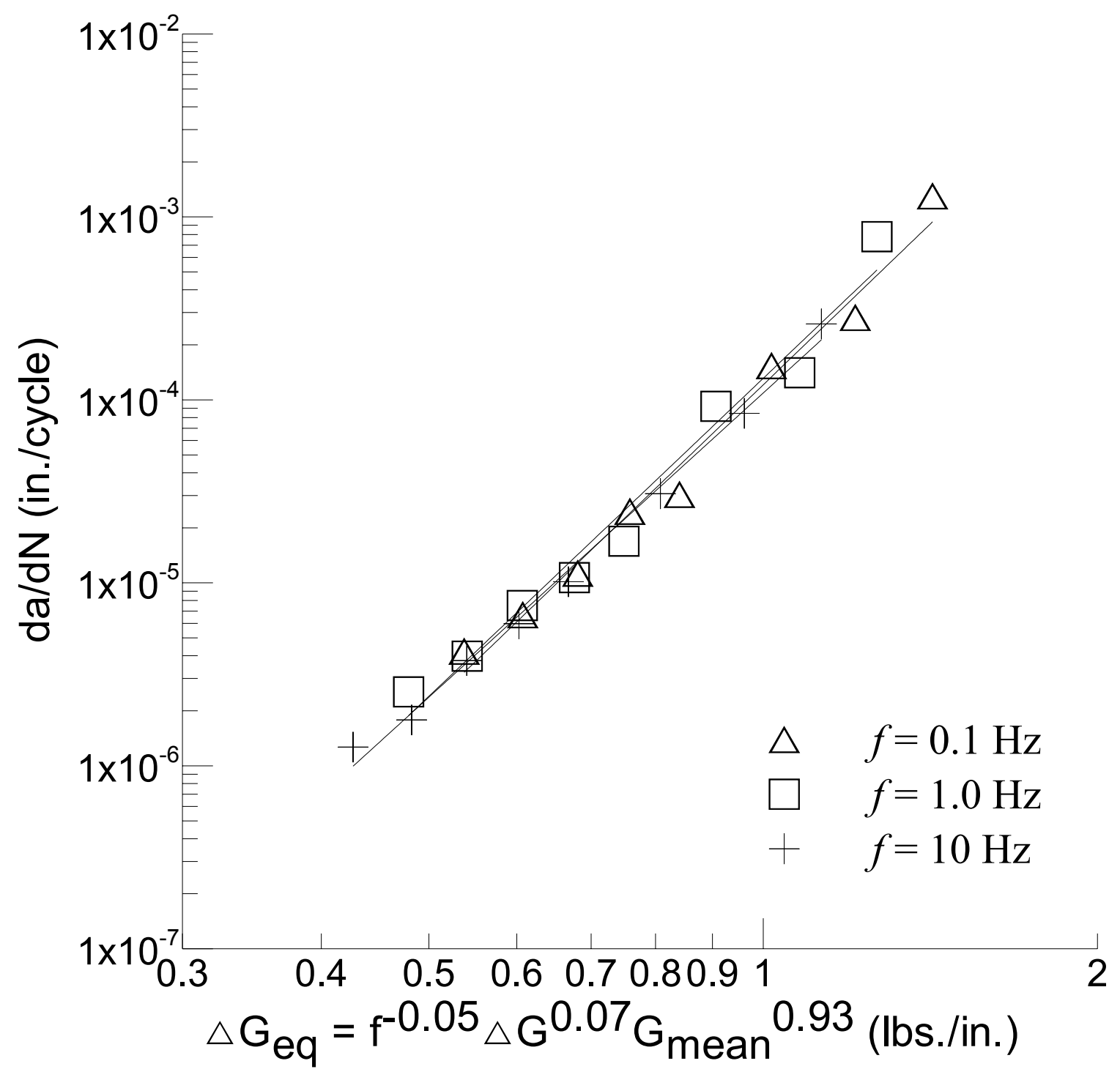

Fig. 5.20 The log-log plot of the crack propagation rate $d a / d N$ versus $f^{-0.05} \Delta G^{0.07} G_{\text {mean }}{ }^{0.93}$ for the frequencies of $0.1,1.0$ and $10 \mathrm{~Hz}$ 


\subsubsection{The failure surface}

A typical photograph of the fracture surface for phenolic FRP-red maple wood CDCB specimen under different frequencies is shown in Fig. 5.21. The fracture surface of the specimens tested showed that interface adhesive failure was predominantly the failure mode of the phenolic FRP-red maple wood. Some of the fractured surfaces happened within the continuous strand mat (CSM) layer of the FRP substrate in combination with interface adhesive failure. For several specimens, substantial fiber bridging was evident during the fracture process. The data having bridging effect was disregarded in order to avoid inconsistencies in the data. Failure around the loading pins and the contoured portion of the CDCB specimens, which occurred in the static tests, did not happen in the fatigue tests. The reason of this is probably that the fatigue loading was much lower than the static loading. Thus we conclude that the interface bond failure was the primary failure mode for the phenolic FRP-red maple wood CDCB specimens under different frequencies. 


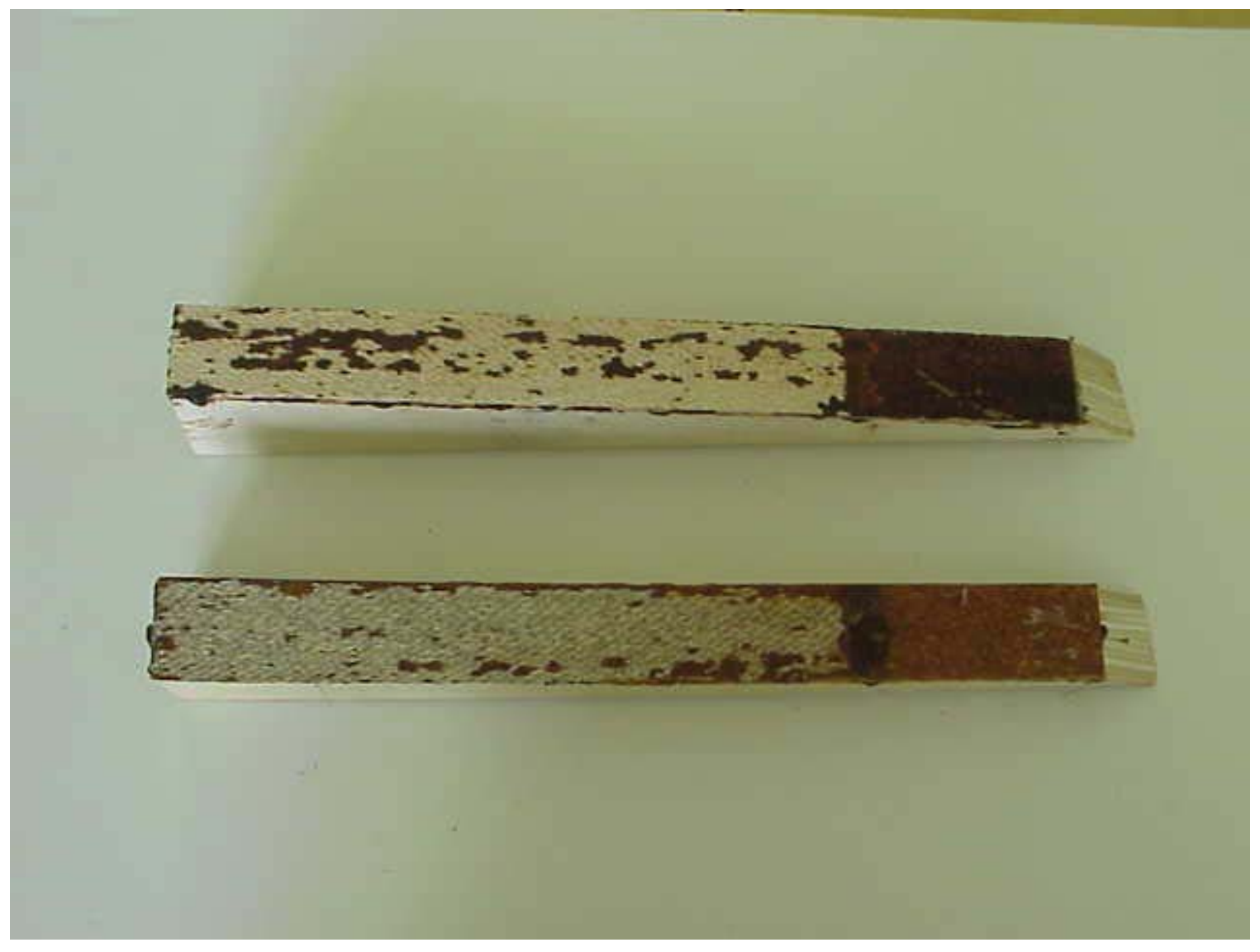

Fig. 5.21 A typical fracture surface for the phenolic FRP-red maple wood CDCB specimen under different frequencies 


\subsection{Conclusions}

The frequency effect on the crack propagation rate of the interface bond between red maple wood and phenolic FRP composite is studied in this chapter. By using a contoured double cantilever beam (CDCB) specimen corresponding to constant strain energy release rate range $\Delta G$, three sets of tests for the frequencies of $0.1,1.0$ and $10 \mathrm{~Hz}$ are performed. All the tests were carried out under constant amplitude cyclic loading at room temperature and laboratory conditions. A modified Paris Law equation is proposed to include both the frequency and load ratio effects. The results of this chapter are summarized as follows:

1. The Paris Law equation using strain energy release rate as a controlling parameter can be efficiently applied to a given value of frequency $f$ :

$$
\frac{d a}{d N}=B(\Delta G)^{m}
$$

where $B$ and $m$ are material constants, which are strongly dependent on frequency $f$. Both $B$ and $m$ decrease as the frequency $f$ increases.

2. The load frequency is an important parameter for crack growth rate of the bonded interface.

3. It is believed that the following relationship is valid 


$$
\frac{d a}{d N} \propto f^{-\alpha} \Delta G^{0.07} G_{\text {mean }}^{0.93}
$$

where $\alpha$ is a material constant determined empirically.

4. The following modified Paris Law equation is proposed for the red maple woodphenolic FRP bonded interface

$$
\frac{d a}{d N}=1 \times 10^{-4}\left(f^{-0.05} \Delta G^{0.07} G_{\text {mean }}^{0.93}\right)^{5.66}
$$

This equation can efficiently represent the combined load ratio and frequency effects for crack propagation rate of wood-FRP interface bond, and it can further be used for other similar studies of interface fracture of dissimilar materials. 


\section{CHAPTER 6}

\section{THRESHOLD}

\subsection{Introduction}

The stress intensity factor range $\Delta K$ at the end of the region I of a fatigue process (see Fig. 1.1) is called the fatigue crack growth threshold, $\Delta K_{t h}$. That is, the fatigue threshold is the lower end of a crack growth rate $d a / d N$ versus stress intensity factor range $\Delta K$ curve. ASTM E-647 (1995) defines the fatigue crack growth threshold, $\Delta K_{t h}$, as an asymptotic value of $\Delta K$ at which the crack growth rate $d a / d N$ approaches zero. An operational definition value of $\Delta K_{t h}$ for most materials is given as that $\Delta K$ which corresponds to a fatigue crack growth rate of $10^{-10} \mathrm{~m} /$ cycle. The fatigue crack growth threshold, $\Delta K_{t h}$, is a highly important parameter for many practical purposes, since the inherent crack or flaw of materials will propagate in a very slow rate (generally assumed to be zero growth rate) when the applied stress intensity factor range is below the value of the fatigue crack growth threshold, $\Delta K_{t h}$. The threshold itself can be considered the dividing line between the propagation and non-propagation of a fatigue crack. A better understanding of threshold fatigue crack behavior would increase the confidence of design engineers to optimize structures in term of cost, performance, and safety. As pointed out by Marci (1992) a fatigue crack growth threshold is a material parameter useful for material characterization and fatigue crack growth design under all fatigue 
loading conditions. Next, a summary of the tests methods on determination of the fatigue threshold, either standardized tests or widely used by researchers, is given, to provide an insight of currently used methods for fatigue threshold determination.

The most common and widely used method to determine the fatigue threshold is the load shedding technique. In this method, a standard compact tension (CT) or middletension (MT) specimen has been acceptably pre-cracked and cycled at $\Delta K$ values corresponding to the crack growth within the Paris Law regime. ASTM E-647 (1995) suggests initiating fatigue cracks at the lowest stress intensity factor possible and the precracking growth rate of less than $10^{-8} \mathrm{~m} /$ cycle. This procedure is started by cycling at $\Delta K$ and $K_{\max }$ levels equal to or greater than the terminal precracking values. The load is decreased gradually as the crack grows. This step is repeated until the threshold, $\Delta K_{t h}$, for no crack growth or crack growth rate smaller than $10^{-10} \mathrm{~m} / \mathrm{cycle}$ is reached. The cyclic frequency and load ratio $R$ are held constant during the test. That is why it is sometimes called constant $R$ test method. Load shedding test may be conducted at decreasing loads in stages at selected crack length intervals. With the computer controlled servo/electro hydraulic test machine available, load shedding test can be carried out continuously by an automated technique without human intervention (for example, with the use of an analog or digital computer).

As Suresh (1998) pointed out, there are some alternatives to the conventional load shedding technique. One is that the specimen is subjected to a cyclic tensile load at stress intensity levels well below the anticipated fatigue threshold value; raise the load by a 
small percentage if no crack growth is detected. Repeat this procedure until the threshold for the onset of crack growth is reached. The other one is increasing load ratio $R$ to measure an intrinsic fatigue threshold. The basic idea of this method is to hold $K_{\max }$ fixed and reduce $\Delta K$ progressively by raising load ratio $R$; this method is known as the constant $K_{\max }$ test method. Constant $K_{\max }$ test method has been used to produce closure-free threshold conditions for threshold experiments. As pointed out by Suresh (1998), this method failed to explain the load ratio effect on the near threshold crack growth rate. Another testing method, the constant mean $K_{I}$ test method, is mentioned and used by Marci (1992). Basically, the mean value of the stress intensity factor is kept the same in this constant mean $K_{I}$ test method.

In this chapter, the modified load shedding technique (ASTM E-647 1995) is used to determine the fatigue threshold and the results for fatigue crack growth threshold, $\Delta G_{t h}$ of the phenolic FRP-red maple wood bonded interfaces are given. Many studies have shown that the load ratio is a very important parameter influencing the fatigue threshold. A detailed literature review of the load ratio effect on the fatigue threshold is given. For the loading frequency, especially at low frequency, it has not been observed significant effects on the fatigue threshold (see Schmidt and Paris 1973 and Ritchie et al. 1999). The loading waveform seems not important to the fatigue threshold at all. Therefore, only the load ratio effect on the fatigue threshold is studied in this investigation. This chapter contains: (1) Literature review, (2) The modified load shedding technique, (3) Fatigue test, (4) Results including determination of the fatigue threshold, $\Delta G_{t h}$ and the load ratio effect on the fatigue threshold for the bonded interfaces, and (5) Conclusions. 


\subsection{Literature Review}

The evaluation of fatigue crack growth rate in terms of fracture toughness has been well established since the 1960's. The relationship between the crack growth rate $d a / d N$ and the stress intensity factor range $\Delta K$ has been viewed as a power law in the intermediate region of a fatigue process. However, a rationale for low crack growth rate behavior (threshold region) is lacking. Compared with the behavior of the intermediate region, the threshold region is associated with an increased sensitivity of loading history, load ratio and micro-structural variation, and other factors. The load ratio appears to be of particular importance in establishing a mechanism for the fatigue threshold behavior. In the intermediate region, the crack growth rate increases as the load ratio increases (see Chapter 3). This increase becomes more evident and even larger when the stress intensity factor range $\Delta K$ is approaching to the fatigue threshold $\Delta K_{t h}$. The degree of sensitivity of $\Delta K_{t h}$ to different load ratios $R$ varies from material to material. This variation between materials, unfortunately, could not been distinguished by most empirical laws proposed to simulate the fatigue threshold behavior. Klesnil and Lukas in 1972 put forward a relationship of threshold described with a power law form. This relationship can be expressed as

$$
\Delta K_{t h}=\Delta K_{t h 0}(1-R)^{\gamma}
$$

where $\Delta K_{\text {tho }}$ is the threshold value for the load ratio $R=0$. The load ratio $R$ sensitivity is governed by a material parameter $\gamma$. This relationship (see Eq. 6.1) provides a convenient 
tool of defining the fatigue threshold for material comparisons. After Klesnil and Lukas's work, researchers have applied Eq. (6.1) to various materials and built an intense database for the material parameter $\gamma$. Eq. (6.1) is also applied this project, and the material parameter $\gamma$ would be determined for the phenolic FRP-red maple wood bonded interface for the first time.

Atodaria et al. (1997) studied the load ratio effect on the fatigue threshold intensity of a randomly oriented short carbon fiber reinforced polyether ether ketone (PEEK) composite under tension-tension cyclic loading. The threshold was identified as the value of $\Delta K$ at which the crack growth rate was of the order of $10^{-10} \mathrm{~m} /$ cycle. The load shedding technique as per ASTM Standard E-647 was used to determine the fatigue threshold. The average value from three to four specimens for each load ratio was taken as the representative of the fatigue threshold. They found that the fatigue threshold stress intensity factor for the PEEK composite decreased linearly with increasing load ratio.

Branco, Radon, and Culver (1975b) studied the influence of the mean stress intensity factor $K_{m}$ on the threshold of stress intensity factor range value $\Delta K_{t h}$ for an aluminum alloy. Fatigue tests were conducted at frequency of $0.15 \mathrm{~Hz}$ with a contoured double cantilever beam (CDCB) specimen. They established the threshold value $\Delta K_{\text {th }}$ as $\Delta K$ at the crack speeds around $2.5 \times 10^{-10} \mathrm{~m} /$ cycle $\left(10^{-8} \mathrm{in} /\right.$ cycle $)$. They established the value of $\Delta K_{t h}$ directly from the sigmoidal curves of $d a / d N$ versus $\Delta K$, which was extrapolated to the value $10^{-8} \mathrm{in} /$ cycle. A threshold value of $5000 \mathrm{lb} / \mathrm{in}^{3 / 2}$ was taken for load ratio $R=0$. They found that the threshold of stress intensity factor range value $\Delta K_{\text {th }}$ 
was dependent on mean stress intensity factor $K_{m}$, and $\Delta K_{t h}$ decreased linearly with increasing values of $K_{m}$ larger than $2500 \mathrm{lb} / \mathrm{in}^{3 / 2}(\mathrm{R} \geq 0)$. Although there was a nonlinear relationship, the threshold value $\Delta K_{t h}$ was found to decrease with increasing $K_{m}$.

Schmidt and Paris (1973) investigated the effects of frequency and load ratio on the threshold of stress intensity factor range $\Delta K_{t h}$. The compact tension specimen of 2024-T3 aluminum was used throughout the testing. High frequency tests (considered to be greater than $200 \mathrm{~Hz}$ ) from $342 \mathrm{~Hz}$ to $1000 \mathrm{~Hz}$ were conducted under displacement control, since this would produce a progressive decrease in stress intensity factor with crack extension. They found that the threshold of stress intensity factor range $\Delta K_{t h}$ was decreased with increasing frequencies; however, previous investigations reported that frequencies from 4 to $200 \mathrm{~Hz}$ had no significant effect on $\Delta K_{t h}$. This finding by Schmidt and Paris (1973) is unexpected from the point of view that higher frequencies would exacerbate fatigue crack growth. They tried to explain this unexpected result by heat increase at the crack tip, since the increase of temperature might decrease the threshold. For the higher frequency case, heat might be generated by crack tip plasticity and cause a local temperature rise around the crack tip. For the lower frequency case, heat could be dissipated before a crack would proceed rapidly enough, which would avoid local temperature change near the crack tip. For load ratio $R$ effect, two groups of data for frequencies equal to 300 and $580 \mathrm{~Hz}$ were presented. The threshold of stress intensity factor range $\Delta K_{t h}$ was defined as a $\Delta K$ value with a growth rate less than $2 \times 10^{-8}$ in/cycle. The threshold of stress intensity factor range $\Delta K_{t h}$ tended to decrease with increasing load 
ratios. This was explained on the basis of crack closure phenomena, in which a crack might not be completely open for all portions of the tensile loading.

Nakai, Tanaka and Nakanishi (1981) studied the effects of stress ratio and grain size on near-threshold fatigue crack propagation in low carbon steel. The load ratios were $-1,0,0.25$ and 0.5 with a frequency of $30 \mathrm{~Hz}$. The low rate region denoted as $A$ was divided into two regions $A 1$ and $A 2$ in their relation to the crack growth rate against the effective stress intensity factor range. The effective range of stress intensity factor is defined based on the crack closure measurement and calculated as

$$
\Delta K_{\text {eff }}=K_{\max }-K_{o p}
$$

where $K_{\max }$ and $K_{o p}$ are the maximum stress intensity factor and the stress intensity factor necessary to open the crack. In regions $A 2$ and $B$, the rate was expressed in a unique power function of the effective stress intensity range without consideration of stress ratio and the grain size. In region $A 1$, the effective threshold stress intensity factor increased linearly with the square root of the grain size. The variation of $\Delta K_{t h}$ with respect to the load ratio $R$ was expressed as

$$
\Delta K_{t h}=\Delta K_{t h 0}(1-R)^{\gamma}
$$

where $\Delta K_{t h 0}$ is a material parameter that can be equated to the threshold value $\Delta K_{t h}$ at the load ratio $R=0$, and $\gamma=0.5$ was obtained from their experimental data. They argued that 
the possible reasons for a high crack-opening stress in region $A$ were that the slip band was blocked by the grain boundary and the crack path was on the crystallographic plane.

Ishiyama et al. (1987) defined a threshold stress intensity factor range $\Delta K_{\text {th }}$ as the point below which the crack extension was less than $10 \mu \mathrm{m}$ after $10^{5}$ cycles of loading. The crack growth was measured optically using a comparator. In the actual experiment, after a crack extension was observed at a given $\Delta K$, the load was lowered stepwise until no crack extension could be observed after loading to $10^{5}$ cycles for constant $R$ conditions. The load ratio effect on the range of threshold stress intensity factor was evaluated experimentally. As load ratio $R$ increased, the threshold of stress intensity factor range decreased; when load ratio $R$ approached to 1, the threshold of stress intensity factor range approached to zero. A power relationship, which is identical to Eq. (6.1), was given as

$$
\Delta K_{t h} / \Delta K_{t h 0}=(1-R)^{A}
$$

where $\Delta K_{\text {th }}$ is the $\Delta K_{\text {th }}$ value for $R=0$, and the constant $A=0.89$ for the fine-grained isotropic nuclear graphite.

Ohta and Sasaki (1977) studied the influence of the stress ratio on the threshold level for fatigue crack propagation in high strength steels. Centrally cracked specimens were fatigued at load ratio ranging from -1 to 0.8 . The amplitude of stress intensity factor, $\Delta K / 2$, was decreased with a constant rate, $|d(\Delta K) / d a|$, of $10 \mathrm{~kg} / \mathrm{mm}^{5 / 2}$. As a 
result, the stress intensity threshold levels for fatigue crack propagation, $\Delta K_{t h}$, were influenced by the stress ratio, $R$. The effect of $R$ was expressed as

$$
\left|\Delta K_{t h} / 2\right|_{R}=(1-R)^{\gamma}\left|\Delta K_{t h} / 2\right|_{R=0}
$$

Where $\gamma=0.71$. This expression coincides with that of Klesnil and Lukas (1972b).

Cooke et al. (1975) studied the slow fatigue crack growth and threshold behavior of quenched and tempered En 24 steel. Fatigue testing was performed at $175 \mathrm{~Hz}$ (air) and $100 \mathrm{~Hz}$ (vacuum). Specimens with single edge notch under three-point bending were tested. In air, constant crack growth rate tests were conducted over a range of rates between $10^{-7} \mathrm{~mm} /$ cycle and $10^{-4} \mathrm{~mm} /$ cycle at three positive load ratios (nominally 0.15 , 0.33 and 0.70 ). In vacuum, tests included $10^{-7} \mathrm{~mm} /$ cycle and $10^{-5} \mathrm{~mm} / \mathrm{cycle}$ at load ratios of $0.17,0.36$ and 0.62 . The apparent threshold for crack growth was estimated independently at a number of load ratio values in each environment. This was done by reducing the load, until no crack growth was detected at $5 \times 10^{6}$ cycles. This condition corresponded to less than $10^{-8} \mathrm{~mm} /$ cycle for air tests and $3 \times 10^{-8} \mathrm{~mm} /$ cycle for vacuum tests. Evaluation of in-air data in terms of $K_{\max }$ and comparison with the $\Delta K$ data showed that $K_{\max }$ became more important as the rate controlling parameter near the threshold. Eq. (6.1) from Klesnil and Lukas (1972b) was used to fit experimental data and the value of $\gamma$ was obtained to be equal to 0.53 . The behavior controlled by $K_{\max }$ was noted up to a load ratio of 0.37 , above which threshold values tended to be control by $\Delta K$. Their observations supported the occurrence of crack closure effect, which they suggested may 
be related to the effects of load ratio at low growth rate. The tests in vacuum for crack growth rate indicated lack of a systematic effect of load ratio both at low growth rates and also on $\Delta K_{t h}$. The value of $\gamma$ in Eq. (6.1) was equal to 0 in vacuum conditions.

\subsection{The Modified Load Shedding Procedure}

Following the ASTM E-647 (1995) standard, a modified load shedding procedure for determination of the fatigue crack growth threshold, $\Delta G_{t h}$, is presented in this section. This test method involves constant cyclic loading of a contoured double cantilever beam (CDCB) specimen, which has been described in previous Chapters. Crack opening displacement is measured as a function of elapsed fatigue cycles. Crack growth rate is established by a compliance method (see Section 3.4.2) from the crack opening displacement growth rate data. Crack growth rates are expressed as a function of the strain energy release rate range $\Delta G$, which is calculated from expressions based on linear elastic fracture analysis (see Eqs. 2.2, 2.3 and 2.4). The modified load shedding procedure is described as the following five steps (note: there is no absolutely strict order):

1. Define the threshold strain energy release rate range $\Delta G_{t h}$ as the strain energy release rate range at which the crack growth rate $d a / d N$ is less than $3 \times 10^{-8} \mathrm{in} /$ cycle $\left(7.62 \times 10^{-10} \mathrm{~m} /\right.$ cycle $)$, which is hypothetically assigned as the near threshold value of crack propagation rate. 
2. Estimate the cyclic load corresponding to the threshold strain energy release rate range $\Delta G_{t h}$ for the given value of load ratio $R$.

3. Decrease the load gradually as the crack grows. If the crack growth rate $d a / d N$ is greater than $10^{-8} \mathrm{~m} /$ cycle, decrease the load by about $5 \%$ of the previous load level. If the crack growth rate $d a / d N$ is less than $10^{-8}$ $\mathrm{m} /$ cycle, decrease the load by about $2 \%$ of the previous load level.

4. Record the test data until no pronounced crack growth could be observed and the crack growth rate $d a / d N$ of less than $3 \times 10^{-8}$ in/cycle $\left(7.62 \times 10^{-10}\right.$ $\mathrm{m} /$ cycle) is achieved.

5. The load ratio $R$ remains constant during the entire test to avoid any stress history effect.

A schematic illustration of load shedding during the modified $G$-decreasing test is shown in Fig. 6.1. Even though the parameter of normalized $K$-gradient, $C=1 / K \times$ $d K / d a$, is not used in this test procedure, the requirement for the rate of load shedding to be gradual enough is met by controlling the reduction of cyclic load. First, the reduction in $P_{\max }$ of sequential load steps did not exceed $10 \%$ of the previous $P_{\max }$. Second, when the crack growth rate was less than $10^{-8} \mathrm{~m} / \mathrm{cycle}$, the condition for continuous load shedding of $\left(P_{\max 1}-P_{\max 2}\right) / P_{\max 1} \leq 0.02$ was satisfied. 


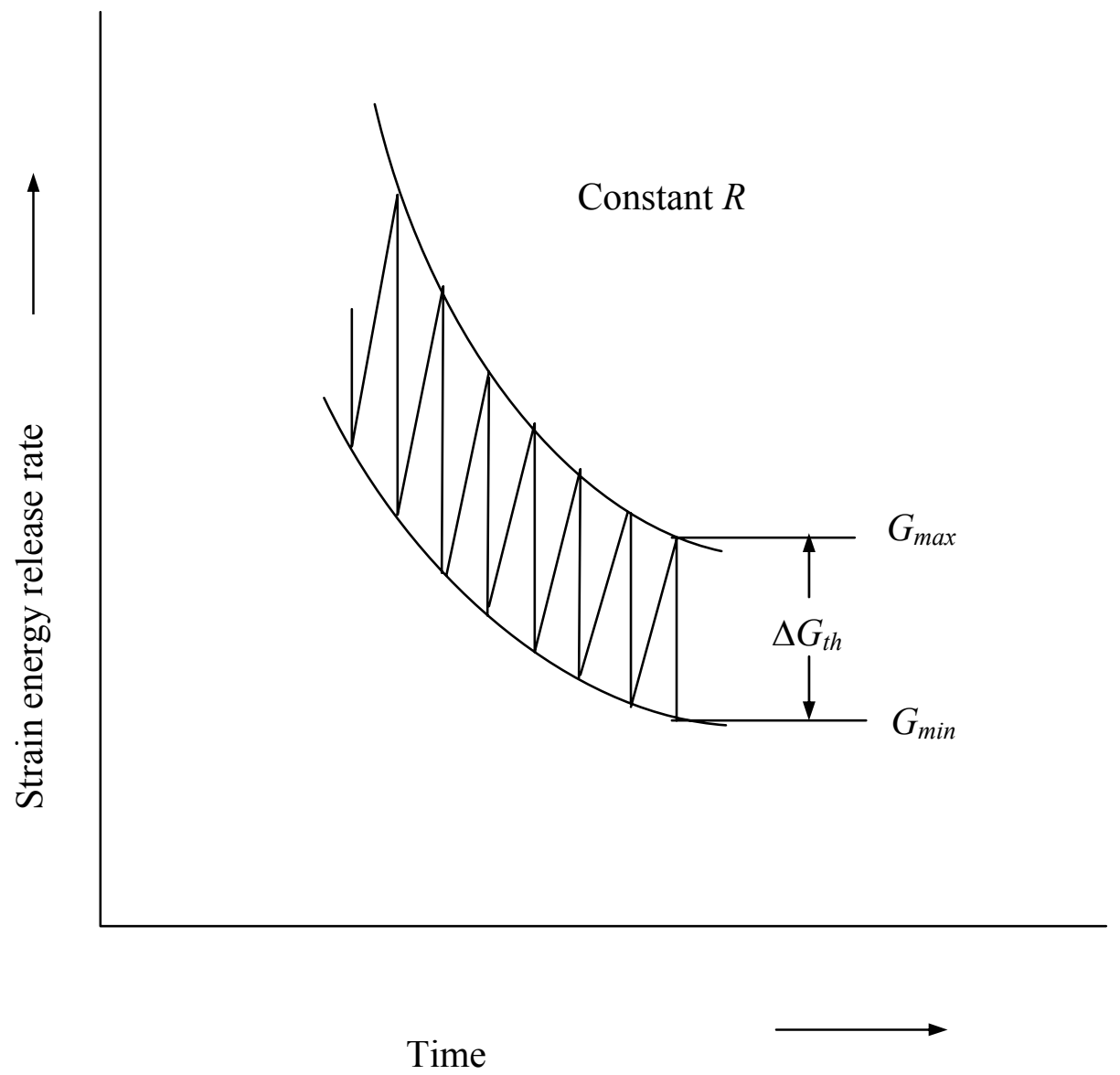

Fig. 6.1 A typical $G$ decreasing test by stepped load shedding 


\subsection{Fatigue Test}

The fatigue threshold of phenolic FRP-wood interface bond was determined in this chapter. The details of the specimen and materials have been described in previous chapters. As before, fatigue tests were conducted in a load control mode. The crack opening displacement growth rate was transformed to crack growth rate by the compliance method (see section 2.6). The modified load shedding procedure in section 6.3 was followed to determine the fatigue threshold. Three groups of tests for load ratios $R$ equal to $0.1,0.3$ and 0.5 were carried out to study the influence of the load ratio on the fatigue threshold of the bonded interface. The sinusoidal waveform and frequency of 1 $\mathrm{Hz}$ were kept the same throughout the whole testing for each group of tests. All fatigue tests were conducted at room temperature in laboratory air on a servo hydraulic MTS testing machine.

\subsection{Results}

This section presents the method used for fatigue threshold, and the load ratio effect on fatigue threshold.

\subsubsection{Determination of the fatigue threshold}

For the phenolic FRP-red maple wood bonded interfaces, the fatigue threshold at the load ratios $R$ equal to $0.1,0.3$ and 0.5 were determined using the modified load 
shedding technique (see section 6.3). The experimental results of strain energy release rate parameters at the fatigue threshold are tabulated in Table 6.1. It is observed that $G_{\max }$ remains relatively constant at the threshold level for different load ratios. Interestingly, if we write $G_{\max }$ as

$$
G_{\max }=G_{\max } \frac{(1-R)}{(1-R)}=\frac{\left(G_{\max }-G_{\min }\right)}{(1-R)}=\frac{\Delta G}{(1-R)}
$$

Then, $\Delta G /(1-R)$ remains relatively constant at the threshold level over the range of load ratios $R$ tested here. The fatigue threshold $\Delta G_{t h}$ is found to decrease with increasing load ratios. The $G_{\max }$ control or dominance may be related to the crack closure effect, which is going to be discussed in the next section.

Table 6.1 Experimental results of strain energy rate parameters at the fatigue threshold

\begin{tabular}{cccc}
\hline$R$ & $G_{\max }(\mathrm{lbs} / \mathrm{in})$. & $G_{\min }(\mathrm{lbs} / \mathrm{in})$. & $\Delta G$ (lbs/in.) \\
\hline 0.1 & 0.339 & 0.003 & 0.336 \\
0.3 & 0.325 & 0.029 & 0.296 \\
0.5 & 0.335 & 0.084 & 0.251 \\
\hline
\end{tabular}




\subsubsection{Load ratio effect on the fatigue threshold}

A substantial crack closure effect has been observed during the present fatigue threshold studies. The dominant role of crack closure on near threshold crack growth and load ratio effect on the fatigue threshold have been recognized and acknowledged by many researchers (Suresh and Ritchie, 1984). Despite the lack of convincing analytical formulations for crack closure, Schmidt and Paris (1973) presented a model to account for the dependence of the threshold stress intensity range $\Delta K_{t h}$ on load ratio $R$ by the crack closure concept. A schematic representation of the Schmidt and Paris's model for the load ratio $R$ dependence of fatigue thresholds is shown in Fig. 6.2. Two distinct regimes of threshold variation with load ratio $R$ arise based on two assumptions: (1) the stress intensity needed to open a crack $K_{c l}$ is constant and independent of load ratio $R$, and (2) the effective threshold stress intensity range $\Delta K_{0}$, which is the portion of the stress intensity range above $K_{c l}$ and necessary to produce crack growth at the threshold, is constant and independent of load ratio $R$.

Regime 1: at low load ratios and $K_{\min } \leq K_{c l}$

$$
\begin{gathered}
K_{\text {max }}=K_{c l}+\Delta K_{0}=\text { const } \\
\Delta K_{t h}=K_{\text {max }}-K_{\text {min }}=K_{\text {max }}(1-R)=\left(K_{c l}+\Delta K_{0}\right)(1-R)
\end{gathered}
$$

Regime 2: at high load ratios and $K_{\min } \geq K_{c l}$ 


$$
\begin{gathered}
\Delta K_{t h}=\Delta K_{0}=\text { const } \\
K_{\max }=\frac{\Delta K_{t h}}{1-R}=\frac{\Delta K_{0}}{1-R}
\end{gathered}
$$

Even though somewhat arbitrarily chosen, there exists a critical load ratio $R_{c l}$, above which crack closure becomes insignificant. The consistency between this model and experimental data has been reported for metallic materials.

In this study, the Schmidt and Paris's model is successfully used to explain the load ratio effect on the fatigue threshold for the red maple wood-phenolic FRP composite bonded interface. The experimental resuls of the fatigue threshold and maximum strain energy release rate, $\Delta G_{t h}$ and $G_{\max }$, respectively, with respect to load ratio $R$ are shown in Figs. 6.3 and 6.4. It can clearly be seen that the data for load ratio below 0.5 falls into the first regime of the Schmidt and Paris's model. The relationship of

$$
\begin{gathered}
\Delta K_{t h}=f(1-R) \\
K_{\max }=\text { const }
\end{gathered}
$$

can be extracted from Figs. 6.3 and 6.4, approximately. It is noted that $G_{\max }$ remains constant over a large range of load ratio. This implies that there is significant crack 
closure at the crack tip of the bonded interfaces. The crack closure is probably induced by the roughness of the interface and plasticity at the crack wake. Fiber bridging may also contribute to the crack retardation. Therefore, the crack closure phenomena probably caused the load ratio effect on the fatigue threshold of the bonded interface. When the load ratio $R$ is less than or equal to 0.5 , the fatigue threshold is $G_{\max }$ controlled, and if the $G_{\max }$ is less than about $0.34 \mathrm{lbs}$./in., the fatigue failure can be ignored for the red maple wood-phenolic FRP composite bonded interface.

The influence of load ratio $R$ on the fatigue threshold has also been investigated extensively using Klesnil and Lukas's model (Eq. 6.1) for metals (see Branco et al. 1975, Nakai et al. 1981, Ohta et al. 1977). As a result, these references reported that the fatigue threshold decreased as the load ratio increased; in some cases, a linear relationship between the fatigue threshold and the load ratio was reported, such as the results published by Atodaria et al. (1997) for a randomly oriented short carbon fiber reinforced PEEK composite. It seems that the phenolic FRP-red maple wood bonded interface also shows a similar behavior. Thus, the Klesnil and Lukas's model is used to fit the experimental data of the red maple wood-phenolic FRP composite bonded interface. The relationship between $\Delta G_{t h}$ and $(1-R)$ is plotted in Fig. 6.5. The parameters of $\Delta G_{t h 0}$ and $\gamma$ in Klesnil and Lukas's model are obtained in this study to be equal to 0.35 and 0.49 , respectively, and the resulting equation is

$$
\Delta G_{t h}=0.35(1-R)^{0.49}
$$




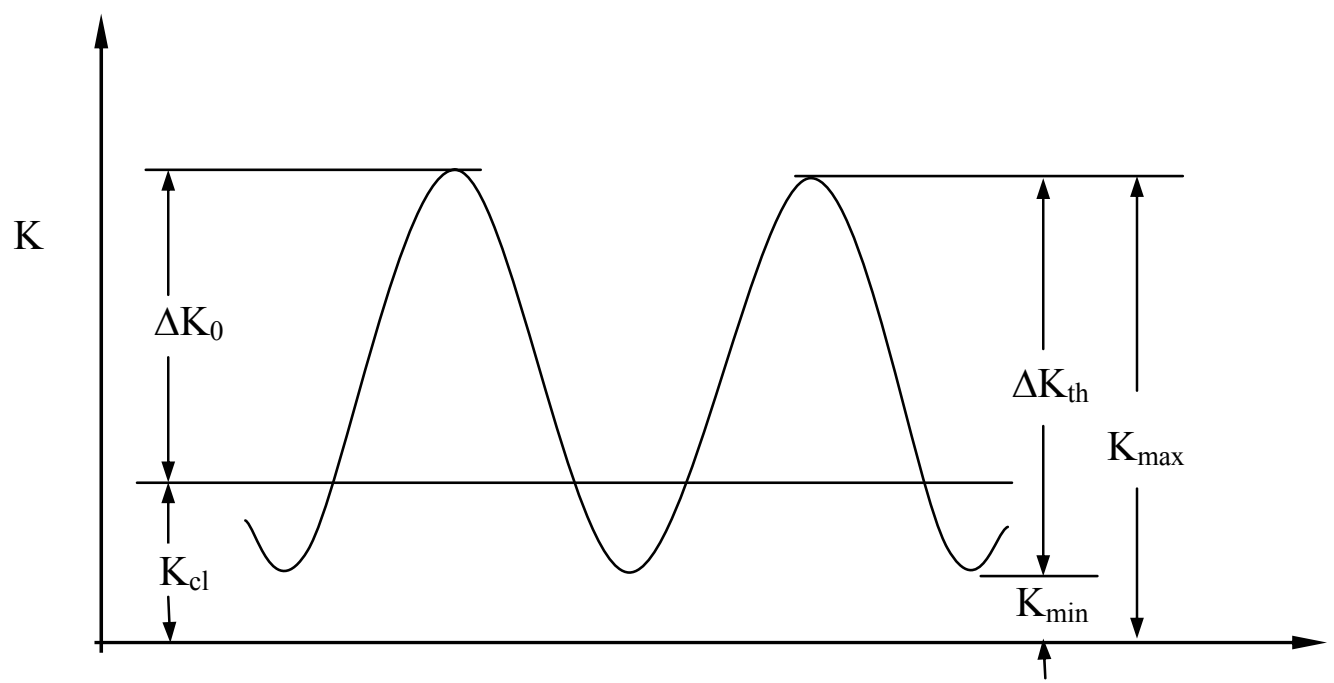

Time

(a) Nomenclature for stress intensity parameters at fatigue threshold

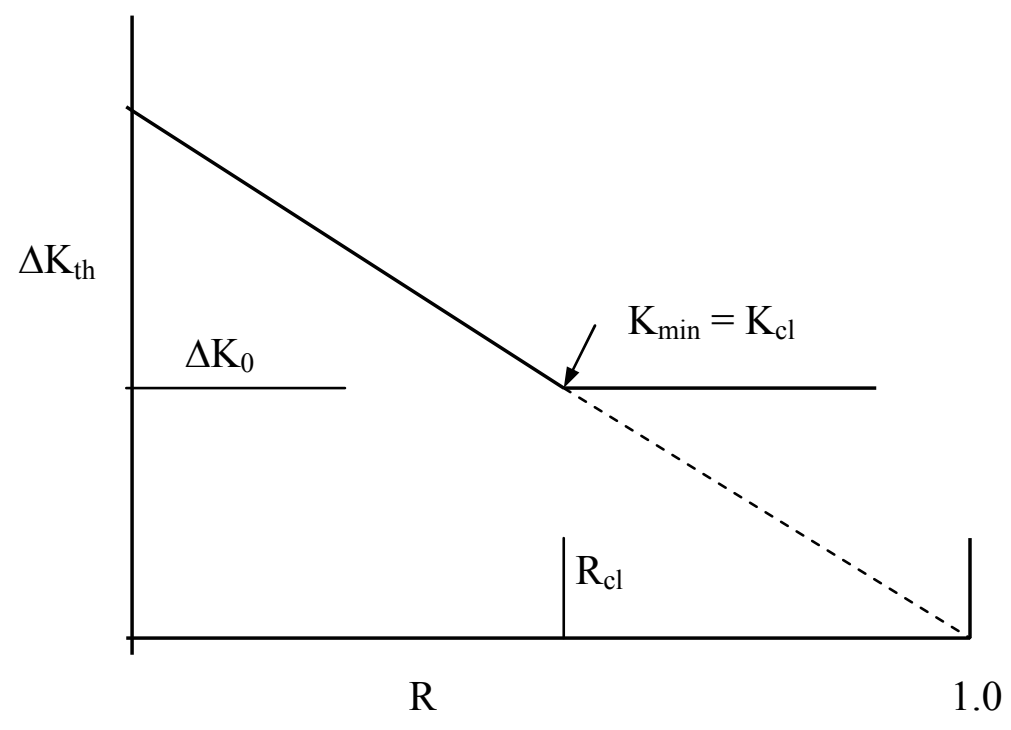

(b) The predicted variation of nominal stress intensity range, $\Delta \mathrm{K}_{\text {th }}$ with load ratio $\mathrm{R}$ 


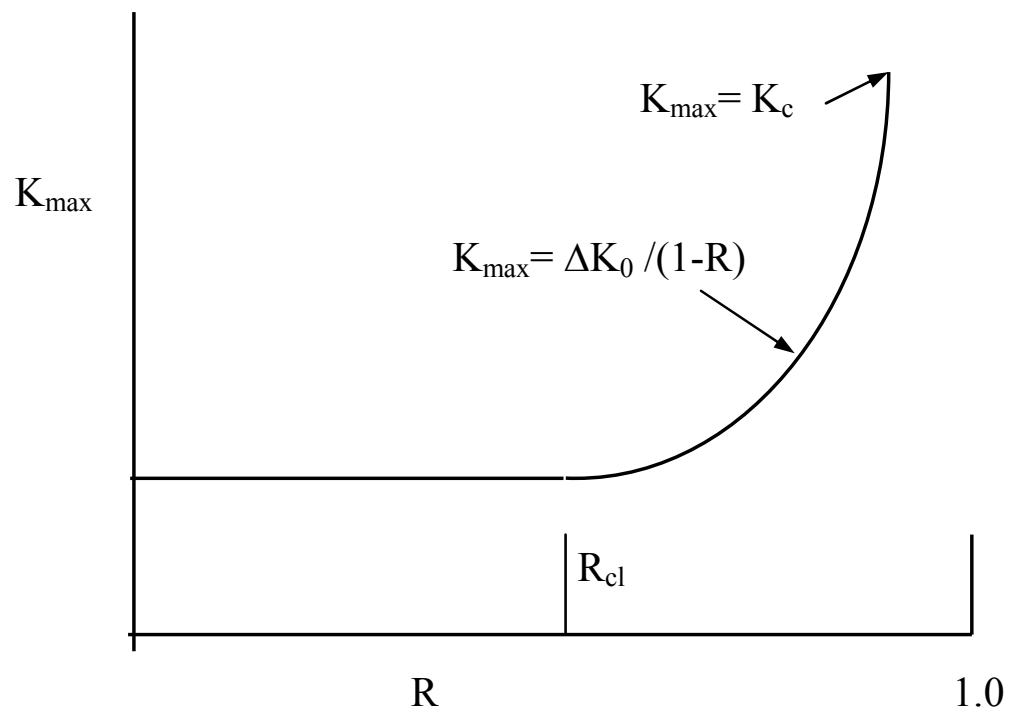

(c) The predicted variation of maximum stress intensity, $\mathrm{K}_{\max }$ at threshold with load ratio $\mathrm{R}$

Figure 6.2 A schematic illustration of Schmidt and Paris's model (1973), where $\mathrm{K}_{\mathrm{cl}}$ and $\mathrm{K}_{\mathrm{c}}$ are the stress intensities at closure and at final failure, respectively, and $R_{c l}$ is the critical load ratio above which crack closure effects are minimal 


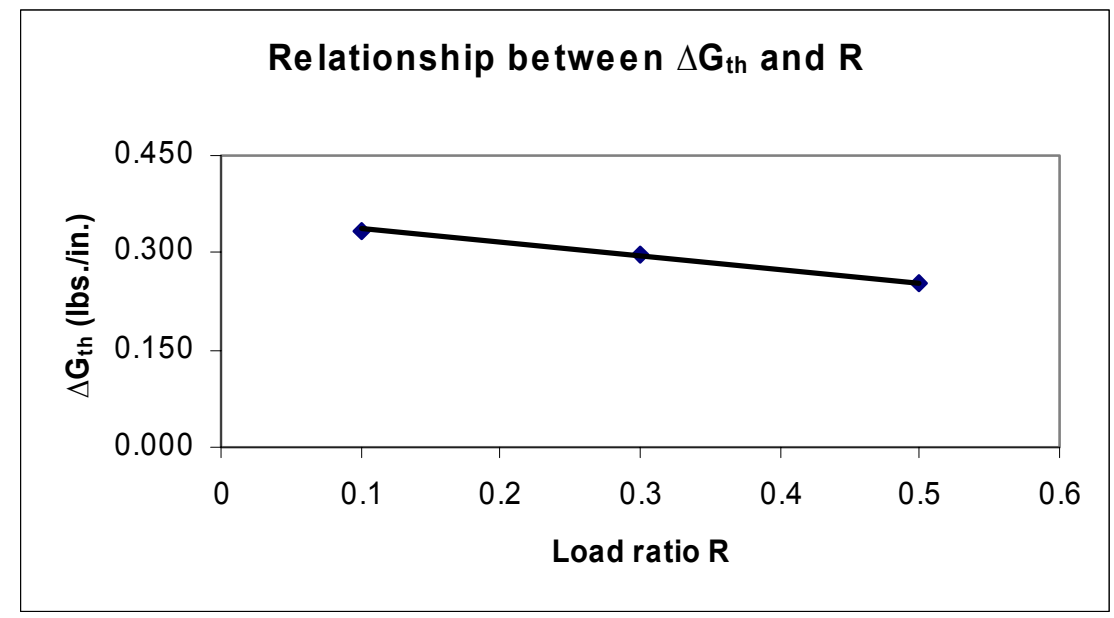

Figure 6.3 Effect of load ratio $R$ on the fatigue threshold $\Delta G_{t h}$

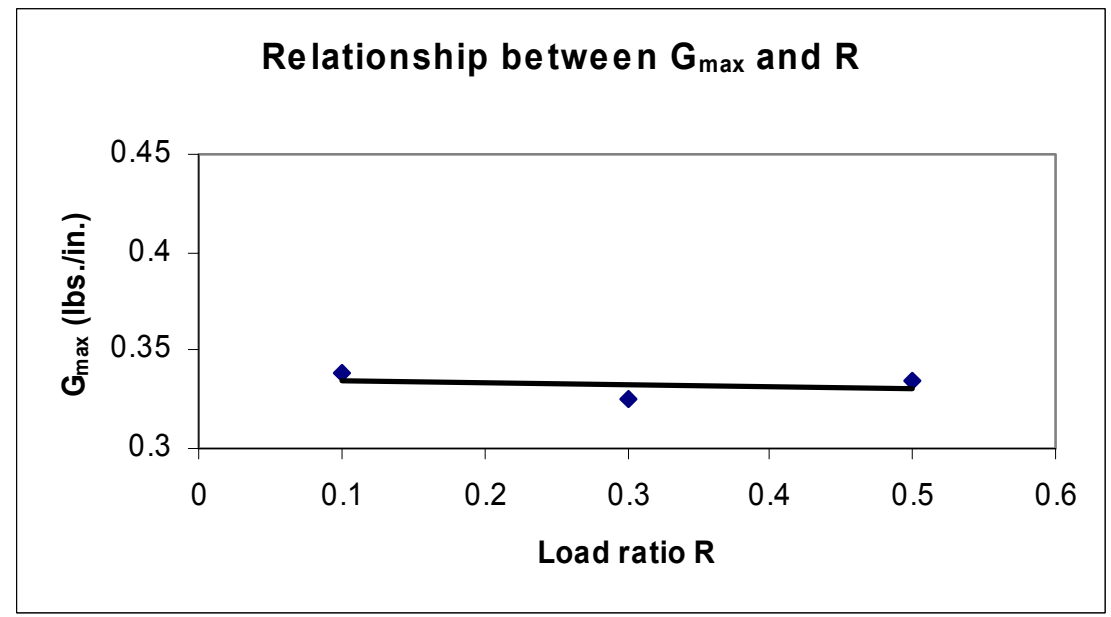

Figure 6.4 Effect of load ratio $R$ on the maximum strain energy release rate $G_{\max }$ at fatigue threshold 


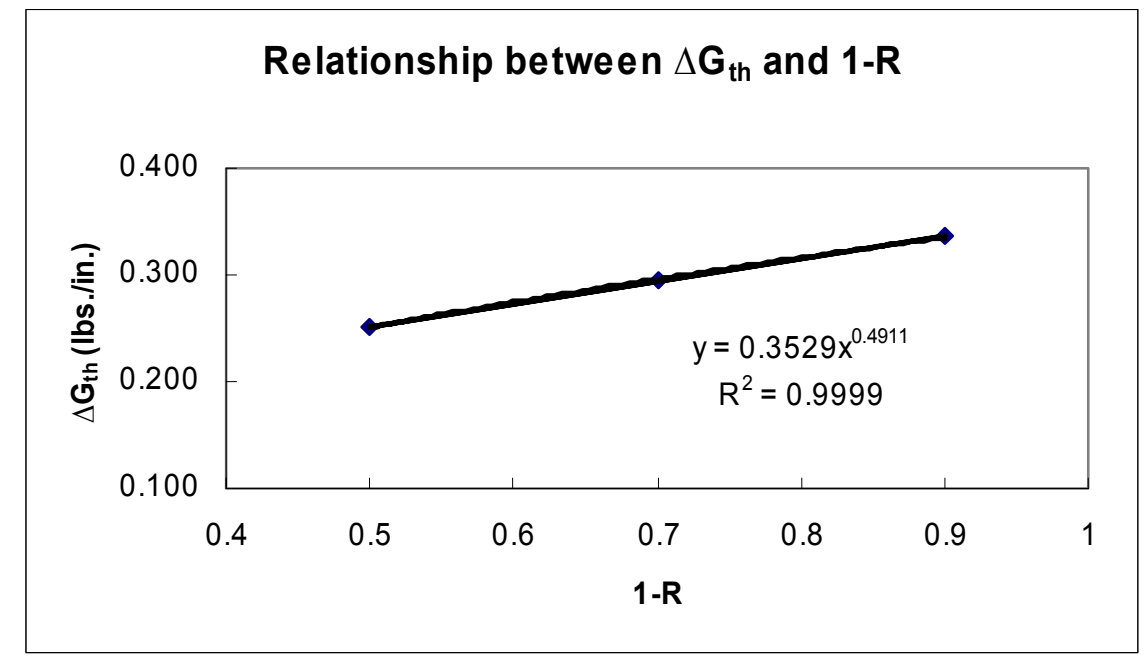

Figure 6.5 The prediction from Klesnil and Lukas's model for the relationship between $\Delta G_{t h}$ and $(1-R)$

\subsection{Conclusions}

In this chapter, the fatigue threshold of the interface bond between red maple wood and phenolic FRP composite is studied. The modified load shedding technique is developed and used to determine the fatigue threshold value of the bonded interface. The load ratio effect on the threshold strain energy release rate range $\Delta G_{t h}$ is investigated. Three sets of tests for load ratios of $0.1,0.3$ and 0.5 are performed at a frequency of $1 \mathrm{~Hz}$ and sinusoidal waveform. All the tests are carried out under constant amplitude cyclic 
loading at room temperature and laboratory conditions. The dependence of the threshold strain energy release rate range, $\Delta G_{t h}$, on load ratio is explained well by the crack closure concept. Also, a phenomenological relationship from Klesnil and Lukas's model (1972) is proposed to explain the load ratio effect on $\Delta G_{t h}$. The results of this chapter are summarized next:

1. It is observed that $G_{\max }$ or $\Delta G /(1-R)$ remains relatively constant at the threshold level over the range of load ratio $R$ tested here.

2. The threshold strain energy release rate range $\Delta G_{t h}$ decreases as the load ratio increases for load ratio $0 \leq R \leq 0.5$.

3. The substantial crack closure effect was observed in this study. This phenomena provided a way to explain the load ratio effect on the fatigue threshold of the bonded interface.

4. Schmidt and Paris's model (1973) can be used to explain the load ratio effect on fatigue threshold of the present bonded interface. The experimental data for load ratio below 0.5 falls into the first regime of the Schmidt and Paris's model.

5. When the load ratio $R$ is less than or equal to 0.5 , the fatigue threshold is $G_{\max }$ controlled, and if the $G_{\max }$ is less than about $0.34 \mathrm{lbs}$./in., the fatigue failure can be ignored for the bonded interface. 
6. The Klesnil and Lukas's model (1972) using strain energy release rate as a controlling parameter can be applied for the purpose of this study:

$$
\Delta G_{t h}=\Delta G_{t h 0}(1-R)^{\gamma}
$$

where $\Delta G_{t h 0}$ is the threshold value for the load ratio $R=0$. The load ratio $R$ sensitivity is governed by a material parameter $\gamma$. In this study, the following values are obtained: $\Delta G_{t h 0}=0.35 \mathrm{lbs} . /$ in. and $\gamma=0.49$. 


\section{CHAPTER 7}

\section{CONCLUSIONS AND RECOMMENDATIONS}

To rehabilitate civil infrastructure, fiber-reinforced polymer (FRP) composites have been used for reinforcement of conventional materials, including wood and concrete. The significant needs for structural retrofitting of facilities in the nation have presented a unique opportunity for the development of FRP materials to be externally bonded to structural members, for both wood and concrete. Current reinforcement of wood members or structures with FRP is focused on externally bonded fabrics or laminates with appropriate adhesives. Significant increases in structural response (stiffness and strength) have been reported by this reinforcement technique; however, there is a concern about the long-term performance of FRP-wood bonded interface, which is susceptible to delamination. The service performance of FRP-wood bonded interface is essential in the design and durability of reinforced wood products. The delamination of the interface bond can lead to premature failure of FRP-wood products.

The performance of FRP-wood bonded interfaces under static and environmental loads has been explored extensively by Davalos et al. (1997, 1998a, 1998b, 2000a, 200b) and Qiao and Davalos (2000). A comprehensive program to evaluate the fatigue behavior 
of phenolic FRP-red maple wood bonded interfaces is presented for the first time in this study. The influence of loading variables, load ratio, waveform and frequency on the crack propagation rate is investigated in separate sections. A modified Paris Law equation is proposed to include both the frequency and load ratio effect. The present study also examines the fatigue threshold behavior of phenolic FRP-red maple wood bonded interfaces. The most significant results are summarized and discussed in terms of practical applications. This study offers new understanding of crack growth behavior of phenolic FRP-red maple wood bonded interfaces.

\subsection{Summary and Conclusions}

A comprehensive study is presented on the fatigue behavior of bonded interfaces for red maple wood bonded to phenolic FRP composite. This study is focused on the possible loading variable (load ratio, waveform and frequency) effect on the crack

propagation rate of the interface, and the behavior of the fatigue threshold is also investigated. The conclusions of this research are given next:

1. The CDCB specimen is shown to be suitable for fatigue tests. The bonded interface displayed the typical crack propagation behavior under constant cyclic loading observed by others.

2. The compliance method used for the CDCB specimen is quite simple, relatively precise and effective for measuring crack propagation rate of interfaces. 
3. The bonded interface under constant cyclic loading exhibits a catastrophic ultimate failure behavior, and the interface bond failure is the primary failure mode.

4. The Paris Law equation using strain energy release rate as a controlling parameter can be efficiently applied to a given value of load ratio $R$ :

$$
\frac{d a}{d N}=B(\Delta G)^{m}
$$

where $B$ and $m$ are material constants; both $B$ and $m$ increase as the load ratio $R$ increases.

5. Any two of the following parameters: $G_{m i n}, G_{m e a n}$, and $R$ combined with $\Delta G$ can characterize crack growth rate under different load ratios. The modified Paris Law equations for the bonded interface are given as

$$
\begin{aligned}
& \frac{d a}{d N}=2 \times 10^{-6}\left(\frac{\Delta G}{(1-R)^{0.79}}\right)^{5.54} \\
& \frac{d a}{d N}=7 \times 10^{-5}\left(\Delta G^{0.87} G_{\text {min }}^{0.13}\right)^{5.34} \\
& \frac{d a}{d N}=1 \times 10^{-4}\left(\Delta G^{0.07} G_{\text {mean }}^{0.93}\right)^{5.59}
\end{aligned}
$$


Any of these proposed equations can efficiently represent the load ratio effect on crack propagation rate of the wood-FRP interfaces, and it can further be used for other similar studies of interface fracture of dissimilar materials.

6. The modified Paris Law equation

$$
\frac{d a}{d N}=1 \times 10^{-4}\left(\Delta G^{0.07} G_{\text {mean }}^{0.93}\right)^{5.59}
$$

can best explain the load ratio effect on the interface fatigue behavior. We strongly recommend this equation for engineering applications.

7. The crack closure effect is observed for the bonded interface, especially for the load ratio of 0.1 . This effect provides a physical explanation of the load ratio effect on the crack propagation rate of the bonded interface.

8. The relationship between effective or equivalent strain energy release rate range ratio $\alpha$ and load ratio $R$ can be expressed as

$$
\alpha=\frac{R}{1-R}+0.5
$$

This gives a good estimation of $\alpha$ as a function of load ratio $R$. 
9. The critical load ratio $R_{\text {critical }}$ corresponding to the effective or equivalent strain energy release rate range ratio $\alpha$ equal to 1 is approximated as 0.33 .

10. $\Delta G$ combined any of the following parameters: $R, G_{\min }, G_{m e a n}$, and $\alpha$ can explain the load ratio effect for the same maximum load.

11. Three waveform shapes considered in this study have little effect, while the load frequency has important effect on crack propagation rate of the interface.

12. The Paris Law equation using strain energy release rate as a controlling parameter can be efficiently applied to a given value of frequency $f$ :

$$
\frac{d a}{d N}=B(\Delta G)^{m}
$$

Both $B$ and $m$ decrease as the frequency $f$ increases.

13. It is believed that the following relationship is valid

$$
\frac{d a}{d N} \propto f^{-\alpha} \Delta G^{0.07} G_{\text {mean }}^{0.93}
$$

where $\alpha$ is a material constant determined empirically. 
14. The following modified Paris Law equation is proposed for the bonded interface

$$
\frac{d a}{d N}=1 \times 10^{-4}\left(f^{-0.05} \Delta G^{0.07} G_{\text {mean }}^{0.93}\right)^{5.66}
$$

This equation can efficiently represent the combined load ratio and frequency effects for crack propagation rate of wood-FRP interface bond, and it can further be used for other similar studies of interface fracture of dissimilar materials.

15. It is observed that $G_{\max }$ or $\Delta G /(1-R)$ remains relatively constant at the threshold level over the range of load ratio $R$ tested here.

16. The threshold strain energy release rate range $\Delta G_{t h}$ decreases as the load ratio increases for load ratio $0 \leq R \leq 0.5$.

17. A substantial crack closure effect was observed in this study. This phenomenon provided a way to explain the load ratio effect on the fatigue threshold of the bonded interface.

18. Schmidt and Paris's model (1973) can be used to explain the load ratio effect on fatigue threshold of the bonded interface. The experimental data for load ratio below 0.5 falls into the first regime of Schmidt and Paris's model. 
19. When the load ratio $R$ is less than or equal to 0.5 , the fatigue threshold is $G_{\max }$ controlled, and if the $G_{\max }$ is less than about $0.34 \mathrm{lbs}$./in., the fatigue failure can be ignored for the bonded interface.

20. The Klesnil and Lukas's model (1972) using strain energy release rate as a controlling parameter can be applied as a comparative study for fatigue threshold:

$$
\Delta G_{t h}=\Delta G_{t h 0}(1-R)^{\gamma}
$$

where $\Delta G_{t h 0}$ is the threshold value for the load ratio $R=0$. The load ratio $R$ sensitivity is governed by a material parameter $\gamma$. In this study, following parameters apply: $\Delta G_{t h 0}=0.35 \mathrm{lbs} . / \mathrm{in}$. and $\gamma=0.49$.

\subsection{Recommendations for Future Work}

Although the research presented here has improved the current understanding of the fatigue behavior of phenolic FRP-red maple wood bonded interfaces, more work is needed. The current research was described in the context of a comprehensive Paris Model, including both load ratio and frequency effects. Ultimately, other influences such as environmental exposure should be included. Future research topics presented here are divided into two parts: (1) crack closure phenomenon, and (2) environmental effect. 
The concept of fatigue crack closure was a conceptual breakthrough for understanding load ratio effects on crack propagation rate. Elber (1970) proposed that closure occurred because fatigue cracks grow through the plastic strain field generated at the crack tip. Once in the crack wake, plastic deformation partially fills the crack mouth causing premature contact that is crack closure. This closure mechanism has been used to explain the load ratio effect on fatigue crack growth rate. Crack closure also can have a dominant influence on fatigue crack growth rate behavior in the near threshold region at low load ratios. This implies that the conditions in the wake of the crack and prior loading history can have bearing on the current propagation rate. The understanding of the role of the closure process is essential to such phenomena as the crack growth rate behavior and the threshold during cyclic loading. Closure provides a mechanism whereby the cyclic fracture toughness near the crack tip differs from the nominally applied values. This concept is of importance to the fracture mechanics interpretation of fatigue crack growth rate data since it implies non-unique growth rate dependence in terms of fracture toughness and load ratio. The closure mechanism observed by Elber (1970) was named plasticity induced crack closure as other closure mechanisms were suggested. Adams (1972) introduced a crack closure due to contact of rough crack surfaces, called roughness induced crack closure. This mechanism occurs when misaligned rough crack surfaces contact during unloading. The resulting contact holds back the crack open, and reduces the effective crack tip driving forces.

To understand the load ratio effect on crack propagation rate and fatigue threshold, a crack closure study is recommended: 
1. Develop an experimental method to determine the crack opening and closing load.

2. Understand the reason that caused the crack closure for phenolic FRP-red maple wood bonded interfaces.

3. Separate the extrinsic load ratio effect (crack closure) from intrinsic load ratio effect (crack closure free).

4. Build an analytical or theoretical model to simulate the crack closure effect and intrinsic load ratio effect.

Environments affect not only the crack growth rate in the Paris region, but also the fatigue threshold in Region I. For example, the most damaging component of laboratory air is water vapor. Oxidation of freshly formed crack surfaces in humid air produces free atomic hydrogen that is drawn into crack tip process zone. Hydrogen embrittlement weakens the material ahead of the crack producing accelerated fatigue crack growth. Although the influence of environment is not within the scope of this study, it is a very important issue and the author recommends a further study to generate a better understanding of fatigue behavior of phenolic FRP-red maple wood bonded interfaces. The ASTM D 2559 standard tests are suggested in combination with fatigue tests to simulate the cyclic environmental and mechanical effects. 


\section{Reference}

Anderson, T. L., Fracture Mechanics: Fundamentals and Applications, Second Edition, CRC Press, Boca Raton, New York, 1994.

Arad, S., Radon, J. C. and Culver, L. E., "Fatigue Crack Propagation in Polymethylmethacrylate; the Effect of the Mean Value of Stress Intensity Factor," Journal Mechanical Engineering Science, Vol. 13, No. 2, 1971, pp. 75-81.

Arad, S., Radon, J. C. and Culver, L. E., "Design against Fatigue Failure in Thermoplastics," Engineering Fracture Mechanics, Vol. 4, No. 3, 1972, pp. 511-522.

ASTM E-647: Annual Book of ASTM Standards, Vol. 03.01, 1995, pp. 578-614.

Atodaria, D. R., Putatunda, S. K., Mallick, P. K., "A Fatigue Crack Growth Model for Random Fiber Composites," Journal of Composite Materials, Vol. 31, No. 18, 1997, pp. 1838-1855.

Atodaria, D. R., Putatunda, S. K., Mallick, P.K., "Delamination Growth Behavior of A Fabric Reinforced Laminated Composite under Mode I Fatigue," Journal of Engineering Materials and Technology, Vol. 121, 1999, pp. 381-385.

Barbero, E., Introduction to Composite Materials Design, First Edition, Taylor and Francis, Philadelphia, PA, 1998.

Branco, C. M., Radon, J. C., Culver, L. E., “An Analysis of the Influence of Mean Stress Intensity and Environment on Fatigue Crack Growth in a New High Strength Aluminum Alloy," Journal of Testing and Evaluation, Vol. 3, No. 6, 1975a, pp. 407-413.

Branco, C. M., Radon, J. C., Culver, L. E., "Influence of Mean Stress Intensity on Fatigue Crack Growth in An Aluminum Alloy," Journal Mechanical Engineering Science, Vol. 17, No. 4, 1975b, pp. 199-205.

Chu, H. P., "Effect of Mean Stress Intensity on Fatigue Crack Growth in a 5456-H117 Al-Alloy," Fracture Toughness and Slow-Stable Cracking, ASTM STP 559, American Society for Testing and Materials, Philadelphia, 1974, pp. 245-261.

Cooke, R. J., Irving, P. E. Booth, G. S. and Beevers, C. J., "The Slow Fatigue Crack Growth and Threshold Behavior of A Medium Carbon Alloy Steel in Air and Vacuum," Engineering Fracture Mechanics, Vol. 7, 1975, pp. 69-77.

Davalos, J. F. and Barbero, E. J., "Advanced Composites in Civil Infrastructure, Testimony before the Subcommittee on Science, Technology, and Space; Committee on Commerce, Science, and Transportation," Hearing on the uses of Advanced Materials in Civil Infrastructure, U.S. Senate, Washington, D. C., May 1993, pp. 35-40. 
Davalos, J. F. and Jia, J., "Fatigue Behavior of FRP-Wood Bonded Interface," Proceedings of CDCC 2002, $2^{\text {nd }}$ International Conference on Durability of FRP Composite for Construction, University of Sherbrooke, Sherbrooke, Quebec, Canada, May 2002.

Davalos, J. F., Madabhusi-Raman, P., and Qiao, P., "Characterization of Mode-I Fracture of Hybrid Material Interface Bonds by Contoured DCB Specimens," Engineering Fracture Mechanics, Vol. 58, No. 3, 1997, pp. 173-192.

Davalos, J. F., Madabhusi-Raman, P., Qiao, P., and Lang, E. M., "Mode-I Fracture Toughness of Fiber Reinforced Composite-Wood Bonded Interfaces," J. of Composite Materials, Vol. 32, No. 10, 1998a, pp. 987-1013.

Davalos, J. F., Madabhusi-Raman, P., Qiao, P., and Wolcott, M. P., "Compliance Rate Change of Tapered Double Cantilever Beam Specimen with Hybrid Interface Bonds," Theoretical and Applied Fracture Mechanics, Vol. 29, No. 2, 1998b, pp. 125-139.

Davalos, J. F. and Qiao, P., "Interface Durability of Construction Materials Externally Reinforced with FRP Composites," in Long Term Durability of Structural Materials, 2001 Elsevier. Monteiro, Chong, Larsen-Base and Komvopoulos (Eds.). Proceedings of the NSF Durability Workshop, Berkeley, CA, Oct. 26-27, 2000, pp. 57-68.

Davalos, J. F., Qiao, P., and Trimble, B. S., "Fiber-Reinforced Composite and Wood Bonded Interface, Part 1. Durability and Shear Strength," ASTM Journal of Composites Technology and Research, Vol. 22, No. 4, 2000a, pp. 224-231.

Davalos, J. F., Qiao, P., and Trimble, B. S., "Fiber-Reinforced Composite and Wood Bonded Interface, Part 2. Fracture," ASTM Journal of Composites Technology and Research, Vol. 22, No. 4, 2000b, pp. 232-240.

Davalos, J. F., Salim, H. A., Qiao, P., Lopez-Anido, R., and Barbero, E. J., “Analysis and Design of Pultruded FRP Shapes under Bending," Composites, Part B, Engineering Journal, Vol. 27, No. 3-4, 1996, pp. 295-305.

Davalos, J. F., Zipfel, M. G., and Qiao, P. Z., "Feasibility study of Prototype GFRPReinforced Wood Railroad Crosstie," ASCE Journal of Composites for Construction, Vol. 3, No. 2, 1999, pp. 92-99.

Dowling, N. E. and Begley, J. A., "Fatigue Crack Growth during Gross Plasticity and the J Integral," Fracture Toughness and Slow-Stable Cracking, ASTM STP 590, American Society for Testing and Materials, Philadelphia, 1976, pp. 82-103.

Dowling, N. E., and Thangjithan, S., "An Overview and Discussion of Basic Methodology for Fatigue," Fatigue and Fracture Mechanics: $31^{\text {st }}$ Volume, ASTM STP 
1389, G. R. Halford and J. P. Gallagher, Eds., American Society for Testing and Materials, West Conshohocken, PA, 2000, pp. 3-36.

Elber, W., "Fatigue Crack Closure under Cyclic Tension," Engineering Fracture Mechanics, Vol. 2, 1970, pp. 37-45.

Elber, W., "The Significance of Fatigue Crack Closure," Damage Tolerance in Aircraft Structures, ASTM STP 486, American Society for Testing and Materials, 1971, pp. 230242.

El-Senussi, A. K. and Webber, J. P. H., "Critical Strain Energy Release Rate during Delamination of Carbon Fiber Reinforced Plastic Laminates," Composites, Vol. 20, May 1989, pp. 249-256.

Hernandez, R., Davalos, J. F., Sonti, S. S., Kim, Y., and Moody, R. C., "Strength and Stiffness of Reinforced Yellow-Poplar Glued-Laminated Beams," Research Paper FRLRP-554, Madison, WI, U. S. Dept. of Agriculture, Forest Service, Forest Products Laboratory, 1997, $28 \mathrm{pp}$.

Forman, R. G., Kearney, V. E., and Engle, R. H., "Numerical Analysis of Crack Propagation in Cyclic Load Structures," Journal of Basic Engineering, Series D, Transactions, American Society of Mechanical Engineers, Vol. 89, No. 3, 1967, pp. 459464.

Hojo, M., Tanaka, K. and Hayashi, R., "Effect of Stress Ratio on Near-Threshold Propagation of Delamination Fatigue Cracks in Unidirectional CFRP," Composite Science and Technology, Vol. 29, 1987, pp. 273-292.

Ishiyama, S., Eto, M. and Oku, T., "Effect of Stress Ratio on Crack Extension Rate of Fine-Grained Isotropic Nuclear Graphite," Journal of Nuclear Science and Technology, Vol. 24, No. 9, 1987, pp. 719-723.

Jia, J., Artificial Neural Network for Mode-I Fatigue of Interface Bonds for Dissimilar Materials, Doctoral Qualifying Examination, West Virginia University, Morgantown, WV, 2002.

Kachanov, L. M., Introduction to Continuum Damage Mechanics, Second Edition, Kluwer Academic Publishers, 1990.

Kim, Y., Davalos, J. F., and Barbero, E. J., "Delamination Buckling of FRP Layer in Laminated Wood Beams," Composite Structures, Vol. 37, No. 3-4, 1997, pp. 311-320.

Klesnil, M. and Lukas, P., "Influence of Strength and Stress History on Growth and Stabilization of Fatigue Cracks," Engineering Fracture Mechanics, Vol. 4, 1972a, pp. 7792. 
Klesnil, M. and Lukas, P., "Effect of Stress Asymmetry on Fatigue Crack Growth," Material Science and Engineering, Vol. 9, 1972b, pp. 231-240.

Liu-Nash, G., Todd, J. A. and Mostovoy, S. "An Accelerated Test Method for Determination Near-Threshold Stress Intensity Values in HSLA Steels," Fatigue Fract. Engng. Mater. Struct. Vol. 20, No. 12, 1997, pp. 1657-1664.

Liu, H. W., Proceedings of the $10^{\text {th }}$ Sagamore Army Materials Research Conference, Syracuse University Press, New York, 1964, pp. 127.

Madabhusi-Raman, P. Analysis of Mode-I Fracture of Wood/Fiber-Reinforced Plastic (FRP) Interface Bond, Master Thesis, West Virginia University, Morgantown, WV, 1995.

Mandell, J. F. and Meier, U., "Fatigue Crack Propagation in $0^{0} / 90^{0}$ E-Glass/Epoxy Composites," Fatigue of Composite Materials, ASTM STP 569, American Society for Testing and Materials, Philadelphia, 1975, pp. 28-44.

Mandell, J. F. and Meier, U., "Effects of Stress Ratio, Frequency, and Loading Time on the Tensile Fatigue of Glass-Reinforced Epoxy," Long-Term Behavior of Composites, ASTM STP 813, O'Brien, T. K., Ed., American Society for Testing and Materials, Philadelphia, 1983, pp. 55-77.

Marci, G., "A Fatigue Crack Growth Threshold," Engineering Fracture Mechanics, Vol. 41, No. 3, 1992, pp. 367-385.

Marcus, H. L. and Sih, G. C. "A Crack-Line-Loaded Edge-Crack Stress Corrosion Specimen," Engineering Fracture Mechanics, Vol. 3, 1971, pp. 453-461.

Mendenhall, W., Beaver, J. R. and Beaver, M. B., Introduction to Probability and Statistics, Tenth Edition, Duxbury Press, 1999.

Mizutani, K. and Iwatsu, T., "Influence of External Environments on Fatigue Crack Growth in Epoxy Resin," Polymer Engineering and Science, Vol. 23, No. 4, Mar. 1983, pp. 183-185.

Moskala, J. Eric, "Effects of Mean Stress and Frequency on Fatigue Crack Propagation in Rubber-Toughened Polycarbonate/Copolyester Blends," Journal of Applied Polymer Science, Vol. 49, 1993, pp. 53-64.

Mostovoy, S., Crosley, P. B. and Ripling, E. J., "Use of Crack-Line-Loaded specimens for Measuring Plain-Strain Fracture Toughness," J. of Materials, Vol. 2, No. 3, 1967, pp. 661-681.

Mostovoy, S., and Ripling, E. J., "Flaw Tolerance of a Number of Commercial and Experimental Adhesives," Polymer Science Technology B, Vol. 9B, 1975, pp. 513-562. 
Nakai, Y., Tanaka, K. and Nakanishi, T., "The Effects of Stress Ratio and Grain Size on Near-Threshold Fatigue Crack Propagation in Low-Carbon Steel," Engineering Fracture Mechanics, Vol. 15, No. 3-4, 1981, pp. 291-302.

Ohta, A. and Sasaki, E., "Influence of Stress Ratio on the Threshold Level for Fatigue Crack propagation in High Strength Steels," Engineering Fracture Mechanics, Vol. 9, 1977, pp. 307-315.

Paris, P. C., Gomez, M. P. and Anderson, W. E., "Rational Analytical Theory of Fatigue," The Trend in Engineering, Vol. 13, No. 1, 1961, pp. 9-14.

Paris, P. C. and Erdogan, F., "Critical Analysis of Propagation Laws," J. of Basic Engineering, Vol. 85, 1963, pp. 528-534.

Pourartip, A., Ashby, M. F. and Beaumont, P.W. R., "The Fatigue Damage Mechanics of A Carbon Fiber Composite Laminate: I---Development of the Model," Composites Science and Technology, Vol. 25, 1986, pp. 193-218.

Pourartip, A. and Beaumont, P.W. R., "The Fatigue Damage Mechanics of A Carbon Fiber Composite Laminate: II---Life Prediction," Composites Science and Technology, Vol. 25, 1986, pp. 283-299.

Qiao, P. Z., Davalos, J. F. and Trimble, B. S., "Effect of Moisture on Fracture Toughness of Composite/Wood Bonded Interfaces," Fatigue and Fracture Mechanics: $31^{\text {st }}$ Volume, ASTM STP 1389, G. R. Halford and J. P. Gallagher, Eds., American Society for Testing and Materials, West Conshohocken, PA, 2000, pp. 526-544.

Qiao, P. Z., Davalos, J. F., and Zipfel, M. G., "Modeling and Optimal Design of Composite-Reinforced Wood Railroad Crosstie," Composite Structures, Vol. 41, No. 1, 1998, pp. 87-96.

Qiao, P. Z., Wang, J. and Davalos, J. F., "Mode-II Fracture of Wood-Composite Bonded Interfaces using Tapered ENF Specimens," Proceedings of the $34^{\text {th }}$ International SAMPE Technical Conference - 2002 M\&P - Ideas to Reality. Vol. 34, Baltimore, MD, 2002, Society for the Advancement of Material and Process Engineering, pp. 872-884.

Radon, J. C., Arad, S and Culver, L. E., "Growth of Fatigue Cracks in Metals and Polymers," Engineering Fracture Mechanics, Vol. 6, 1974, pp. 195-208.

Ritchie, R. O., Boyce, B. L., Campbell, J. P., Roder, O., Thompson, A. W. and Miligan, W. W., "Thresholds for High-Cycle Fatigue in a Turbine Engine Ti-6A1-4V alloy," International Journal of Fatigue, Vol. 21, 1999, pp. 653-662.

Russell, A. J. and Street, K. N., "A Constant $\Delta \mathrm{G}$ Test for Measuring Mode I Interlaminar Fatigue Crack Growth Rates," Composite Materials: Testing and Design (Eighth 
Conference), ASTM STP 972, Whitcomb, J. D. Ed., American Society for testing and Materials, Philadelphia, 1988, pp. 259-277.

Schmidt, R. A. and Paris, P. C., "Threshold for Fatigue Crack Propagation and the Effects of Load Ratio and Frequency," Progress in Flaw Growth and Fracture Toughness Testing, ASTM STP 536, American Society for Testing and Materials, 1973, pp. 79-94.

Singh, N., Khelawan, N. and Mathur, G N, "Effect of Stress Ratio and Frequency on Fatigue Crack Growth Rate of 2618 Aluminum Alloy Silicon Carbide Metal Matrix Composite," Bulletin of Materials Science, Vol. 24, No. 2, 2001, pp. 169-171.

Solomon, H. D, "Frequency Dependent Low-Cycle Fatigue Crack Propagation," Metal. Trans., Vol. 4, 1973, pp. 341-347.

Sun, C. T. and Chan, W. S., "Frequency Effect on the Fatigue Life of a Laminated Composite," Composite Materials: Testing and Design (Fifth Conference), ASTM STP 674, S. W. Tsai, Ed., American Society for Testing and Materials, 1979, pp. 418-430.

Suresh, S., Fatigue of Materials, Second Edition, Cambridge University Press, Cambridge, UK, 1998.

Suresh, S., and Ritchie, R. O., "Near-Threshold Fatigue Crack Propagation: A Perspective on the Role of Crack Closure," Fatigue Crack Growth Threshold Concepts, TMS-AIME, Davidson, D. L., Suresh, S., editors, Warrendale, PA, 1984, pp. 227-261.

Sutton, A. S, "Fatigue Crack Propagation in an Epoxy Polymer," Engineering Fracture Mechanics, Vol. 6, 1974, pp. 587-595.

Tanaka, K., "Mechanics and Micromechanics of Fatigue Crack Propagation," Fracture Mechanics: Perspectives and Directions (Twentieth Symposium), ASTM STP 1020, R. P. Wei and R. P. Gangloff, editors, American Society for Testing and Materials, Philadelphia, 1989, pp. 151-183.

Tong, J. and Byrne, J., "Effects of Frequency on Fatigue Crack Growth Rate at Elevated Temperature," Fatigue Fracture Engineering Material Structure, Vol. 22, 1999, pp. 185193.

Trimble, B. S., Durability and Mode-I Fracture of Fiber-Reinforced Plastic (FRP)/Wood Interface Bond, Master Thesis, West Virginia University, Morgantown, WV, 1999.

Tsuji, H., Nakajima, H., and Kondo, T., "Effect of $\Delta \mathrm{K}$ Control Modes on Variability/Reproducibility of Fatigue Crack Growth Rate Data," Journal of Nuclear Materials, Vol., 189, 1992, pp. 65-71. 
Walker, K. "The Effect of Stress Ratio During Crack Propagation and Fatigue for 2024T3 and 7075-T6 Aluminum," Effects of Environment and Complex Load History on Fatigue Life, ASTM STP 462, American Society for Testing and Materials, 1970, pp. 114.

Weertman, J., "Rate of Growth of Fatigue Cracks Calculated from the Theory of Infinitesimal Dislocations Distributed on a Plane," International Journal of fracture Mechanics, Vol. 2, 1966, pp. 460-467.

Williams, J. G., "A Model of Fatigue Crack Growth in Polymers," Journal of Materials Science, Vol. 12, 1977, pp. 2525-2533. 